\title{
Unitary representations of real reductive groups
}

\author{
Jeffrey D. Adams* \\ Department of Mathematics \\ University of Maryland \\ Marc van Leeuwen \\ Laboratoire de Mathématiques et Applications \\ Université de Poitiers \\ Peter E. Trapa ${ }^{\dagger}$ \\ Department of Mathematics \\ University of Utah \\ David A. Vogan, Jr. ${ }^{\ddagger}$ \\ Department of Mathematics \\ Massachusetts Institute of Technology
}

January 22, 2013

\begin{abstract}
We present an algorithm for computing the irreducible unitary representations of a real reductive group $G$. The Langlands classification, as formulated by Knapp and Zuckerman, exhibits any representation with an invariant Hermitian form as a deformation of a unitary representation from Harish-Chandra's Plancherel formula. The behavior of these deformations was approximately determined in the KazhdanLusztig analysis of irreducible characters; more complete information comes from the Beilinson-Bernstein proof of the Jantzen conjectures.

Our algorithm follows the signature of the form through this deformation, counting changes at reducibility points. An important tool is Weyl's "unitary trick:" replacing the classical invariant Hermitian form (where $\operatorname{Lie}(G)$ acts by skew-adjoint operators) by a new one where a compact form of $\operatorname{Lie}(G)$ acts by skew-adjoint operators.
\end{abstract}

${ }^{*}$ All of the authors were supported in part by NSF grant DMS-0968275. The first author was supported in part by NSF grant DMS-0967566.

${ }^{\dagger}$ The third author was supported in part by NSF grant DMS-0968060.

¥The fourth author was supported in part by NSF grant DMS-0967272. 


\section{Contents}

1 First introduction $\quad 6$

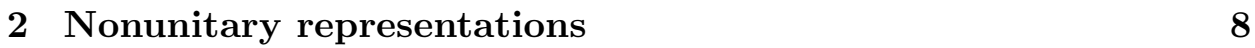

3 Real reductive groups 18

4 Maximal tori $\quad 22$

5 Coverings of tori $\quad 26$

6 Langlands classification and the nonunitary dual 39

7 Second introduction: the shape of the unitary dual 46

8 Hermitian forms on $(\mathfrak{h}, L(\mathbb{C}))$-modules $\quad 48$

9 Interlude: realizing standard modules $\quad 63$

10 Invariant forms on irreducible representations $\quad 71$

11 Standard and $c$-invariant forms in the equal rank case $\quad 81$

12 Twisting by $\theta \quad 82$

13 Langlands parameters for extended groups $\quad 89$

14 Jantzen filtrations and Hermitian forms 106

15 Signature characters for $c$-invariant forms $\quad 119$

16 Translation functors: first facts 131

17 Translation functors for extended groups 144

$18 \mathrm{KLV}$ theory $\quad 159$

$19 \mathrm{KLV}$ theory for extended groups $\quad 169$

$20 \mathrm{KLV}$ theory for $c$-invariant forms $\quad 174$

21 Deformation to $\nu=0 \quad 184$ 


\section{Index of notation}

\begin{tabular}{|c|c|}
\hline $\begin{array}{l}(A, \nu) \\
{ }^{\delta} G(\mathbb{C}),{ }^{\delta} G,{ }^{\delta} K\end{array}$ & $\begin{array}{l}\text { continuous parameter; Definition } 6.5 \text {. } \\
\text { extended groups; Definition 12.3. }\end{array}$ \\
\hline$\widehat{G}_{u}$ & unitary dual of $G ;(1.1 \mathrm{~d})$. \\
\hline$\widehat{G}$ & nonunitary dual of $G$; Definition 2.14 . \\
\hline$G(\mathbb{R}, \sigma)$ & $\begin{array}{l}\text { real points of a real form of a complex Lie group } \\
G(\mathbb{C}) ;(3.1 \mathrm{e}) \text {. }\end{array}$ \\
\hline $\mathcal{G}(\mathfrak{h}, L(\mathbb{C}))^{\sigma}$ & $\begin{array}{l}\text { Grothendieck group of finite length admissible } \\
(\mathfrak{h}, L(\mathbb{C})) \text { modules with nondegenerate } \sigma \text {-invariant } \\
\text { Hermitian forms: Definition } 15.6 \text {. }\end{array}$ \\
\hline$\Gamma=\left(H, \gamma, R_{i \mathbb{R}}^{+}\right)$ & $\begin{array}{l}\text { (continued) Langlands parameter; Definitions 6.2, } \\
6.3 \text {. }\end{array}$ \\
\hline$\Gamma_{1}=\left({ }^{1} H, \gamma, R_{i \mathbb{R}}^{+}\right)$ & extended Langlands parameter; Definition 13.7. \\
\hline & $\begin{array}{l}\text { Hermitian dual (or } c \text {-Hermitian dual) of a Langlands } \\
\text { parameter; Definitions } 10.3,10.6 \text {. }\end{array}$ \\
\hline$H \simeq T \times A$ & $\begin{array}{l}\text { Cartan decomposition of a real torus; Proposition } \\
\text { 4.3. }\end{array}$ \\
\hline 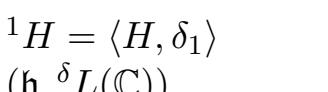 & extended maximal torus; Definition 13.5 . \\
\hline 7, & Cartan involution; (3.4a). \\
\hline$\Theta_{\pi}$ & distribution character of $\pi$; Definition 5.11 . \\
\hline$I_{\mathrm{quo}}(\Gamma), I_{\mathrm{sub}}(\Gamma)$ & $\begin{array}{l}\text { standard quotient-type and sub-type modules; The- } \\
\text { orem 9.2. }\end{array}$ \\
\hline$\ell(\Gamma)$ & integral length of $\Gamma$; Definition 18.2. \\
\hline$\ell_{o}(\Gamma)$ & orientation number of $\Gamma$; Definition 20.5. \\
\hline$\Lambda=\left(T, \lambda, R_{i \mathbb{R}}^{+}\right)$ & discrete Langlands parameter; Definition 6.5. \\
\hline$m_{\Xi, \Gamma}, M_{\Gamma, \Psi}$ & $\begin{array}{l}\text { entries of multiplicity matrices for characters of ir- } \\
\text { reducible and standard modules; (15.1). }\end{array}$ \\
\hline$\left(\operatorname{pos}_{V}, \operatorname{neg}_{V}, \operatorname{rad}_{V}\right)$ & $\begin{array}{l}\text { signature character of a } \sigma \text {-invariant Hermitian form } \\
\text { on an admissible }(\mathfrak{h}, L(\mathbb{C})) \text { module } V \text {; Proposition } \\
8.9(5) \text {. }\end{array}$ \\
\hline$P_{\Gamma, \Psi}$ & character polynomial; Definition 18.12 . \\
\hline$P_{\Gamma, \Psi}^{c}$ & signature character polynomial; Definition 20.4 . \\
\hline$Q_{\Gamma, \Psi}$ & multiplicity polynomial; Definition 18.13. \\
\hline$Q_{\Gamma, \Psi}^{c}$ & signature multiplicity polynomial; Definition 20.2 . \\
\hline$R_{\mathbb{R}}, R_{i \mathbb{R}}, R_{\mathbb{C}}$ & $\begin{array}{l}\text { real, imaginary, and complex roots in } R \text {; Definition } \\
5.7 \text {. }\end{array}$ \\
\hline & 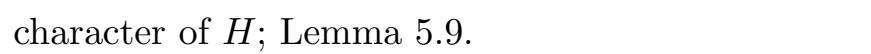 \\
\hline
\end{tabular}




$\begin{array}{ll}\sigma & \text { real form of a complex algebraic variety or complex } \\ & \text { Lie group; }(2.16),(3.1 \mathrm{c}) \text {. } \\ \sigma_{c} & \text { compact real form of a complex connected reductive } \\ & \text { Lie group; Example } 3.2 . \\ \tau(\Gamma) & \tau \text {-invariant of } \Gamma \text {; Definition } 16.7 . \\ t_{1}, t_{s} & \text { extended Weyl group elements for extended tori }{ }^{1} H, \\ & { }^{1} H_{s} ; \text { Definition } 13.5, \text { Proposition } 17.2 . \\ \left(\pi^{h, \sigma}, V^{h, \sigma}\right) & \sigma \text {-Hermitian dual of a }(\mathfrak{h}, L(\mathbb{C})) \text {-module } V ;(8.5) . \\ \mathbb{W} & \text { signature ring; Definition } 15.9 . \\ W^{\Lambda} & \text { stabilizer of } \Lambda \text { in the real Weyl group; Proposition } \\ & 6.6(1) \text {. } \\ W(G, H) & \text { real Weyl group; Definition } 5.7 \text {, Proposition } 5.8(4) . \\ { }^{\prime} W(G, H) & \text { extended real Weyl group; Definition } 13.5 . \\ X(\mathbb{R}, \sigma) & \text { real points of the real form } \sigma \text { of the complex alge- } \\ & \text { braic variety } X(\mathbb{C}) ;(2.16) . \\ X^{*}, X_{*} & \text { character and cocharacter lattices of a maximal } \\ & \text { torus; Definition } 4.1 .\end{array}$




\section{First introduction}

$\{$ sec:firstintro $\}$

The purpose of this paper is to give a finite algorithm for computing the set of irreducible unitary representations of a real reductive Lie group $G$. Even before explaining the nature of the algorithm, it is worth recalling why this is an interesting question. A serious historical survey would go back at least to the work of Fourier (which can be understood in terms of the irreducible unitary representations of the circle).

Since we are not serious historians, we will begin instead with a formulation of "abstract harmonic analysis" arising from the work of Gelfand beginning in the 1930s. In Gelfand's formulation, one begins with a topological group $G$ acting on a topological space $X$. A reasonable example to keep in mind is $G=G L(n, \mathbb{R})$ acting on the space $X$ of lattices in $\mathbb{R}^{n}$. What makes such spaces difficult to study is that there is little scope for using algebra.

The first step in Gelfand's program is therefore to find a nice Hilbert space $\mathcal{H}$ (often of functions on $X$ ); for example, if $G$ preserves a measure on $X$, then one can take

$$
\mathcal{H}=L^{2}(X)
$$

If choices are made well (as in the case of an invariant measure, for example) then $G$ acts on the Hilbert space $\mathcal{H}$ by unitary operators:

$$
\pi: G \rightarrow U(\mathcal{H}) .
$$

Such a group homomorphism (assumed to be continuous, in the sense that the map

$$
G \times \mathcal{H} \rightarrow \mathcal{H}, \quad(g, v) \mapsto \pi(g) v
$$

is continuous) is called a unitary representation of $G$. Gelfand's program says that questions about the action of $G$ on $X$ should be recast as questions about the unitary representation of $G$ on $\mathcal{H}$, where one can bring to bear tools of linear algebra.

One of the most powerful tools of linear algebra is the theory of eigenvalues and eigenvectors, which allow some problems about linear transformations to be reduced to the case of dimension one, and the arithmetic of complex numbers. An eigenvector of a linear transformation is a one-dimensional subspace preserved by the linear transformation. In unitary representation theory the analogue of an eigenvector is an irreducible unitary representation: a nonzero unitary representation having no proper closed subspaces invariant under $\pi(G)$. Just as a finite-dimensional complex vector space is a direct sum of eigenspaces of any (nice enough) linear transformation, so 
any (nice enough) unitary representation is something like a direct sum of irreducible unitary representations.

The assumption that we are looking at a unitary representation avoids the difficulties (like nilpotent matrices) attached to eigenvalue decompositions in the finite-dimensional case; but allowing infinite-dimensional Hilbert spaces introduces complications of other kinds. First, one must allow not only direct sums but also "direct integrals" of irreducible representations. This complication appears already in the case of the action of $\mathbb{R}$ on $L^{2}(\mathbb{R})$ by translation. The decomposition into one-dimensional irreducible representations is accomplished by the Fourier transform, and so involves integrals rather than sums.

For general groups there are more serious difficulties, described by von Neumann's theory of "types." But one of Harish-Chandra's fundamental theorems ([9], Theorem 7) is that real reductive Lie groups are "type I," and therefore that any unitary representation of a reductive group may be written uniquely as a direct integral of irreducible unitary representations. The second step in Gelfand's program is to recast questions about the (reducible) unitary representation $\pi$ into questions about the irreducible representations into which it is decomposed.

The third step in Gelfand's program is to describe all of the irreducible unitary representations of $G$. This is the problem of "finding the unitary dual"

$$
\widehat{G}_{u}={ }_{\text {def }}\{\text { equiv. classes of irr. unitary representations of } G\}
$$

It is this problem for which we offer a solution (for real reductive $G$ ) in this paper. It is far from a completely satisfactory solution for Gelfand's program; for of course what Gelfand's program asks is that one should be able to answer interesting questions about all irreducible unitary representations. (Then these answers can be assembled into answers to the questions about the reducible representation $\pi$, and finally translated into answers to the original questions about the topological space $X$ on which $G$ acts.) We offer not a list of unitary representations but a method to calculate the list. To answer general questions about unitary representations in this way, one would need to study how the questions interact with our algorithm.

Which is to say that we may continue to write papers after this one.

We will offer a more complete description of the nature of the algorithm in Section 7, after recalling the results of Harish-Chandra, Langlands, and others in terms of which the algorithm is formulated. For the moment we can say that the algorithm calculates not only the set of unitary representations, 
but more generally the signature of the invariant Hermitian form on any irreducible representation admitting one. What the algorithm uses is the Kazhdan-Lusztig polynomials describing irreducible characters of $G$, and their interpretation (established by Beilinson and Bernstein in [3]) in terms of Jantzen filtrations. The algorithm in the analogous setting of highest weight modules is treated by Yee in $[45,46]$.

We are deeply grateful to George Lusztig for producing the mathematics in [27], without which we would have been unable to complete this work. We thank Annegret Paul, whose expert reading of a draft of this paper led to many emendations and improvements. Finally, we thank Wai Ling Yee, whose work first showed us that an analysis of unitary representations along these lines might be possible; indeed we hoped to persuade her to be a coauthor.

\section{Nonunitary representations}

In this section we will recall Harish-Chandra's framework for the study of representation theory for real reductive groups. Gelfand's program says that unitary representations are what one should care about most, partly because of their value in applications. It is also true that working with infinite-dimensional nonunitary representations leads to terrible difficulties from functional analysis. The difficulties are related to those encountered in studying eigenvalues for linear operators: for normal operators (those commuting with their adjoints, and therefore nicely related to the Hilbert space structure) there is a clean and complete theory, but for general operators everything goes wrong.

On the other hand, it turns out to be extremely difficult to understand unitary representations without also looking at some nonunitary representations. What happens in the end is that we look at certain families of possibly nonunitary representations that are parametrized by complex vector spaces (naturally defined over the rational numbers $\mathbb{Q}$ ). The unitary representations are parametrized by real points of rational polyhedra inside these complex vector spaces. This fact essentially goes back to work of Knapp and Stein in the 1960s, and is made precise in Chapter 16 of [19]. What our algorithm does is compute these polyhedra explicitly. The point is that we are therefore describing unitary representations as a subset of a (much simpler) set of possibly nonunitary representations.

The first big idea is therefore Harish-Chandra's classical one, providing a category of possibly nonunitary representations for which functional analysis 
works nearly as well as in the unitary case. In order to formulate HarishChandra's definition of this category, we need some general terminology about groups and representations.

Suppose $H$ is a real Lie group. The real Lie algebra of $H$ and its com$\{$ se:reps $\}$ plexification are written

$$
\mathfrak{h}_{0}=\operatorname{Lie}(H), \quad \mathfrak{h}=\mathfrak{h}_{0} \otimes_{\mathbb{R}} \mathbb{C} .
$$

The universal enveloping algebra is written $U(\mathfrak{h})$. The group $H$ acts on $\mathfrak{h}$ and on $U(\mathfrak{h})$ by automorphisms

$$
\operatorname{Ad}: H \rightarrow \operatorname{Aut}(U(\mathfrak{h}))
$$

we write

$$
\mathfrak{Z}(\mathfrak{h})=U(\mathfrak{h})^{\operatorname{Ad}(H)}
$$

for the algebra of invariants. If $H$ is connected, or if $H$ is the group of real points of a connected complex group, then $\mathfrak{Z}(\mathfrak{h})$ is precisely the center of $U(\mathfrak{h})$, but in general we can say only

$$
\mathfrak{Z}(\mathfrak{h}) \subset \text { center of } U(\mathfrak{h}) \text {. }
$$

A continuous representation of $H$ is a complete locally convex topological vector space $V$ equipped with a continuous action of $H$ by linear transformations. We will sometimes write representations with module notation, and sometimes with a named homomorphism

$$
\pi: H \rightarrow \operatorname{Aut}(V) .
$$

In either case the continuity assumption means that the map

$$
H \times V \rightarrow V, \quad(h, v) \mapsto h \cdot v=\pi(h) v
$$

is continuous. We mention again that the representation is called unitary if $V$ is a Hilbert space, and the action of $H$ preserves the inner product.

An invariant subspace is a closed subspace $W \subset V$ preserved by the action of $H$. We say that $V$ is irreducible if it has exactly two invariant subspaces (namely 0 and $V$, which are required to be distinct).

The space of smooth vectors in $V$ is

$$
V^{\infty}=\{v \in V \mid \text { the map } h \mapsto h \cdot v \text { from } H \text { to } V \text { is smooth }\} \text {. }
$$

$\{$ eq: smooth $\}$

There is a natural complete locally convex topology on $V^{\infty}$, and the inclusion in $V$ is continuous with dense image. The space $V^{\infty}$ is preserved by 
the action of $H$, in this way becoming a continuous representation in its own right. It carries at the same time a natural (continuous) real Lie algebra representation of $\mathfrak{h}_{0}$ (equivalently, a complex algebra action of $U(\mathfrak{h})$ ) obtained by differentiating the action of $H$. These two representations are related by

$$
h \cdot(X \cdot v)=(\operatorname{Ad}(h) X) \cdot(h \cdot v) \quad\left(h \in H, X \in \mathfrak{h}_{0}, v \in V^{\infty}\right) ;
$$

or in terms of the enveloping algebra

$$
h \cdot(u \cdot v)=(\operatorname{Ad}(h) u) \cdot(h \cdot v) \quad\left(h \in H, u \in U(\mathfrak{h}), v \in V^{\infty}\right) .
$$

If $\left(\pi_{1}, V_{1}\right)$ and $\left(\pi_{2}, V_{2}\right)$ are two representations of $H$, an intertwining operator is a continuous linear map respecting the two actions of $H$ :

$$
T: V_{1} \rightarrow V_{2}, \quad T \pi_{1}(h)=\pi_{2}(h) T \quad(h \in H) .
$$

The vector space of all intertwining operators from $V_{1}$ to $V_{2}$ is written $\operatorname{Hom}_{H}\left(V_{1}, V_{2}\right)$.

Intertwining operators are the morphisms in the category of representations, and therefore they play a central part. Of particular interest are the self-intertwining operators $T \in \operatorname{Hom}_{H}(V, V)$; for any eigenspace of such an operator $T$ is automatically an invariant subspace of $V$. Here is an application.

Proposition 2.2. Suppose $(\pi, V)$ is a continuous representation of a Lie group $H$. If the corresponding smooth representation $\left(\pi^{\infty}, V^{\infty}\right)$ (see (2.1f)) is irreducible, then $\pi$ is irreducible as well.

For any $z \in \mathfrak{Z}(\mathfrak{h})$ (see (2.1c)), the operator $\pi^{\infty}(z)$ is an intertwining operator for $\pi^{\infty}$. If any $\pi^{\infty}(z)$ has any nonzero eigenvector, then either $\pi^{\infty}$ is reducible, or $\pi^{\infty}(z)$ is a scalar operator.

This proposition says that if $\pi$ is an irreducible representation, and $z \in \mathfrak{Z}$, then $\pi^{\infty}(z)$ "ought to be" a scalar operator; that if it is not, then there is some kind of pathological behavior going on, like an operator having no spectral decomposition at all. Such pathological behavior can certainly happen, even in the representation theory of semisimple Lie groups. There is an example due to Soergel in [35]. Harish-Chandra's idea was to use the absence of this pathology to define a reasonable category of nonunitary representations. Here is his definition. (The definition of "reductive group" is of course logically even more fundamental than that of "irreducible representation;" but it is less important for understanding the results, and so we postpone it to Section 3.) 
Definition 2.3. (Harish-Chandra [9], page 225). Suppose $G$ is a real $\{$ def :quasisimple $\}$ reductive Lie group. A continuous representation $(\pi, V)$ of $G$ is called quasisimple if every $z \in \mathfrak{Z}(\mathfrak{g})((2.1 \mathrm{c}))$ acts by scalars on $V^{\infty}$. In this case the algebra homomorphism

$$
\chi_{\pi}: \mathfrak{Z} \rightarrow \mathbb{C}, \quad \pi(z)=\chi_{\pi}(z) \operatorname{Id}_{V^{\infty}}
$$

is called the infinitesimal character of $\pi$.

Harish-Chandra's "good" nonunitary representations are the irreducible quasisimple representations. In order to state his basic results about these representations, and about how the unitary representations appear among them, we need one more bit of technology. The difficulty is that these nonunitary representations do not yet form a nice category: there are not enough intertwining operators.

Here is an illustrative example. The group $G=S L(2, \mathbb{R})$ acts by linear transformations on $\mathbb{R}^{2}$, and therefore on the real projective space $\mathbb{R P}^{1}$ of lines in $\mathbb{R}^{2}$; this is just a circle. On the circle (as on any manifold) we can define a complex line bundle $\mathcal{L}_{1 / 2}$ of half densities; a smooth section of $\mathcal{L}_{1 / 2}$ may be written as $f(\theta)|d \theta|^{1 / 2}$, with $f$ a smooth $2 \pi$-periodic function. There is a natural pre-Hilbert space structure on these sections, with

$$
\left\langle f_{1}|d \theta|^{1 / 2}, f_{2}|d \theta|^{1 / 2}\right\rangle=\int_{0}^{2 \pi} f_{1}(\theta) \overline{f_{2}(\theta)} d \theta
$$

The full group of diffeomorphisms of the circle acts on the space $C^{\infty}\left(\mathcal{L}^{1 / 2}\right)$; the factor $|d \theta|^{1 / 2}$ transforms by the square root of the absolute value of the Jacobian. Consequently this action is by unitary operators, and it extends to the Hilbert space completion $L^{2}\left(\mathcal{L}^{1 / 2}\right)$. We can restrict this representation to $S L(2, \mathbb{R})$, obtaining a unitary representation $\pi^{(2)}$. This turns out to be an irreducible unitary representation; it is called the "spherical principal series with parameter zero." (The word "spherical" refers to the fact that there is a vector fixed by the rotation subgroup $S O(2)$, namely the standard half-density $1 \cdot|d \theta|^{1 / 2}$.)

The difficulty arises because there are many variants of this representation. We can for example consider the representation $\pi^{\infty}$ on the original space $C^{\infty}\left(\mathcal{L}^{1 / 2}\right)$; with the standard locally convex topology on $C^{\infty}$, this is again an irreducible representation. Or we could require the function $f(\theta)$ to be in $L^{p}$ rather than $L^{2}$; although the $L^{p}$ norm is not preserved by diffeomorphisms, they still act by bounded operators, and define an irreducible 
representation $\pi^{(p)}$ for $1 \leq p<\infty$. (The action $\pi^{(\infty)}$ on bounded functions is not continuous in the $L^{\infty}$ norm.) There is an irreducible representation $\pi^{-\infty}$ on distribution sections, and one $\pi^{\omega}$ on analytic sections. There are Sobolev spaces, and functions with a finite number of continuous derivatives. In order to get a nice classification of representations, one must regard all of these representations as essentially the same; but the questions of what intertwining operators exist among the spaces is complicated. (For instance, there is a continuous inclusion of $L^{p}$ in $L^{q}$ whenever $p>q$, since the circle has finite measure, and therefore an intertwining operator from $\pi^{(p)}$ to $\pi^{(q)}$; but there is no nonzero intertwining operator from $\pi^{(q)}$ to $\pi^{(p)}$.)

In the case of the circle, all of these function spaces contain the trigonometric polynomials, and of course the theory of Fourier series offers good descriptions of many (not all!) of them in terms of growth conditions on the Fourier coefficients. The technical difficulty for doing representation theory in this way is that the group $S L(2, \mathbb{R})$ does not preserve the space of trigonometric polynomials. Harish-Chandra observed that the action of the universal enveloping algebra does preserve trigonometric polynomials, and that this property can be generalized to all reductive groups. He showed that some part of the group action is determined by differential equations involving only these trigonometric polynomials, even though the solutions of the equations may have infinite Fourier series.

Part of Harish-Chandra's definition makes sense in a very general setting. Assume again that $H$ is a real Lie group, and fix an arbitrary choice of

$$
L=\text { compact subgroup of } H \text {. }
$$

If $(\pi, V)$ is any representation of $H$, we define

$$
V_{L}=\{v \in V \mid \operatorname{dim}(\langle\pi(L) v\rangle)<\infty\}
$$

here $\langle\pi(L) v\rangle$ denotes the span of the vectors $\pi(l) v$ as $l$ runs over $L$. This is the smallest $L$-invariant subspace of $V$ containing $v$, so we call $V_{L}$ the space of $L$-finite vectors of $\pi$. Let us write

$$
\widehat{L}=\left\{\begin{array}{l}
\text { equivalence classes of irreducible } \\
\text { representations of } L
\end{array}\right\}
$$

Using standard facts about finite-dimensional representations of compact groups, one finds that

$$
V_{L}=\sum_{\delta \in \widehat{L}} V(\delta),
$$


with

$$
V(\delta)=\text { largest sum of copies of } \delta \text { in } V,
$$

the space of $\delta$-isotypic vectors in $V$. There is a natural complete locally convex topology on $V_{L}$, making it the algebraic direct sum of the subspaces $V(\delta)$; each $V(\delta)$ is a closed subspace of $V$.

Lemma 2.9 (cf. [9]). Suppose $V$ is a representation of a real Lie group $H$, and $L$ is a compact subgroup of $H$. Then the space $V_{L}^{\infty}$ of smooth $L$-finite vectors ((2.1f), (2.6)) is preserved by the actions of $L$ and of $\mathfrak{h}_{0}$ (equivalently, of $U(\mathfrak{h}))$. These two actions satisfy the conditions

1. the representation of $L$ is a direct sum of finite-dimensional irreducible representations;

2. the differential of the action of $L$ is equal to the restriction to $\mathfrak{l}_{0}$ of the action of $\mathfrak{h}_{0}$; and

3. $\left.l \cdot(X \cdot v)=(\operatorname{Ad}(l) X) \cdot(l \cdot v), l \in L, X \in \mathfrak{h}_{0}, v \in V_{L}^{\infty}\right)$; or equivalently

4. $\left.l \cdot(u \cdot v)=(\operatorname{Ad}(l) u) \cdot(l \cdot v), l \in L, u \in U(\mathfrak{h}), v \in V_{L}^{\infty}\right)$.

The Lemma is easy to prove.

Definition 2.10. Suppose $L$ is a compact subgroup of a real Lie group $\{$ def $: g K\}$ $H$. An $\left(\mathfrak{h}_{0}, L\right)$-module is a complex vector space $V$ that is at the same time a representation of the group $L$ and of the real Lie algebra $\mathfrak{h}_{0}$, subject to the conditions in Lemma 2.9. A morphism of $\left(\mathfrak{h}_{0}, L\right)$-modules is a linear map respecting the actions of $L$ and of $\mathfrak{h}_{0}$ separately.

Lemma 2.11. Suppose $L$ is a compact subgroup of a real Lie group $H$. Passage to L-finite smooth vectors

$$
V \rightsquigarrow V_{L}^{\infty}
$$

defines a faithful functor from the category of representations to the category of $\left(\mathfrak{h}_{0}, L\right)$-modules. In particular, any (nonzero) intertwining operator between representations induces a (nonzero) morphism between the corresponding $\left(\mathfrak{h}_{0}, L\right)$-modules.

Again this is very easy. What is much harder (and requires more hypotheses) is proving results in the other direction: that $\left(\mathfrak{h}_{0}, L\right)$-morphisms induce maps between group representations. For that, we return to the setting of a real reductive Lie group $G$. 
Fix once and for all a choice of

$$
K=\text { maximal compact subgroup of } G \text {. }
$$

By a theorem of E. Cartan, this choice is unique up to conjugation. One maximal compact subgroup of $G L(n, \mathbb{R})$ is the orthogonal group $O(n)$. If $G$ is any real reductive subgroup of $G L(n, \mathbb{R})$ that is preserved by transpose, then we may choose $K=G \cap O(n)$ as a maximal compact subgroup of $G$. (This fact applies to the standard matrix realizations of all the classical groups. One can find a proof for example in [19], pages 3-4.) Here are some of Harish-Chandra's fundamental results about quasisimple representations.

Theorem 2.13. Suppose $G$ is a real reductive Lie group and $K$ is a maximal compact subgroup.

\{theorem:quasisimple\}

$\{$ item:segal $\}$

1. Suppose $V$ is an irreducible unitary representation of $G$. Then $V$ is quasisimple.

$\{$ item: $\mathrm{HC} 1\}$

2. Suppose $V$ is an irreducible quasisimple representation of $G$ (Definition 2.3). Then the associated $\left(\mathfrak{g}_{0}, K\right)$-module $V_{K}^{\infty}$ (Lemma 2.9) is irreducible.

3. Suppose $V_{1}$ and $V_{2}$ are irreducible unitary representations of $G$, and that $V_{1, K}^{\infty} \simeq V_{2, K}^{\infty}$ as $\left(\mathfrak{g}_{0}, K\right)$-modules. Then $V_{1} \simeq V_{2}$ as unitary representations.

4. Suppose $X$ is an irreducible $\left(\mathfrak{g}_{0}, K\right)$-module. Then there is an irreducible quasisimple representation $V$ of $G$ so that $X \simeq V_{K}^{\infty}$ as $\left(\mathfrak{g}_{0}, K\right)$ modules.

5. Suppose $X$ is an irreducible $\left(\mathfrak{g}_{0}, K\right)$-module. Then $X$ is associated to an irreducible unitary representation of $G$ if and only if $X$ admits a positive definite invariant Hermitian form $\langle,\rangle_{X}$. Here "invariant" means

$$
\begin{aligned}
\langle k \cdot v, w\rangle_{X}=\left\langle v, k^{-1} \cdot w\right\rangle_{X}, \quad & \langle X \cdot v, w\rangle_{X}=-\langle v, X \cdot w\rangle_{X} \\
& \left(k \in K, X \in \mathfrak{g}_{0}, v, w \in X\right)
\end{aligned}
$$

Proof. Part 1 is due to Segal [34]. Part 2 is proved in Harish-Chandra [9], although it is difficult to point to a precise reference; the result is a consequence of Lemmas 33 and 34 and Theorem 5 (on pages 227-228). Part 3 is Theorem 8 of [9]. Part 4 is Theorem 4 of Harish-Chandra [10]. Part 5 is Theorem 9 of [9] 
Definition 2.14. Suppose $G$ is a real reductive Lie group and $K$ is

a maximal compact subgroup. Two representations $V_{1}$ and $V_{2}$ of $G$ are said to be infinitesimally equivalent if the corresponding $\left(\mathfrak{g}_{0}, K\right)$ modules $V_{1, K}^{\infty}$ and $V_{2, K}^{\infty}$ (Lemma 2.9) are equivalent. We define

$$
\begin{aligned}
\widehat{G} & ={ }_{\text {def }}\{\text { infinitesimal equiv classes of irr quasisimple reps of } G\} \\
& =\left\{\text { equivalence classes of irreducible }\left(\mathfrak{g}_{0}, K\right) \text {-modules }\right\}
\end{aligned}
$$

and call this set the "nonunitary dual of $G$." The $\left(\mathfrak{g}_{0}, K\right)$-module $V_{1, K}^{\infty}$ is called the Harish-Chandra module of $V_{1}$.

Harish-Chandra's theorem 2.13 provides a natural containment of the unitary dual (1.1d) in the nonunitary dual

$$
\widehat{G}_{u} \subset \widehat{G}
$$

always assuming that $G$ is real reductive. In case $G=K$ is compact, then every irreducible representation is finite-dimensional, and the representation space can be given a $K$-invariant Hilbert space structure; so every representation is equivalent to a unitary one, and

$$
\widehat{K}_{u}=\widehat{K}
$$

We are going to recall in Section 6 the results of Langlands and of Knapp-Zuckerman that provide a complete and explicit parametrization of the nonunitary dual $\widehat{G}$. The rest of the paper will be devoted to an algorithm for identifying $\widehat{G}_{u}$ as a subset of these parameters for $\widehat{G}$.

It is well known that in order to discuss real-linear complex represen$\{$ se : cptred $\}$ tations of a real Lie algebra $\mathfrak{h}_{0}$, it is equivalent (and often preferable) to discuss complex-linear representations of the complexified Lie algebra

$$
\mathfrak{h}={ }_{\text {def }} \mathfrak{h}_{0} \otimes_{\mathbb{R}} \mathbb{C} .
$$

We will conclude this section with an equally useful way of speaking about representations of

$$
L=\text { compact Lie group. }
$$

For that, we use the algebra

$$
R(L)=\begin{aligned}
& \text { def } \\
& \text { dimensional representations of } L
\end{aligned}
$$


This is an algebra of functions on $L$ (closed under multiplication) because of the operation of tensor product of representations. It is an elementary exercise in Lie theory that $L$ has a finite-dimensional faithful self-dual representation $\rho$. Write $E_{\rho}$ for the finite-dimensional space of matrix coefficients of $\rho$. The Stone-Weierstrass Theorem guarantees that the algebra generated by $E_{\rho}$ is dense in the continuous functions on $L$. The Schur orthogonality relations then imply that $E_{\rho}$ generates $R(L)$. As an algebra of smooth functions on a compact manifold, $R(L)$ has no nilpotent elements; so $R(L)$ is the algebra of regular functions on a complex affine algebraic variety $L(\mathbb{C})$. The closed points of $L(\mathbb{C})$ are the maximal ideals of $R(L)$, and these include the ideals of functions vanishing at one point of $L$; so

$$
L \subset L(\mathbb{C})
$$

We call $L(\mathbb{C})$ the complexification of $L$.

For the formulation of the next theorem, we need to recall (see for example [5], §§11-14, or [37], Chapter 11) the notion of a real form of a complex affine algebraic variety $X(\mathbb{C})$. Write $R(X)$ for the (complex) algebra of regular functions on $X(\mathbb{C})$. Because $\mathbb{C} / \mathbb{R}$ is a Galois extension, we can define a real form as a certain kind of action of the Galois group on $R(X)$. Since the Galois group has only one nontrivial element, a real form of $X(\mathbb{C})$ is given by a single map (the action of complex conjugation)

$$
\sigma^{*}: R(X) \rightarrow R(X)
$$

The map $\sigma^{*}$ has the characteristic properties

1. $\sigma^{*}$ is a ring automorphism of $R(X)$ :

$$
\sigma^{*}(f g)=\sigma^{*}(f) \sigma^{*}(g), \quad \sigma^{*}(f+g)=\sigma^{*}(f)+\sigma^{*}(g) \quad(f, g \in R(X)) .
$$

2. $\sigma^{*}$ has order 2 .

3. $\sigma^{*}$ is conjugate linear:

$$
\sigma^{*}(z f)=\bar{z} \sigma^{*}(f) \quad(z \in \mathbb{C}, f \in R(X)) .
$$

Then $\sigma^{*}$ defines an automorphism $\sigma$ of order 2 of the set $X(\mathbb{C})$ of maximal ideals in $R(X)$, and the (closed) real points $X(\mathbb{R}, \sigma)$ are the maximal ideals fixed by $\sigma$. Because the ring of functions on a variety has no nilpotent elements, we can recover $\sigma^{*}$ from $\sigma$ : regarding elements of $R(X)$ as functions on $X(\mathbb{C})$, we have

$$
\left(\sigma^{*} f\right)(x)=\overline{f(\sigma(x))} .
$$


Theorem 2.17. Suppose $L$ is a compact Lie group.

1. The construction of (2.15) enlarges $L$ to a complex reductive algebraic group $L(\mathbb{C})$. It is a covariant functor in $L$.

2. Every locally finite continuous representation $\pi$ of $L$ extends uniquely to an algebraic representation $\pi(\mathbb{C})$ of $L(\mathbb{C})$ on the same space; and every algebraic representation of $L(\mathbb{C})$ restricts to a locally finite continuous representation of $L$.

3. There is unique real form $\sigma_{c}$ of $L(\mathbb{C})$ with $L\left(\mathbb{R}, \sigma_{c}\right)=L$.

Conversely, suppose $L(\mathbb{C})$ is a complex reductive algebraic group. There is a real form $\sigma_{c}$ of $L(\mathbb{C})$, unique up to conjugation by $L(\mathbb{C})$, whose group of real points $L=L\left(\mathbb{R}, \sigma_{c}\right)$ is compact, and meets every component of $L(\mathbb{C})$. Then $L(\mathbb{C})$ is the complexification of $L$.

In the setting of the theorem, suppose $(\pi, V)$ is a finite-dimensional unitary representation of $L$ with inner product $\langle$,$\rangle . If u, v \in V$, then the matrix coefficient

$$
f_{u, v}(\ell)={ }_{\operatorname{def}}\langle\pi(\ell) u, v\rangle
$$

is one of the spanning functions used to define $R(L)$. The real form in the theorem is

$$
\left(\sigma^{*} f_{u, v}\right)(\ell)=\left\langle\pi\left(\ell^{-1}\right) v, u\right\rangle .
$$

From this one can see that

$$
L=\left\{\ell \in L(\mathbb{C}) \mid \pi(\mathbb{C})(\ell) \text { is unitary, all } \pi \in \widehat{L}_{u}\right\} .
$$

It follows in particular that $L$ is a maximal compact subgroup of $L(\mathbb{C})$.

Corollary 2.18. Suppose $L$ is a compact subgroup of a real Lie group $H$, and $L(\mathbb{C})$ is the complexification of $L(c f$. (2.15)). Define an $(\mathfrak{h}, L(\mathbb{C}))$ module by analogy with Definition 2.10, requiring the representation of $\mathfrak{h}$ to be complex linear and the action of $L(\mathbb{C})$ to be algebraic. Then any $\left(\mathfrak{h}_{0}, L\right)$ module extends uniquely to an $(\mathfrak{h}, L(\mathbb{C}))$-module; and this extension defines an equivalence of categories from $\left(\mathfrak{h}_{0}, L\right)$-modules to $(\mathfrak{h}, L(\mathbb{C}))$-modules. 


\section{$3 \quad$ Real reductive groups}

Before embarking on more representation theory, we must be more precise about the definition of "reductive group," since the detailed description of the nonunitary dual is sensitive to details of this definition.

We begin with a complex connected reductive algebraic group

$$
G(\mathbb{C})
$$

Recall that this means a subgroup of the group of $n \times n$ invertible matrices, specified as the zero locus of a collection of polynomial equations in the matrix entries and the inverse of the determinant, with two additional properties:

1. $G(\mathbb{C})$ has no nontrivial normal subgroup consisting of unipotent matrices, and

2. $G(\mathbb{C})$ is connected as a Lie group.

The words preceding the conditions are the definition of "complex algebraic;" the first condition is the definition of "reductive;" and the second condition is (equivalent to) the definition of "connected." The first condition may be replaced by

$1^{\prime}$. After an appropriate change of basis in $\mathbb{C}^{n}$, the group $G(\mathbb{C})$ is preserved by the automorphism $\sigma_{c}$,

$$
\sigma_{c}(g)=\left(g^{*}\right)^{-1}
$$

$\{$ sec:realred $\}$

\{se:reductive $\}$ 
(Essentially we are repeating the discussion of real forms from (2.16), but now in the category of analytic manifolds rather than of varieties. For reductive algebraic groups, the two categories give rise to exactly the same real forms.) Here "antiholomorphic" means that if $f$ is a (locally defined) holomorphic function on $G(\mathbb{C})$, then

$$
\left(\sigma^{*} f\right)(g)=\overline{f(\sigma(g))}
$$

is also holomorphic. Clearly the differential at the identity of a real form of $G(\mathbb{C})$ is a real form of the complex Lie algebra $\mathfrak{g}$ : that is, a real Lie algebra automorphism of order two carrying multiplication by $i$ to multiplication by $-i$. (But not every real form of the Lie algebra must exponentiate to the group.)

Given a real form $\sigma$, the group of real points is

$$
G=G(\mathbb{R}, \sigma)=G(\mathbb{C})^{\sigma},
$$

$\{$ eq:Grealpoints $\}$

the group of fixed points of $\sigma$ on $G(\mathbb{C})$. This is a real Lie group of real dimension equal to the complex dimension of $G(\mathbb{C})$. A real reductive algebraic group is a group of real points $G=G(\mathbb{R}, \sigma)$ for a complex connected reductive algebraic group.

This is the class of groups with which we will work in this paper. It is fairly to common to work with a somewhat larger class of groups: to start with $G(\mathbb{R}, \sigma)$ as above, and to allow a real Lie group $\widetilde{G}$ endowed with a homomorphism

$$
\pi: \widetilde{G} \rightarrow G(\mathbb{R}, \sigma)
$$

having finite kernel and open image. For such a group $\widetilde{G}$, it is still possible to formulate the very precise form of the Langlands classification theorem that we need. But the unitarity algorithm will use also the Kazhdan-Lusztig theory of irreducible characters, and this is not available in the setting (3.1f). (A number of interesting special cases have been treated, for example in [29].) Once this Kazhdan-Lusztig theory is available, the rest of the proof of the unitarity algorithm can proceed as in the linear case. But we will not discuss such extensions further.

Example 3.2. Suppose $G(\mathbb{C})$ is a complex connected reductive algebraic group; that is, a subgroup of $G L(n, \mathbb{C})$ satisfying the conditions described above. According to the reformulated definition of reductive, we can (after change of basis in $\mathbb{C}^{n}$ ) assume that $G(\mathbb{C})$ is preserved by the operation $\sigma_{c}(g)=\left(g^{*}\right)^{-1}$, the inverse conjugate transpose of $g$. Clearly $\sigma_{c}$ is an antiholomorphic involution of $G(\mathbb{C})$, so it defines a real form

$$
G\left(\mathbb{R}, \sigma_{c}\right)=G(\mathbb{C}) \cap U(n),
$$


the group of unitary matrices in $G(\mathbb{C})$. Since $G(\mathbb{C})$ is closed in $G L(n, \mathbb{C}$ ) (as the set of zeros of some polynomials), its intersection with $U(n)$ is closed, and therefore compact. This real form is called a compact real form of $G(\mathbb{C})$. It is unique up to conjugation by an inner automorphism of $G(\mathbb{C})$.

Example 3.3. Suppose $G(\mathbb{C})=S O(n, \mathbb{C})$, the group of $n \times n$ orthogonal matrices $\left(g \cdot{ }^{t} g=\mathrm{Id}\right)$ of determinant 1 . Clearly this is an algebraic subgroup of $G L(n, \mathbb{C})$, and clearly it satisfies the second condition $1^{\prime}$ to be reductive. It is not difficult to show that $G(\mathbb{C})$ is connected. Because ${ }^{t} g=g^{-1}$ for $g \in G(\mathbb{C})$, the compact real form acts by $\sigma_{c}(g)=\bar{g}$; so the group of fixed points is

$$
G\left(\mathbb{R}, \sigma_{c}\right)=S O(n)=\{\text { real orthogonal matrices of determinant } 1\} .
$$

To construct other real forms, write $n=p+q$ for nonnegative integers $p$ and $q$, and let $\epsilon(p, q)$ be the diagonal matrix whose first $p$ entries are +1 and whose last $q$ entries are -1 . Then

$$
\sigma(p, q)=\sigma_{c} \circ(\text { conjugation by } \epsilon(p, q))
$$

is an antiholomorphic involutive automorphism of $G(\mathbb{C})$ : a real form. (The key point is that the automorphism $\sigma_{c}$ fixes $\epsilon(p, q)$, so that the two factors in the definition of $\sigma(p, q)$ commute.) A little thought shows that $G(\mathbb{R}, \sigma(p, q))$ consists of the elements of $S O(n, \mathbb{C})$ that are represented by real matrices in the basis $\left\{e_{1}, \ldots, e_{p}, i e_{p+1}, \ldots, i e_{p+q}\right\}$. From this it follows easily that

$$
G(\mathbb{R}, \sigma(p, q)) \simeq S O(p, q),
$$

the group of determinant one real matrices preserving a quadratic form of signature $(p, q)$.

If $n=p+q$ is odd, this turns out to be all the real forms of $S O(n, \mathbb{C})$. If $n$ is even and at least 4 , there are more (perhaps best understood as groups of $(n / 2) \times(n / 2)$ quaternionic matrices $)$.

Part of the point of this example is to understand what groups are being excluded by our convention. One example is the connected semisimple group $S O_{e}(p, q)$, the identity component of $S O(p, q)$. There is no difficulty in formulating the Langlands classification for this group, and the KazhdanLusztig theory works perfectly for it, so our unitarity algorithm could be applied; but for convenience we are excluding this group. (Its unitary representations are precisely the constituents of the restrictions of unitary representations of $S O(p, q)$, so they are not difficult to compute from what we do.) 
A more serious exclusion is (for $p$ and $q$ each at least 3) the four-fold universal cover $\left[S O_{e}(p, q)\right]^{\sim}$. This group is not a matrix group, so extending our algorithm to apply to it requires a nontrivial extension of the KazhdanLusztig theory (parallel to what is done in [29]).

We conclude with a little structure theory for real reductive groups, which will be vital for the formulation of the Langlands classification.

Theorem 3.4 (Cartan; see for example [20], Proposition 1.143, or [28], Theorem 5.1.4). Suppose $G(\mathbb{C})$ is a complex connected reductive algebraic group ((3.1)).

1. Suppose $\sigma_{0}$ is a real form of $G(\mathbb{C})$. Then there is a compact real form $\sigma_{c}$ of $G(\mathbb{C})$ that commutes with $\sigma_{0}$. This compact real form is unique up to conjugation by $G=G\left(\mathbb{R}, \sigma_{0}\right)$. The composition

$$
\theta=\sigma_{0} \circ \sigma_{c}
$$

\{eq: cartaninvolution $\}$

is an algebraic automorphism of $G(\mathbb{C})$ of order two; it is called a Cartan involution for the real form $\sigma_{0}$.

2. Suppose $\theta$ is an involutive algebraic automorphism of $G(\mathbb{C})$. Then there is a compact real form $\sigma_{c}$ of $G(\mathbb{C})$ that commutes with $\sigma_{0}$. This compact real form is unique up to conjugation by $K(\mathbb{C})=G(\mathbb{C})^{\theta}$. The composition

$$
\sigma_{0}=\theta \circ \sigma_{c}
$$

is a real form of $G(\mathbb{C})$, called a real form for the involution $\theta$.

3. The constructions above define a bijection

$$
\begin{aligned}
& \{\text { real forms of } G(\mathbb{C})\} /(\text { conjugation by } G(\mathbb{C})) \\
\leftrightarrow & \{\text { algebraic involutions of } G(\mathbb{C})\} /(\text { conjugation by } G(\mathbb{C}))
\end{aligned}
$$

4. The group $K=G^{\theta}$ is maximally compact in $G$. Its complexification is the reductive algebraic group

$$
K(\mathbb{C})=G(\mathbb{C})^{\theta} .
$$

5. Write

$$
\mathfrak{g}_{0}=\mathfrak{k}_{0}+\mathfrak{s}_{0}
$$

for the decomposition of the real Lie algebra $\mathfrak{g}_{0}=\operatorname{Lie}\left(G\left(\mathbb{R}, \sigma_{0}\right)\right)$ into the +1 and -1 eigenspaces of the Cartan involution $\theta$. (In the setting (3.1b), these are the skew-hermitian and hermitian matrices in 
the Lie algebra, respectively.) Then there is a (K-equivariant under conjugation) diffeomorphism

$$
K \times \mathfrak{s}_{0} \rightarrow G, \quad(k, X) \mapsto k \cdot \exp (X) .
$$

The last assertion is a generalization (and also a consequence) of the polar decomposition for invertible matrices. The fact that $K$ is a maximal compact subgroup follows immediately from this decomposition.

\section{Maximal tori}

Our next serious goal is Langlands' classification of the irreducible (possibly nonunitary) representations of a real reductive group. To a first approximation, it says that the irreducible representations of such a group are indexed by orbits of the (real) Weyl groups on characters of (real points of) maximal tori. What requires a little care is the understanding of "maximal tori;" that is the subject of the present section.

Definition 4.1. Suppose $G(\mathbb{C})$ is a complex connected reductive algebraic group $((3.1))$. A (complex) maximal torus in $G(\mathbb{C})$ is a maximal connected abelian subgroup consisting of diagonalizable matrices. Automatically such a subgroup $H(\mathbb{C})$ is again complex connected reductive algebraic. Its character lattice is the group of algebraic group homomorphisms

$$
X^{*}={ }_{\text {def }} \operatorname{Hom}_{\text {alg }}\left(H(\mathbb{C}), \mathbb{C}^{\times}\right) .
$$

This is a lattice (a finitely-generated torsion free abelian group) of rank equal to the complex dimension of $H(\mathbb{C})$. The dual lattice

$$
X_{*}=_{\text {def }} \operatorname{Hom}\left(X^{*}, \mathbb{Z}\right)
$$

is naturally isomorphic to

$$
X_{*} \simeq \operatorname{Hom}_{\operatorname{alg}}\left(\mathbb{C}^{\times}, H(\mathbb{C})\right),
$$

the lattice of one-parameter subgroups.

Suppose now that $\sigma$ is a real form of $G(\mathbb{C})$, so that $G(\mathbb{R}, \sigma)$ is a real reductive algebraic group. We say that $H(\mathbb{C})$ is defined over $\mathbb{R}$ (with respect to $\sigma$ ) if it is preserved by $\sigma$. In that case the group of real points of $H(\mathbb{C})$ is

$$
H=H(\mathbb{R}, \sigma)=G(\mathbb{R}, \sigma) \cap H(\mathbb{C})=G \cap H(\mathbb{C}),
$$

which we call a (real) maximal torus of $G$. 
All complex maximal tori in $G(\mathbb{C})$ are conjugate, and all are isomorphic to $\left(\mathbb{C}^{\times}\right)^{n}$. There can be more than one conjugacy class of real maximal tori in $G$, and in that case they are not all isomorphic. For example, in the real form $S L(2, \mathbb{R})$ of $S L(2, \mathbb{C})$ there are two real forms of the maximal torus $\mathbb{C}^{\times}$. One is $\sigma(z)=\bar{z}$, with real points $\mathbb{R}^{\times}$; and the other is $\sigma_{c}(z)=(\bar{z})^{-1}$, with real points the (compact) circle group.

Because lattices are built from copies of $\mathbb{Z}$, it is traditional and natural to write the group law in $X^{*}$ as + . But the group law in $X^{*}$ arises from multiplication of the (nonzero complex) values of algebraic characters, so there is always a danger of confusion in the notation. We will try to offer warnings about some instances of this confusion.

The lattice $X^{*}$ is a fundamental invariant of the complex reductive group. Our first goal is to describe all of the (continuous) characters - the possibly nonunitary representations - of a real torus $H$ in terms of this lattice of (algebraic) characters of $H(\mathbb{C})$. (This is a way of thinking about the Langlands classification theorem for the abelian reductive group $H$.) It will be instructive at the same time to identify the unitary characters within these nonunitary representations, since that is the simplest case of the classification of unitary representations at which we are aiming.

Proposition 4.2. Suppose $H(\mathbb{C})$ is a connected abelian complex reductive algebraic group (a complex torus), and $X^{*}$ is the lattice of algebraic characters (Definition 4.1).

1. We can recover $H(\mathbb{C})$ from $X^{*}$ by the contravariant functor

$$
H(\mathbb{C}) \simeq \operatorname{Hom}\left(X^{*}, \mathbb{C}^{\times}\right) .
$$

The complex Lie algebra $\mathfrak{h}$ is

$$
\mathfrak{h} \simeq X_{*} \otimes_{\mathbb{Z}} \mathbb{C}
$$

and therefore is in a natural way defined over $\mathbb{Z}$.

2. Any real form $\sigma$ of $H(\mathbb{C})$ gives rise to an automorphism $\theta$ of order 2 of $X^{*}$, by the requirement

$$
\theta(\lambda)(h)=\overline{\lambda(\sigma h)}^{-1}
$$

This defines a bijection between real forms and automorphisms of order two of $X^{*}$. 
3. An automorphism $\theta$ of $X^{*}$ of order two induces an automorphism (still called $\theta$ ) of $H(\mathbb{C})$ of order two, by means of the functor in (1). This automorphism is the Cartan involution corresponding to $\sigma$ (Theorem 3.4). In particular, $\theta$ is trivial if and only if $H(\mathbb{R}, \sigma)$ is compact.

Proposition 4.3. Suppose $H(\mathbb{C})$ is a connected abelian complex reductive \{prop:toruschars\} algebraic group (a complex torus), $X^{*}$ is the lattice of algebraic characters (Definition 4.1), and $\sigma$ is a real form of $H(\mathbb{C})$. Write $\theta$ for the corresponding Cartan involution (Proposition 4.2), and $H=H(\mathbb{R}, \sigma)$ for the group of real points.

1. The group

$$
T=H^{\theta}
$$

is the (unique) maximal compact subgroup of $H$. Its complexification is the (possibly disconnected) reductive algebraic group

$$
T(\mathbb{C})=H(\mathbb{C})^{\theta} .
$$

2. Write

$$
\mathfrak{h}_{0}=\mathfrak{t}_{0}+\mathfrak{a}_{0}
$$

for the decomposition of the real Lie algebra of $H$ into +1 and -1 eigenspaces of $\theta$. Then

$$
\begin{aligned}
\mathfrak{t}_{0} & \simeq \operatorname{Hom}_{\mathbb{Z}}\left(\left(X^{*}\right)^{\theta}, i \mathbb{R}\right), \\
\mathfrak{a}_{0} & \simeq \operatorname{Hom}_{\mathbb{Z}}\left(\left(X^{*}\right)^{-\theta}, \mathbb{R}\right) .
\end{aligned}
$$

3. Write

$$
A=\exp \left(\mathfrak{a}_{0}\right),
$$

the identity component of the maximal split torus of $H$ : the identity component of the subgroup of elements $h$ with $\theta h=h^{-1}$. The group $A$ is isomorphic to its Lie algebra, and

$$
H \simeq T \times A,
$$

a direct product of abelian Lie groups.

4. The group of continuous characters of $T$ may be identified with the group of algebraic characters of $T(\mathbb{C})$, and so (by restriction) with a quotient of $X^{*}$ :

$$
\widehat{T} \simeq \operatorname{Hom}_{a l g}\left(T(\mathbb{C}), \mathbb{C}^{\times}\right) \simeq X^{*} /(1-\theta) X^{*} .
$$


5. The group of continuous characters of $A$ is

$$
\widehat{A} \simeq \mathfrak{a}^{*} \simeq\left(X^{*}\right)^{-\theta} \otimes_{\mathbb{Z}} \mathbb{C},
$$

a complex vector space naturally defined over $\mathbb{Z}$. Therefore

$$
\widehat{H} \simeq \widehat{T} \times \widehat{A} \simeq\left[X^{*} /(1-\theta) X^{*}\right] \times\left[\left(X^{*}\right)^{-\theta} \otimes_{\mathbb{Z}} \mathbb{C}\right],
$$

a direct product of a finitely generated discrete abelian group and a complex vector space defined over $\mathbb{Z}$.

6. Suppose $\gamma=(\lambda, \nu) \in \widehat{T} \times \widehat{A}$ is a parameter for a character of $H$. Then the character is unitary if and only if $\nu$ is purely imaginary; that is,

$$
\nu \in\left(X^{*}\right)^{-\theta} \otimes_{\mathbb{Z}} i \mathbb{R} .
$$

There is an essential asymmetry between the two factors $T$ and $A$ in part (3). The first is the group of real points of an algebraic group. The symmetrically defined algebraic group

$$
A(\mathbb{C})=H(\mathbb{C})^{-\theta}
$$

is also defined over $\mathbb{R}$, and its group of real points is

$$
A(\mathbb{R})=\left\{h \in H \mid \theta(h)=h^{-1}\right\} .
$$

This group is often disconnected; and

$$
A=\text { identity component of } A(\mathbb{R}) \text {. }
$$

(This notation is inconsistent with our general practice of writing unadorned Roman letters for groups of real points; we will not make use of the algebraic group $A(\mathbb{C})$, so no confusion should arise.)

Proof. Parts (1)-(3) are just the Cartan decomposition of Theorem 3.4 for the abelian case. The first assertion in part (4) (identifying continuous representations of a compact Lie group with algebraic representations of its complexification) is very general, and in some sense amounts to the way the complexification is defined. The second part of (4) is part of the general fact that taking algebraic characters is a contravariant equivalence of categories from complex reductive abelian groups to finitely generated abelian groups.

For (5), the characters of a vector group are the same thing as complexvalued linear functionals on the Lie algebra; this can be computed from the 
description of the Lie algebra in (2). The linear functional $\nu$ corresponds to the character $e^{\nu}$ defined by

$$
e^{\nu}(\exp X)=e^{\nu(X)} \quad\left(X \in \mathfrak{a}_{0}\right) .
$$

This formula makes it plain that the character $e^{\nu}$ is unitary if and only if $\nu$ takes purely imaginary values. (Any character of the compact abelian group $T$ is automatically unitary.)

We see therefore that a parameter for a character of $H$ has a discrete part (the restriction to $T$, given by an element of the lattice quotient $X^{*} /(1-$ $\theta) X^{*}$ ) and a continuous part (the restriction to $A$, given by an element of the complex vector space $\left.\mathfrak{a}^{*}\right)$. The complex vector space is naturally defined over $\mathbb{R}$ (with real points $\mathfrak{a}_{0}^{*}$ ); and the character is unitary if and only if the real part of the continuous parameter is equal to zero. These statements are the ones we will generalize to all real reductive groups.

\section{Coverings of tori}

What enters most naturally in the Langlands classification are characters not of the real tori $H$, but of certain double covers

$$
1 \rightarrow\{1, \epsilon\} \rightarrow \widetilde{H} \rightarrow H \rightarrow 1 .
$$

The reason is that we are interested in versions of the Weyl character formula, and the Weyl denominator is a function not on the torus but rather on such a double cover. Here is the construction we need.

Definition 5.1. Suppose $H$ is a Lie group, $n$ is a positive integer, and

$$
\gamma \in \operatorname{Hom}\left(H, \mathbb{C}^{\times}\right)
$$

an element of the group of (one-dimensional) continuous characters of $H$. The $\gamma / n$ cover of $H$ is the Lie group

$$
\widetilde{H}=\left\{(h, z) \in H \times \mathbb{C}^{\times} \mid \gamma(h)=z^{n}\right\} .
$$

(Here $\gamma / n$ is for the moment a formal symbol, recording the defining character $\gamma$ and the level $n$ of the cover. Despite the absence of $\gamma$ and $n$ in the notation, the group $\widetilde{H}$ depends very much on both of these values.) Projection on the first factor defines a surjective homomorphism 
to $H$; the kernel is isomorphic (by projection on the second factor) to the group

$$
\left\langle\zeta_{n}\right\rangle=\left\{1, \zeta_{n}, \zeta_{n}^{2}, \ldots, \zeta_{n}^{n-1}\right\}
$$

(with $\zeta_{n}=e^{2 \pi i / n}$ ) of $n$th roots of 1 in $\mathbb{C}^{\times}$. Therefore $\widetilde{H}$ is an $n$-fold cover of $H$, in the sense that there is a natural short exact sequence

$$
1 \rightarrow\left\langle\zeta_{n}\right\rangle \rightarrow \widetilde{H} \rightarrow H \rightarrow 1
$$

The subgroup $\left\langle\zeta_{n}\right\rangle$ is central. Projection on the second factor defines a group character

$$
(\gamma / n): \widetilde{H} \rightarrow \mathbb{C}^{\times}, \quad(\gamma / n)(h, z)=z .
$$

The $n$th power of this character descends to $H$, and is equal to $\gamma$; that is why we call $\widetilde{H}$ the " $\gamma / n$ cover."

Any (reasonable; for example, if $H$ is reductive, "quasisimple" is a strong enough hypothesis) irreducible representation $\pi$ of $\widetilde{H}$ must send $\zeta_{n}$ to $\zeta_{n}^{k}$ for some $k \in \mathbb{Z}$; we say that such a representation has level $k$. It is easy to check that all level $k$ representations are uniquely of the form $\tau \otimes k(\gamma / n)$ for some $\tau \in \widehat{H}$ :

$$
(\widetilde{H})_{k}=\text { def }\{\text { level } k \text { irreducibles of } \widetilde{H}\} \leftrightarrow k(\gamma / n) \otimes \widehat{H} .
$$

An expression $\gamma_{2}=\gamma_{1}+n \phi$ for one-dimensional characters of $H$ gives rise to a natural isomorphism

$$
\widetilde{H}\left(\gamma_{1} / n\right) \simeq_{\phi} \widetilde{H}\left(\gamma_{2} / n\right), \quad(h, z) \mapsto(h, z \phi(h)) .
$$

In order to introduce the coverings we need, we recall now the fundamental structure theory of complex reductive groups.

Definition 5.2. Suppose $G(\mathbb{C})$ is a complex connected reductive alge$\{$ def : posroots $\}$ braic group, $H(\mathbb{C})$ is a maximal torus, and $X^{*}$ is the lattice of algebraic characters of $H(\mathbb{C})$. Write

$$
R=R(G(\mathbb{C}), H(\mathbb{C})) \subset X^{*}
$$

for the finite set of nonzero weights of the adjoint representation of $H(\mathbb{C})$ on $\mathfrak{g}$, the roots of $H(\mathbb{C})$ in $G(\mathbb{C})$. Attached to each root $\alpha$ there is a three-dimensional subgroup

$$
\phi_{\alpha}: S L(2, \mathbb{C}) \rightarrow G(\mathbb{C}),
$$


an algebraic group homomorphism with finite kernel satisfying

$$
\phi_{\alpha}(\text { diagonal matrices }) \subset H(\mathbb{C}), \quad 0 \neq d \phi_{\alpha}\left(\begin{array}{ll}
0 & 1 \\
0 & 0
\end{array}\right) \in \mathfrak{g}_{\alpha} .
$$

These conditions characterize $\phi_{\alpha}$ uniquely up to conjugation by diagonal matrices in $S L(2, \mathbb{C})$, or by $H(\mathbb{C})$. In particular, they characterize uniquely the coroot $\alpha^{\vee}$ corresponding to $\alpha$,

$$
\alpha^{\vee}: \mathbb{C}^{\times} \rightarrow H(\mathbb{C}), \quad \alpha^{\vee}(z)={ }_{\operatorname{def}} \phi_{\alpha}\left(\left(\begin{array}{cc}
z & 0 \\
0 & z^{-1}
\end{array}\right)\right) .
$$

Write

$$
R^{\vee}=R^{\vee}(G(\mathbb{C}), H(\mathbb{C})) \subset X_{*}
$$

(notation as in Definition 4.1) for the set of coroots.

Attached to the root $\alpha$ is the root reflection

$$
s_{\alpha} \in \operatorname{Aut}\left(X^{*}\right), \quad s_{\alpha}(\lambda)=\lambda-\left\langle\lambda, \alpha^{\vee}\right\rangle \alpha .
$$

This reflection acts also (by transpose) on $X_{*}$, and by extension of scalars on $\mathfrak{h}^{*}=X^{*} \otimes_{\mathbb{Z}} \mathbb{C}$ and $\mathfrak{h}$ and $H(\mathbb{C})$. The group generated by all reflections is the complex Weyl group

$$
W(G(\mathbb{C}), H(\mathbb{C}))=\left\langle s_{\alpha} \mid \alpha \in R(G, H)\right\rangle \subset \operatorname{Aut}\left(X^{*}\right) .
$$

This group may also be described as

$$
W(G(\mathbb{C}), H(\mathbb{C}))=N_{G(\mathbb{C})}(H(\mathbb{C})) / H(\mathbb{C}) \subset \operatorname{Aut}(H(\mathbb{C})),
$$

the group of automorphisms of $H(\mathbb{C})$ arising from $G(\mathbb{C})$.

Suppose $R^{+} \subset R$ is a choice of positive roots (see [15], 10.1). Define

$$
2 \rho=2 \rho\left(R^{+}\right)=\sum_{\alpha \in R^{+}} \alpha \in X^{*}
$$

Write $\Pi=\Pi\left(R^{+}\right)$for the corresponding set of simple roots ([15], 10.1). Then ([15], Lemma 13.3A)

$$
\Pi=\left\{\alpha \in R \mid\left\langle 2 \rho, \alpha^{\vee}\right\rangle=2\right\} .
$$

Consequently all coroots take nonzero even integer values on $2 \rho$; the positive coroots are those taking positive values on $2 \rho$; and the simple coroots are those taking the value 2 on $2 \rho$. 
We pause here for a moment to recall Harish-Chandra's description of the center of the enveloping algebra $\mathfrak{Z}(\mathfrak{g})(2.1)$.

Definition 5.3. In the setting of Definition 5.2, suppose $R^{+}$is a choice of positive roots. Write

$$
\begin{aligned}
\mathfrak{n} & =\mathfrak{n}\left(R^{+}\right)=\sum_{\alpha \in R^{+}} \mathfrak{g}_{\alpha} \\
\mathfrak{n}^{-} & =\mathfrak{n}\left(-R^{+}\right)=\sum_{\alpha \in R^{+}} \mathfrak{g}_{-\alpha}, \\
\mathfrak{b} & =\mathfrak{b}\left(R^{+}\right)=\mathfrak{h}+\mathfrak{n}
\end{aligned}
$$

for the corresponding maximal nilpotent and Borel subalgebras, so that

$$
\mathfrak{g}=\mathfrak{n}^{-} \oplus \mathfrak{h} \oplus \mathfrak{n}
$$

is a triangular decomposition. The Poincaré-Birkhoff-Witt theorem then decomposes the universal enveloping algebra as a vector space

$$
U(\mathfrak{g})=U\left(\mathfrak{n}^{-}\right) \otimes U(\mathfrak{h}) \otimes U(\mathfrak{n}), \quad U(\mathfrak{g})=U\left(\mathfrak{b}^{-}\right) \oplus U(\mathfrak{g}) \mathfrak{n} .
$$

Write

$$
\tilde{\xi}: U(\mathfrak{g}) \rightarrow U\left(\mathfrak{b}^{-}\right)
$$

for the (linear) projection corresponding to the last direct sum decomposition. Then Harish-Chandra observes that the direct sum is preserved by the adjoint action of $\mathfrak{h}$, and therefore

$$
\tilde{\xi}: U(\mathfrak{g})^{\mathfrak{h}} \rightarrow U\left(\mathfrak{b}^{-}\right)^{\mathfrak{h}}=U(\mathfrak{h}) .
$$

Furthermore this restriction is an algebra homomomorphism, the unnormalized Harish-Chandra homomorphism. Since $\mathfrak{h}$ ) is an abelian Lie algebra,

$$
U(\mathfrak{h}) \simeq S(\mathfrak{h}) \simeq \text { polynomial functions on } \mathfrak{h}^{*}
$$

so every $\sigma \in \mathfrak{h}^{*}$ gives rise to an algebra automorphism of $U(\mathfrak{h})$

$$
\tau_{\sigma}=\text { translation by } \sigma, \quad \tau_{\sigma}(Z)=Z-\sigma(Z) \quad(Z \in \mathfrak{h}) .
$$

The normalized Harish-Chandra homomorphism is

$$
\xi: U(\mathfrak{g})^{\mathfrak{h}} \rightarrow U(\mathfrak{h}), \quad \xi(z)=\tau_{\rho}(\tilde{\xi}(z)) .
$$


Here $\rho \in \mathfrak{h}^{*}$ (Definition 5.2) is half the sum of the roots in $R^{+}$. For any $\lambda \in \mathfrak{h}^{*}$, the infinitesimal character $\lambda$ is the algebra homomorphism

$$
\xi_{\lambda}: \mathfrak{Z}(\mathfrak{g}) \rightarrow \mathbb{C}, \quad \xi_{\lambda}(z)=\xi(z)(\lambda)=\tilde{\xi}(z)(\lambda-\rho) .
$$

Here we identify $U(\mathfrak{h})$ with polynomial functions on $\mathfrak{h}^{*}$.

Theorem 5.4 (Harish-Chandra; see [15], 130-134). . Suppose $H(\mathbb{C}) \subset$ $G(\mathbb{C})$ is a maximal torus in a connected reductive algebraic group; use the notation of Definition 5.3. The Harish-Chandra homomorphism $\xi$ is an algebra isomorphism

$$
\xi: \mathfrak{Z}(\mathfrak{g}) \rightarrow U(\mathfrak{h})^{W(G(\mathbb{C}), H(\mathbb{C})}
$$

\section{Consequently}

1. the homomorphism $\xi$ is independent of the choice of positive root system in its definition;

2. every algebra homomorphism from $\mathfrak{Z}(\mathfrak{g})$ to $\mathbb{C}$ is of the form $\xi_{\lambda}$ for some $\lambda \in \mathfrak{h}^{*} ;$ and

3. $\xi_{\lambda}=\xi_{\lambda^{\prime}}$ if and only if $\lambda^{\prime} \in W(G(\mathbb{C}), H(\mathbb{C})) \cdot \lambda$.

Algebraically the most complicated questions in representation theory concern the integral weights $X^{*} \subset \mathfrak{h}^{*}$. The key to the arguments of this paper is deformation: understanding the behavior of representation theory in one-parameter families. Because of these two facts, we will make a great deal of use of

$\{$ def :realinf $\}$

Definition 5.5 ([38], Definition 5.4.11). Suppose we are in the setting of Definition 5.2. Recall from Proposition 4.2 the identifications

$$
\mathfrak{h} \simeq X_{*} \otimes_{\mathbb{Z}} \mathbb{C}, \quad \mathfrak{h}^{*} \simeq X^{*} \otimes_{\mathbb{Z}} \mathbb{C}
$$

exhibiting $\mathfrak{h}$ as canonically defined over $\mathbb{Z}$. This means in particular that $\approx$ is canonically defined over $\mathbb{R}$; we write

$$
\mathfrak{h}_{\mathrm{RE}}=X_{*} \otimes_{\mathbb{R}}, \quad \mathfrak{h}_{\mathrm{RE}}^{*}=X^{*} \otimes_{\mathbb{R}},
$$

the canonical real form of $\mathfrak{h}$. Clearly these spaces are preserved by the action of the Weyl group. The infinitesimal character $\xi_{\lambda}$ is said to be real if $\lambda \in \mathfrak{h}_{\mathrm{RE}}^{*}$. By Theorem 5.4(3), this property is independent of the choice of $\lambda$ representing the infinitesimal character. 
A real infinitesimal character must indeed be real-valued on the form of $\mathfrak{Z}(\mathfrak{g})$ defined by the split real form of $G(\mathbb{C})$, but this is neither a sufficient condition to be real nor (as far as we know) an interesting one.

Lemma 5.6. In the setting of Definition 5.2, suppose $R^{+}$and $\left(R^{+}\right)^{\prime}$ are two \{lemma:wrho\} choices of positive root system, with corresponding sums of positive roots $2 \rho$ and $2 \rho^{\prime}$.

1. There is a homomorphism

$$
\epsilon: W(G(\mathbb{C}), H(\mathbb{C})) \rightarrow\{ \pm 1\}, \quad \epsilon\left(s_{\alpha}\right)=-1 \quad(\alpha \in R) .
$$

2. There is a unique element $w \in W(G, H)$ (depending on $R^{+}$and $\left.\left(R^{+}\right)^{\prime}\right)$ such that $w\left(R^{+}\right)=\left(R^{+}\right)^{\prime}$.

3. Define

$$
S=\left\{\alpha \in R^{+} \mid \alpha \notin\left(R^{+}\right)^{\prime}\right\}, \quad 2 \rho(S)=\sum_{\alpha \in S} \alpha \in X^{*} .
$$

Then $2 \rho^{\prime}=2 \rho-4 \rho(S)$.

4. The $\rho$ and $\rho^{\prime}$ double covers of $H(\mathbb{C})$ (Definition 5.1) are canonically isomorphic. We may therefore speak of "the" $\rho$ double cover of $H(\mathbb{C})$ without reference to a particular choice of positive root system.

The notation introduced in Definition 5.1 says that we should call the coverings in (4) the " $2 \rho / 2$ " and " $2 \rho^{\prime} / 2$ " covers. We prefer either to think of $\rho$ as an abbreviation for the formal symbol $2 \rho / 2$, or else simply to abuse notation in this way. Consistent with this convention, we will also write $\rho$ for the character of this double cover that is called $2 \rho / 2$ in Definition 5.1.

Proof. Part (1) is [15], Exercise 6 on page 54. Part (2) is [15], Theorem 10.3. (Notice that this says that $W(G, H)$ acts in a simply transitive way on positive root systems for $R$.) Part (3) is almost obvious: what matters is that the roots appear in pairs $\{ \pm \alpha\}$, and that a positive system picks exactly one root from each pair. Part (4) follows from (3) and from the discussion at the end of Definition 5.1.

For real groups, the character $\rho$ is not quite the most useful one. In Mackey's definition of unitary induction there appears the square root of the absolute value of the determinant of an action of a reductive subgroup 
(on the cotangent space of a real flag variety). This determinant is essentially a sum of positive roots, so the square root gives something close to $\rho$; but the absolute value has no parallel in what we have done so far. In [1] there is a systematic use of the " $\rho$ double cover" of maximal tori. The absence of Mackey's absolute value requires a complicated correction (see Definition 11.6 of [1]). In this paper we will adopt a slightly different approach, introducing a slightly complicated modification of $\rho$ to define the covering, and as a reward getting a simpler formulation of the Langlands classification in the next section.

$\{$ def :rhoim $\}$

Definition 5.7. Suppose $G(\mathbb{C})$ is complex connected reductive algebraic group endowed with a real form $\sigma_{0}$ (cf. (3.1)) and a Cartan involution $\theta$ (Theorem 3.4). Suppose that $H(\mathbb{C})$ is a maximal torus defined over $\mathbb{R}$. After conjugating by $G$, we may assume that $H$ is also preserved by $\theta$; the shorthand is that " $H$ is a $\theta$-stable maximal torus in G." Define

$$
W(G, H)=N_{G}(H) / H \simeq N_{K}(H) / H \cap K \subset \operatorname{Aut}(H),
$$

the real Weyl group of $H$ in $G$. Because of the second description of the complex Weyl group in Definition 5.2, it is clear that $W(G, H) \subset$ $W(G(\mathbb{C}), H(\mathbb{C}))$.

Write

$$
R=R(G(\mathbb{C}), H(\mathbb{C})) \subset X^{*}
$$

for the roots (Definition 5.2). These roots fall into three classes (each of which is preserved by the real Weyl group $W(G, H))$.

a) the real roots $R_{\mathbb{R}}$, those defining real-valued characters $\alpha$ of $H$ (or equivalently of the Lie algebra $\mathfrak{h}_{0}$ ). Equivalent conditions are

$$
\sigma_{0}(\alpha)=\alpha, \quad \theta(\alpha)=-\alpha .
$$

The real roots are a root subsystem, a Levi factor of $R$.

b) the imaginary roots $R_{i \mathbb{R}}$, those defining unitary characters $\beta$ of $H$ (or equivalently, purely imaginary-valued characters of the Lie algebra $\mathfrak{h}_{0}$ ). Equivalent conditions are

$$
\sigma_{0}(\beta)=-\beta, \quad \theta(\beta)=\beta .
$$

The imaginary roots are a root subsystem, a Levi factor of $R$. 
c) the complex roots $R_{\mathbb{C}}$, those which are neither real-valued nor unitary. Equivalently, these are the roots taking neither real nor purely imaginary values on the Lie algebra $\mathfrak{h}_{0}$. Complex roots appear in four-tuples of distinct roots

$$
\left\{\delta, \sigma_{0}(\delta),-\delta,-\sigma_{0}(\delta)\right\}=\{\delta,-\theta(\delta),-\delta, \theta(\delta)\} .
$$

Recall from Definition 5.2 the algebraic group homomorphism

$$
\phi_{\alpha}: S L(2, \mathbb{C}) \rightarrow G(\mathbb{C})
$$

If $\alpha$ is real or imaginary, then the real form $\sigma_{0}$ and the Cartan involution $\theta$ both preserve the image of $\phi_{\alpha}$. It follows easily that these automorphisms pull back to a real form $\sigma_{\alpha}$ of $S L(2, \mathbb{C})$ and a corresponding Cartan involution $\theta_{\alpha}$ :

$\phi_{\alpha}\left(\sigma_{\alpha}(g)\right)=\sigma_{0}\left(\phi_{\alpha}(g)\right), \quad \phi_{\alpha}\left(\theta_{\alpha}(g)\right)=\theta\left(\phi_{\alpha}(g)\right) \quad(g \in S L(2, \mathbb{C})$.

If $\alpha$ is imaginary, then the diagonal torus in $S L(2, \mathbb{C})$ is compact for $\sigma_{\alpha}$. We may conjugate $\phi_{\alpha}$ so that $\sigma_{\alpha}$ defines either $S U(1,1)$ (in which case we say that $\alpha$ is noncompact) or $S U(2)$ (in which case $\alpha$ is called compact). If $\alpha$ is real, then the diagonal torus is split, and we may conjugate $\phi_{\alpha}$ so that $\sigma_{\alpha}$ defines the real form $S L(2, \mathbb{R})$.

If $\alpha$ is a real or imaginary root, define

$$
m_{\alpha}=\phi_{\alpha}\left(\begin{array}{cc}
-1 & 0 \\
0 & -1
\end{array}\right)=\alpha^{\vee}(-1)
$$

an element of order (one or) two in $T$.

If $\delta$ is complex, the sum $\delta+\sigma_{0}(\delta)$ takes positive real values on $H$, and therefore has a distinguished positive-valued square root

$$
\frac{1}{2}\left(\delta+\sigma_{0}(\delta)\right) \in \widehat{H} .
$$

Similarly, the difference $\delta-\sigma_{0}(\delta)$ is a unitary character of $H$, and has a distinguished unitary square root

$$
\frac{1}{2}\left(\delta-\sigma_{0}(\delta)\right)=_{\operatorname{def}} \delta-\left(\frac{1}{2}\left(\delta+\sigma_{0}(\delta)\right)\right) \in \widehat{H} .
$$

We will eventually need Knapp's detailed description of the real Weyl group $W(G, H)$. The notation can be a little confusing: the complex roots $R_{\mathbb{C}}$ of Definition 5.7 are not a root system, but in this proposition we will consider a (small) subset $R_{C}$ that is a root system. 
Proposition 5.8 (Knapp; see [42], Proposition 4.16). With notation as \{prop:realweyl in Definition 5.7, fix positive root systems $R_{\mathbb{R}}^{+}$and $R_{i \mathbb{R}}^{+}$for the real and imaginary roots, with corresponding half sums $\rho_{\mathbb{R}}$ and $\rho_{i \mathbb{R}}$ in $\mathfrak{h}^{*}$. Define

$$
R_{C}=\left\{\alpha \in R \mid \rho_{\mathbb{R}}\left(\alpha^{\vee}\right)=\rho_{i \mathbb{R}}\left(\alpha^{\vee}\right)=0\right\} .
$$

1. The root system $R_{C}$ is $\theta$-stable and complex ([42], Definition 3.10). That is, it is the sum of two root systems $R_{C}^{L}$ and $R_{C}^{R}=\theta\left(R_{C}^{L}\right)$ that are interchanged by $\theta$.

2. We have

$$
W\left(R_{C}\right)^{\theta}=\left\{(w, \theta w) \mid w \in W\left(R_{C}^{L}\right)\right\} \subset W\left(R_{C}^{L}\right) \times W\left(R_{C}^{R}\right) ;
$$

this description shows that

$$
W\left(R_{C}\right)^{\theta} \simeq W\left(R_{C}^{L}\right)
$$

3.

$$
W(G(\mathbb{C}), H(\mathbb{C}))^{\theta}=W\left(R_{C}\right)^{\theta} \ltimes\left[W\left(R_{\mathbb{R}}\right) \times W\left(R_{i \mathbb{R}}\right)\right],
$$

a semidirect product with the subgroup in brackets normal.

4. The real Weyl group $W(G, H)$ is a subgroup of $W(G(\mathbb{C}), H(\mathbb{C}))^{\theta}$. The first two factors in (3) are contained in $W(G, H)$. Consequently

$$
W(G, H)=W\left(R_{C}\right)^{\theta} \ltimes\left[W\left(R_{\mathbb{R}}\right) \times W\left(G^{A}, H\right)\right] .
$$

Here

$$
W\left(G^{A}, H\right)=W\left(R_{i \mathbb{R}}\right) \cap W(G, H)
$$

is the subgroup of the imaginary Weyl group having representatives in $G$ (or equivalently in $K$ ).

In general we follow a long tradition and write the group structure on one-dimensional characters additively, even though values of the character are being multiplied. In Lie theory this is particularly attractive when we are identifying certain characters (like roots) with linear functionals on the Lie algebra; we write addition on either side of this identification, even though there is an exponential map involved. But in the following lemma we are taking absolute values of some roots, so the tradition would require us to write addition of absolute values when we mean multiplication. This exceeds our (admittedly large) capacity to tolerate abuse of notation. We will therefore write group characters multiplicatively in this lemma. 
Lemma 5.9. In the setting of Definition 5.7, suppose $R^{+}$is a choice of positive root system.

1. Define a character $2 \rho_{\mathrm{abs}}=2 \rho_{\mathrm{abs}}\left(R^{+}\right)$of $H$ by

$$
2 \rho_{\mathrm{abs}}(h)=\left(\prod_{\alpha \in R_{\mathbb{R}}^{+}}|\alpha(h)|\right)\left(\prod_{\beta \in R_{i \mathbb{R}}^{+}} \beta(h)\right)\left(\prod_{\delta \in R_{\mathbb{C}}^{+}} \delta(h)\right)
$$

This character differs only by factors of \pm 1 from $2 \rho$; in particular, it has the same differential as $2 \rho$.

2. In the definition of the character $2 \rho_{\mathrm{abs}}$, each factor in the first product has a distinguished square root $|\alpha|^{1 / 2}$; and the factors in the third product fall into pairs $\left\{\delta, \sigma_{0}(\delta)\right\}$ or $\left\{\delta,-\sigma_{0}(\delta)\right\}$. The product of each such pair of factors has a distinguished square root (Definition 5.7). Consequently the quotient $\left[2 \rho_{\mathrm{abs}}\right]\left[2 \rho_{i \mathbb{R}}\right]^{-1}$ (the second factor being the inverse of the product of the positive imaginary roots) has a distinguished square root.

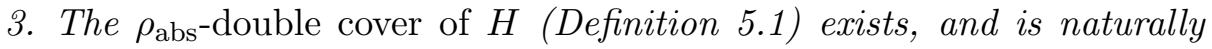
isomorphic to the $\rho_{i \mathbb{R}}$-cover defined by the "half sum" (that is, the square root of the product) of positive imaginary roots.

4. There is a homomorphism

$$
\begin{gathered}
\epsilon_{i \mathbb{R}}: W(G, H) \rightarrow\{ \pm 1\}, \\
\epsilon_{i \mathbb{R}}(w)=(-1)^{\#\{\text { imaginary roots changing sign under } w\}} .
\end{gathered}
$$

5. Suppose $\left(R^{+}\right)^{\prime}$ is a second choice of positive roots. Define

$$
\begin{gathered}
S=\left\{\alpha \in R^{+} \mid \alpha \notin\left(R^{+}\right)^{\prime}\right\}=S_{\mathbb{R}} \cup S_{i \mathbb{R}} \cup S_{\mathbb{C}} \\
2 \rho_{\mathrm{abs}}(S)=\prod_{\alpha \in S_{\mathbb{R}}}|\alpha| \cdot \prod_{\beta \in S_{i \mathbb{R}} \cup S_{\mathbb{C}}} \beta \in \widehat{H} .
\end{gathered}
$$

Then $2 \rho_{\mathrm{abs}}^{\prime}=2 \rho_{\mathrm{abs}} \cdot\left[2 \rho_{\mathrm{abs}}(S)\right]^{-2}$.

6. The $\rho_{\mathrm{abs}}$ and $\rho_{\mathrm{abs}}^{\prime}$ double covers of $H$ (Definition 5.1) are canonically isomorphic. We may therefore speak of "the" $\rho_{\text {abs }}$ double cover of $H$ without reference to a particular choice of positive root system. 
The proof of Lemma 5.9 is parallel to that of Lemma 5.6. We omit the details.

Definition 5.10. Suppose we are in the setting of Definition 5.7. Write $\widetilde{H}$ for the $\rho_{\text {abs }}$-double cover of $H$,

$$
1 \rightarrow\{1,-1\} \rightarrow \widetilde{H} \rightarrow H \rightarrow 1
$$

as in Lemma 5.9. Fix a positive root system $R^{+}$for $H$ in $\mathfrak{g}$, and therefore a character

$$
\rho_{\text {abs }}: \widetilde{H} \rightarrow \mathbb{C}^{\times} .
$$

A Weyl denominator function is the level one (that is, the central element -1 acts by -1 ) function

$$
D(\widetilde{h})=\rho_{\mathrm{abs}}(\widetilde{h}) \cdot\left(\prod_{\alpha \in R_{\mathbb{R}}^{+}}\left|1-\alpha(h)^{-1}\right|\right)\left(\prod_{\beta \in R^{+}-R_{\mathbb{R}}^{+}}\left(1-\beta(h)^{-1}\right)\right) .
$$

(Here $h \in H$ is the image of $\widetilde{h}$ under the covering map.) From this formula it is clear that $D(\widetilde{h}) \neq 0$ if and only if $\widetilde{h}$ is regular; that is, if and only if $\alpha(h) \neq 1$ for all $\alpha \in R$.

An easy calculation shows that replacing $R^{+}$by a different positive system $\left(R^{+}\right)^{\prime}$ changes $D$ by a sign (namely -1 to the number of non-real roots that change sign). Consequently there are at most two possible Weyl denominator functions on each Cartan subgroup, differing by a sign.

The absolute values appearing in the product above are in some sense not so important. We will be using the Weyl denominator to describe character formulas, and these formulas take their nicest form on the subset

$$
H^{+}=\left\{h \in H|| \alpha(h) \mid \geq 1 \quad\left(\alpha \in R_{\mathbb{R}}^{+}\right)\right\} .
$$

(Notice that every $W\left(R_{\mathbb{R}}\right)$-orbit in $H$ must meet $H^{+}$.) On $H^{+}$, all of the factors $1-\alpha(h)^{-1}$ (for $\alpha \in R_{\mathbb{R}}^{+}$) are nonnegative; so the absolute value could be omitted there.

With the Weyl denominator functions in hand, we can begin to understand the nature of the parameters appearing in the Langlands classification of irreducible representations. Perhaps the easiest way to describe the Langlands classification (if not the easiest way to prove it) is by Harish-Chandra's theory of distribution characters. In order to talk about distributions on manifolds, where there is no natural identification of functions with smooth 
measures, it is useful to be a little careful. Recall first of all that a test density on a manifold $M$ is a compactly supported complex-valued smooth measure $T$ on $M$. A generalized function on $M$ is a continuous linear functional on the space of test densities. For example, any locally $L^{1}$ measurable function $f$ on $M$ defines a generalized function, by the rule

$$
f(T)=\int_{M} f(m) d T(m) .
$$

Definition 5.11. Suppose $(\pi, V)$ is a continuous representation of a $\{$ def : distnchar $\}$ Lie group $G$. Every test density $T$ on $G$ defines a continuous linear operator

$$
\pi(T)=\int_{G} \pi(g) d T(g) \in \operatorname{End}(V) .
$$

These operators are typically much "nicer" (in terms of decay properties) than the operators $\pi(g)$, because the integration against $T$ is a kind of smoothing. We say that $\pi$ has a distribution character if the operators $\pi(T)$ are all trace class, and the linear map

$$
\Theta_{\pi}(T)=\operatorname{tr}(\pi(T))
$$

is continuous on test densities. (This requires that $V$ be a space on which there is a reasonable theory of traces of endomorphisms; for our purposes Hilbert spaces will suffice.) In this case the generalized function $\Theta_{\pi}$ is called the distribution character of $\pi$.

Notice that if $\pi$ is finite-dimensional, then $\Theta_{\pi}$ is the ordinary continuous function $\operatorname{tr}(\pi(g))$ on $G$. If $\pi$ is infinite-dimensional and unitary, then the unitary operators $\pi(g)$ are never of trace class, so $\operatorname{tr}(\pi(g))$ is not defined for any $g$. That is why the following theorem of Harish-Chandra is remarkable: it attaches a meaning to $\operatorname{tr}(\pi(g))$ for most $g$.

Theorem 5.12 (Harish-Chandra and others). Suppose $G$ is a real reductive

Lie group, and $(\pi, V)$ is a quasisimple representation of finite length on a Hilbert space $V$.

1. For every test density $T$, the operator $\pi(T)$ is of trace class. The resulting linear functional on test densities is continuous, so that the character

$$
\Theta_{\pi}(T)={ }_{\text {def }} \operatorname{tr}(\pi(T))
$$

is a well-defined generalized function on $G$. 
2. The generalized function $\Theta_{\pi}$ is given by a locally $L^{1}$ function on $G$, which is analytic on the open subset of regular semisimple elements.

3. Suppose $H \subset G$ is a Cartan subgroup. Fix a set $R_{\mathbb{R}}^{+}$of positive roots for the system $R_{\mathbb{R}}$ of real roots of $H$ in $\mathfrak{g}$. Since each real root takes real values on $H$, we can define

$$
H^{+}=\left\{h \in H|| \alpha(h) \mid \geq 1\left(\alpha \in R_{\mathbb{R}}^{+}\right)\right\}
$$

Write $\widetilde{H}$ for the $\rho$ double cover of $H$ (Lemma 5.6), and $D$ for a Weyl denominator function on $\widetilde{H}$ (Definition 5.10). Then the product $D$. $\left.\Theta_{\pi}\right|_{H}$ (an analytic function on the set of regular elements of $\widetilde{H}$ ) is equal on $\widetilde{H}_{\text {reg }}^{+}$to a finite integer combination of characters of $\widetilde{H}$ :

$$
\Theta_{\pi}(\tilde{h})=\left(\sum_{\gamma \in(\widetilde{H})_{1}^{-}} a_{\pi}(\gamma) \gamma(\tilde{h})\right) / D(\tilde{h}) \quad\left(\tilde{h} \in \widetilde{H}_{r e g}^{+}\right) .
$$

The subscript 1 on $(\widetilde{H})_{1}$ (Definition 5.1) is "level 1," meaning that the characters take the value -1 on the central element -1 of the cover. Therefore both the numerator and denominator are "level 1" functions, so the quotient is "level 0 " and defines a function on $H$. The simplest example of this formula is the Weyl character formula for the $2 j+1$-dimensional representation $\pi_{2 j+1}$ of $S O(3)$, which is

$$
\operatorname{tr} \pi_{2 j+1}\left(\begin{array}{ccc}
\cos \theta & \sin \theta & 0 \\
-\sin \theta & \cos \theta & 0 \\
0 & 0 & 1
\end{array}\right)=\frac{\sin \left(\left(j+\frac{1}{2}\right) \theta\right)}{\sin \left(\frac{1}{2} \theta\right)} .
$$

In this formula, the numerator and denominator are well defined only on the double cover of the maximal torus. Nevertheless we like this formula because it is very simple. (It is the formula of Theorem 5.12(3) with numerator and denominator each divided by $2 i$.)

Proof. Part (1) is in [11], pages 243-245. The analyticity in part (2) is the main result of [12]; now that distribution theory is a familiar tool, (2) is a routine "elliptic regularity" argument. But the proof that $\Theta_{\pi}$ actually coincides with integration against this function is much deeper, and was achieved only in [13].

Part (3) is difficult to attribute properly. The fact that the numerator looks locally like a trigonometric polynomial on $\widetilde{H}$ is due to Harish-Chandra, 
and is proved in [12]. Harish-Chandra's arguments, which proceed by solving differential equations, allow also terms with a polynomial dependence on $\widetilde{h}$. That these polynomials are always constant was first proved in [8].

Proving that the coefficients in (3) are actually integers is best accomplished by realizing the numerator as the trace of the action of $\tilde{h}$ on a virtual representation of $\widetilde{H}$. To write down such a virtual representation, fix first of all a Cartan involution $\theta$ on $G$ preserving $H$. Let $\mathfrak{n}^{-} \subset \mathfrak{g}$ be the sum of the negative root spaces for some positive root system $R^{+} \supset R_{\mathbb{R}}^{+}$. Then the Lie algebra cohomology spaces $H^{p}\left(\mathfrak{n}^{-}, V_{K}^{\infty}\right)$ with coefficients in the HarishChandra module of $V$ (Definition 2.14) are finite-dimensional (h, $H \cap K$ )modules. (The finite-dimensionality requires proof; this is provided in [14] and [40].) According to Theorem 2.13, finite-dimensional (h, $H \cap K)$-modules may be identified with representations of $H$. The numerator of the character formula in (3) is the character of the virtual representation of $\widetilde{H}$

$$
\mathbb{C}_{\rho_{\mathrm{abs}}^{-}} \otimes\left(\sum_{p}(-1)^{p} H^{p}\left(\mathfrak{n}^{-}, V_{K}^{\infty}\right)\right) .
$$

Here $\mathbb{C}_{\rho_{\text {abs }}^{-}}$is the space of the defining character $\rho_{\text {abs }}^{-}$of $\widetilde{H}$ (Lemma 5.9), with respect to the positive root system consisting of the roots $R^{-}$in $\mathfrak{n}^{-}$.

The first very general result of this nature is the main theorem of [14]; that result allows one to reduce (3) to the case of a compact Cartan subgroup $H$. A parallel result [40], Theorem 8.1 allows reduction of (3) to the case of a split Cartan subgroup. Applying these two results in succession (in either order!) proves (3).

\section{Langlands classification and the nonunitary dual}

We can now give a precise formulation of the classification of the nonuni$\{$ sec: langlands\} tary dual $\widehat{G}$. This is extremely important, since we are going to explain how to compute the unitary dual as a subset of the nonunitary dual. The most familiar case of the Langlands classification is for compact connected Lie groups: in that case the irreducible representations are in one-to-one correspondence with orbits of the Weyl group on the lattice of characters of a maximal torus. (The correspondence sends an irreducible representation to its set of extremal weights.) We will use a slightly different version of this correspondence, in which an irreducible representation of a compact group corresponds to the (Weyl group orbit) of terms in the numerator of the Weyl character formula. This modified correspondence extends to real reductive 
groups fairly easily and completely. Just as for compact groups, it says (approximately) that the irreducible representations are indexed by orbits of the Weyl groups on characters of (the $\rho$-coverings of) maximal tori. The implementation (approximately) says that a representation corresponds to some character appearing in the numerator of the Weyl character formula (Theorem 5.12) for the representation.

What is needed to make this precise is a way to pick out a "preferred" term in the Weyl character formula. (In the case of compact $G$, all the terms belong to the same Weyl group orbit, so no choice is necessary.) Langlands' idea in the noncompact case was to pick a term with largest growth at infinity as the preferred term. His original version in [24] made a clean and precise connection between growth and the classification; but at the same time lumped together several different representations. Subsequent work (notably [22]) refined this into a precise classification of representations, but at the expense of obscuring slightly the connection with character formulas. Here is a statement. There is a closely related result on page 234 of [30]; but the emphasis there is on reducibility of standard tempered representations, so it is difficult to extract exactly what we want. The best reference we know is [1]; but the formulation here is a bit different. The main change is that we have modified the covering of $H$. This change means that we no longer need to keep track of a choice of positive real roots; the definition of Langlands parameter here is accordingly simpler than in [1].

Theorem 6.1 (Langlands classification; see [1], Chapter 11). Suppose G is the group of real points of a connected complex reductive algebraic group (cf. (3.1)). Then there is a one-to-one correspondence between infinitesimal equivalence classes of irreducible quasisimple representations of $G$ (Definition 2.14) and G-conjugacy classes of triples ("Langlands parameters")

$$
\Gamma=\left(H, \gamma, R_{i \mathbb{R}}^{+}\right),
$$

subject to the following requirements.

1. The group $H$ is a Cartan subgroup of $G$ : the group of real points of a maximal torus of $G(\mathbb{C})$ defined over $\mathbb{R}$.

2. The character $\gamma$ is level one character of the $\rho_{\mathrm{abs}}$ double cover of $H$ (Definition 5.1, Lemma 5.9). Write $d \gamma \in \mathfrak{h}^{*}$ for its differential.

3. The roots $R_{i \mathbb{R}}^{+}$are a positive system for the imaginary roots of $H$ in $\mathfrak{g}$.

4. The weight $d \gamma$ is weakly dominant for $R_{i \mathbb{R}}^{+}$. 
5. If $\alpha^{\vee}$ is real and $\left\langle d \gamma, \alpha^{\vee}\right\rangle=0$, then $\gamma_{\mathfrak{q}}\left(m_{\alpha}\right)=+1$ (Definition 5.7). (Here $\gamma_{\mathfrak{q}}$ is a " $\rho$-shift" of $\gamma$ defined in $(9.3 \mathrm{~g})$ below.)

6. If $\beta$ is simple for $R_{i \mathbb{R}}^{+}$and $\left\langle d \gamma, \beta^{\vee}\right\rangle=0$, then $\beta$ is noncompact.

This one-to-one correspondence may be realized in the following way. Attached to each (equivalence class) of Langlands parameters $\Gamma$ there is a "standard (limit) $\left(\mathfrak{g}_{0}, K\right)$-module" $I(\Gamma)=I_{\text {quo }}(\Gamma)$, which has finite length. This module has a unique irreducible quotient module $J(\Gamma)$; the correspondence is $\Gamma \leftrightarrow J(\Gamma)$. (Alternatively, there is a standard module $I_{\text {sub }}(\Gamma)$ having $J(\Gamma)$ as its unique irreducible submodule. The two standard modules $I_{\mathrm{quo}}(\Gamma)$ and $I_{\text {sub }}(\Gamma)$ have the same composition factors and multiplicities.)

The irreducible $J(\Gamma)$ and the corresponding standard modules all have infinitesimal character $d \gamma \in \mathfrak{h}^{*}$.

Definition 6.2. In the setting of Theorem 6.1, the set of $G$-conjugacy $\{$ def : langlandsparam $\}$ classes of triples $\Gamma=\left(H, \gamma, R_{i \mathbb{R}}^{+}\right)$satisfying conditions (1)-(6) is called the set of Langlands parameters for $G$, and written $\Pi(G)$.

Occasionally we will need some generalizations of the Langlands parameters in Theorem 6.1. Conditions (1)-(3) are essential to make the construction of $I(\Gamma)$ described below; conditions (4)-(6) are imposed so that $I(\Gamma)$ has additional nice properties. We record here some basic facts about these generalizations.

Definition 6.3. In the setting of Theorem 6.1 , a triple $\Gamma=\left(H, \gamma, R_{i \mathbb{R}}^{+}\right)$ $\{$ def : genparam $\}$ satisfying conditions (1)-(3) is called a continued Langlands parameter. The set of equivalence classes of such parameters is written $\Pi_{\text {cont }}(G)$.

Attached to each $\Gamma \in \Pi_{\text {cont }}(G)$ there is a continued standard (virtual) $\left(\mathfrak{g}_{0}, K\right)$-module $I(\Gamma)$ of finite length. (The construction of $I(\Gamma)$ involves some cohomological functors, and in this setting the functors may be nonzero in several different degrees. The virtual representation $I(\Gamma)$ is the alternating sum of these functors.)

If in addition $\Gamma$ satisfies condition (4), then $\Gamma$ is called a weak Langlands parameter. The set of weak parameters is written $\Pi_{\text {weak }}(G)$.

Under this hypothesis there is a vanishing theorem, and one can construct weak standard $\left(\mathfrak{g}_{0}, K\right)$-modules $I_{\mathrm{quo}}(\Gamma)$ and $I_{\mathrm{sub}}(\Gamma)$. A weak standard module is always a direct sum of a finite number (possibly zero) of standard modules. For any weak Langlands parameter $\Gamma$, we can therefore define the Langlands quotient representation $J(\Gamma)$ to be the corresponding direct sum of irreducible representations; this is the cosocle of $I_{\text {quo }}(\Gamma)$, or equivalently the socle of $I_{\text {sub }}(\Gamma)$. 
If $\Gamma$ does not satisfy condition (4), then we do not know any reasonable way to define $J(\Gamma)$.

In order to understand where the restrictions on $\Gamma$ come from, we recall Langlands' construction of $I(\Gamma)$. Write $M A$ for the Langlands decomposition of the centralizer of $A$ in $G$. (This centralizer is the group of real points of a connected complex reductive algebraic group, but the factors $M$ and $A$ need not be. Our chosen perspective would therefore prefer not to make this factorization, but it is traditional and some of us are nothing if not hidebound.) The compact group $T$ is a compact Cartan subgroup of $M$, and the parameters

$$
\Lambda=\left(T,\left.\gamma\right|_{T}, R_{i \mathbb{R}}^{+}\right)
$$

are (according to conditions $1-4$ of Theorem 6.1) Harish-Chandra parameters for a limit of discrete series representation $D(\Lambda) \in \widehat{M}$. A construction of $D(\Lambda)$ by Zuckerman's cohomological induction functors is a special case of Theorem 9.4. If "weakly dominant" is strengthened to "strictly dominant" in condition 4 , we get a discrete series representation. If condition 4 is dropped, then the vanishing-except-in-one-degree statement of Theorem 9.4 can fail, so $D(\Lambda)$ becomes only a virtual representation (see Definition $6.3)$.

For a weak parameter $\Gamma$ (that is, assuming condition 4) condition 6 is exactly what is required to make $D(\Lambda)$ nonzero. (The fact that $D(\Lambda)=0$ if $d \lambda$ vanishes on a compact simple coroot is the simpler of the eponymous character identities of [33].) So condition 6 is needed to get a nonzero standard representation.

Now write

$$
\nu=\left.\gamma\right|_{A} \in \widehat{A}
$$

for the (possibly nonunitary) character of $A$ defined by $\Gamma$. (The character $2 \rho_{i \mathbb{R}}$ (sum of the positive imaginary roots) is trivial on $A$. According to Lemma $5.9(3)$, it follows that the level one character $\gamma \in(\widetilde{H})_{1}$ may be regarded as a pair $(\lambda, \nu)$, with $\lambda \in(\widetilde{T})_{1}$ and $\nu \in \widehat{A}$.)

Choose a parabolic subgroup $P=M A N$ of $G$ making (the real part of) $\nu$ weakly dominant for the weights of $\mathfrak{a}$ in $\mathfrak{n}$. Then the standard representation of Theorem 6.1 may be realized as

$$
I_{\mathrm{quo}}(\Gamma)=\operatorname{Ind}_{P}^{G} D(\Lambda) \otimes \nu \otimes 1
$$

(The dominance requirement does not specify $P$ uniquely, but all choices give isomorphic standard representations. Replacing $P$ by the opposite parabolic 
subgroup $P^{\text {op }}$ replaces $I_{\text {quo }}(\Gamma)$ by $I_{\text {sub }}(\Gamma)$.) If condition 5 fails in Theorem 6.1, then there is a real coroot $\alpha^{\vee}$ with $\left\langle d \gamma, \alpha^{\vee}\right\rangle=0$; equivalently, $\left\langle\nu, \alpha^{\vee}\right\rangle=$ 0 . Possibly after modifying $P$, this coroot defines a parabolic subgroup

$$
P_{\alpha}=M_{\alpha} A_{\alpha} N_{\alpha} \supset P .
$$

Induction by stages realizes the standard representation $I(\Gamma)$ as

$$
I_{\text {quo }}(\Gamma)=\left.\operatorname{Ind}_{P_{\alpha}}^{G}\left[\operatorname{Ind}_{M\left(A \cap M_{\alpha}\right)\left(N \cap M_{\alpha}\right)} D(\Lambda) \otimes 1 \otimes 1\right] \otimes \nu\right|_{A_{\alpha}} \otimes 1 .
$$

The fact that the inner induction involves the trivial character 1 of $A \cap$ $M_{\alpha}$ is precisely the condition $\left\langle\nu, \alpha^{\vee}\right\rangle=0$. The inner induction to $M_{\alpha}$ is therefore unitary induction. We claim that when condition 5 above fails (that is, when $\gamma_{\mathfrak{q}}\left(m_{\alpha}\right)=-1$ ) then this induced representation may be written (using the first character identity of [33]) as a sum of one or two standard representations attached to the (more compact) Cartan subgroup obtained from $H$ by Cayley transform through the real root $\alpha$. In case $G=S L(2)$, this claim is just the familiar fact that the nonspherical principal series representation with continuous parameter zero is a sum of two limits of discrete series. More generally, when the Cartan subgroup $H$ is split, the group $M_{\alpha}$ is locally isomorphic to $S L(2, \mathbb{R})$, and the claim again reduces to $S L(2)$. In general, one can realize the standard representation $I(\Gamma)$ by cohomological induction from a split Levi subgroup of $G$ (see Section 9) and in this way reduce to the case when $H$ is split.

The conclusion is that when condition 5 fails, the standard representation $I(\Gamma)$ may be realized as a direct sum of one or two representations attached to weak parameters $\Gamma^{\prime}$ on a Cartan subgroup with strictly larger compact part. That is, imposing condition 5 seeks to make a canonical realization of $I(\Gamma)$ : one attached to the most compact Cartan subgroup possible. These remarks do not prove that the realization is canonical, but this turns out to be the case.

This discussion of the realization of $I(\Gamma)$ suggests the following definitions; we will use them constantly in analyzing unitary representations.

Definition 6.5. Suppose $G$ is the group of real points of a connected complex reductive algebraic group (cf. (3.1)). A discrete Langlands parameter is a triple $\Lambda=\left(T, \lambda, R_{i \mathbb{R}}^{+}\right)$, subject to the following conditions.

1. The group $T$ is the maximal compact subgroup of a Cartan subgroup $H$ of $G$ (Proposition 4.2).

2. The character $\lambda$ is a level one character of the $\rho_{i \mathbb{R}}$ double cover of $T$ (Definition 5.1, Lemma 5.9). (Write $d \lambda \in \mathfrak{t}^{*}$ for the differential of $\lambda$.) 
3. The roots $R_{i \mathbb{R}}^{+}$are positive roots for the imaginary roots of $H$ in $\mathfrak{g}$.

4. The weight $d \lambda$ is weakly dominant for $R_{i \mathbb{R}}^{+}$.

5. If $\beta$ is a simple root of $R_{i \mathbb{R}}^{+}$and $\left\langle d \lambda, \beta^{\vee}\right\rangle=0$, then $\beta$ is noncompact.

Two discrete Langlands parameters are called equivalent if they are conjugate by $G$. The set of equivalence classes of discrete Langlands parameters is written

$$
\Pi_{\text {disc }}(G) .
$$

Alternatively, one can define discrete Langlands parameters up to $K(\mathbb{C})$ conjugacy using the $\theta$-fixed subgroup $T(\mathbb{C})$ of a $\theta$-stable maximal torus $H(\mathbb{C})$. The $K(\mathbb{C})$-equivalence classes in this alternate definition are in one-to-correspondence with the $G$-equivalence classes above, the equivalence being implemented by choice of a $\theta$-stable representative in the $G$-class.

A discrete parameter $\Lambda$ is called final if in addition it satisfies

6. If $\alpha^{\vee}$ is real, then $\lambda_{\mathfrak{q}}\left(m_{\alpha}\right)=1($ see $(9.3 \mathrm{~g}))$.

We write $\Pi_{\text {fin,disc }}(G)$ for the set of final discrete parameters. Suppose now that $\Lambda=\left(T, \lambda, R_{i \mathbb{R}}^{+}\right)$is a discrete Langlands parameter for $G$. A continuous parameter for $\Lambda$ is a pair $(A, \nu)$, subject to the following conditions:

1. The group $A$ is the vector part of a real Cartan subgroup $H$ with maximal compact $T$ (Proposition 4.2).

2. $\nu$ is a (possibly nonunitary) character of $A$; equivalently, $\nu \in \widehat{A} \simeq \mathfrak{a}^{*}$.

3. If $\alpha^{\vee}$ is real and $\left\langle\nu, \alpha^{\vee}\right\rangle=0$, then $\lambda_{\mathfrak{q}}\left(m_{\alpha}\right)=+1((9.3 \mathrm{~g}))$.

Two such continuous parameters are called equivalent if they are conjugate by the stabilizer $N(\Lambda)$ of $\Lambda$ in $G(\mathbb{R})$.

Proposition 6.6. Fix a discrete Langlands parameter $\Lambda=\left(T, \lambda, R_{i \mathbb{R}}^{+}\right)$, and \{prop:LCshape $\}$ a real Cartan subgroup $H(\mathbb{R})=T A$. Define

$$
W^{\Lambda}=\{w \in W(G, H) \mid w \cdot \Lambda=\Lambda\},
$$

the stabilizer of $\Lambda$ in the real Weyl group (Definition 5.7). 
1. Introduce a $\mathbb{Z} / 2 \mathbb{Z}$-grading on the real coroots $R_{\mathbb{R}}^{\vee}$ by (notation $(9.3 \mathrm{~g}$ ))

$$
\alpha^{\vee} \text { is odd } \Longleftrightarrow \Lambda_{\mathfrak{q}}\left(m_{\alpha}\right)=-1 \text {. }
$$

Then the set of equivalence classes of continuous parameters for $\Lambda$ is

$$
\left(\mathfrak{a}^{*}-\bigcup_{\alpha^{\vee}} \bigcup_{\text {real odd }} \operatorname{ker}\left(\alpha^{\vee}\right)\right) / W^{\lambda} .
$$

2. Write $\mathcal{L}(\Lambda)$ for the set of lowest $K$-types ([38], Definition 5.4.18) of the standard representations $I(\Lambda, \nu)$ (with $\nu$ a continuous parameter for $\Lambda)$. These $K$-types all have multiplicity one in $I(\Lambda, \nu)$, and they all appear in the Langlands quotients $J(\Lambda, \nu)$.

3. The element $0 \in \widehat{A}$ is a continuous parameter for $\Lambda$ if and only if $\Lambda$ is final. In this case $\mathcal{L}(\Lambda)$ consists of exactly one irreducible representation $\mu(\Lambda) \in \widehat{K}$.

4. The Langlands quotient $J(\Lambda, \nu)$ is tempered if and only if $\nu \in \widehat{A}_{u} \simeq i \mathfrak{a}_{0}^{*}$ is a unitary character. In this case (and still assuming $\nu$ is a continuous parameter for $\Lambda$ ) the standard representation $I(\Lambda, \nu)$ is unitary and irreducible.

5. The infinitesimal character of the Langlands quotient $J(\Lambda, \nu)$ is real ([38], Definition 5.4.11) if and only if $\nu \in \mathfrak{a}_{0}^{*}$ is a real-valued character.

6. The standard representation $I(\Lambda, \nu)$ is reducible only if either

a) there is a real root $\alpha$ such that

$$
\left\langle\nu, \alpha^{\vee}\right\rangle \in \mathbb{Z} \backslash\{0\}, \quad \Lambda_{\mathfrak{q}}\left(m_{\alpha}\right)=(-1)^{\left\langle\nu, \alpha^{\vee}\right\rangle+1} ;
$$

or

b) there is a complex root $\delta$ such that $\left\langle(d \lambda, \nu), \delta^{\vee}\right\rangle \in \mathbb{Z}$, and

$$
\left\langle\nu, \delta^{\vee}\right\rangle>\left|\left\langle d \lambda, \delta^{\vee}\right\rangle\right| \text {. }
$$

Proof. The description of continuous parameters in (1) is just a reformulation of Theorem 6.1. The statement about lowest $K$-types in (2) largely comes from Theorem 6.5.12 in [38], although some translation of language is needed to get to this formulation. The first assertion in (3) is immediate from (1), and the rest can be deduced from Theorem 6.5.12 in [38]. (This is the central idea in [44].) 
For (4), that the Langlands quotient is tempered if and only if the continuous parameter is unitary is part of Langlands' original result. The fact that the standard representation is irreducible (under the assumption that the unitary continuous parameter does not vanish on any real odd root) is a very special case of (6). Part (5) is immediate from (and in fact motivates) the definition of real infinitesimal character. Finally (6) is the main theorem of $[36]$.

The point of the proposition is to describe the shape of the nonunitary dual of $G$. It is a countable union (over the set of equivalence classes of discrete Langlands parameters $\Lambda$ ) of connected pieces. Each connected piece is a complex vector space $\mathfrak{a}^{*}$, minus a finite collection of hyperplanes through the origin (defined by the odd real coroots), modulo the action of a finite group $W^{\Lambda}$. (At the missing hyperplanes, the standard representations can be rewritten as a direct sum of one or two standard representations attached to a more compact Cartan subgroup. So the "missing" representations still exist; they are just attached to a different discrete Langlands parameter, and may be reducible.)

The complex vector space $\mathfrak{a}^{*}$ is naturally defined over $\mathbb{R}$; the real subspace $\mathfrak{a}_{0}^{*}$ corresponds to characters of $A$ taking positive real values. Indeed it is defined over the integers: we can take as integer points

$$
\left(X^{*}\right)^{-\theta} \simeq(-\theta) \text {-fixed algebraic characters of } H \text { restricted to } A \text {. }
$$

\section{Second introduction: the shape of the unitary dual}

We now have enough machinery in place to describe the kind of answer we are going to find for describing the unitary dual of a real reductive group $G$ (cf. (3.1)).

Theorem 7.1 (Knapp-Zuckerman; see [19], Chapter 16). Suppose $\Gamma=$ $(\Lambda, \nu)$ is a Langlands parameter for an irreducible quasisimple representation $J(\Gamma)$ for the real reductive group $G$ (Theorem 6.1), corresponding to the Cartan subgroup $H=T A$. Define

$$
W^{\Lambda} \subset W(G, H)
$$

as in Proposition 6.6.

Write the continuous parameter $\nu$ as

$$
\nu=\nu_{\mathrm{re}}+i \nu_{\mathrm{im}} \quad\left(\nu_{\mathrm{re}}, \nu_{\mathrm{im}} \in \mathfrak{a}_{0}^{*}\right) .
$$


Define

$$
L_{\mathrm{im}}=G^{\nu_{\mathrm{im}}},
$$

the real Levi subgroup of $G$ corresponding to coroots vanishing on the imaginary part of $\nu$.

1. The irreducible representation $J(\Gamma)$ admits an invariant Hermitian form if and only if there is a $w \in W^{\Lambda}$ such that

$$
w \cdot \nu=-\bar{\nu}
$$

equivalently

$$
w \cdot \nu_{\mathrm{im}}=\nu_{\mathrm{im}}, \quad w \cdot \nu_{\mathrm{re}}=-\nu_{\mathrm{re}} .
$$

The first condition here is equivalent to $w \in W_{L_{\mathrm{im}}}^{\Lambda}$; so we conclude that $J(\Gamma)$ admits an invariant Hermitian form if and only if the irreducible $J_{L_{\mathrm{im}}}(\Gamma)$ for $L_{\mathrm{im}}$ admits an invariant Hermitian form.

2. The irreducible representation $J(\Gamma)$ is irreducibly induced from the representation $J_{L_{\mathrm{im}}}(\Gamma)$ on $L_{\mathrm{im}}$. In the presence of invariant Hermitian forms, this induction is unitary, so $J(\Gamma)$ is unitary if and only if $J_{L_{\mathrm{im}}}(\Gamma)$ is unitary. This in turn is the case if and only if $J_{L_{\mathrm{im}}}\left(\left(\Lambda, \nu_{\mathrm{im}}\right)\right.$ is unitary.

We will explain thoroughly and precisely what an "invariant Hermitian form" is beginning in Section 8 (see especially (10.2)). At that point we will explain some of the proof of this theorem.

This theorem exhibits every unitary representation as unitarily induced from a unitary representation with real continuous parameter; equivalently, with "real infinitesimal character" (Definition 5.5), in a precise and simple way. The question we need to address is classification of irreducible unitary representations with real infinitesimal character; that is, determining which representations with real continuous parameter are unitary.

Corollary 7.2. Fix a discrete Langlands parameter $\Lambda$, attached to a Cartan subgroup $H=T A$, and $W^{\Lambda}$ the stabilizer in the real Weyl group (Proposition 6.6). The real continuous parameters attached to $\Lambda$ are

$$
\left(\mathfrak{a}_{0}^{*}-\bigcup_{\alpha^{\vee}} \bigcup_{\text {real odd }} \operatorname{ker}\left(\alpha^{\vee}\right)\right) / W^{\Lambda}
$$

(Proposition 6.6). For each $w \in W^{\Lambda}$, consider the -1 eigenspace

$$
\mathfrak{a}_{w}^{*}={ }_{\text {def }}\left\{\nu \in \mathfrak{a}^{*} \mid w \cdot \nu=-\nu\right\},
$$

and its real form $\mathfrak{a}_{w, 0}^{*}$. 
1. The Hermitian representations $J(\Lambda, \nu)$ with real continuous parameter are precisely those with $\nu \in \mathfrak{a}_{w, 0}^{*}$, for some $w \in W^{\Lambda}$.

2. Fix $w \in W^{\Lambda}$. Each of the hyperplanes of possible reducibility described in Proposition 6.6 meets $\mathfrak{a}_{w, 0}^{*}$ in a hyperplane, or not at all. In this way we get a locally finite hyperplane arrangement in $\mathfrak{a}_{w, 0}^{*}$, partitioning this vector space into a locally finite collection of facets; each facet is characterized by various equalities

$$
\left\langle(d \lambda, \nu), \delta^{\vee}\right\rangle=m_{\delta}
$$

and inequalities

$$
\left\langle(d \lambda, \nu),\left(\delta^{\prime}\right)^{\vee}\right\rangle<\ell_{\delta^{\prime}}
$$

for roots $\delta, \delta^{\prime}$ and integers $m_{\delta}, \ell_{\delta^{\prime}}$.

3. The signature of the Hermitian form on $J(\Lambda, \nu)$ (for $\nu \in \mathfrak{a}_{w, 0}^{*}$ ) is constant on each of the facets described in (2).

4. For each $w \in W^{\Lambda}$ there is a compact rational polyhedron $P_{w} \subset \mathfrak{a}_{0, w}^{*}$ (a finite union of the facets of (2)) so that if $\nu$ is a continuous real parameter attached to $\Lambda$, then $J(\Lambda, \nu)$ is unitary if and only if $\nu$ belongs to some $P_{w}$.

Again, this corollary describes only what was known about the structure of the unitary dual in the 1980s.

The main result of this paper is an algorithm to calculate the signature of the invariant Hermitian form on $J(\Lambda, \nu)$ for $\nu$ belonging to any specified facet of one of the vector spaces $\mathfrak{a}_{0, w}^{*}$. (Exactly what is meant by "calculate the signature" will be explained in Section 15.) In particular, this algorithm decides whether or not the facet consists of unitary representations. As was explained already in [43], there is some predictable repetition in these calculations; so doing a finite number of them is enough to determine the unitary dual of $G$.

\section{Hermitian forms on $(\mathfrak{h}, L(\mathbb{C}))$-modules}

We turn now to the study of invariant forms on representations. The work $\{$ sec:forms that we do depends fundamentally on generalizing and modifying the notion of "invariant form." We will therefore look very carefully and slowly at this notion. A convenient setting (allowing us to consider both group and Lie algebra representations) is that of a "pair." 
Definition 8.1 ([21], Definition 1.64). A pair is $(\mathfrak{h}, L(\mathbb{C})$ ), together with

$$
\operatorname{Ad}: L(\mathbb{C}) \rightarrow \operatorname{Aut}(\mathfrak{h}), \quad i: \mathfrak{l} \hookrightarrow \mathfrak{h},
$$

subject to

a) $\mathfrak{h}$ is a complex Lie algebra

b) $L(\mathbb{C})$ is a complex algebraic group, with (complex) Lie algebra $\mathfrak{l}$

c) $i$ is an inclusion of complex Lie algebras

d) Ad is an algebraic action by automorphisms of $\mathfrak{h}$, extending the adjoint action of $L(\mathbb{C})$ on $\mathfrak{l}$, and

e) the differential of Ad is the adjoint action (by Lie bracket) of $\mathfrak{l}$ on $\mathfrak{h}$.

A representation of the pair $(\mathfrak{h}, L(\mathbb{C}))($ cf. Definition 2.10$)$ is a complex vector space $V$ endowed with a complex-linear representation of the Lie algebra $\mathfrak{h}$ and an algebraic representation of the algebraic group $L(\mathbb{C})$, subject to the requirements

a) the differential of the action of $L(\mathbb{C})$ (a representation of $\mathfrak{l}$ ) is equal to the composition of $i$ with the action of $\mathfrak{h}$; and

b)

$$
\ell \cdot(X \cdot v)=(\operatorname{Ad}(\ell) X) \cdot(\ell \cdot v), \quad(\ell \in L(\mathbb{C}), X \in \mathfrak{h}, v \in V) .
$$

A real structure on the pair consists of two maps

$$
\sigma: \mathfrak{h} \rightarrow \mathfrak{h}, \quad \sigma: L(\mathbb{C}) \rightarrow L(\mathbb{C})
$$

both "conjugate linear" in the following sense. First, the map on $\mathfrak{h}$ is a conjugate linear Lie algebra homomorphism:

$\sigma(z X)=\bar{z} \sigma(X), \quad \sigma([X, Y])=[\sigma(X), \sigma(Y)] \quad(z \in \mathbb{C}, X, Y \in \mathfrak{h})$.

These conditions are all that is needed to define invariant sesquilinear forms on representations; but to make sense of invariant Hermitian forms, we must assume also that $\sigma$ is a complex structure on $\mathfrak{h}$, meaning that

$$
\sigma^{2}=\mathrm{Id}
$$


Second, the map on $L(\mathbb{C})$

$$
\sigma: L(\mathbb{C}) \rightarrow L(\mathbb{C})
$$

is a group homomorphism so that the corresponding map on functions

$$
\left(\sigma^{*} f\right)(\ell)=\overline{f(\sigma(\ell))}
$$

preserves regular functions on $L(\mathbb{C})$ :

$$
\sigma^{*}: R(L(\mathbb{C})) \rightarrow \overline{R(L(\mathbb{C}))}
$$

(By construction $\sigma^{*}$ is a conjugate-linear homomorphism of algebras.) Again this assumption is all that is needed to define invariant sesquilinear forms, but to get invariant Hermitian forms we must assume also that $\sigma$ is a real form of the complex algebraic group $L(\mathbb{C})$; that is, that

$$
\sigma^{2}=\mathrm{Id}
$$

We will be interested most of all in the pair $(\mathfrak{g}, K(\mathbb{C}))$ coming from a real reductive algebraic group $G$, described in Theorem 3.4. We include more general pairs for the same reason that they appear in [21]: as a tool in the construction of Harish-Chandra modules for $G$.

Suppose $V$ is a complex vector space (or a representation of a complex Lie algebra $\mathfrak{h}$, or an algebraic representation of an algebraic group $L(\mathbb{C})$, or an $(\mathfrak{h}, L(\mathbb{C}))$-module (Definition 8.1)). Recall the Hermitian dual of $V$

$$
V^{h}=\{\xi: V \rightarrow \mathbb{C} \mid \xi(u+v)=\xi(u)+\xi(v), \xi(z \cdot v)=\bar{z} \cdot v\}
$$

(We are going to modify this definition a little in the presence of an algebraic group representation; see (8.2f) below.) It is clear that the Hermitian dual of $V$ consists of complex conjugates of the linear functionals on $V$. Taking Hermitian dual is a contravariant functor: if $T \in \operatorname{Hom}(V, W)$, then the Hermitian transpose of $T$ is

$$
T^{h} \in \operatorname{Hom}\left(W^{h}, V^{h}\right), \quad T^{h}(\xi)(v)=\xi(T(v)) \quad\left(v \in V, \xi \in W^{h}\right) .
$$

What makes everything complicated and interesting is that the Hermitian transpose operation is not complex linear:

$$
(S \circ T)^{h}=T^{h} \circ S^{h}, \quad(z T)^{h}=\bar{z}\left(T^{h}\right) .
$$


To see why this is a complication, suppose that $\rho: \mathfrak{h} \rightarrow \operatorname{End}(V)$ is a representation of the complex Lie algebra $\mathfrak{h}$. This means that $\rho$ is a complexlinear Lie algebra homomorphism. If we try to take Hermitian transpose, we find

$$
\rho^{h}: \mathfrak{h} \rightarrow \operatorname{End}\left(V^{h}\right), \quad \rho^{h}(X)=-[\rho(X)]^{h}
$$

is additive and respects the Lie bracket; but $\rho^{h}(z X)=\bar{z} \rho^{h}(X)$, so $\rho^{h}$ is not complex linear.

There is a parallel problem in case $V$ carries an algebraic representation $\pi$ of an algebraic group $L(\mathbb{C})$. The group homomorphism $\pi: L(\mathbb{C}) \rightarrow G L(V)$ is assumed to be algebraic; this means in particular that every matrix coefficient of the homomorphism is an algebraic function on $L(\mathbb{C})$. We can get a group homomorphism

$$
\pi^{h}: L(\mathbb{C}) \rightarrow G L\left(V^{h}\right), \quad \pi^{h}(k)=\left[\pi\left(k^{-1}\right)\right]^{h} .
$$

But the matrix coefficients of $\pi^{h}$ include complex conjugates of the matrix coefficients of $\pi$. Since the complex conjugates of nonconstant algebraic functions are not algebraic, $\pi^{h}$ is almost never an algebraic representation.

There is a smaller technical problem here. Part of what it means for $V$ to be an algebraic representation of $L(\mathbb{C})$ is that the action is locally finite:

$$
\operatorname{dim}\langle\pi(L(\mathbb{C})) v\rangle<\infty \quad(v \in V) .
$$

This condition is not inherited by the Hermitian dual as defined in (8.2a) above. Whenever we have in mind an algebraic representation of $L(\mathbb{C})$ on $V$, it is therefore convenient to modify that definition to the smaller space

$$
\begin{gathered}
V^{h}=\{\xi: V \rightarrow \mathbb{C} \mid \xi(u+v)=\xi(u)+\xi(v), \xi(z \cdot v)=\bar{z} \cdot v \\
\left.\operatorname{dim}\left\langle\pi^{h}(L(\mathbb{C})) \xi\right\rangle<\infty\right\}
\end{gathered}
$$

It would be more natural to retain the definition (8.2a) for $V^{h}$, and to denote this subspace by $V_{L(\mathbb{C})}^{h}$; but for the purposes of this paper, imposing $L(\mathbb{C})$-finiteness silently is more convenient.

We can now discuss forms. Recall that if $V$ and $W$ are complex vector spaces, a sesquilinear pairing between $V$ and $W$ is

$$
\langle,\rangle: V \times W \rightarrow \mathbb{C}, \quad \text { linear in } V, \text { conjugate linear in } W .
$$

We write

$$
\operatorname{Sesq}(V, W)=\{\text { sesquilinear pairings between } V \text { and } W\} \text {. }
$$

A sesquilinear pairing on one vector space $V$ is called Hermitian if it satisfies

$$
\left\langle v, v^{\prime}\right\rangle=\overline{\left\langle v^{\prime}, v\right\rangle} \quad\left(v, v^{\prime} \in V\right) .
$$


Lemma 8.4. Suppose $V$ and $W$ are complex vector spaces.

1. Sesquilinear pairings between $V$ and $W$ may be naturally identified with linear maps from $V$ to the Hermitian dual $W^{h}$ (cf. (8.2a)):

$$
\operatorname{Hom}\left(V, W^{h}\right) \simeq \operatorname{Sesq}(V, W), \quad T \leftrightarrow\langle v, w\rangle_{T}={ }_{\operatorname{def}}(T v)(w) .
$$

2. Complex conjugation defines a (conjugate linear) isomorphism

$$
\operatorname{Sesq}(V, W) \simeq \operatorname{Sesq}(W, V) .
$$

The corresponding (conjugate linear) isomorphism on linear maps is

$$
\operatorname{Hom}\left(V, W^{h}\right) \simeq \operatorname{Hom}\left(W, V^{h}\right), \quad T \mapsto T^{h} .
$$

3. A sesquilinear form $\langle,\rangle_{T}$ on $V$ is Hermitian if and only if $T^{h}=T$.

The statements of the lemma are just versions of the standard dictionary between bilinear forms and linear maps, and the proofs are short and straightforward. In (2), the Hermitian transpose $T^{h}$ was defined as a map from $\left(W^{h}\right)^{h}$ to $V^{h}$. But there is a natural inclusion $W \hookrightarrow\left(W^{h}\right)^{h}$, and it is the corresponding restriction of $T^{h}$ that is intended in (2).

We fix now a pair $(\mathfrak{h}, L(\mathbb{C}))$ with a real structure $\sigma$ (Definition 8.1); we wish to define the $\sigma$-Hermitian dual

$$
V^{h, \sigma}
$$

of an $(\mathfrak{h}, L(\mathbb{C}))$-module $V$. The space is just the space $V^{h}$ defined in (8.2f), of complex conjugates of $L(\mathbb{C})$-finite linear functionals on $V$. To define the Lie algebra representation on $V^{h, \sigma}$, we write $\rho$ for the complex Lie algebra representation on $V$ :

$$
\rho: \mathfrak{h} \rightarrow \operatorname{End}(V) .
$$

In (8.2), we have seen how to define a (complex conjugate linear) Lie algebra representation

$$
\rho^{h}: \mathfrak{h} \rightarrow \operatorname{End}\left(V^{h}\right)
$$

(That this representation preserves the subspace of $L(\mathbb{C})$-finite vectors is an elementary exercise.) In order to get a complex linear Lie algebra representation, we simply compose with the conjugate linear Lie algebra homomorphism $\sigma$, defining the $\sigma$-Hermitian dual Lie algebra representation

$$
\rho^{h, \sigma}: \mathfrak{h} \rightarrow \operatorname{End}\left(V^{h}\right), \quad \rho^{h, \sigma}(X)=-[\rho(\sigma(X))]^{h} .
$$


The construction of the Hermitian dual of the algebraic group representation

$$
\pi: L(\mathbb{C}) \rightarrow G L(V)
$$

is exactly parallel. We have introduced in (8.2) a (nonalgebraic) group representation

$$
\pi^{h}: L(\mathbb{C}) \rightarrow G L\left(V^{h}\right),
$$

which is locally finite on $V^{h}$ (because of the redefinition in (8.2f)). The matrix coefficients of $\pi^{h}$ are complex conjugates of matrix coefficients of $\rho$ (composed with the inversion map on $L(\mathbb{C})$ ), so they are not algebraic. In order to make them algebraic, we need to compose them with our fixed conjugate linear algebraic group homomorphism

$$
\sigma: L(\mathbb{C}) \rightarrow L(\mathbb{C})
$$

The $\sigma$-Hermitian dual representation is

$$
\pi^{h, \sigma}: L(\mathbb{C}) \rightarrow G L\left(V^{h}\right), \quad \pi^{h, \sigma}(k)=\left[\pi\left(\sigma\left(k^{-1}\right)\right]^{h} .\right.
$$

$\{$ eq: dualrep $\}$

This is an algebraic representation of $L(\mathbb{C})$. (The hypothesis on $\sigma$ makes some nice matrix coefficients of $\pi^{h, \sigma}$ into regular functions. The restriction to $L(\mathbb{C})$-finite vectors provides the remaining formal properties making an algebraic representation.)

Of course we can apply this process twice, obtaining a $\sigma$-Hermitian double dual representation on $\left(V^{h, \sigma}\right)^{h, \sigma}$. The representation on $\left(V^{h, \sigma}\right)^{h, \sigma}$ preserves the subspace $V$ (see the remarks after Lemma 8.4) and acts there by the original representation twisted by $\sigma^{2}$. Because of our assumption in Definition 8.1 that $\sigma^{2}=\mathrm{Id}$, it follows that the inclusion $V \subset\left(V^{h, \sigma}\right)^{h, \sigma}$ is an inclusion of $(\mathfrak{h}, L(\mathbb{C}))$-modules.

We define $\sigma$-invariant sesquilinear pairings between $(\mathfrak{h}, L(\mathbb{C}))$-modules $V$ and $W$ : in addition to the sesquilinearity condition in (8.3), we require

$$
\begin{aligned}
\langle X \cdot v, w\rangle & =\langle v,-\sigma(X) \cdot w\rangle, \quad(X \in \mathfrak{h}, \ell \in L(\mathbb{C}), v \in V, w \in W) \\
\langle\ell \cdot v, w\rangle & =\left\langle v, \sigma\left(\ell^{-1}\right) \cdot w\right\rangle \quad(\ell \in L(\mathbb{C}), v \in V, w \in W) .
\end{aligned}
$$

The dictionary in Lemma 8.4 extends immediately to

$$
\operatorname{Sesq}_{\mathfrak{h}, L(\mathbb{C})}^{\sigma}(V, W) \simeq \operatorname{Hom}_{\mathfrak{h}, L(\mathbb{C})}\left(V, W^{h, \sigma}\right)
$$

(Since $V$ is locally $L(\mathbb{C})$-finite, an intertwining operator necessarily takes values in $L(\mathbb{C})$-finite vectors; this uses our assumed surjectivity of $\sigma$.)

We can now define invariant Hermitian forms in the most general way relevant to this paper. 
Definition 8.6. Suppose $(\mathfrak{h}, L(\mathbb{C}))$ is a pair with a real structure $\sigma$ $\{$ def : invherm $\}$ (Definition 8.1), and $V$ is an $(\mathfrak{h}, L(\mathbb{C})$ )-module. A $\sigma$-invariant Hermitian form on $V$ is a Hermitian pairing $\langle$,$\rangle on V$, satisfying

$$
\langle X \cdot v, w\rangle=\langle v,-\sigma(X) \cdot w\rangle \quad(X \in \mathfrak{h}, v, w \in V),
$$

and

$$
\langle\ell \cdot v, w\rangle=\left\langle v, \sigma\left(\ell^{-1}\right) \cdot w\right\rangle \quad(\ell \in L(\mathbb{C}), v, w \in V) .
$$

Such a form may be identified with an intertwining operator

$$
T \in \operatorname{Hom}_{\mathfrak{h}, L(\mathbb{C})}\left(V, V^{h, \sigma}\right)
$$

satisfying the Hermitian condition

$$
T^{h}=T \text {. }
$$

Many examples of pairs arise from a real Lie group $H$ with a compact subgroup $L$. In this case the Lie algebra $\mathfrak{h}$ is the complexification of $\mathfrak{h}_{0}=$ $\operatorname{Lie}(H)$, so we have a complex structure $\sigma_{0}$ on $\mathfrak{h}$ :

$$
\sigma_{0}(X+i Y)=X-i Y \quad\left(X, Y \in \mathfrak{h}_{0}\right) .
$$

At the same time $L(\mathbb{C})$ is the complexification of $L$, and this complexification construction defines the compact real form

$$
\sigma_{0}: L(\mathbb{C}) \rightarrow L(\mathbb{C}), \quad L\left(\mathbb{R}, \sigma_{0}\right)=L
$$

(Theorem 2.17). These two maps $\sigma_{0}$ are a natural real structure for the pair $(\mathfrak{h}, L(\mathbb{C}))$. In this setting, a $\sigma_{0}$-invariant sesquilinear pairing $\langle$,$\rangle between$ $(\mathfrak{h}, L(\mathbb{C}))$-modules $V$ and $W$ may be characterized by the simple requirements

$$
\begin{array}{ll}
\langle X \cdot v, w\rangle=\langle v,-X \cdot w\rangle, \quad & \langle k \cdot v, w\rangle=\left\langle v, k^{-1} \cdot w\right\rangle \\
& \left(X \in \mathfrak{h}_{0}, k \in L, v \in V, w \in W\right) .
\end{array}
$$

The reason we did not simply present this as the definition of an invariant form is that it obscures the possibility of generalization by changing $\sigma_{0}$, which will be crucial for us.

Suppose now that $(\mathfrak{h}, L(\mathbb{C}))$ is a pair with $L(\mathbb{C})$ reductive. In this setting, if $V$ is an $(\mathfrak{h}, L(\mathbb{C}))$-module,

$$
V=\sum_{\mu \in \widehat{L(\mathbb{C})}} V(\mu),
$$


with $V(\mu)$ the largest sum of copies of $\mu$ in $V$. It will be useful to refine this further. So fix a model $E_{\mu}$ for each irreducible representation of $L(\mathbb{C})$. Define

$$
V^{\mu}=\operatorname{Hom}_{L(\mathbb{C})}\left(E_{\mu}, V\right) .
$$

Then there is a natural isomorphism

$$
V(\mu) \simeq V^{\mu} \otimes E_{\mu}, \quad T \otimes e \mapsto T(e) .
$$

The vector space $V^{\mu}$ is called the multiplicity space of $\mu$ in $V$, because of the obvious relation

$$
\operatorname{dim} V^{\mu}=\text { multiplicity of } \mu \text { in } V=\operatorname{def}_{\operatorname{mult}_{V}}(\mu) .
$$

The $(\mathfrak{h}, L(\mathbb{C}))$-module $V$ is called admissible if $\operatorname{mult}_{V}(\mu)<\infty$ for all $\mu$.

In terms of the multiplicity spaces, we can calculate Hermitian dual spaces:

$$
V(\mu)^{h} \simeq V^{\mu, h} \otimes E_{\mu}^{h} .
$$

We fix now a real structure $\sigma$ on the pair (Definition 8.1). The real structure $\sigma$ on $L(\mathbb{C})$ defines via $(8.5 \mathrm{~h})$ an algebraic representation $\sigma(\mu)$ on $E_{\mu}^{h}$. The assumed surjectivity of $\sigma$ on $L(\mathbb{C})$ implies that $\sigma(\mu)$ is irreducible. The map $\mu \mapsto \sigma(\mu)$ of irreducible representations of $L(\mathbb{C})$ is bijective, and $\sigma^{2}(\mu)$ is naturally isomorphic to $\mu$. We therefore find

$$
V^{h}=\sum_{\mu \in \widehat{L(\mathbb{C})}} V^{\mu, h} \otimes E_{\sigma(\mu)} .
$$

(This uses the redefinition of Hermitian dual in (8.2f). Without that, we would instead have the direct product over $\mu$ as the Hermitian dual of the direct sum.)

We will often (but not always) be interested in the special case that

$$
\sigma \text { is a compact real form of } L(\mathbb{C})
$$

with (compact) group of real points $L$. In this special case we fix also an $L$-invariant unitary structure - a positive definite $L$-invariant Hermitian form —on each space $E_{\mu}$. This amounts to a choice of isomorphism

$$
E_{\sigma(\mu)} \simeq E_{\mu} .
$$

In particular, we must have $\sigma(\mu) \simeq \mu$ whenever (8.8g) holds. Since $E_{\mu}$ and $E_{\sigma(\mu)}$ are irreducible, the space of such isomorphisms has complex dimension one. Requiring the form to be Hermitian defines a real line in that complex line, and the positivity defines a real half line. 
Proposition 8.9. Suppose $(\mathfrak{h}, L(\mathbb{C}))$ is a pair with $L(\mathbb{C})$ reductive, and $\sigma$ \{prop:sigchar\} is a real structure. (Definition 8.1).

1. Suppose $V$ is an admissible $(\mathfrak{h}, L(\mathbb{C})$ )-module (see (8.8)). Then the $\sigma$-Hermitian dual module $V^{h, \sigma}$ is again admissible, with multiplicity spaces

$$
\left(V^{h, \sigma}\right)^{\sigma(\mu)} \simeq\left(V^{\mu}\right)^{h}
$$

2. The space of $\sigma$-invariant sesquilinear forms $\langle,\rangle_{T}$ on $V$ may be identified (by (8.5j)) with the complex vector space of intertwining operators $\operatorname{Hom}_{\mathfrak{h}, L(\mathbb{C})}\left(V, V^{h, \sigma}\right)$. This complex vector space has a real structure $\bar{T}={ }_{\text {def }} T^{h}$; the subspace of real points consists of the Hermitian $\sigma$ invariant forms.

3. Suppose $J$ is an admissible irreducible $(\mathfrak{h}, L(\mathbb{C}))$-module (see (8.8)). Then the $\sigma$-Hermitian dual module $J^{h, \sigma}$ is again admissible and irreducible. Consequently $J$ admits a nonzero invariant sesquilinear form if and only if $J \simeq J^{h, \sigma}$. In this case the form is necessarily nondegenerate and unique up to a nonzero complex scalar multiple.

Assume from now on, as in $(8.8 \mathrm{~g})$, that $\sigma$ defines a compact real form of $L$.

4. Fix a $\sigma$-invariant Hermitian form $\langle,\rangle_{V}$ on $V$. The decomposition

$$
V=\sum_{\mu \in \widehat{L}} V^{\mu} \otimes E_{\mu}
$$

(cf. (8.8)) is orthogonal. The chosen unitary structure $\langle,\rangle_{\mu}$ on each irreducible representation $\mu$ of $L$ gives rise to a natural Hermitian form $\langle,\rangle_{V}^{\mu}$ on each multiplicity space $V^{\mu}$ so that

$$
\left(V,\langle,\rangle_{V}\right)=\sum_{\mu \in \widehat{L}}\left(V^{\mu},\langle,\rangle_{V}^{\mu}\right) \otimes\left(E_{\mu},\langle,\rangle_{\mu}\right) .
$$

5. Define

$$
\begin{aligned}
& \operatorname{pos}_{V}(\mu)=\text { dimension of maximal positive definite subspace of } V^{\mu} \\
& \operatorname{neg}_{V}(\mu)=\text { dimension of maximal negative definite subspace of } V^{\mu} \\
& \operatorname{rad}_{V}(\mu)=\text { dimension of radical of } V^{\mu} .
\end{aligned}
$$

Then

$\left(\operatorname{pos}_{V}, \operatorname{neg}_{V}, \operatorname{rad}_{V}\right): \widehat{L} \rightarrow \mathbb{N} \times \mathbb{N} \times \mathbb{N}, \quad \operatorname{pos}_{V}+\operatorname{neg}_{V}+\operatorname{rad}_{V}=\operatorname{mult}_{V}$.

Here mult $_{V}(\mu)$ is the multiplicity of $\mu$ in $V$ (cf. (8.8)). 
6. Assume again that $J$ is irreducible and that $J \simeq J^{h, \sigma}$, so that there is a nondegenerate invariant Hermitian form $\langle,\rangle_{J}$. Multiplying this form by a negative scalar interchanges $\operatorname{pos}_{J}$ and $\operatorname{neg}_{J}$. The unordered pair of functions $\left\{\operatorname{pos}_{J}, \operatorname{neg}_{J}\right\}$ is independent of the choice of form.

All of this is straightforward and elementary.

We call the triple of functions $\left(\operatorname{pos}_{V}, \operatorname{neg}_{V}, \operatorname{rad}_{V}\right)$ the signature character of $\left(V,\langle,\rangle_{V}\right)$. The form is nondegenerate if and only if $\operatorname{rad}_{V}=0$; in that case we will often omit it from the notation.

Corollary 8.10. In the setting of Proposition 8.9, the admissible irreducible $(\mathfrak{h}, L(\mathbb{C})$ )-module $J$ admits a positive definite $\sigma$-invariant Hermitian form if and only if

1. $J$ is equivalent to the Hermitian dual irreducible $J^{h, \sigma}$, and

2. one of the functions $\operatorname{pos}_{J}$ or neg ${ }_{J}$ is identically zero.

This corollary begins to suggest why one can hope to calculate the unitary dual: the problem is not whether some matrix is positive definite (which sounds analytic, difficult, and subject to subtle error analysis) but whether some nonnegative integers are zero (which sounds combinatorial). In section 15 we will describe techniques from [43] for reducing further to a computation of finitely many integers.

We will need to consider Hermitian forms invariant under several different real structures on the same pair. Here is some machinery for doing that.

Definition 8.11. Suppose $(\mathfrak{h}, L(\mathbb{C}))$ is a pair (Definition 8.1). An automorphism $\delta$ of the pair consists of a complex Lie algebra automorphism

$$
\delta: \mathfrak{h} \rightarrow \mathfrak{h}
$$

and an algebraic group automorphism

$$
\delta: L(\mathbb{C}) \rightarrow L(\mathbb{C}),
$$

subject to the requirement that the differential of the group automorphism is equal to the restriction to $\mathfrak{l}$ of the Lie algebra automorphism.

If $V$ is a representation of the pair (Definition 8.1), then the twist of $V$ by $\delta$ is the representation $V^{\delta}$ on the same vector space $V$ defined by

$$
X \cdot_{\delta} v=\delta(X) \cdot v, \quad \ell \cdot{ }_{\delta} v=\delta(\ell) \cdot v \quad(X \in \mathfrak{h}, \ell \in L(\mathbb{C})) .
$$


Twisting defines a right action of the group of automorphisms on representations:

$$
\left(V^{\delta_{1}}\right)^{\delta_{2}}=V^{\delta_{1} \circ \delta_{2}}
$$

A $\delta$-form of $V$ is an isomorphism

$$
D: V \rightarrow V^{\delta} .
$$

That is, $D$ is a linear automorphism of the vector space $V$ satisfying

$D(X \cdot v)=\delta(X) \cdot D(v), \quad D(\ell \cdot v)=\delta(\ell) \cdot D(v) \quad(X \in \mathfrak{h}, \ell \in L(\mathbb{C}))$.

This last definition in particular may look more transparent if we use representation notation: writing $\pi$ for the representations of $\mathfrak{h}$ and $L(\mathbb{C})$ on $V$, and $\pi^{\delta}$ for their twist by $\delta$, the condition is

$$
D \pi=\pi^{\delta} D
$$

that is, that $D$ intertwines the representations $\pi$ and $\pi^{\delta}$.

Definition 8.12. Suppose $(\mathfrak{h}, L(\mathbb{C}))$ is a pair, and $\delta$ is an automorphism $\{$ def : extpair $\}$ with the property that

$$
\delta^{m}=\operatorname{Ad}(\lambda) \quad(\lambda \in L(\mathbb{C}))
$$

is an inner automorphism (Definition 8.11). The corresponding extended group is the extension

$$
1 \rightarrow L(\mathbb{C}) \rightarrow{ }^{\delta} L(\mathbb{C}) \rightarrow \mathbb{Z} / m \mathbb{Z} \rightarrow 1
$$

with a specified generator $\delta_{1}$ mapping to $1 \in \mathbb{Z} / m \mathbb{Z}$, and subject to the relations

$$
\delta_{1} \ell \delta_{1}^{-1}=\delta(\ell) \quad(\ell \in L(\mathbb{C})),
$$

and

$$
\delta_{1}^{m}=\lambda .
$$

This extended group acts by automorphisms on $\mathfrak{h}$, and we call $\left(\mathfrak{h},{ }^{\delta} L(\mathbb{C})\right)$ an extended pair.

It is convenient to abuse notation by writing $\delta$ for the distinguished generator $\delta_{1}$

$$
{ }^{\delta} L(\mathbb{C})=L(\mathbb{C})\left\{e, \delta, \delta^{2}, \ldots, \delta^{m-1}\right\},
$$

a union of $m$ cosets of $L(\mathbb{C})$.

We write $\chi$ for the one-dimensional $\left(\mathfrak{h},{ }^{\delta} L(\mathbb{C})\right)$-module $\mathbb{C}_{\chi}$ defined by

$X \cdot z=0, \quad \ell \cdot z=z, \quad \delta \cdot z=e^{2 \pi i / m} z \quad\left(X \in \mathfrak{h}, \ell \in L(\mathbb{C}), z \in \mathbb{C}_{\chi}\right)$. 
Proposition 8.13. Suppose we are in the setting of Definition 8.12.

1. Twisting by $\delta$ defines a permutation of order (dividing) $m$ of the set of irreducible $(\mathfrak{h}, L(\mathbb{C}))$-modules. Tensoring with $\chi$ defines a permutation of order (dividing) $m$ of the set of irreducible $\left(\mathfrak{h},{ }^{\delta} L(\mathbb{C})\right)$-modules.

Now fix an irreducible $(\mathfrak{h}, L(\mathbb{C}))$-module $V$, and write $\Lambda \in \operatorname{Aut}(V)$ for the action of $\lambda=\delta^{m}$.

2. The module $V$ is fixed by $\delta$ if and only if there is a $\delta$-form $D$ of $V$ (Definition 8.11); that is, an intertwining operator from $V$ to $V^{\delta}$.

3. If $D$ exists for $V$, then it is unique up to multiplication by a complex scalar; and $D^{m}$ must be a scalar multiple of $\Lambda$.

4. If $D$ exists for $V$, then we can arrange $D^{m}=\Lambda$. With this constraint, $D$ is unique up to multiplication by an mth root of 1 .

5. The irreducible $(\mathfrak{h}, L(\mathbb{C}))$-module $V$ extends to $\left(\mathfrak{h},{ }^{\delta} L(\mathbb{C})\right)$ if and only if $V \simeq V^{\delta}$. In this case there are exactly $m$ extensions, which are cyclically permuted by tensoring with the character $\chi$ of Definition 8.12. The actions of $\delta$ are given by the $m \delta$-forms $D$ described in (4).

6. The irreducible $(\mathfrak{h}, L(\mathbb{C}))$-module $V$ induces irreducibly to $\left(\mathfrak{h},{ }^{\delta} L(\mathbb{C})\right)$ if and only if the $m$ twists $V, V^{\delta}, \ldots, V^{\delta^{m-1}}$ are all inequivalent. In this case

$$
\operatorname{Ind}_{(\mathfrak{h}, L(\mathbb{C}))}^{(\mathfrak{h}, \delta(\mathbb{C}))}(V)=V \oplus V^{\delta} \oplus \cdots \oplus V^{\delta^{m-1}}
$$

is the unique irreducible $\left(\mathfrak{h},{ }^{\delta} L(\mathbb{C})\right)$-module containing $V$. This induced module is isomorphic to its tensor product with the character $\chi$ of Definition 8.12.

This analysis of representations of cyclic group extensions is often referred to as "Clifford theory" ([6], pages 547-548), although much of it goes back at least to Frobenius.

Definition 8.14. In the setting of Definition 8.12, the modules arising $\{$ def : typeext $\}$ in (5) are called type 1 , and those arising in (6) type $m$, for the multiplicity on restriction to $(\mathfrak{h}, L(\mathbb{C}))$. In the same way, we call an irreducible $(\mathfrak{h}, L(\mathbb{C}))$-module type one if its equivalence class is fixed by $\delta$, and type $m$ if the orbit under twisting has order $m$.

There is no difficulty in analyzing the intermediate cases between (5) and (6), when the equivalence class of $V$ is fixed by some power $\delta^{m_{0}}$ 
(with $m_{0}$ a proper divisor of $m$ ). Since we will be interested in the case $m=2$, we have omitted these type $m_{0}$ cases.

We turn now to an analysis of sesquilinear and Hermitian forms for extended groups.

Lemma 8.15. Suppose $(\mathfrak{h}, L(\mathbb{C}))$ is a pair endowed with a real structure $\sigma$ \{lem: changeform\} (Definition 8.1); we do not require that $\sigma^{2}=1$. Suppose also that

a) $\epsilon$ and $\delta$ are two automorphisms of the pair (Definition 8.11);

b) $V, W, V^{\prime}$, and $W^{\prime}$ are four $(\mathfrak{h}, L(\mathbb{C}))$-modules;

c) $\langle\cdot, \cdot\rangle: V \times W \rightarrow \mathbb{C}$ is a $\sigma$-invariant sesquilinear pairing ((8.2)); and

d) $D: V^{\prime} \rightarrow V^{\delta}$ and $E: W^{\prime} \rightarrow W^{\epsilon}$ are $(\mathfrak{h}, L(\mathbb{C}))$-maps to the indicated twisted modules (Definition 8.11).

Then the form

$$
\langle\cdot, \cdot\rangle^{\prime}: V^{\prime} \times W^{\prime} \rightarrow \mathbb{C}, \quad\left\langle v^{\prime}, w^{\prime}\right\rangle^{\prime}={ }_{\operatorname{def}}\left\langle D v^{\prime}, E w^{\prime}\right\rangle \quad\left(v^{\prime} \in V^{\prime}, w^{\prime} \in W^{\prime}\right)
$$

is an $\epsilon^{-1} \sigma \delta$-invariant sesquilinear pairing.

This is immediate from the definitions.

Proposition 8.16. Suppose $(\mathfrak{h}, L(\mathbb{C}))$ is a pair endowed with two real struc\{prop: changeform\} tures $\sigma_{1}$ and $\sigma_{2}$ (Definition 8.1); we assume that $\sigma_{i}^{2}=1$. Suppose $V$ is an admissible $(\mathfrak{h}, L(\mathbb{C}))$-module, and that $V$ is endowed with a nondegenerate $\sigma_{1}$-invariant Hermitian form $\langle,\rangle^{\sigma_{1}}$ that is unique up to real multiple. Then

1. The maps

$$
\delta=\sigma_{1}^{-1} \sigma_{2}, \quad \epsilon=\sigma_{2} \sigma_{1}^{-1}
$$

define automorphisms of the pair (Definition 8.11).

2. Since $\sigma_{i}^{2}=1, \delta$ and $\epsilon$ are inverse to each other. It is also the case that they are conjugate by $\sigma_{1}: \sigma_{1} \delta \sigma_{1}^{-1}=\epsilon$.

3. The $\sigma_{1}$ - and $\sigma_{2}$-hermitian duals of $V$ differ by twisting by $\delta$ or $\epsilon$ :

$$
V^{h, \sigma_{2}} \simeq\left[V^{h, \sigma_{1}}\right]^{\delta}, \quad\left[V^{\epsilon}\right]^{h, \sigma_{1}} \simeq V^{h, \sigma_{2}} .
$$

(notation (8.5h) and Definition 8.11).

4. The following conditions are equivalent. 
(a) There is a nondegenerate $\sigma_{2}$-invariant form $\langle,\rangle^{\sigma_{2}}$ on $V$. In this case the form is unique up to a real multiple.

(b) $V$ is equivalent to its twist by $\delta$, by an isomorphism

$$
D: V \rightarrow V^{\delta}
$$

(cf. Definition 8.11). In this case the isomorphism $D$ is unique up to a complex multiple.

(c) $V$ is equivalent to its twist by $\epsilon$, by an isomorphism

$$
E: V \rightarrow V^{\epsilon}
$$

In this case the isomorphism $E$ is unique up to a complex multiple. One candidate for $E$ is $D^{-1}$.

Assume now that $\delta^{m}=\operatorname{Ad}(\lambda)(\lambda \in L(\mathbb{C}))$ as in Definition 8.12; equivalently, that $\epsilon^{m}=\operatorname{Ad}\left(\lambda^{-1}\right)=\operatorname{Ad}\left(\sigma_{1}(\lambda)\right)$. Write $\Lambda$ for the action on $V$ of $\lambda$, and $\Lambda_{1}$ for the action of $\sigma_{1}(\lambda)$. Then these conditions are also equivalent to the following ones.

(d) The $(\mathfrak{h}, L(\mathbb{C}))$-module $V$ extends to an $\left(\mathfrak{h},{ }^{\delta} L(\mathbb{C})\right)$-module, with $\delta$ acting by an isomorphism

$$
D: V \rightarrow V^{\delta}
$$

as in (b), subject to the additional requirement $D^{m}=\Lambda$. If any such extension exists then there are exactly $m$, the operators $D$ differing by multiplication by an mth root of 1 .

(e) The $(\mathfrak{h}, L(\mathbb{C}))$-module $V$ extends to an $\left(\mathfrak{h},{ }^{\epsilon} L(\mathbb{C})\right)$-module, with $\epsilon$ acting by an isomorphism

$$
E: V \rightarrow V^{\epsilon}
$$

as in (b), subject to the additional requirement $E^{m}=\Lambda_{1}$. If any such extension exists then there are exactly $m$, the operators $E$ differing by multiplication by an mth root of 1 . One candidate for $E$ is the inverse Hermitian transpose of $D$ (defined using $\langle,\rangle^{\sigma_{1}}$ ).

5. If the automorphism $\delta=\operatorname{Ad}(d)$ is inner (that is, given by the adjoint action of an element $d \in L(\mathbb{C})$ with $d^{m}=\lambda$ ) then the conditions of (4) are automatically satisfied; the intertwining operator $D$ may be taken as the action of $d$, and $E$ as the action of $\sigma_{1}(d)$. 
Assume now that the conditions of (4) are satisfied, that $\delta^{m}=\operatorname{Ad}(\lambda)$, and that the operators $D$ and $E$ are chosen as in $(4)(d)$ and $(4)(e)$.

6. There is a nonzero complex number $\xi(D, E)$ so that

$$
E^{-1}=\xi(D, E) D .
$$

The scalar $\xi(D, E)$ is an mth root of 1 .

7. The form

$$
\langle v, w\rangle^{\prime}={ }_{\operatorname{def}}\langle D v, E w\rangle^{\sigma_{1}}
$$

is a $\sigma_{1}$-invariant sesquilinear form on $V((8.5 \mathrm{j}))$, and by hypothesis in (4) is therefore a multiple of $\langle,\rangle^{\sigma_{1}}$ :

$$
\langle D v, E w\rangle^{\sigma_{1}}=\omega(D, E)\langle v, w\rangle^{\sigma_{1}} .
$$

Equivalently,

$$
\langle D v, w\rangle^{\sigma_{1}}=\omega(D, E)\left\langle v, E^{-1} w\right\rangle^{\sigma_{1}}=\omega(D, E) \overline{\xi(D, E)}\langle v, D w\rangle^{\sigma_{1}} .
$$

8. The scalar $\omega(D, E)$ satisfies

$$
\omega(D, E)^{m}=1, \quad \omega\left(\tau_{1} D, \tau_{2} E\right)=\tau_{1} \tau_{2}^{-1} \omega(D, E) \quad\left(\tau_{i} \in \mathbb{C}^{\times}, \tau_{i}^{m}=1\right) .
$$

9. If $\zeta$ is any square root of $\omega(D, E) \overline{\xi(D, E)}$, then

$$
\langle v, w\rangle^{\sigma_{2}}={ }_{\operatorname{def}} \zeta^{-1}\langle D v, w\rangle^{\sigma_{1}}=\zeta\langle v, D w\rangle^{\sigma_{1}}
$$

is a $\sigma_{2}$-invariant Hermitian form on $V$.

Proof. Parts (1), (2), and (3) are immediate from the definitions. The equivalence of (a)-(c) in (4) is also easy, and then (d)-(e) is a consequence of Proposition 8.13.

The existence of the scalar in part (6) follows from the description of $E$ in (4)(c). Raising this equation to the $m$ th power gives

$$
\Lambda_{1}^{-1}=\xi(D, E)^{m} \Lambda
$$

The operators $\Lambda$ and $\Lambda_{1}^{-1}$ are Hermitian transposes of each other with respect to the form $\langle,\rangle^{\sigma_{1}}$. Taking Hermitian transpose of the last equation gives

$$
\Lambda=\overline{\xi(D, E)}^{m} \Lambda_{1}^{-1}
$$


Combining these two equations gives $|\xi(D, E)|^{2 m}=1$, which is the last claim in (6).

Part (7) follows from Lemma 8.15.

For (8), applying the formula in (7) $m$ times gives

$$
\left\langle D^{m} v, w\right\rangle^{\sigma_{1}}=\omega(D, E)^{m}\left\langle v, E^{-m} w\right\rangle^{\sigma_{1}} .
$$

Again using the fact that the operators $D^{m}=\Lambda$ and $E^{-m}=\Lambda_{1}^{-1}$ are Hermitian transposes of each other, it follows that $\omega(D, E)^{m}=1$. The formula with $\tau_{i}$ is easy.

For (9), that $\langle v, w\rangle^{\sigma_{2}}=\langle D v, w\rangle^{\sigma_{1}}$ is $\sigma_{2}$-invariant and sesquilinear follows from Lemma 8.15. That the indicated multiple is Hermitian is an easy consequence of the last formula

$$
\zeta^{-1}\langle D v, w\rangle^{\sigma_{1}}=\zeta\langle v, D w\rangle^{\sigma_{1}}=\overline{\zeta^{-1}\langle D w, v\rangle^{\sigma_{1}}}
$$

in (7). (We need to use that $\bar{\zeta}=\zeta^{-1}$, which follows from (6) and (8).)

\section{Interlude: realizing standard modules}

We sketched briefly in (6.4) Langlands' original realization of the standard $\{$ sec:stdmods $\}$ $\left(\mathfrak{g}_{0}, K\right)$-modules using parabolic induction from discrete series representations. From time to time we are going to need other realizations of the standard modules, notably those found by Zuckerman and described in [38]. The purpose of this section is to collect some general facts about these other realizations, which require some time to state precisely. The reader will probably prefer to pass over this section, and consult it only as necessary later on.

The main theorems of this section have their origins in [38], Theorem 6.6.15 (which is not proven there) and [41], Theorem 1.13 (which considers only integral infinitesimal character). Results in the generality that we need, with proofs, may be found in [21], Chapter 11; but translating the formulation there into what we want is still a bit of an exercise, most of which is silently left to the reader.

We begin as in (6.4) with a $\theta$-stable Cartan subgroup

$$
H=T A \subset G, \quad T=H^{\theta}=H \cap K, \quad A=\exp \left(\mathfrak{h}_{0}^{-\theta}\right) .
$$

and a weak Langlands parameter (Definition 6.3)

$$
\Gamma=\left(H, \gamma, R_{i \mathbb{R}}^{+}\right)=(\Lambda, \nu)
$$


Just as in (6.4), we consider the real Levi subgroup

$$
M A=\text { centralizer in } G \text { of } A \supset H .
$$

The roots of $H$ in $M A$ are precisely the imaginary roots (Definition 5.7); so what is left are the non-imaginary roots. A real parabolic subgroup $P=M A N$ of $G$ is called type $L$ with respect to $\Gamma$ (see [21], page 704) if whenever $\alpha$ is a (necessarily non-imaginary) root of $\mathfrak{h}$ in $\mathfrak{g}$ such that

$$
\left\langle d \gamma, \alpha^{\vee}\right\rangle \in \mathbb{Z}^{>0} \quad \text { and } \quad\left\langle d \gamma,-\theta \alpha^{\vee}\right\rangle \geq 0
$$

$\{$ eq: typeL $\}$

then $\alpha$ is a root of $H$ in $\mathfrak{n}$. The "L" stands for "Langlands," since we will use this hypothesis to make Langlands' construction of standard modules. Notice that "type L" is a weaker hypothesis than "Re $\nu$ weakly dominant," which we assumed in (6.4). Notice also that the parabolic subgroup $P=$ $M A N$ is pointwise fixed by the real form $\sigma_{0}$, and that the Cartan involution $\theta$ carries $P$ to the opposite parabolic subgroup

$$
P^{\mathrm{op}}=M A N^{\mathrm{op}}=M A \theta(N)=\theta(P) .
$$

Theorem 9.2 ([21], Theorem 11.129). Suppose $\Gamma=(\Lambda, \nu)$ is a weak Lang$\{$ eq:Pop $\}$ $\{$ thm:Lrealiz\} lands parameter, and $P=M A N$ is a parabolic subgroup of type $L$ with respect to $\Gamma((9.1))$. Then the standard quotient-type module

$$
I_{\text {quo }}(\Gamma)=\operatorname{Ind}_{M A N}^{G}(D(\Lambda) \otimes \nu \otimes 1)
$$

is independent of the choice of $P$ (of type $L$ ). (Here we write $D(\Lambda)$ for the limit of discrete series representation of $M$ introduced in (6.4).) It is a direct sum of distinct Langlands standard quotient-type modules (Theorem 6.1), so its largest completely reducible quotient $J(\Gamma)$ is the corresponding direct sum of distinct irreducible modules.

In the same way, the standard sub-type module

$$
\left.I_{\text {sub }}(\Gamma)=\operatorname{Ind}_{M A N^{o p}}^{G} D(\Lambda) \otimes \nu \otimes 1\right)
$$

is independent of the choice of $P$ (of type L). It is a direct sum of distinct Langlands standard sub-type modules (Theorem 6.1), so its socle $J(\Gamma)$ is the corresponding direct sum of distinct irreducible modules.

The two keys to making this construction are Mackey's notion of induction and Harish-Chandra's description of the limit of discrete series representation $D(\Lambda)$ for $M$. The representation $D(\Lambda)$ can be constructed using Zuckerman's "cohomological induction" functor for the Borel subalgebra of 
$\mathfrak{m}$ determined by $R_{i \mathbb{R}}^{+}$. (This is a consequence of Theorem 9.4 specialized to the case when $H$ is compact; the result, due to Zuckerman, was one of his original motivations for introducing cohomological induction.)

In an exactly parallel way, still referring to a weak Langlands parameter $\{$ se:stdVZparam $\}$ $\Gamma$ as above, we can consider the subgroup

$$
L=\text { centralizer in } G \text { of } T_{0} \supset H .
$$

The roots of $H$ in $L$ are precisely the real roots (Definition 5.7); so what is left are the non-real roots. A $\theta$-stable parabolic subalgebra $\mathfrak{q}=\mathfrak{l}+\mathfrak{u}$ of $\mathfrak{g}$ is called type $V Z$ with respect to $\Gamma$ (see [21], page 706) if (first) every root in $R_{i \mathbb{R}}^{+}$is a root in $\mathfrak{u}$; and (second) whenever $\alpha$ is a (necessarily non-real) root of $\mathfrak{h}$ in $\mathfrak{g}$ such that

$$
\left\langle d \gamma, \alpha^{\vee}\right\rangle \in \mathbb{Z}^{>0} \quad \text { and } \quad\left\langle d \gamma, \theta \alpha^{\vee}\right\rangle \geq 0,
$$

$\{$ eq: typeVZ $\}$

then $\alpha$ is a root of $H$ in $\mathfrak{u}$. The "VZ" stands for "Zuckerman," since we will use this hypothesis to make Zuckerman's construction of standard modules.

Notice that $\mathfrak{l}$ is preserved by both the real form $\sigma_{0}$ and by $\theta$. The parabolic $\mathfrak{q}$ is preserved by $\theta$, but the real form $\sigma_{0}$ sends $\mathfrak{q}$ to the opposite parabolic

$$
\mathfrak{q}^{\text {op }}=\mathfrak{l}+\mathfrak{u}^{\text {op }}=\mathfrak{l}+\overline{\mathfrak{u}}=\overline{\mathfrak{q}} .
$$

These properties are complementary to those of the real parabolic $P$ described in (9.1e).

To describe Zuckerman's construction, we begin by constructing a weak Langlands parameter

$$
\Gamma_{\mathfrak{q}}=\left(H, \gamma_{\mathfrak{q}}, \emptyset\right)=\left(\Lambda_{\mathfrak{q}}, \nu\right) \in \widehat{T} \times \mathfrak{a}^{*}
$$

$\{$ eq: gammaq1\}

for $L$ with differential

$$
d \gamma_{\mathfrak{q}}=d \gamma-\rho(\mathfrak{u}) \in \mathfrak{h}^{*}
$$

here $\rho(\mathfrak{u})$ is half the sum of the roots of $H$ in $\mathfrak{u}$. We can write

$$
\Delta(\mathfrak{u}, H)=R_{i \mathbb{R}}^{+} \cup \bigcup_{\text {complex pairs in } \mathfrak{u}}\{\alpha, \theta \alpha\} .
$$

Recall that $\gamma$ is a character of the $\rho_{i \mathbb{R}}$ cover of $H$, and this is the same as a character $\gamma \otimes \rho_{i \mathbb{R}}^{-1}$ of $H$. We define

$$
\left.\left.\gamma_{\mathfrak{q}}\right|_{T}=\gamma \otimes \rho_{i \mathbb{R}}^{-1}-\left.\sum_{\text {complex pairs } \alpha, \theta \alpha \text { in } \mathfrak{u}} \alpha\right|_{T},\left.\quad \gamma_{\mathfrak{q}}\right|_{A}=\left.\gamma\right|_{A}=\nu ; \quad \text { (9.3g) } \quad \text { eq:gammaq }\right\}
$$


since the two roots $\alpha$ and $\theta \alpha$ have the same restriction to $T$, the formula in $(9.3 \mathrm{~g})$ is well defined (independent of the choice of one root in each complex pair) and has differential $d \gamma-\rho(\mathfrak{u})$.

Now we can form the standard representation $I_{\text {quo }}\left(\Gamma_{\mathfrak{q}}\right)$ of $L$. Because it is a fundamental building block in Zuckerman's construction, we recall how this representation of $L$ is defined. By construction of $L$, the roots of $H$ in $L$ are all real; so by choosing a system of positive real roots, we can construct a Borel subgroup

$$
B_{L}=T A N_{L}
$$

of $L$. According to $(9.1 \mathrm{~d})$, we can and do require that $B_{L}$ be of "type L." This means that $d \gamma_{\mathfrak{q}}$ should be "integrally dominant": for every real root $\alpha$ of $H$ in $\mathfrak{g}$, we require that if

$$
\left\langle d \gamma_{\mathfrak{q}}, \alpha^{\vee}\right\rangle=\left\langle\nu, \alpha^{\vee}\right\rangle \in \mathbb{Z}^{>0}
$$

then $\alpha$ is a root of $H$ in $\mathfrak{n}_{L}$. If we have already a parabolic subgroup $P$ of type $\mathrm{L}$ with respect to $\Gamma$, then $B_{L}=P \cap L$ is of type $\mathrm{L}$ with respect to $\Gamma_{\mathfrak{q}}$.

With such a choice of $B_{L}$, we have

$$
I_{\text {quo }}\left(\Gamma_{\mathfrak{q}}\right)=\operatorname{Ind}_{T A N_{L}}^{L}\left(\left.\gamma_{\mathfrak{q}}\right|_{T} \otimes \nu \otimes 1\right),
$$

an ordinary principal series representation of $L$. Similarly,

$$
I_{\text {sub }}\left(\Gamma_{\mathfrak{q}}\right)=\operatorname{Ind}_{T A N_{L}^{\mathrm{op}}}^{L}\left(\left.\gamma_{\mathfrak{q}}\right|_{T} \otimes \nu \otimes 1\right),
$$

Because of the realization of the standard module $I_{\text {quo }}(\Gamma)$ in Theorem 9.4, certain simple properties of this standard module correspond to simple properties of the minimal principal series representation $I_{\text {quo }}\left(\Gamma_{\mathfrak{q}}\right)$ for the split group $L$. By induction by stages, these properties in turn come down to properties of principal series representations for the real root $S L(2, \mathbb{R})$ subgroups. For this reason, many statements (especially around the KazhdanLusztig conjectures) will involve the relationship of real roots to the parameter $\Gamma_{\mathfrak{q}}$ of $(9.3 \mathrm{~d})$ above.

The hypothesis $(9.3 \mathrm{~b})$ says that $I_{\text {quo }}\left(\Gamma_{\mathfrak{q}}\right)$ is somewhat "dominant" with respect to the parabolic $\mathfrak{q}$, and therefore somewhat "negative" with respect to $\overline{\mathfrak{q}}$. The two highest weight modules

$$
M_{\mathfrak{q}, \text { quo }}(\Gamma)=U(\mathfrak{g}) \otimes_{\overline{\mathfrak{q}}}\left[I_{\text {quo }}\left(\Gamma_{\mathfrak{q}}\right) \otimes \wedge^{\text {top }}(\mathfrak{u})\right]
$$

and

$$
M_{\mathfrak{q}, \operatorname{sub}}(\Gamma)=\operatorname{Hom}_{\mathfrak{q}}\left(U(\mathfrak{g}),\left[I_{\text {sub }}\left(\Gamma_{\mathfrak{q}}\right) \otimes \wedge^{\text {top }}(\mathfrak{u})\right]\right)_{L \cap K \text {-finite }}
$$


are $(\mathfrak{g}, L \cap K)$-modules of infinitesimal character $d \gamma$. ("Somewhat dominant" means that these modules are trying to be irreducible, like a Verma module with an antidominant highest weight. If the hypothesis $(9.3 \mathrm{~b})$ were strengthened to

$$
\left\langle d \gamma, \alpha^{\vee}\right\rangle \notin \mathbb{Z}^{<0} \quad(\alpha \in \Delta(\mathfrak{u}, \mathfrak{h}))
$$

then $M_{\mathfrak{q}, q u o}(\Gamma)$ and $M_{\mathfrak{q} \text {,sub }}(\Gamma)$ would be (isomorphic) irreducible modules. The advantage of $(9.3 \mathrm{~b})$ is that for any $\Gamma$ we can choose $\mathfrak{q}$ of type VZ; but there are many $\Gamma$ for which no choice of $\mathfrak{q}$ satisfies $(9.3 \mathrm{n})$.)

The two modules $M_{\mathfrak{q}, \text { quo }}(\Gamma)$ and $M_{\mathfrak{q}, \text { sub }}(\Gamma)$ have exactly the same restriction to $L \cap K$, and in fact (as is elementary to see) exactly the same irreducible composition factors.

Here is the realization of the standard modules using Zuckerman's functors.

Theorem 9.4 ([21], Theorem 11.129). Suppose $\Gamma=(\Lambda, \nu)$ is a weak Lang$\{$ thm:VZrealiz\} lands parameter, and $\mathfrak{q}=\mathfrak{l}+\mathfrak{u}$ is a $\theta$-stable parabolic subalgebra of type $V Z$ with respect to $\Gamma((9.3))$. Define $s=\operatorname{dim}(\mathfrak{u} \cap \mathfrak{k})$. Then the standard quotient-type module of Theorem 9.2 may also be realized by cohomological induction from $\overline{\mathfrak{q}}$ :

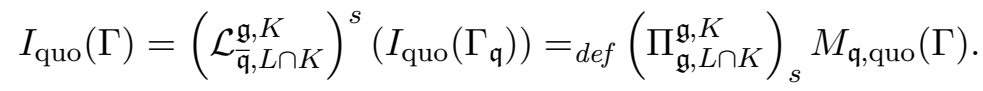

The derived functors vanish in all other degrees. This module is therefore a direct sum of distinct Langlands standard quotient-type modules (Theorem 6.1), so its largest completely reducible quotient $J(\Gamma)$ is the corresponding direct sum of distinct irreducible modules.

In the same way, the standard sub-type module may be realized as

$$
I_{\text {sub }}(\Gamma)=\left(\mathcal{R}_{\mathfrak{q}, L \cap K}^{\mathfrak{g}, K}\right)^{s}\left(I_{\text {sub }}\left(\Gamma_{\mathfrak{q}}\right)\right)=\operatorname{def}\left(\Gamma_{\mathfrak{g}, L \cap K}^{\mathfrak{g}, K}\right)^{s} M_{\mathfrak{q}, \text { sub }}(\Gamma)
$$

The derived functors vanish in all other degrees. This is therefore a direct sum of distinct Langlands standard sub-type modules (Theorem 6.1), so its socle $J(\Gamma)$ is the corresponding direct sum of distinct irreducible modules.

The functor $\Gamma_{\mathfrak{g}, L \cap K}^{\mathfrak{g}, K}$ appearing here is Zuckerman's "largest $K$-finite submodule" functor ([38], Definition 6.2.9, or [21], pages 101-107), and the superscripts are its right derived functors. The functor $\Pi_{\mathfrak{g}, L \cap K}^{\mathfrak{g}, K}$ is "largest $K$-finite quotient" ([21], pages 101-107), sometimes called a "Bernstein functor" because of lectures given by Bernstein in 1983 featuring a version of this functor. The subscripts are left derived functors. 
The two keys to making this construction are Zuckerman's cohomological parabolic induction functors, and (Mackey's) construction of the ordinary principal series representations $I\left(\Gamma_{\mathfrak{q}}\right)$ for $L$.

In order to describe Kazhdan-Lusztig polynomials in Sections 18 and 19, it will be helpful to recall one of the technical tools used to prove Theorem 9.4. (Indeed this is the tool used in [38] to prove Theorem 6.1.) That is Lie algebra cohomology.

Proposition 9.5. Suppose $\Gamma=\left(T A, \gamma, R_{i \mathbb{R}}^{+}\right)$is a weak Langlands parameter, \{prop:ubarcohom\} $L$ is the centralizer in $G$ of $T_{0}$, and

$$
\mathfrak{q}=\mathfrak{l}+\mathfrak{u}
$$

is a $\theta$-stable parabolic subalgebra of type $V Z$ with respect to $\Gamma$ (see (9.3b)). Define $\Gamma_{\mathfrak{q}}$, a weak Langlands parameter for $L$ (that is, a character of $H$ ) as in $(9.3 \mathrm{~g})$. Suppose $X$ is a $(\mathfrak{g}, K)$-module of finite length.

1. Each Lie algebra cohomology space $H^{p}(\overline{\mathfrak{u}}, X)$ is an $(\mathfrak{l}, L \cap K)$-module of finite length.

2. The irreducible $(\mathfrak{l}, L \cap K)$-module $J\left(\Gamma_{\mathfrak{q}}\right)$ can appear in $H^{p}(\overline{\mathfrak{u}}, X)$ only if

$$
p \geq s={ }_{\text {def }} \operatorname{dim} \overline{\mathfrak{u}} \cap \mathfrak{k} .
$$

3. The occurrence of $J\left(\Gamma_{\mathfrak{q}}\right)$ in Lie algebra cohomology $H^{s}(\overline{\mathfrak{u}}, X)$ corresponds to the occurrence of $X$ as a quotient of $I_{\text {quo }}(\Gamma)$ :

$$
\operatorname{Hom}_{\mathfrak{l}, L \cap K}\left(J_{\text {quo }}\left(\Gamma_{\mathfrak{q}}\right), H^{s}(\overline{\mathfrak{u}}, X)\right) \simeq \operatorname{Hom}_{\mathfrak{g}, K}\left(I_{\text {quo }}(\Gamma), X\right) .
$$

4. The coefficient $a_{X}(\Gamma)$ of $\Gamma$ in the numerator of the character formula for $X$ (5.12) is equal to the alternating sum over $p$ of the multiplicities of $J\left(\Gamma_{\mathfrak{q}}\right)$ in $H^{p}(\overline{\mathfrak{u}}, X)$. (Here the Weyl denominator function should be chosen using a positive root system containing the roots of $\overline{\mathfrak{u}}$.)

Sketch of proof. Part (1) is [38], Corollary 5.2.4. Part (2) is [38], Theorem 6.5.9(f) combined with Corollary 6.4.13. (To be precise, the references concern $\mathfrak{u}$ cohomology in degree $m-p$, with $m=\operatorname{dim} \mathfrak{u}$. One can deduce the statements needed here by a Poincaré duality relating $H^{p}(\overline{\mathfrak{u}}, X)^{h}$ (any of the Hermitian dual functors introduced in section (8.5) will do) to $H^{m-p}\left(\mathfrak{u}, X^{h} \otimes \wedge^{m}(\mathfrak{u})\right)$ ([21], Corollary III.3.6 and page 409). Alternatively, one can observe that the proof in [38] can be modified in a very straightforward way to give (2).) Part (3) is [38], Theorem 6.5.9(g) (with a parallel caveat about duality). Part (4) is [40], Theorem 8.1. 
Finally, it will be useful (for the unitarity algorithm) to have an explicit $\{$ se:VZLKT $\}$ description of the lowest $K$-types of standard modules. We begin as in (9.3) with a weak Langlands parameter

$$
\Gamma=(\Lambda, \nu)
$$

for a $\theta$-stable Cartan subgroup $H=T A$, and the Levi subgroup

$$
L=\text { centralizer in } G \text { of } T_{0} \supset H \text {, }
$$

corresponding to the real roots of $H$ in $G$. Now however we strengthen the hypothesis $(9.3 \mathrm{~b})$ to

$$
\left\langle d \Lambda, \alpha^{\vee}\right\rangle \geq 0 \quad(\alpha \in \Delta(\mathfrak{u}, \mathfrak{h})) ;
$$

we say that $\mathfrak{q}$ is of type $V Z L K T$ with respect to $\Gamma$, since in Theorem 9.7 below we will construct the lowest $K$-types of standard modules using Zuckerman's cohomological induction from $\mathfrak{q}$. Using this special choice of $\mathfrak{q}$, we construct the character

$$
\Gamma_{\mathfrak{q}}=\left(\Lambda_{\mathfrak{q}}, \nu\right) \in \widehat{T} \times \mathfrak{a}^{*}
$$

as in (9.3d) above. The Harish-Chandra modules for $L$ used in the construction of $I(\Gamma)$ in Theorem 9.4 satisfy

$$
\left.I_{\text {quo }}\left(\Gamma_{\mathfrak{q}}\right)\right|_{L \cap K}=\left.I_{\text {sub }}\left(\Gamma_{\mathfrak{q}}\right)\right|_{L \cap K}=\operatorname{Ind}_{T}^{L \cap K}\left(\Lambda_{\mathfrak{q}}\right) .
$$

Choose a maximal (connected) torus

$$
T_{f} \subset L \cap K .
$$

Necessarily $T_{f} \supset T_{0}$, and $T_{f}$ is a maximal torus in $K$. We will discuss representations of $K$ in using $T_{f}$-highest weights, bearing in mind that $K$ may be disconnected. We therefore choose a set of positive roots

$$
\Delta^{+}\left(\mathfrak{l} \cap \mathfrak{k}, \mathfrak{t}_{f}\right),
$$

and extend it to

$$
\Delta^{+}\left(\mathfrak{k}, \mathfrak{t}_{f}\right)={ }_{\operatorname{def}} \Delta^{+}\left(\mathfrak{l} \cap \mathfrak{k}, \mathfrak{t}_{f}\right) \cup \Delta\left(\mathfrak{u} \cap \mathfrak{k}, \mathfrak{t}_{f}\right) .
$$

Write

$$
\mathfrak{u}=\mathfrak{u} \cap \mathfrak{k} \oplus \mathfrak{u} \cap \mathfrak{s}
$$


for the decomposition of $\mathfrak{u}$ into the +1 and -1 eigenspaces of $\theta$. We will need the one-dimensional character

$$
2 \rho(\mathfrak{u}) \in \widehat{L}, \quad 2 \rho(\mathfrak{u})(\ell)=\operatorname{det}\left(\left.\operatorname{Ad}(\ell)\right|_{\mathfrak{u}}\right) .
$$

The restriction of this character to $L \cap K$ factors as

$$
\left.2 \rho(\mathfrak{u})\right|_{L \cap K}=2 \rho(\mathfrak{u} \cap \mathfrak{k})+2 \rho(\mathfrak{u} \cap \mathfrak{s}) .
$$

Theorem 9.7 (Cohomological induction of lowest $K$-types; [21], Theorem $\{\mathrm{thm}: \mathrm{VZLKT}\}$ 10.44). Suppose $\Gamma=(\Lambda, \nu)$ is a weak Langlands parameter, and $\mathfrak{q}=\mathfrak{l}+\mathfrak{u}$ is a $\theta$-stable parabolic subalgebra of type VZLKT (see (9.6c)). Use the notation of (9.6) above.

List the lowest $L \cap K$-types of

$$
\left.I\left(\Gamma_{\mathfrak{q}}\right)\right|_{L \cap K}=\operatorname{Ind}_{T}^{L \cap K}\left(\Lambda_{\mathfrak{q}}\right)
$$

as

$$
\mu_{1}^{L \cap K}, \mu_{2}^{L \cap K}, \ldots, \mu_{r}^{L \cap K} \in \widehat{L \cap K} .
$$

For each $\mu_{i}^{L \cap K}$, choose a highest $T_{f}$-weight

$$
\phi_{i}^{L \cap K} \in \widehat{T_{f}} .
$$

Define

$$
\phi_{i}=\phi_{i}^{L \cap K}+2 \rho(\mathfrak{u} \cap \mathfrak{s}) \in \widehat{T_{f}} .
$$

1. For each $i$, the representation of $K$

$$
\mu_{i}=\left(\Pi_{L \cap K}^{K}\right)_{s}\left[U(\mathfrak{k}) \otimes_{\overline{\mathfrak{q}} \cap \mathfrak{k}}\left(\mu_{i}^{L \cap K} \otimes 2 \rho(\mathfrak{u})\right)\right]
$$

is irreducible or zero, depending on whether or not the weight $\phi_{i}$ is dominant for $\Delta^{+}\left(\mathfrak{k}, \mathfrak{t}_{f}\right)$.

2. In the dominant case, $\phi_{i}$ is a highest weight of $\mu_{i}$, and $\mu_{i}$ may be characterized as the unique irreducible representation of $K$ containing $\mathfrak{u} \cap \mathfrak{k}$-invariant vectors transforming under the representation

$$
\mu_{i}^{L \cap K} \otimes 2 \rho(\mathfrak{u} \cap \mathfrak{s}) \in \widehat{L \cap K} .
$$

Briefly, we say that $\mu_{i}$ has "highest weight $\mu_{i}^{L \cap K} \otimes 2 \rho(\mathfrak{u} \cap \mathfrak{s})$."

3. The irreducible representations $\mu_{i}$ constructed in (1) exhaust the lowest $K$-types of $I_{\mathrm{quo}}(\Gamma)$, by means of the (K-equivariant) bottom layer map

$$
\mu_{i} \rightarrow I_{\text {quo }}(\Gamma)
$$

evident from the realization in Theorem 9.4. 
4. Suppose that $\Lambda$ is final (Definition 6.5; that is, that $\Lambda_{\mathfrak{q}}\left(m_{\alpha}\right)=1$ for every real root $\alpha)$. Then there is a unique lowest $L \cap K$-type $\mu_{1}^{L \cap K}$ of $\operatorname{Ind}_{T}^{L \cap K}\left(\Lambda_{\mathfrak{q}}\right)$. It has dimension one, and may be characterized by the two requirements

$$
\left.\mu_{1}^{L \cap K}\right|_{T}=\Lambda_{\mathfrak{q}},\left.\quad \mu_{1}^{L \cap K}\right|_{\left[L_{0}, L_{0}\right] \cap K} \text { is trivial. }
$$

The (unique) highest weight of $\mu_{1}^{L \cap K}$ may be characterized by

$$
\left.\phi_{1}^{L \cap K}\right|_{T \cap T_{f}}=\left.\Lambda_{\mathfrak{q}}\right|_{T \cap T_{f}}, \quad\left\langle\phi_{1}^{L \cap K}, \alpha^{\vee}\right\rangle=0 \quad\left(\alpha \in \Delta\left(\mathfrak{l}, \mathfrak{t}_{f}\right)\right),
$$

Sketch of proof. Parts (1)-(3) are stated as Theorem 10.44 in [21]. Unfortunately that reference does not provide a complete proof; but what is needed are just the facts established in [38], section 6.5. For (4), the "final" hypothesis means exactly that $\Lambda_{\mathfrak{q}}$ is trivial on $\left[L_{0}, L_{0}\right] \cap T$, which is generated by the elements $m_{\alpha}$ for real roots $\alpha$. It follows that any lowest $L \cap K$-type must be trivial on $\left[L_{0}, L_{0}\right] \cap K$. Because (as one easily checks) $L \cap K$ is generated by $T$ and $\left[L_{0}, L_{0}\right] \cap K$, the remaining assertions in (4) follow immediately.

\section{Invariant forms on irreducible representations}

Suppose $G$ is the group of real points of a connected complex reductive algebraic group, $K$ is a maximal compact subgroup of $G$, and $\theta$ is a Cartan involution (notation as in (3.1) and Theorem 3.4). Write

$$
\begin{gathered}
\mathfrak{g}=\operatorname{Lie}(G) \otimes_{\mathbb{R}} \mathbb{C}, \\
K(\mathbb{C})=\text { complexification of } K,
\end{gathered}
$$

so that $(\mathfrak{g}, K(\mathbb{C}))$ is a pair (Definition 8.1). We are interested in two real structures (Definition 8.1) on this pair: the classical real structure $\sigma_{0}$ given by the real form $G$, and the real structure $\sigma_{c}$ given by the compact real form

$$
\sigma_{c}=\sigma_{0} \circ \theta
$$

(Theorem 3.4). These two structures are the same on $K(\mathbb{C})$ (where they both define the compact form $K$ ), and therefore they agree on $\mathfrak{k}$; but they differ (by a factor of -1 ) on the -1 eigenspace $\mathfrak{s}$ of $\theta$ on $\mathfrak{g}$ (see Theorem 3.4). Suppose now that $V$ is a $(\mathfrak{g}, K(\mathbb{C}))$-module. Classically, an invariant Hermitian form on $V$ is a Hermitian form $\langle,\rangle^{0}$ on $V$ with the properties

$$
\begin{aligned}
\langle k \cdot v, w\rangle^{0}=\left\langle v, k^{-1} \cdot w\right\rangle^{0}, & \langle X \cdot v, w\rangle^{0}=-\langle v, X \cdot w\rangle^{0} \\
& \left(k \in K, X \in \mathfrak{g}_{0}, v, w \in V\right) .
\end{aligned}
$$


(This is the definition that we left unstated in Theorem 7.1.) The first requirement is that the compact group $K$ act by "unitary" (that is, preserving the Hermitian form) operators; and the second is that the real Lie algebra $\mathfrak{g}_{0}$ act by skew-Hermitian operators. This in turn is the differentiated version of the condition that $G$ act by unitary operators. The assumption that $\mathfrak{k}_{0}$ acts by skew-Hermitian operators guarantees that $K_{0}$ acts by unitary operators; so we need the first hypothesis only for a single element $k$ in each connected component of $K$.

It is equivalent to require either less

$$
\begin{aligned}
\langle k \cdot v, w\rangle^{0}=\left\langle v, k^{-1} \cdot w\right\rangle^{0}, & \langle X \cdot v, w\rangle^{0}=-\langle v, X \cdot w\rangle^{0} \\
& \left(k \in K, X \in \mathfrak{s}_{0}, v, w \in V\right)
\end{aligned}
$$

or more

$$
\begin{aligned}
\langle k \cdot v, w\rangle^{0}=\left\langle v, \sigma_{0}(k)^{-1} \cdot w\right\rangle^{0}, \quad & \langle X \cdot v, w\rangle^{0}=-\left\langle v, \sigma_{0}(X) \cdot w\right\rangle^{0} \\
& (k \in K(\mathbb{C}), X \in \mathfrak{g}, v, w \in V)
\end{aligned}
$$

Of course these are precisely the properties defining a $\sigma_{0}$-invariant Hermitian form on $V$ (see (8.7)).

In Section 8 we related the existence of invariant forms to the notion of Hermitian dual of any $(\mathfrak{g}, K(\mathbb{C}))$-module. We therefore need to compute Hermitian duals in terms of the Langlands classification.

Definition 10.3. Suppose $\Gamma=\left(H, \gamma, R_{i \mathbb{R}}^{+}\right)$is a Langlands parameter (Theorem 6.1). The Hermitian dual of $\Gamma$ is

$$
\Gamma^{h, \sigma_{0}}=\left(H,-\bar{\gamma}, R_{i \mathbb{R}}^{+}\right) .
$$

As usual the additive notation for characters may be a bit confusing; the character of the $\rho_{i \mathbb{R}}$ cover $\widetilde{H}$ is

$$
(-\bar{\gamma})(\widetilde{h})=_{\operatorname{def}} \overline{\gamma\left(\widetilde{h}^{-1}\right)}
$$

Now write $H=T A$ as in 4.3 , and

$$
\Gamma=(\Lambda, \nu)=\left(\Lambda, \nu_{\mathrm{re}}+i \nu_{\mathrm{im}}\right)
$$

as in 6.5 , with $\Lambda$ a discrete Langlands parameter and $\nu \in \mathfrak{a}^{*}$. Since $\gamma$ takes values in the unit circle on the maximal compact subgroup $\widetilde{T}$ of $\widetilde{H}$, we find

$$
\Gamma^{h, \sigma_{0}}=(\Lambda,-\bar{\nu})=(\Lambda, \theta \bar{\nu})=\left(\Lambda,-\nu_{\mathrm{re}}+i \nu_{\mathrm{im}}\right) .
$$

\{eq: dualform $\}$ 
The most important feature of this formula is that if the continuous parameter $\nu$ is imaginary (Definition 6.5, corresponding to a unitary character of $A$ ) then $\Gamma^{h, \sigma_{0}}$ is equal to $\Gamma$. We will see (Proposition 10.4) that this means that the corresponding representation admits an invariant Hermitian form. These are the tempered irreducible representations of $G$, each of which has a natural Hilbert space realization, and therefore a positive-definite invariant Hermitian form.

Proposition 10.4 (Knapp-Zuckerman; see [19], Chapter 16). Suppose $\Gamma=$ \{prop:dualstd $(\Lambda, \nu)$ is a Langlands parameter for the real reductive group $G$, and $\Gamma^{h, \sigma_{0}}=$ $(\Lambda,-\bar{\nu})$ is the Hermitian dual parameter.

1. The Hermitian dual of the standard representation with a Langlands quotient is the standard representation with a Langlands submodule:

$$
\left[I_{\text {quo }}(\Gamma)\right]^{h, \sigma_{0}} \simeq I_{\text {sub }}\left(\Gamma^{h, \sigma_{0}}\right),
$$

and similarly with quotient and submodule reversed.

2. The Hermitian dual of the irreducible module $J(\Gamma)$ is

$$
[J(\Gamma)]^{h, \sigma_{0}} \simeq J\left(\Gamma^{h, \sigma_{0}}\right) .
$$

3. The irreducible module $J(\Gamma)$ admits a nonzero invariant Hermitian form if and only if the Langlands parameter $\Gamma$ is equivalent to $\Gamma^{h, \sigma_{0}}$. In this case there is a nonzero invariant Hermitian form on $I_{\mathrm{quo}}(\Gamma)$, unique up to a real scalar. This form has radical equal to the maximal proper submodule $I_{1}(\Gamma)$, and factors to a nondegenerate invariant Hermitian form on $J(\Gamma)$.

4. Write the parameter $\Gamma$ as

$$
\Gamma=(\Lambda, \nu)
$$

as in 6.5. Then $J(\Gamma)$ admits a nonzero invariant Hermitian form if and only if there is an element $w \in W^{\Lambda}$ (Proposition 6.6) such that $w \cdot \nu=-\bar{\nu}$. (If such an element $w$ exists, it may be chosen to have order 2.)

5. If $J(\Gamma)$ admits a nonzero invariant Hermitian form, then this form is unique up to a nonzero real scalar multiple. Consequently the signature function

$$
\left(\operatorname{pos}_{J(\Gamma)}^{\sigma_{0}}, \operatorname{neg}_{J(\Gamma)}^{\sigma_{0}}\right): \widehat{K} \rightarrow \mathbb{N} \times \mathbb{N}
$$

(cf. Proposition 8.9) is uniquely defined up to interchanging the two factors pos and neg. 
We will not reproduce the proof in detail, but here is an outline. Recall the description in (6.4) and Theorem 9.2 of the standard module $I_{\text {quo }}(\Gamma)$ as induced from a discrete series representation; that is, as a space of functions $f_{\text {quo }}$ on $G$ with values in the discrete series representation $D(\Lambda)$, transforming appropriately under right translation by a parabolic subgroup $P$. What we have proposed as the Hermitian dual is a space of functions $f_{\text {sub }}$ on $G$ with values in this same discrete series representation, having a slightly different transformation law under $P$. Using the inner product on the (unitary) discrete series representation gives a complex-valued function $\left\langle f_{\text {quo }}(g), f_{\text {sub }}(g)\right\rangle$, which transforms under $P$ exactly by the modular character of $P$. The inner product function may therefore be identified with a measure on the compact homogeneous space $G / P$, and integrated; then

$$
\left\langle f_{\text {quo }}, f_{\text {sub }}\right\rangle=\int_{G / P}\left\langle f_{\text {quo }}(g), f_{\text {sub }}(g)\right\rangle
$$

is the invariant Hermitian pairing we want between $I_{\text {quo }}(\Gamma)$ and $I_{\text {sub }}(\Gamma)$.

Having recalled the classical theory of invariant Hermitian forms, we now turn to our main technical tool: the notion of $c$-invariant Hermitian forms.

Suppose again that $V$ is a $(\mathfrak{g}, K(\mathbb{C}))$-module. A $c$-invariant Hermitian form on $V$ is a Hermitian form $\langle,\rangle^{c}$ on $V$ with the properties

$$
\begin{aligned}
\langle k \cdot v, w\rangle^{c}=\left\langle v, k^{-1} \cdot w\right\rangle^{c}, & \langle X \cdot v, w\rangle^{c}=-\langle v, X \cdot w\rangle^{c} \\
& \left(k \in K, X \in \mathfrak{g}^{\sigma_{c}}, v, w \in V\right) .
\end{aligned}
$$

Just as for a classical Hermitian form, we require first that the compact group $K$ act by unitary (that is, preserving the Hermitian form) operators. What is new is that we require not the real Lie algebra $\mathfrak{g}_{0}=\mathfrak{g}^{\sigma_{0}}$, but rather the compact Lie algebra $\mathfrak{g}^{\sigma_{c}}$, to act by skew-Hermitian operators. Because the action of $\mathfrak{g}^{\sigma_{c}}$ usually does not exponentiate to the compact real form of $G$, this new requirement is no longer the derivative of a unitarity requirement on a group. Nevertheless the definition still makes sense.

It is equivalent to require either less

$$
\begin{aligned}
\langle k \cdot v, w\rangle^{c}=\left\langle v, k^{-1} \cdot w\right\rangle^{c}, & \langle X \cdot v, w\rangle^{c}=-\langle v, X \cdot w\rangle^{c} \\
& \left(k \in K, X \in i \mathfrak{s}_{0}, v, w \in V\right)
\end{aligned}
$$

or more

$$
\begin{array}{r}
\langle k \cdot v, w\rangle^{c}=\left\langle v, \sigma_{c}(k)^{-1} \cdot w\right\rangle^{c}, \quad \\
\langle X \cdot v, w\rangle^{c}=-\left\langle v, \sigma_{c}(X) \cdot w\right\rangle^{c} \\
(k \in K(\mathbb{C}), X \in \mathfrak{g}, v, w \in V)
\end{array}
$$


These are precisely the properties defining a $\sigma_{c}$-invariant Hermitian form on $V$ (see Definition 8.6).

We need to compute $c$-Hermitian duals in terms of the Langlands classification.

Definition 10.6. Suppose $\Gamma=\left(H, \gamma, R_{i \mathbb{R}}^{+}\right)$is a Langlands parameter (Theorem 6.1). The c-Hermitian dual of $\Gamma$ is

$$
\Gamma^{h, \sigma_{c}}=\left(H,-\bar{\gamma} \circ \theta, R_{i \mathbb{R}}^{+}\right) .
$$

This is the same as the Hermitian dual, except that we have twisted the character by the Cartan involution $\theta$. Write $H=T A$ as in 4.3 , and

$$
\Gamma=(\Lambda, \nu)=\left(\Lambda, \nu_{\mathrm{re}}+i \nu_{\mathrm{im}}\right)
$$

as in 6.5 , with $\Lambda$ a discrete Langlands parameter and $\nu \in \mathfrak{a}^{*}$. Since $\theta$ acts by the identity on $T$ and by inversion on $A$, comparison with Definition 10.3 shows that

$$
\Gamma^{h, \sigma_{c}}=(\Lambda, \bar{\nu})=\left(\Lambda, \nu_{\mathrm{re}}-i \nu_{\mathrm{im}}\right) .
$$

$\{$ eq: cdualform $\}$

In particular, if the continuous parameter of $\Gamma$ is real (Definition 6.5) then $\Gamma^{h, \sigma_{c}}$ is equal to $\Gamma$. We will see (Proposition 10.7) that this means that the corresponding representation admits a $c$-invariant Hermitian form.

Proposition 10.7. Suppose $\Gamma=(\Lambda, \nu)$ is a Langlands parameter for the real reductive group $G$, and $\Gamma^{h, \sigma_{c}}=(\Lambda, \bar{\nu})$ is the c-Hermitian dual parameter.

1. The c-Hermitian dual of the standard representation with a Langlands quotient is a standard representation with a Langlands submodule:

$$
\left[I_{\mathrm{quo}}(\Gamma)\right]^{h, \sigma_{c}} \simeq I_{\mathrm{sub}}\left(\Gamma^{h, \sigma_{c}}\right),
$$

and similarly with quotient and submodule reversed.

2. The c-Hermitian dual of the irreducible module $J(\Gamma)$ is

$$
[J(\Gamma)]^{h, \sigma_{c}} \simeq J\left(\Gamma^{h, \sigma_{c}}\right) .
$$

3. The irreducible module $J(\Gamma)$ admits a nonzero c-invariant Hermitian form if and only if the Langlands parameter $\Gamma$ is equivalent to $\Gamma^{h, \sigma_{c}}$. In this case there is a nonzero c-invariant Hermitian form on $I_{\mathrm{quo}}(\Gamma)$, unique up to a real scalar. This form has radical equal to the maximal proper submodule $I_{1}(\Gamma)$, and factors to a nondegenerate c-invariant Hermitian form on $J(\Gamma)$. 
4. Write the parameter $\Gamma$ as

$$
\Gamma=(\Lambda, \nu)
$$

as in 6.5. Then $J(\Gamma)$ admits a nonzero c-invariant Hermitian form if and only if there is an element $w \in W^{\Lambda}$ (Proposition 6.6) such that $w \cdot \nu=\bar{\nu}$. In particular, if $\nu$ is real, then a c-invariant form always exists.

5. Suppose that the continuous parameter $\nu$ is real. Then any c-invariant Hermitian form on $J(\Gamma)$ has the same sign on every lowest $K$-type. In particular, the form may be chosen to be positive definite on every lowest $K$-type, and is characterized up to a positive real scalar multiple by this requirement. Consequently the signature function

$$
\left(\operatorname{pos}_{J(\Gamma)}^{c}, \operatorname{neg}_{J(\Gamma)}^{c}\right): \widehat{K} \rightarrow \mathbb{N} \times \mathbb{N}
$$

(cf. Proposition 8.9) is uniquely defined by the characteristic property

$$
\left(\operatorname{pos}_{J(\Gamma)}^{c}(\mu), \operatorname{neg}_{J(\Gamma)}^{c}(\mu)\right)=(1,0) \quad \mu \text { any lowest } K \text {-type of } J(\Gamma) .
$$

Proof. We use the proof of Proposition 10.4. Knapp and Zuckerman began \{se:cdualproof $\}$ with the realization

$$
I_{\text {quo }}(\Gamma)=\operatorname{Ind}_{M A N}^{G}(D(\Lambda) \otimes \nu \otimes 1)
$$

(with $P$ chosen of type $\mathrm{L}$ with respect to $\Gamma$ ) and found a natural isomorphism

$$
\left[I_{\text {quo }}(\Gamma)\right]^{h, \sigma_{0}} \simeq \operatorname{Ind}_{M A N}^{G}(D(\Lambda) \otimes-\bar{\nu} \otimes 1) .
$$

Because of the minus sign on the right, it is easy to check that $P^{\text {op }}$ is of type $\mathrm{L}$ with respect to $\Gamma^{h, \sigma_{0}}$; so what appears on the right is the realization of $I_{\text {sub }}\left(\Gamma^{h, \sigma_{0}}\right)$ from Theorem 9.2. For the $c$-Hermitian dual, we are again looking at the (same) Hermitian dual of the space of the standard module; what has changed is that the representation of $\mathfrak{g}$ on this dual space has been twisted by the Cartan involution $\theta$. Now $\theta$ carries $P=M A N$ to $P^{\mathrm{op}}=M A N^{\mathrm{op}}$, so it is clear that (writing a superscript for the operation of twisting the Lie algebra action by an automorphism)

$$
\left[\operatorname{Ind}_{M A N}^{G}(D(\Lambda) \otimes-\bar{\nu} \otimes 1)\right]^{\theta} \simeq \operatorname{Ind}_{M A N^{\mathrm{op}}}^{G}\left(D(\Lambda)^{\theta} \otimes \bar{\nu} \otimes 1\right)
$$

Harish-Chandra characterized each discrete series representation in terms of its distribution character on the $\theta$-fixed subgroup $K$, so $D(\Lambda)^{\theta} \simeq D(\Lambda)$ 
whenever $\Lambda$ is a discrete series representation. The construction of limits of discrete series by the translation principle inherits this property. Assembling all of this, we find

$$
\left[I_{\mathrm{quo}}(\Gamma)\right]^{h, \sigma_{c}} \simeq \operatorname{Ind}_{M A N^{\mathrm{op}}}^{G}(D(\Lambda) \otimes \bar{\nu} \otimes 1) .
$$

Because the minus sign on $\nu$ has disappeared, it is easy to check that $P$ is of type $\mathrm{L}$ with respect to $\Gamma^{h, \sigma_{c}}$. According to Theorem 9.2, the right side realizes $I_{\text {sub }}\left(\Gamma^{h, \sigma_{c}}\right)$. This proves $(1)$.

Parts (2) and (3) are a formal consequence of (1) and the Langlands classification. Part (4) is a restatement of (3), using Proposition 6.6.

For (5), we will use

Theorem 10.9 (Signature Theorem; [21], Theorem 6.34). Suppose $\mathfrak{q}=$ $\mathfrak{l}+\mathfrak{u}$ is a $\theta$-stable parabolic subalgebra of $\mathfrak{g}$, with corresponding Levi subgroup $L \subset G$. Write $s=\operatorname{dim}(\mathfrak{u} \cap \mathfrak{k})$, and write $\mathcal{L}_{s}$ for Zuckerman's cohomological parabolic induction functor from $(\mathfrak{l}, L \cap K)$-modules to $(\mathfrak{g}, K)$-modules ([21], pages 327-328).

1. Suppose that $Z$ is an $(\mathfrak{l}, L \cap K)$-module of finite length admitting an invariant Hermitian form $\langle,\rangle_{L}^{0}$. Then there is an induced invariant Hermitian form $\langle,\rangle_{G}^{0}$ on $\mathcal{L}_{s}(Z)$. The signature of $\langle,\rangle_{G}^{0}$ on the bottom layer of $K$-types matches the signature of $\langle,\rangle_{L}^{0}$ on the corresponding $L \cap K$-types.

2. Suppose that $Z$ is an $(\mathfrak{l}, L \cap K)$-module of finite length admitting a $c$ invariant Hermitian form $\langle,\rangle_{L}^{c}$. Then there is an induced c-invariant Hermitian form $\langle,\rangle_{G}^{c}$ on $\mathcal{L}_{s}(Z)$. The signature of $\langle,\rangle_{G}^{c}$ on the bottom layer of $K$-types matches the signature of $\langle,\rangle_{L}^{c}$ on the corresponding $L \cap K$-types.

Proof. Part (1) is Theorem 6.34 of [21]. Part (2) can be proved by repeating almost exactly the same words, replacing "invariant Hermitian" by "c-invariant Hermitian."

We apply Theorem 10.9 to the realization of the standard module $I(\Gamma)$ in Theorem 9.4, and to the description of the lowest $K$-types in Theorem 9.7. What this accomplishes is a reduction of (5) to the special case

$$
H \text { is split modulo center of } G \text {; }
$$

that is, that all roots of $H$ are real, so that

$$
P=M A N=T A N
$$


is a Borel subgroup of $G$, and the standard representations are ordinary principal series representations. We will assume that $P$ is chosen to make $\nu=\operatorname{Re} \nu$ weakly dominant:

$$
\left\langle\nu, \alpha^{\vee}\right\rangle \geq 0 \quad(\alpha \in \Delta(\mathfrak{n}, \mathfrak{h})
$$

this is a stronger hypothesis than "type L" (9.1d). Consider the parabolic subgroup $P_{1}=M_{1} A_{1} N_{1} \supset P$ defined by either of the equivalent requirements

$$
\left\langle\nu, \alpha^{\vee}\right\rangle=0 \quad\left(\alpha \in \Delta\left(\mathfrak{m}_{1}, \mathfrak{h}\right),\right.
$$

or

$$
\left\langle\nu, \alpha^{\vee}\right\rangle>0 \quad\left(\alpha \in \Delta\left(\mathfrak{n}_{1}, \mathfrak{h}\right)\right)
$$

Define

$$
H_{1}=M_{1} \cap H, \quad \Gamma_{1}=\left.\Gamma\right|_{H_{1}}, \quad \nu_{1}=\nu \mid A_{1}, \quad I_{1}=I\left(\Gamma_{1}\right) ;
$$

this last representation is a tempered unitary principal series representation of $M_{1}$, with continuous parameter equal to zero. Induction by stages says

$$
I_{\text {quo }}(\Gamma)=\operatorname{Ind}_{M_{1} A_{1} N_{1}}^{G}\left(I_{1} \otimes \nu_{1} \otimes 1\right) .
$$

Here finally we have written the standard module precisely as Langlands wrote it in [24]: as induced from a tempered representation $I_{1}$ twisted by a character $\nu$ with $\operatorname{Re} \nu$ strictly positive. Because $\Gamma$ is assumed to be a Langlands parameter, this standard module has a unique irreducible quotient $J(\Gamma)$. It follows that the unitary representation $I_{1}$ must be irreducible. A principal series representation with continuous parameter equal to zero is irreducible if and only if it has a unique lowest $K$ type; so we conclude that

$$
I_{1} \text { is irreducible, with unique lowest } M_{1} \cap K \text {-type } \mu_{1} \text {. }
$$

We may therefore fix a nondegenerate $c$-invariant Hermitian form $\langle,\rangle_{1}$ on $I_{1}$ with the property that

$\langle,\rangle_{1}$ is positive definite on the lowest $M_{1} \cap K$-type $\mu_{1}$;

this determines the form up to a positive scalar multiple.

The $c$-hermitian dual of $I_{\text {quo }}(\Gamma)$ is

$$
\left[I_{\mathrm{quo}}(\Gamma)\right]^{h, \sigma_{c}}=\operatorname{Ind}_{M_{1} A_{1} N_{1}^{\mathrm{op}}}^{G}\left(I_{1}^{h, \sigma_{c}} \otimes \nu_{1} \otimes 1\right) \simeq \operatorname{Ind}_{M_{1} A_{1} N_{1}^{\mathrm{op}}}^{G}\left(I_{1} \otimes \nu_{1} \otimes 1\right) .
$$


The last identification here uses the pairing $\langle,\rangle_{1}$ just defined.

A vector in $I_{\text {quo }}(\Gamma)$ is a function $f_{\text {quo }}$ on $G$ with values in $I_{1}$, transforming in a certain way under the action of $P_{1}$ on the right. A vector in the $c$ Hermitian dual is a function $f_{\text {sub }}$ on $G$, taking values in $I_{1}$, and transforming under the action of $P_{1}^{\text {op }}$ on the right. The pairing between two such functions is

$$
\int_{K}\left\langle f_{\mathrm{quo}}(k), f_{\mathrm{sub}}(k)\right\rangle_{1} d k .
$$

A $c$-invariant Hermitian pairing on $I_{\text {quo }}(\Gamma)$ is therefore the same thing as an intertwining operator

$$
\mathcal{L}: \operatorname{Ind}_{M_{1} A_{1} N_{1}}^{G}\left(I_{1} \otimes \nu_{1} \otimes 1\right) \rightarrow \operatorname{Ind}_{M_{1} A_{1} N_{1}^{\text {op }}}^{G}\left(I_{1} \otimes \nu_{1} \otimes 1\right) ;
$$

the corresponding $c$-invariant Hermitian form is

$$
\left\langle f_{\text {quo }}, f_{\text {quo }}^{\prime}\right\rangle_{\mathcal{L}}=\int_{K}\left\langle f_{\text {quo }}(k),\left(\mathcal{L} f_{\text {quo }}^{\prime}\right)(k)\right\rangle_{1} d k
$$

$\{$ eq: cinvtsplit $\}$

In this formula we can use the integral long intertwining operator

$$
(\mathcal{L} f)(g)=\int_{N_{1}^{\text {op }}} f(g x) d x
$$

this integral over $N_{1}^{\mathrm{op}}$ is absolutely convergent because $\operatorname{Re} \nu_{1}$ is strictly positive on the roots of $A_{1}$, just as in [24].

The lowest $K$-types of $I_{\text {quo }}(\Gamma)$ are the fine representations of $K$ ([38], Definition 4.3.9) that contain the (necessarily fine) representation $\mu_{1}$ of $M_{1} \cap$ $K$. Each of them contains $\mu_{1}$ with multiplicity one ([38], Theorem 4.3.16), so each lowest $K$-type $\mu$ has multiplicity one in $I_{\text {quo }}(\Gamma)$. Consequently

$\mathcal{L}$ acts by a scalar $\mathcal{L}(\mu)$ on each lowest $K$-type $\mu$.

In light of the formula $(10.10 \mathrm{~m})$ for the $c$-invariant form, and the positivity (10.10i), the proof of (5) comes down to proving

$$
\begin{aligned}
& \mathcal{L} \text { acts by a strictly positive scalar on each } \\
& \text { fine representation } \mu \text { containing } \mu_{1} \text {. }
\end{aligned}
$$

In order to compute the scalar $\mathcal{L}(\mu)$, we use the product formula of Gindikin-Karpelevič (see Schiffmann [31] or [38], Theorem 4.2.22) for the intertwining operator $\mathcal{L}$. What this product formula shows is that the scalar in question is a product (over the coroots of $H$ taking strictly positive values on $\nu$ ) of a corresponding scalar computed in the three-dimensional subgroup 
$\phi_{\alpha}(S L(2, \mathbb{R}))$. There are two cases: the "even case," in which the character $\delta$ is trivial on the element $m_{\alpha}$ of order two (Definition 5.7); and the "odd case," in which $\delta\left(m_{\alpha}\right)=-1$.

In the even case, the only fine representation of $S O(2)$ containing $\delta$ is the trivial one, so we must begin with the constant function 1 on $S O(2)$ and extend it to an element of the principal series representation

$$
f_{\text {even }}\left(\left(\begin{array}{cc}
\cos \theta & \sin \theta \\
-\sin \theta & \cos \theta
\end{array}\right)\left(\begin{array}{cc}
e^{t} & 0 \\
0 & e^{-t}
\end{array}\right)\left(\begin{array}{cc}
1 & x \\
0 & 1
\end{array}\right)\right)=e^{-t(v+1)} .
$$

Here $v=\left\langle\nu, \alpha^{\vee}\right\rangle$ is a strictly positive parameter. Necessarily $\mathcal{L}\left(f_{\text {even }}\right)$ is constant on $S O(2)$. To compute the constant, we can evaluate at 1 , getting

$$
\int_{-\infty}^{\infty} f_{\text {even }}\left(\left(\begin{array}{ll}
1 & 0 \\
y & 1
\end{array}\right)\right) d y=\int_{-\infty}^{\infty}\left(1+y^{2}\right)^{-(v+1) / 2} d y
$$

(Here we have computed the Iwasawa decomposition of the matrix in the integrand, then used the formula above.) Convergence and positivity of this integral are elementary. (Explicit evaluation, to $\pi^{1 / 2} \Gamma(v / 2) / \Gamma(v / 2+1 / 2)$, is also easy and well known, but we will not need this.)

In the odd case, there are two fine representations of $S O(2)$ containing $\delta$, corresponding to the functions $e^{ \pm i \theta}$ on $S O(2)$. The corresponding functions in the principal series are

$$
f_{\text {odd }, \pm}\left(\left(\begin{array}{cc}
\cos \theta & \sin \theta \\
-\sin \theta & \cos \theta
\end{array}\right)\left(\begin{array}{cc}
e^{t} & 0 \\
0 & e^{-t}
\end{array}\right)\left(\begin{array}{ll}
1 & x \\
0 & 1
\end{array}\right)\right)=e^{ \pm i \theta} e^{-t(v+1)}
$$

Again applying $\mathcal{L}$ has to give a multiple of $e^{ \pm i \theta}$ on $S O(2)$, and we can calculate the multiple by evaluating at the identity:

$$
\int_{-\infty}^{\infty} f_{\text {odd }, \pm}\left(\left(\begin{array}{ll}
1 & 0 \\
y & 1
\end{array}\right)\right) d y=\int_{-\infty}^{\infty}\left[(1 \mp i y)\left(1+y^{2}\right)^{-1 / 2}\right]\left(1+y^{2}\right)^{-(v+1) / 2} d y
$$

The imaginary part of the integrand is (integrable and) odd, so contributes zero; we are left with

$$
\int_{-\infty}^{\infty}\left(1+y^{2}\right)^{-(v+2) / 2} d y
$$

which is clearly positive. (The value is $\pi^{1 / 2} \Gamma(v / 2+1 / 2) / \Gamma(v / 2+1)$.) 


\section{Standard and c-invariant forms in the equal rank case}

$\{$ sec: eqrk\}

Our plan (roughly speaking) is (first) to calculate signatures of $c$-invariant forms, and (second) to relate these to the signatures of ordinary invariant forms that we care most about. The first step will occupy most of the rest of the paper. The second step is in most cases much easier, and we can do it now. But in some cases this easy step will fail. In order to repair it, we will introduce (in Section 12) a slightly different category of representations.

Definition 11.1. Suppose that $G$ is a real reductive algebraic group as in (3.1), and that $\theta$ is a Cartan involution as in Theorem 3.4, with $K=G^{\theta}$. We say that $G$ is equal rank if $G$ and $K$ have the same rank; equivalently, if the automorphism $\theta$ of $G$ is inner. In this case a strong involution for $G$ is an element $x \in G$ such that

$$
\operatorname{Ad}(x)=\theta, \quad K=G^{x}
$$

It follows that $x \in Z(K)$, and that

$$
x^{2}=z \in Z(G) \cap K .
$$

Fix $x$ and $z$ as above. For every $\zeta \in \mathbb{C}^{\times}$, define

$$
\widehat{G}_{\zeta}=\{V \in \widehat{G} \mid z \cdot v=\zeta v \quad(v \in V)\},
$$

the set of irreducible $(\mathfrak{g}, K(\mathbb{C}))$-modules in which $z$ acts by $\zeta$. Similarly define $\widehat{K}_{\zeta}$.

Fix a square root $\zeta^{1 / 2}$ of $\zeta$. On every $\mu \in \widehat{K}_{\zeta}, x$ must act by some square root of $\zeta$; so there is a sign

$$
\epsilon(\mu) \in\{ \pm 1\}, \quad \mu(x)=\epsilon(\mu) \zeta^{1 / 2} \quad\left(\mu \in \widehat{K}_{\zeta}\right) .
$$

Of course $\epsilon(\mu)$ depends on the choice of square root $\zeta^{1 / 2}$.

Theorem 11.2. Suppose $G$ is an equal rank real reductive algebraic group with strong involution $x \in Z(K)$ (Definition 11.1). Write $z=x^{2} \in Z(G)$. Suppose $J$ is an irreducible $(\mathfrak{g}, K(\mathbb{C}))$-module admitting a c-invariant Hermitian form $\langle,\rangle_{J}^{c}$, in which $z$ acts by the scalar $\zeta \in \mathbb{C}^{\times}$. Fix a square root $\zeta^{1 / 2}$ of $\zeta$. Then $J$ admits an invariant Hermitian form $\langle,\rangle_{J}^{0}$ defined by the formula

$$
\langle v, w\rangle_{J}^{0}=\zeta^{-1 / 2}\langle x \cdot v, w\rangle_{J}^{c}
$$


For each $\mu \in \widehat{K}_{\zeta}$, the forms on the $\mu$ multiplicity spaces (Proposition 8.9) are therefore related by

$$
\langle,\rangle_{J^{\mu}}^{0}=\epsilon(\mu)\langle,\rangle_{J^{\mu}}^{c}
$$

the sign $\epsilon(\mu)= \pm 1$ is defined in Definition 11.1. Consequently the signature functions are related by

$$
\left(\operatorname{pos}_{J}^{0}(\mu), \operatorname{neg}_{J}^{0}(\mu)\right)= \begin{cases}\left(\operatorname{pos}_{J}^{c}(\mu), \operatorname{neg}_{J}^{c}(\mu)\right) & (\epsilon(\mu)=+1) \\ \left(\operatorname{neg}_{J}^{c}(\mu), \operatorname{pos}_{J}^{c}(\mu)\right) & (\epsilon(\mu)=-1)\end{cases}
$$

Proof. The formula for the invariant Hermitian form is a special case of Proposition 8.16; the two automorphisms $\delta$ and $\epsilon$ of that proposition are both $\theta$, and we can choose $\lambda=x$. The statements about signatures are immediate.

Theorem 11.2 provides a very complete, explicit, and computable passage from the signature of a $c$-invariant Hermitian form to the signature of a classical invariant Hermitian form, always assuming that $G$ is equal rank. If $G$ is not equal rank, there is really no parallel result, as one can see by investigating the signatures of invariant Hermitian forms on finite-dimensional representations of $S L(2, \mathbb{C})$ and $S L(3, \mathbb{R})$. The difficulty is that there is no element of $G$ whose adjoint action is the Cartan involution $\theta$. In the next section we will address this problem by enlarging $G$ slightly to an extended group ${ }^{\delta} G$ (containing $G$ as a subgroup of index two). The key property of the extended group is that the Cartan involution is inner, and therefore we get an analogue of Theorem 11.2. On the other hand, Clifford theory provides a very close and precise relationship between representation theory for $G$ and for ${ }^{\delta} G$. In particular, information about signatures of invariant Hermitian forms can be passed between the two groups.

\section{Twisting by $\theta$}

We begin as in Theorem 3.4 with a real reductive algebraic

$$
G=G(\mathbb{R}, \sigma) \subset G(\mathbb{C}),
$$

and a maximal compact subgroup

$$
K=G^{\theta} .
$$

Choose a maximal torus (a maximal connected abelian subgroup)

$$
T_{f} \subset K,
$$


and define

$$
H_{f}=Z_{G}\left(T_{f}\right),
$$

a fundamental maximal torus in $G$. It turns out that $H_{f}$ is the group of real points of

$$
H_{f}(\mathbb{C})=Z_{G(\mathbb{C})}\left(T_{f}\right),
$$

and that this group is a complex maximal torus. Clearly $H_{f}$ is uniquely defined up to conjugation by $K$. Recall from Definition 4.1 the dual lattices

$$
X^{*}={ }_{\text {def }} \operatorname{Hom}_{\mathrm{alg}}\left(H_{f}(\mathbb{C}), \mathbb{C}^{\times}\right), \quad X_{*}={ }_{\text {def }} \operatorname{Hom}_{\mathrm{alg}}\left(\mathbb{C}^{\times}, H_{f}(\mathbb{C})\right) ;
$$

the automorphism $\theta$ acts on these lattices (as a lattice automorphism of order two). Recall from Definition 5.2 the finite subsets (in bijection) of roots and coroots

$$
R=R\left(G, H_{f}\right) \subset X^{*}, \quad R^{\vee}=R^{\vee}\left(G, H_{f}\right) \subset X_{*} ;
$$

these subsets are preserved by $\theta$. Because every root has a nontrivial restriction to $T_{f}$ (this is equivalent to the assertion already used that $Z_{G}\left(T_{f}\right)$ is abelian) we can choose a system $R^{+}$of positive roots so that

$$
\theta\left(R^{+}\right)=R^{+}, \quad \theta(\Pi)=\Pi
$$

here $\Pi$ is the set of simple roots for $R^{+}$(Definition 5.2). We define

$$
t_{\mathrm{RD}}=\text { action of } \theta \text { on }\left(X^{*}, \Pi, X_{*}, \Pi^{\vee}\right) \text {; }
$$

the quadruple on the right is the based root datum of $G(\mathbb{C})([37], 16.2 .1)$.

We need to lift the automorphism $t_{\mathrm{RD}}$ to $G(\mathbb{C})$. Of course the Cartan \{eq: basedrootdatumaut $\}$ involution $\theta$ is such a lift; but it is convenient to make a simpler and more canonical choice. For every simple root $\alpha \in \Pi$, we fix a root vector

$$
X_{\alpha} \in \mathfrak{g}_{\alpha} \quad(\alpha \in \Pi) .
$$

This choice of simple root vectors is called a pinning for the based root datum of (12.1i). We can (and do) make these choices in such a way that

$$
\theta\left(X_{\beta}\right)=X_{\theta \beta} \quad(\beta \neq \theta \beta \in \Pi) .
$$

Since $\theta^{2}=1$, we must have

$$
\theta\left(X_{\gamma}\right)=\epsilon(\gamma) X_{\gamma}= \pm X_{\gamma} \quad(\gamma=\theta \gamma \in \Pi)
$$


These choices then define root vectors $X_{-\alpha}$ by the requirement

$$
\left[X_{\alpha}, X_{-\alpha}\right]=H_{\alpha}=d \alpha^{\vee}(1)=d \phi_{\alpha}\left(\begin{array}{cc}
1 & 0 \\
0 & -1
\end{array}\right) \quad(\alpha \in \Pi)
$$

(notation as in Definition 5.2). Finally, these choices of root vectors may be made compatibly with the compact real form $\sigma_{c}$ of Theorem 3.4:

$$
\sigma_{c}\left(X_{\alpha}\right)=-X_{-\alpha}=(\alpha \in \Pi) .
$$

It is a standard fact (see for example [37], Theorem 9.6.2) that any automorphism (like $t_{\mathrm{RD}}$ ) of the based root datum has a unique lift to an automorphism of algebraic groups

$$
\delta_{f}: G(\mathbb{C}) \rightarrow G(\mathbb{C})
$$

preserving $H_{f}$, acting by the automorphism $t_{\mathrm{RD}}$ on $X^{*}\left(H_{f}(\mathbb{C})\right)$, and satisfying

$$
\delta_{f}\left(X_{\alpha}\right)=X_{t_{\mathrm{RD}}(\alpha)}=(\alpha \in \Pi) .
$$

Because of the uniqueness of $\delta_{f}$ and the explicit formulas given above for $\theta$ and $\sigma_{c}$, we see that

$$
\delta_{f}^{2}=1, \quad \delta_{f} \theta=\theta \delta_{f}, \quad \delta_{f} \sigma_{c}=\sigma_{c} \delta_{f} .
$$

\{eq: sigmathetacomm $\}$

We emphasize that the based root datum automorphism $t_{\mathrm{RD}}$ is determined canonically by $\theta$, and in fact just by the inner class of $\theta$ (that is, by the $\operatorname{Ad}(G(\mathbb{C}))$ coset of $\theta$ in $\operatorname{Aut}(G))$. The lift $\delta_{f}$ to $G(\mathbb{C})$ is determined by $t_{\mathrm{RD}}$ and the choice of pinning.

Definition 12.3. With notation as in (12.2), the extended group for $G(\mathbb{C})$ is the semidirect product

$$
{ }^{\delta} G(\mathbb{C})=G(\mathbb{C}) \rtimes\left\{1, \delta_{f}\right\} .
$$

According to (12.2), the automorphism $\delta_{f}$ preserves the subgroups $G$, $K$, and $K(\mathbb{C})$. We can therefore form all of the corresponding extended groups, for example

$$
{ }^{\delta} G=G \rtimes\left\{1, \delta_{f}\right\} .
$$

Because of $(12.2 \mathrm{~h})$, the real forms $\sigma_{0}$ and $\sigma_{c}$ both extend to real forms of ${ }^{\delta} G(\mathbb{C})$ acting trivially on $\delta_{f}$ :

$$
{ }^{\delta} G\left(\mathbb{R}, \sigma_{0}\right)={ }^{\delta} G, \quad{ }^{\delta} K\left(\mathbb{R}, \sigma_{0}\right)={ }^{\delta} K
$$


and so on.

A strong involution for the real form $G$ is an element

$$
x=x_{0} \delta_{f} \in G(\mathbb{C}) \delta_{f}={ }^{\delta} G(\mathbb{C}) \backslash G(\mathbb{C})
$$

with the property that

$$
\left.\operatorname{Ad}(x)\right|_{G(\mathbb{C})}=\theta
$$

In particular, this implies that

$$
x \in{ }^{\delta} K(\mathbb{C}), \quad x^{2}=z \in Z(G(\mathbb{C})) .
$$

By construction of $\delta_{f}$, strong involutions for $G$ must exist. In fact, since $\delta_{f}$ and $\theta$ are automorphisms of $G(\mathbb{C})$ that agree on the $\operatorname{Cartan} H_{f}(\mathbb{C})$, we must have

$$
x_{0} \in H_{f}(\mathbb{C}), \quad x_{0}^{2}=x^{2}=z \in Z(G(\mathbb{C})) .
$$

More precisely, the construction of $\delta_{f}$ in (12.2) shows that $x_{0}$ must satisfy

$$
\begin{aligned}
\beta\left(x_{0}\right)=1 & (\beta \neq \theta(\beta) \in \Pi) \\
\gamma\left(x_{0}\right)=\epsilon(\gamma)= \pm 1 & (\gamma=\theta(\gamma) \in \Pi) .
\end{aligned}
$$

Conversely, any solution $x_{0}$ of these equations determines a strong involution for $G$. Evidently we can find such a solution with the additional property

$$
x_{0} \in\left(H_{f}^{\theta}\right)_{0} \text { has finite order. }
$$

The finite order hypothesis implies that $\sigma_{c}\left(x_{0}\right)=x_{0}$ (since $\sigma_{c}$ defines the unique compact real form of $H_{f}(\mathbb{C})$ ); so $x_{0}$ is also fixed by $\sigma_{0}=\theta \circ \sigma_{c}$, and therefore

$$
x_{0} \in K \subset G \text {. }
$$

Definition 12.5. Suppose $\Gamma=(\Lambda, \nu)$ is a Langlands parameter, written $\{$ def : thetaparam as in Proposition 6.6). The $\theta$-twist of $\Gamma$ is

$$
\Gamma^{\theta}=(\Lambda, \theta \nu) .
$$

In the case of real infinitesimal character (that is, if $\nu$ is real-valued) this is the same as the Hermitian dual (Definition 10.3):

$$
\Gamma^{\theta}=\Gamma^{h, \sigma_{0}} \quad(\nu=\bar{\nu}) .
$$


The next result uses Clifford theory to lift the Langlands classification of irreducible representations of $G$ (Proposition 6.6) to the extended group ${ }^{\delta} G$. Just as for $G$, it says that each irreducible module $J^{\prime}$ is the unique irreducible quotient of a "standard module" $I^{\prime}$.

Proposition 12.6. In the setting of Definition 12.3, fix a strong involution \{prop:Gdeltareps\} $x=x_{0} \delta$ for $G$ as in (12.4) (so that $x_{0} \in K$, and $x^{2}=z \in Z(G) \cap K$ ). Fix an irreducible $(\mathfrak{g}, K)$-module $J$, corresponding to a Langlands parameter $\Gamma=(\Lambda, \nu)$ (Proposition 6.6); write $I_{\text {quo }}$ for the standard module of which $J$ is the unique irreducible quotient.

1. The twists $J^{\delta_{f}}$ and $J^{\theta}=J^{x}$ (Definition 8.11) are isomorphic (by the linear map by which $x_{0} \in K$ acts on $\left.J\right)$. Either twist therefore defines an action of $\left\{1, \delta_{f}\right\}$ on the set $\widehat{G}$ (Definition 2.14).

2. The twist $J^{\theta}$ corresponds to the Langlands parameter $\Gamma^{\theta}$.

3. If $J^{\theta} \simeq J$, then $J$ admits exactly two extensions $J_{ \pm}$to an irreducible module for ${ }^{\delta} G$, differing by the sign character of ${ }^{\delta} G / G \simeq\left\{1, \delta_{f}\right\}$. In this case $J$ or $J_{ \pm}$is type one (Definition 8.14).

4. If $J^{\theta} \simeq J$, then also $I_{\text {quo }}^{\theta} \simeq I_{\text {quo }}$, and this module has exactly two extensions $I_{\mathrm{quo}, \pm}$ to a module for ${ }^{\delta} G$. Each extension $I_{\mathrm{quo}, \pm}$ has a unique irreducible quotient $J_{ \pm}$, one of the two extensions of $J$.

5. If $J^{\theta} \not J$, then

$$
J_{\text {ind }}=\operatorname{Ind}_{(\mathfrak{g}, K)}^{(\mathfrak{g}, \delta)}(J)=J \oplus J^{\theta}
$$

is the unique irreducible $\left(\mathfrak{g},{ }^{\delta} K\right)$-module containing $J$. In this case $J$ and $J^{\theta}$ and $J_{\text {ind }}$ are type two (Definition 8.14).

6. If $J^{\theta} \not J$, then also $I_{\text {quo }}^{\theta} \nsucceq I_{\text {quo }}$, and

$$
I_{\text {quo }, i n d}=\operatorname{Ind}_{(\mathfrak{g}, K)}^{(\mathfrak{g}, \delta)}\left(I_{\text {quo }}\right)=I_{\text {quo }} \oplus I_{\text {quo }}^{\theta}
$$

has $J_{\text {ind }}=J_{\text {ind }}^{\theta}$ as its unique irreducible quotient.

This is essentially a specialization of Proposition 8.13 (Clifford theory) to ${ }^{\delta} G$. The labels \pm in (4) and (5) do not have any particular meaning; it may not be the case that one of the two extensions is preferred.

The modules $I_{\mathrm{quo}, \pm}$ and $I_{\mathrm{quo}, \text { ind }}$ are called standard modules; of course we can in exactly the same way define $I_{\mathrm{sub}, \pm}$ and $I_{\text {sub,ind }}$. 
The only part of the Langlands classification that behaves a little differently for ${ }^{\delta} G$ is that in the case $J^{\theta} \not \mathcal{}$, the lowest ${ }^{\delta} K$-types of $J_{\text {ind }}$ and $I_{\text {ind }}$ have multiplicity two (rather than one as in the classical case).

Now Proposition 10.7 lifts easily to the extended group. For brevity we write only the case of real infinitesimal character.

Proposition 12.7. Suppose $J^{\prime}$ is an irreducible $\left(\mathfrak{g},{ }^{\delta} K\right)$-module of real infinitesimal character, and $I_{\text {quo }}^{\prime}$ is the corresponding standard module.

1. The module $J^{\prime}$ admits a nondegenerate c-invariant Hermitian form $\langle,\rangle^{c}$ that is unique up to a real scalar multiple. It may be chosen to be positive definite on the lowest ${ }^{\delta} K$-types of $J^{\prime}$.

2. The module $I_{\text {quo }}^{\prime}$ admits a nonzero c-invariant form that is unique up to a real scalar. This form has radical equal to the maximal proper submodule $I_{1}^{\prime}$ of $I_{\text {quo }}^{\prime}$, and factors to a nondegenerate form on $J^{\prime}$.

3. If $J^{\prime}$ restricts to an irreducible $J$ for $G$ (so that also $I_{\text {quo }}^{\prime}=I_{\text {quo }}$ ) then the c-invariant forms for ${ }^{\delta} G$ are the forms for $G$.

4. If $J^{\prime}=\operatorname{Ind}(J)=J+J^{\theta}$, then the c-invariant form on $J^{\prime}$ is equal to the orthogonal sum of the form for $J$ on both $J$ and $J^{\theta}$ (using the identification of the vector spaces for $J$ and $J^{\theta}$ (Definition 8.11). A similar statement holds for $I^{\prime}$.

This is immediate from Proposition 10.7. The result allows us to pass information about signatures of $c$-invariant forms back and forth between $G$ and ${ }^{\delta} G$ with no difficulty.

There is one possible confusing point. The outer automorphism $\delta_{f}$ need not act trivially on $\mathfrak{Z}(\mathfrak{g})$, so the irreducible $J^{\prime}$ may not be annihilated by a maximal ideal $\mathfrak{m}$ but only by a product $\mathfrak{m m}^{\delta_{f}}$ of two maximal ideals. But the property that a maximal ideal correspond to real infinitesimal character is preserved by $\delta_{f}$, so in this case what we mean by "real infinitesimal character" for $J^{\prime}$ is that either of these two maximal ideals has real infinitesimal character.

Definition 12.8. Suppose that $G$ is a real reductive algebraic group with extended group ${ }^{\delta} G$ (Definition 12.3). Fix a strong involution $x \in$ ${ }^{\delta} K \backslash K$ as in (12.4), so that

$$
\theta=\operatorname{Ad}(x), \quad x^{2}=z \in Z\left({ }^{\delta} G\right) \cap K
$$


For every $\zeta \in \mathbb{C}^{\times}$, define

$$
\widehat{{ }^{\circ} G_{\zeta}}=\left\{V^{\prime} \in \widehat{{ }^{\circ} G} \mid z \cdot v=\zeta v \quad\left(v \in V^{\prime}\right)\right\},
$$

the set of irreducible $\left(\mathfrak{g},{ }^{\delta} K\right)$-modules in which $z$ acts by $\zeta$. Similarly define $\widehat{K}_{\zeta}$.

Fix a square root $\zeta^{1 / 2}$ of $\zeta$. On every $\mu^{\prime} \in \widehat{\delta}_{{ }^{\prime}}, x$ must act by some square root of $\zeta$; so there is a sign

$$
\epsilon\left(\mu^{\prime}\right) \in\{ \pm 1\}, \quad \mu^{\prime}(x)=\epsilon\left(\mu^{\prime}\right) \zeta^{1 / 2} \quad\left(\mu^{\prime} \in \widehat{\delta} \widehat{K}_{\zeta}\right) .
$$

Of course $\epsilon\left(\mu^{\prime}\right)$ depends on the choice of square root $\zeta^{1 / 2}$.

Theorem 12.9. Suppose $G$ is a real reductive algebraic group with extended $\{$ thm:ctoinv $\}$ group ${ }^{\delta} G$ (Definition 12.3). Fix a strong involution $x$ for $G$, an eigenvalue $\zeta$ for the central element $z$, and a square root $\zeta^{1 / 2}$ of $\zeta$ as in Definition 12.8.

Suppose $J^{\prime} \in \widehat{\delta}_{\zeta}$ is an irreducible of real infinitesimal character. Write $\langle,\rangle_{J^{\prime}}^{c}$ for a c-invariant Hermitian form on $J^{\prime}$ positive on the lowest ${ }^{\delta} K$-types (which exists by Proposition 12.7). Then $J^{\prime}$ admits an invariant Hermitian form $\langle,\rangle_{J^{\prime}}^{0}$ defined by the formula

$$
\langle v, w\rangle_{J^{\prime}}^{0}=\zeta^{-1 / 2}\langle x \cdot v, w\rangle_{J^{\prime}}^{c}=\zeta^{1 / 2}\langle v, x \cdot w\rangle_{J^{\prime}}^{c}
$$

For each $\mu^{\prime} \in \widehat{\delta}^{K_{\zeta}}$, the forms on the $\mu^{\prime}$ multiplicity spaces (Proposition 8.9) are therefore related by

$$
\langle,\rangle_{\left(J^{\prime}\right) \mu^{\mu^{\prime}}}^{0}=\epsilon\left(\mu^{\prime}\right)\langle,\rangle_{\left(J^{\prime}\right) \mu^{\prime}}^{c}
$$

the sign $\epsilon\left(\mu^{\prime}\right)= \pm 1$ is defined in Definition 12.8. Consequently the signature functions are related by

$$
\left(\operatorname{pos}_{J^{\prime}}^{0}\left(\mu^{\prime}\right), \operatorname{neg}_{J^{\prime}}^{0}\left(\mu^{\prime}\right)\right)= \begin{cases}\left(\operatorname{pos}_{J^{\prime}}^{c}\left(\mu^{\prime}\right), \operatorname{neg}_{J^{\prime}}^{c}\left(\mu^{\prime}\right)\right) & \left(\epsilon\left(\mu^{\prime}\right)=+1\right) \\ \left(\operatorname{neg}_{J^{\prime}}^{c}\left(\mu^{\prime}\right), \operatorname{pos}_{J^{\prime}}^{c}\left(\mu^{\prime}\right)\right) & \left(\epsilon\left(\mu^{\prime}\right)=-1\right)\end{cases}
$$

Proof. The formula for the invariant Hermitian form is a special case of Proposition 8.16; by Cartan's original construction of the Cartan involution, the two automorphisms $\delta$ and $\epsilon$ of that proposition are both $\theta$, and we can choose $\lambda=x$. The statements about signatures are immediate. 


\section{Langlands parameters for extended groups}

Proposition 12.6 provides a nice abstract description of the irreducible representations of an extended group. For the unitarity algorithm which is our goal, we will need something more concrete and precise. This is of the same nature as what we have already done with the formulation of the Langlands classification in Theorem 6.1, which replaced the "tempered representation of a Levi subgroup" appearing in [24] with a character of an appropriate cover of a real maximal torus. Proposition 12.6 says that an irreducible representation of $G$ fixed by $\theta$ and corresponding to a Langlands parameter has two extensions to ${ }^{\delta} G$. We are going to index these two extensions by two extensions of the parameter.

Throughout this section we fix an extended group

$$
{ }^{\delta} G=G \rtimes\left\{1, \delta_{f}\right\}
$$

as in Definition 12.3, and a strong involution

$$
x=x_{0} \delta_{f} \in{ }^{\delta} K \backslash K, \quad \operatorname{Ad}(x)=\theta, \quad x^{2}=z \in Z(G) \cap K
$$

as in (12.4). We fix also a $\theta$-stable maximal torus $H \subset G$ (Definition 5.7). Evidently $H$ is normalized by $x$, so we can consider the extended group

$$
{ }^{x} H=\langle H, x\rangle,
$$

and try to extend a Langlands parameter to a character of ${ }^{x} H$. This is almost always impossible when $H$ is not fundamental (see (12.1)). The reason is that what is preserved by $\theta$ is not the parameter but only its conjugacy class under $K$.

The point of this section is to find replacements for ${ }^{x} H$ to which Langlands parameters can usefully extend, and in this way to parametrize representations of ${ }^{\delta} G$. We begin with basic information about the extended Weyl group.

Proposition 13.2. In the setting of Definition 12.3 and (13.1), write $H(\mathbb{C})$ $\{$ prop: extWC for the complexification of the $\theta$-stable real torus $H$. Fix a maximal torus $H(\mathbb{C})$ and a system of positive roots

$$
R_{1}^{+} \subset R(G, H)
$$

with simple roots $\Pi_{1}$. Write

$$
S_{1}=\left\{s_{\alpha} \mid \alpha \in \Pi_{1}\right\} \subset W(G(\mathbb{C}), H(\mathbb{C}))=W
$$


for the corresponding set of simple reflections (Definition 5.2). Define

$$
{ }^{\delta} W={ }_{\operatorname{def}} N_{\delta} G(\mathbb{C})(H(\mathbb{C})) / H(\mathbb{C}) \supset W
$$

the extended Weyl group of $H(\mathbb{C})$ in $G(\mathbb{C})$.

1. There is a unique nonidentity class

$$
t_{1}\left(R_{1}^{+}\right)=t_{1} \in{ }^{\delta} W
$$

with the property that $t_{1} \cdot R_{1}^{+}=R_{1}^{+}$.

2. Conjugation by $t_{1}$ permutes the generators $S_{1}$ of $W$, and provides a semidirect product decomposition

$$
{ }^{\delta} W=W \rtimes\left\{1, t_{1}\right\} .
$$

Write $T_{1}$ for the set of orbits of $\left\{1, t_{1}\right\}$ on $S_{1}$. We identify $T_{1}$ with a collection of elements of order 2 in $W$, as follows. Suppose

$$
t=\left\{s, s^{\prime}\right\} \quad s^{\prime}=t_{1}(s) .
$$

Then we associate the orbit $t$ to an element of $W$ by

$$
\begin{aligned}
& t \leftrightarrow \text { long element of }\left\langle s, s^{\prime}\right\rangle \\
& =\left\{\begin{array}{lll}
s & t=\left\{s=s^{\prime}\right\} & \\
s s^{\prime} & t=\left\{s \neq s^{\prime}\right\}, & s s^{\prime}=s^{\prime} s \\
s s^{\prime} s=s^{\prime} s s^{\prime} & t=\left\{s \neq s^{\prime}\right\}, & s s^{\prime} \neq s^{\prime} s .
\end{array}\right.
\end{aligned}
$$

Each of these elements has order two and is fixed by $t_{1}$.

3. The group $W^{t_{1}}$ of fixed points of the automorphism $t_{1}$ acts as a reflection group (in fact a Weyl group) on the lattice $\left(X^{*}\right)^{t_{1}}$ of $t_{1}$-fixed characters of $H(\mathbb{C})$. The set $T_{1}$ described above is a set of Coxeter generators.

4. Recall from (12.1) the fundamental maximal torus $H_{f}$ and positive root system $R_{f}^{+}$used to construct the extended group. There is an element $g \in G(\mathbb{C})$ with the property that

$$
\operatorname{Ad}(g)\left(H_{f}(\mathbb{C})\right)=H(\mathbb{C}), \quad \operatorname{Ad}(g)\left(R_{f}^{+}\right)=R_{1}^{+} .
$$

The coset $g H_{f}(\mathbb{C})=H(\mathbb{C}) g$ is unique. 
5. Conjugation by $g$ defines a canonical isomorphism

$$
{ }^{\delta} W\left(G(\mathbb{C}), H_{f}(\mathbb{C})\right) \stackrel{\simeq}{\longrightarrow}{ }^{\delta} W(G(\mathbb{C}), H(\mathbb{C})),
$$

carrying $t_{f}$ to $t_{1}$ and $S_{f}$ (the simple reflections for $W\left(G(\mathbb{C}), H_{f}(\mathbb{C})\right)$ ) to $S_{1}$.

6. Conjugation by $g$ carries the lift $\delta_{f} \in{ }^{\delta} G$ of $t_{f}$ to a lift

$$
\delta_{1} \in N_{\delta_{G(\mathbb{C})}}(H(\mathbb{C}))
$$

of $t_{1}$. The $H(\mathbb{C})$ conjugacy class of $\delta_{1}$-i.e., the coset $\delta_{1}\left[H(\mathbb{C})^{-t_{1}}\right]_{0}-i$ s unique. We call these the distinguished lifts of $t_{1}$.

This is an immediate consequence of the corresponding facts about the Weyl group (see for example [37], Corollary 6.4.12 and Proposition 8.24).

Definition 13.3. In the setting of Proposition 13.2, a twisted involution

in $W$ is an element $w_{1} \in W$ with the property that

$$
w_{1} t_{1}\left(w_{1}\right)=1 \text {. }
$$

The twisted conjugate of $w_{1}$ by $y \in W$ is

$$
w_{1}^{\prime}=y w_{1}\left[t_{1}(y)^{-1}\right] \text {. }
$$

Clearly $w_{1}$ is a twisted involution if and only if $w_{1} t_{1}$ is an involution in the nonidentity coset $W t_{1} \subset{ }^{\delta} W$. In this correspondence, $w_{1}^{\prime}$ is a twisted conjugate of $w_{1}$ if and only if $w_{1}^{\prime} t_{1}$ is a $W$-conjugate of $w_{1} t_{1}$.

Lemma 13.4. Suppose we are in the setting of Proposition 13.2 and Definition 13.3 .

1. Each twisted conjugacy class of twisted involutions has a representative

$$
w_{1}=s_{\beta_{1}} s_{\beta_{2}} \cdots s_{\beta_{m}}
$$

with $\left\{\beta_{i}\right\}$ an orthogonal collection of $t_{1}$-fixed roots.

2. The number $m$ is an invariant of the twisted conjugacy class of $w_{1}$. We have

$$
m+\operatorname{dim}\left(-1 \text { eigenspace of } t_{1}\right)=\operatorname{dim}\left(-1 \text { eigenspace of } w_{1} t_{1}\right) .
$$

3. The following conditions on an involution $t_{2} \in W t_{1}$ are equivalent: 
i) $t_{2}$ is conjugate by $W$ to $t_{1}$;

ii) $t_{2}$ preserves some system of positive roots $R_{2}^{+}$;

iii) $\operatorname{dim}\left(-1\right.$ eigenspace of $\left.t_{2}\right)=\operatorname{dim}\left(-1\right.$ eigenspace of $\left.t_{1}\right)$

Proof. Write

$$
\mathfrak{h}_{\mathbb{Q}}^{*}=X^{*} \otimes_{\mathbb{Z}} \mathbb{Q}
$$

for the rational part of the dual of the Cartan. Decompose this space as

$$
\mathfrak{h}_{\mathbb{Q}}^{*}=V_{1}\left(w_{1} t_{1}\right) \oplus V_{-1}\left(w_{1} t_{1}\right)
$$

according to the eigenspaces of $w_{1} t_{1}$. We prove (1) by induction on the dimension $d$ of $V_{-1}\left(w_{1} t_{1}\right)$. There are no elements for which $d=-1$, so in that case the assertion in (1) is empty.

Suppose therefore that $d \geq 0$, and that the result is known for $d-1$. There are two cases. Suppose first that there is a root $\gamma_{1} \in V_{-1}\left(w_{1} t_{1}\right)$. Put $w_{2}=s_{\gamma_{1}} w_{2}$, so that

$$
V_{1}\left(w_{2} t_{1}\right)=V_{1}\left(w_{1} t_{1}\right) \oplus \mathbb{Q} \beta, V_{-1}\left(w_{2} t_{1}\right)=\operatorname{ker}\left(\beta^{\vee}\right) \cap V_{-1}\left(w_{1} t_{1}\right) .
$$

Clearly $w_{2} t_{1}$ is an involution with -1 eigenspace of dimension $d-1$, so by induction

$$
w_{2} t_{1}=y\left[s_{\beta_{2}} s_{\beta_{3}} \cdots s_{\beta_{m}}\right] t_{1} y^{-1} .
$$

If we define $\beta_{1}=y^{-1} \cdot \gamma_{1}$, then it is easy to check that (1) holds for $w_{1}$.

Next, suppose that there is no root $\gamma \in V_{-1}\left(w_{1} t_{1}\right)$. We may therefore choose a weight $\xi_{2} \in V_{1}\left(w_{1} t_{1}\right)$ vanishing on no coroots; for the only coroots $\gamma^{\vee}$ on which every element of $V_{1}$ vanishes are those with $\gamma \in V_{-1}$. Now define

$$
R_{2}^{+}=\left\{\alpha \in R \mid \xi_{2}\left(\alpha^{\vee}\right)>0\right\} .
$$

This is a system of positive roots, and

$$
w_{1} t_{1}\left(R_{2}^{+}\right)=R_{2}^{+}
$$

since $w_{1} t_{1}$ fixes $\gamma_{2}$. Therefore $w_{1} t_{1}$ must be the unique element $t_{2} \in W t_{1}$ fixing $R_{2}^{+}$. If $R_{2}^{+}=y R_{1}^{+}$, then evidently

$$
w_{1} t_{1}=t_{2}=y t_{1} y^{-1}
$$

This proves (1) (with $m=0$ ).

The remaining assertions in the lemma are elementary consequences of (1). 
Definition 13.5. In the setting (13.1), the extended real Weyl group of $H$ is

$$
\begin{aligned}
{ }^{\delta} W(G, H) & =N_{\delta_{G}}(H) / H \simeq N_{\delta_{K}}(H) /\left(H \cap{ }^{\delta} K\right) \\
& \subset{ }^{\delta} W(G(\mathbb{C}), H(\mathbb{C}))
\end{aligned}
$$

(notation as in Definition 5.7 and Proposition 13.2). According to (13.1), the extended real Weyl group always has an element

$$
x H={ }_{\operatorname{def}} \theta_{H},
$$

which is characterized by the two properties

$\theta_{H} \notin W(G(\mathbb{C}), H(\mathbb{C})), \quad \theta_{H}$ acts on $H$ by the Cartan involution.

Consequently ${ }^{\delta} W(G, H)$ inherits from ${ }^{\delta} W(G(\mathbb{C}), H(\mathbb{C}))$ the short exact sequence

$$
1 \rightarrow W(G, H) \rightarrow{ }^{\delta} W(G, H) \rightarrow\{1, \delta\} \rightarrow 1 ;
$$

but the splitting of this sequence by $\theta_{H}$ is not one of the nice ones described in Proposition 13.2(2).

An extended maximal torus in ${ }^{\delta} G$ is a subgroup ${ }^{1} H \supset H$, subject to the following conditions.

a) The group ${ }^{1} H$ is not contained in $G$, and $\left[{ }^{1} H: H\right]=2$. Equivalently, we require that ${ }^{1} H$ be generated by $H$ and a single element

$$
t_{1}=w_{1} \theta_{H} \in{ }^{\delta} W(G, H) \backslash W(G, H),
$$

of order two.

b) There is a set $R_{1}^{+} \subset R=R(G, H)$ of positive roots preserved by $t_{1}$.

We do not include a particular choice of $R_{1}^{+}$as part of the definition of ${ }^{1} H$. Typically we will write something like $\delta_{1} \in{ }^{1} H \cap{ }^{\delta} K$ for a representative of $t_{1}$.

The first thing to notice is that the obvious group $\left\langle H, \theta_{H}\right\rangle$ (generated by $H$ and the strong involution $x$ of (12.4)) is not an extended maximal torus unless the set $R_{\mathbb{R}}^{+}$of real roots is empty; that is, unless $H$ is fundamental. The reason we impose the requirement b) above is that representationtheoretic information is typically encoded not just by a Cartan subalgebra, but rather by a Borel subalgebra containing it. (This is the central idea in the theory of highest weights.)

Nevertheless, every maximal torus is contained in an extended maximal torus. 
Example 13.6. Following (9.3), choose $\mathfrak{q}=\mathfrak{l}+\mathfrak{u}$, so that the roots of $H$ in in $\mathfrak{l}$ are precisely the real roots $R_{\mathbb{R}}$ of $H$ in $\mathfrak{g}$. Fix a set of positive real roots $R_{\mathbb{R}}^{+}$, and define

$$
R_{\mathrm{VZ}}^{+}=R_{\mathbb{R}}^{+} \cup\{\text { roots of } H \text { in } \mathfrak{u}\} .
$$

Write $w_{0, \mathbb{R}}$ for the long element of $W\left(R_{\mathbb{R}}\right)$, which carries $R_{\mathbb{R}}^{+}$to $-R_{\mathbb{R}}^{+}$, and $\sigma_{0, \mathbb{R}}$ for a representative of this Weyl group element in $N_{K}(H)$. Because $\mathfrak{u}$ is preserved by $\theta$, we find that

$$
w_{0, \mathbb{R}} \theta_{H}\left(R_{1}^{+}\right)=R_{1}^{+} .
$$

Therefore $H$ and $\delta_{\mathrm{VZ}}=\sigma_{0, \mathbb{R}} \cdot x$ generate an extended maximal torus ${ }^{\mathrm{VZ}} H$, with $t_{\mathrm{VZ}}=w_{0, \mathbb{R}} \theta_{H}$.

Definition 13.7. Suppose ${ }^{1} H$ is an extended maximal torus in ${ }^{\delta} G$. An $\{$ def : extLP $\}$ extended Langlands parameter is a triple $\Gamma_{1}=\left({ }^{1} H, \gamma_{1}, R_{i \mathbb{R}}^{+}\right)$, subject to the following conditions.

a) The group ${ }^{1} H$ is an extended maximal torus for ${ }^{\delta} G$, with distinguished generator $t_{1} \in{ }^{\delta} W(G, H)$ (Definition 13.5).

b) The element $t_{1}$ preserves the positive imaginary roots $R_{i \mathbb{R}}^{+}$from $\Gamma_{1}$.

c) As a consequence of (2), the character $2 \rho_{i \mathbb{R}}$ of $H$ extends naturally to ${ }^{1} H$, and so defines the $\rho_{i \mathbb{R}}$ double cover $\widetilde{{ }^{1} H}$ (Lemma 5.9). We require that $\gamma_{1}$ be a level one irreducible representation of this cover (and therefore of dimension one or two).

d) The restriction of $\gamma_{1}$ to $\widetilde{H}$ is (automatically) a sum of one or two irreducible characters $\gamma$ of the $\rho_{i \mathbb{R}}$ double cover of $H$; and we require that $\Gamma=\left(H, \gamma, R_{i \mathbb{R}}^{+}\right)$be a Langlands parameter for $G$ (Theorem 6.1).

We say that $\Gamma_{1}$ (or $\Gamma$ ) is type one if $\gamma_{1}$ is one-dimensional; equivalently, if the corresponding parameter $\Gamma$ for $G$ is fixed by $t_{1}$. We say that $\Gamma_{1}$ (or $\Gamma$ ) is type two if $\gamma_{1}=\gamma_{\text {ind }}$ is two-dimensional (Definition 8.14). In order to guarantee that a parameter corresponds to an irreducible representation of ${ }^{\delta} G$, we need to require also

e) if $\Gamma_{1}=\Gamma_{\text {ind }}$ is type two, then $\Gamma$ is not equivalent to $\Gamma^{\theta}$.

Just as in Definition 6.3, we can define continued and weak extended parameters; on these we impose only conditions a)-c), and insert "continued" or "weak" in d). We do not require anything like e), just as 
weak Langlands parameters are not required to be final (condition (5) of Theorem 6.1).

Of course one of the things that we want is that type one representations of the extended group (Proposition 12.6) correspond precisely to type one extended parameters. There is a subtlety here. Under hypotheses a)-d), it will be fairly easy to see that a type one extended parameter corresponds to a type one representation, and that a type two representation corresponds to a type two parameter (see the remarks after Lemma 13.10 below). Proposition 13.12 will provide a converse: that any type one representation has a type one parameter. The proof of that proposition shows first of all that in order to find this type one parameter - that is, to find a one-dimensional extension of the character $\gamma$-we must choose the extended torus properly. (There is a type two extension, given by induction, for any extended torus containing $H$.) The point of condition (e) is to require us to make a proper choice of extended torus, so that the type of the representation corresponds to the type of the parameter.

We turn next to the definition of equivalence of extended parameters. The subtlety here arises entirely from the possibility that a single Langlands parameter may extend to two distinct extended tori. Before giving the definition, we recall a little about the stabilizer of a Langlands parameter.

Lemma 13.8. Suppose $\Gamma=(\Lambda, \nu)$ is a Langlands parameter decomposed as \{lemma: stabGamma\} in Definition 6.5, with $\Lambda=\left(\lambda, R_{i \mathbb{R}}^{+}\right)$. Write $\rho_{i \mathbb{R}}$ for half the sum of the roots in $R_{i \mathbb{R}}^{+}$, and $\bar{\lambda}=d \lambda$, so that $\left[\lambda-\rho_{i \mathbb{R}}\right]$ is a character of $T$. Define

$$
R^{0}=\left\{\alpha \in R(\mathfrak{g}, \mathfrak{h}) \mid\left\langle\alpha^{\vee}, \bar{\lambda}\right\rangle=\left\langle\alpha^{\vee}, \nu\right\rangle=\left\langle\alpha^{\vee}, \rho_{i \mathbb{R}}\right\rangle=0\right\},
$$

the set of singular roots for the discrete parameter.

1. The roots $R^{0}$ are the root system for a real quasisplit Levi subgroup $L^{0}$ of $G$.

2. The real Weyl group $W\left(L^{0}, H\right)$ is equal to the centralizer of $\theta_{H}$ in $W\left(\mathfrak{l}^{0}, \mathfrak{h}\right)$.

3. If $\alpha$ is a real root in $R^{0}$, then $s_{\alpha} \cdot \Lambda=\Lambda$, and $\left[\lambda-\rho_{i \mathbb{R}}\right]\left(m_{\alpha}\right)=1$ (notation as in Definition 5.7).

4. The stabilizer of $\Gamma$ in $W(G, H)$ is equal to $W\left(L^{0}, H\right)$.

5. The character $\left[\lambda-\rho_{i \mathbb{R}}\right]$ of $T$ is trivial on the intersection of $T$ with the identity component of the derived group $\left[L^{0}, L^{0}\right]$. 
Definition 13.9. Two extended parameters of type two are said to be equivalent if the underlying Langlands parameters are equivalent.

In the type one case, we need to be concerned about the possibility that the extended torus to which $\Gamma$ extends is not unique. Suppose therefore that $\Gamma$ is a Langlands parameter on a maximal torus $H$, and that

$$
{ }^{1} H=\left\langle H, t_{1}\right\rangle, \quad{ }^{2} H=\left\langle H, t_{2}\right\rangle
$$

are two extended tori to which $\Gamma$ extends; that is, that

$$
\Gamma^{t_{1}}=\Gamma^{t_{2}}=\Gamma \text {. }
$$

Therefore $t_{2}=w t_{1}$, with $w \in W(G, H)^{\Gamma}=W\left(L^{0}, H\right)$ (Lemma 13.8). Choose a representative $\delta_{1}$ for $t_{1}$, and a representative $\sigma$ for $w$ belonging to the identity component of the derived group of $L^{0}$. Then

$$
\delta_{2}=\sigma \delta_{1}
$$

is a representative for $t_{2}$.

Suppose $\Gamma_{1}$ and $\Gamma_{2}$ are extensions of $\Gamma$ to ${ }^{1} H$ and ${ }^{2} H$ respectively. We say that $\Gamma_{1}$ is equivalent to $\Gamma_{2}$ if

$$
\left[\lambda_{1}-\rho_{i \mathbb{R}}\right]\left(\delta_{1}\right)=\left[\lambda_{2}-\rho_{i \mathbb{R}}\right]\left(\delta_{2}\right) .
$$

According to Lemma 13.8, this condition is independent of the choice of representative $\delta_{1}$ above. More generally, we say that a type one extended parameter $\Gamma_{1}$ is equivalent to an extended parameter $\Gamma_{3}$ if $\Gamma_{3}$ is conjugate by $K$ to some $\Gamma_{2}$ as above.

We are going to show (Proposition 13.12 below) that the parameter for a type one irreducible representation in fact extends to an appropriate extended torus. The next lemma shows what we need to do.

Lemma 13.10. Suppose $\Gamma=\left(H, \gamma, R_{i \mathbb{R}}^{+}\right)$is a Langlands parameter (Theorem 6.1). Write $H=T A$ and $\Gamma=(\Lambda, \nu)$, with $\Lambda=\left(\lambda, R_{i \mathbb{R}}^{+}\right)$as in Definition 6.5; put $\bar{\lambda}=d \lambda \in \mathfrak{t}^{*}$, so that

$$
(\bar{\lambda}, \nu) \in \mathfrak{t}^{*}+\mathfrak{a}^{*}=\mathfrak{h}^{*}
$$

represents the infinitesimal character of $J(\Gamma)$. Then $\Gamma$ extends to the extended torus ${ }^{1} H=\left\langle H, t_{1}=w_{1} \theta_{H}\right\rangle$ if and only if

1. $w_{1} \lambda=\lambda$; 
2. $w_{1}\left(R_{i \mathbb{R}}^{+}\right)=R_{i \mathbb{R}}^{+}$; and

3. $w_{1} \nu=-\nu$.

These three conditions are equivalent in turn to

$$
w_{1} \cdot \Gamma=\Gamma^{\theta_{H}}:
$$

This is clear from the definitions (since the real Weyl group element $w_{1}$ must commute with the action of $\theta_{H}$ on $H$ ). In particular, the lemma implies that a Langlands parameter $\Gamma$ on $H$ can extend to some extended torus for $H$ only if $\Gamma^{\theta_{H}}$ is conjugate to $\Gamma$ by the real Weyl group; that is (Proposition 12.6) only if $J(\Gamma)$ is type one.

The converse requires a bit more work, on which we now embark. Assume that $J(\Gamma)$ is type one, so that

$$
\Gamma^{\theta_{H}}=w \cdot \Gamma \quad(\text { some } w \in W(G, H)) .
$$

Write $H=T A$ and

$$
\Gamma=(\Lambda, \nu), \quad \Lambda=\left(\lambda, R_{i \mathbb{R}}^{+}\right)
$$

as in Definition 6.5; put $\bar{\lambda}=d \lambda \in \mathfrak{t}^{*}$, so that

$$
(\bar{\lambda}, \nu) \in \mathfrak{t}^{*}+\mathfrak{a}^{*}=\mathfrak{h}^{*}
$$

represents the infinitesimal character of $J(\Gamma)$.

We want to prove that $\Gamma$ extends to some extended torus ${ }^{1} H$, and in fact to understand all possible extended tori to which $\Gamma$ extends. The easiest way to construct an extended torus $\left\langle H, t_{1}\right\rangle$ is to construct a system of positive roots preserved by $t_{1}$; by Proposition 13.2(1), such a system determines $t_{1}$. Since we want also that $t_{1}$ should carry $\Gamma$ to $\Gamma$, it is reasonable to use $\Gamma$ to construct positive roots. To simplify the discussion, and because (in light of Theorem 7.1) it is our primary interest, we consider $\Gamma$ of real infinitesimal character. This means in particular that

$$
\left\langle\alpha^{\vee}, \bar{\lambda}\right\rangle \in \mathbb{R}, \quad\left\langle\alpha^{\vee}, \nu\right\rangle \in \mathbb{R} \quad(\alpha \in R(\mathfrak{g}, \mathfrak{h})) .
$$

(The first of these conditions is automatically true for any $\Gamma$.) If now $(\bar{\lambda}, \nu)$ is regular, then we could define a positive root system

$$
R^{+}(\Gamma)=\left\{\alpha \in R(\mathfrak{g}, \mathfrak{h}) \mid\left\langle\alpha^{\vee},(\bar{\lambda}, \nu)\right\rangle>0\right\}
$$


The only candidate for an extended torus to which $\Gamma$ extends in this case is the one generated by the unique $t_{1} \in W \theta_{H}$ preserving $R^{+}(\Gamma)$. There are two difficulties with this approach in general. One is that $(\bar{\lambda}, \nu)$ need not be regular. The second is that it is not so easy to see whether the element $t_{1}$ defined in this way belongs to the extended real Weyl group $W(G, H) \theta_{H}$.

We therefore take a slightly different approach. Begin with what is (more or less obviously) a "triangular" decomposition of the root system, into the roots of the nil radical of a parabolic subalgebra; the roots of the Levi factor; and the roots of the opposite nil radical:

$$
\begin{aligned}
R^{+}(\bar{\lambda})= & \left\{\alpha \in R(\mathfrak{g}, \mathfrak{h}) \mid\left\langle\alpha^{\vee}, \bar{\lambda}\right\rangle>0\right\} \\
& \cup\left\{\alpha \in R(\mathfrak{g}, \mathfrak{h}) \mid\left\langle\alpha^{\vee}, \bar{\lambda}\right\rangle=0,\left\langle\alpha^{\vee}, \rho_{i \mathbb{R}}\right\rangle>0\right\} \\
R_{0}(\bar{\lambda})= & \left\{\alpha \in R(\mathfrak{g}, \mathfrak{h}) \mid\left\langle\alpha^{\vee}, \bar{\lambda}\right\rangle=0,\left\langle\alpha^{\vee}, \rho_{i \mathbb{R}}\right\rangle=0\right\} \\
R^{-}(\bar{\lambda})= & -R^{+}(\bar{\lambda}) ;
\end{aligned}
$$

here $\rho_{i \mathbb{R}}$ is half the sum of the roots in $R_{i \mathbb{R}}^{+}$. The parabolic subalgebra is

$$
\begin{aligned}
\mathfrak{p}(\bar{\lambda}) & =\mathfrak{l}(\bar{\lambda})+\mathfrak{n}(\bar{\lambda}), \\
R(\mathfrak{n}(\bar{\lambda}), \mathfrak{h}) & =R^{+}(\bar{\lambda}), \quad R(\mathfrak{l}(\bar{\lambda}), \mathfrak{h})=R^{0}(\bar{\lambda}) .
\end{aligned}
$$

$\{$ eq:plambda $\}$

To make a parallel construction for $\nu$, we must fix a system of positive real roots making $\nu$ weakly dominant:

$$
R_{\mathbb{R}}^{+} \supset\left\{\alpha \in R_{\mathbb{R}} \mid\left\langle\alpha^{\vee}, \nu\right\rangle>0\right\} .
$$

It follows from Lemma 13.8 that such a choice of $R_{\mathbb{R}}^{+}$is unique up to the action of $W(G, H)^{\Gamma}$. With this choice in hand, we can define

$$
\begin{aligned}
R^{+}(\nu)= & \left\{\alpha \in R(\mathfrak{g}, \mathfrak{h}) \mid\left\langle\alpha^{\vee}, \nu\right\rangle>0\right\} \\
& \cup\left\{\alpha \in R(\mathfrak{g}, \mathfrak{h}) \mid\left\langle\alpha^{\vee}, \nu\right\rangle=0,\left\langle\alpha^{\vee}, \rho_{\mathbb{R}}\right\rangle>0\right\} \\
R^{0}(\nu)= & \left\{\alpha \in R(\mathfrak{g}, \mathfrak{h}) \mid\left\langle\alpha^{\vee}, \nu\right\rangle=0,\left\langle\alpha^{\vee}, \rho_{\mathbb{R}}\right\rangle=0\right\} \\
R^{-}(\nu)= & -R^{+}(\nu) ;
\end{aligned}
$$

here $\rho_{\mathbb{R}}$ is half the sum of the roots in $R_{\mathbb{R}}^{+}$. The corresponding parabolic subalgebra is written

$$
\begin{aligned}
\mathfrak{p}(\nu) & =\mathfrak{l}(\nu)+\mathfrak{n}(\nu), \\
R(\mathfrak{n}(\nu), \mathfrak{h}) & =R^{+}(\nu), \quad R(\mathfrak{l}(\nu), \mathfrak{h})=R^{0}(\nu) .
\end{aligned}
$$


We now combine these two constructions of parabolic subalgebras, defining

$$
\begin{aligned}
R^{+}(\lambda, \nu) & =R^{+}(\lambda) \cup\left(R^{0}(\lambda) \cap R^{+}(\nu)\right) \\
R^{0}(\lambda, \nu) & =R^{0}(\lambda) \cap R^{0}(\nu) \\
R^{-}(\lambda, \nu) & =-R^{+}(\lambda, \nu) ;
\end{aligned}
$$

The corresponding parabolic subalgebra is written

$$
\begin{aligned}
\mathfrak{p}(\bar{\lambda}, \nu) & =\mathfrak{l}(\bar{\lambda}, \nu)+\mathfrak{n}(\bar{\lambda}, \nu), \\
R(\mathfrak{n}(\bar{\lambda}, \nu), \mathfrak{h}) & =R^{+}(\bar{\lambda}, \nu), \quad R(\mathfrak{l}(\bar{\lambda}, \nu), \mathfrak{h})=R^{0}(\bar{\lambda}, \nu) .
\end{aligned}
$$

$\{$ eq: plambdanu $\}$

We could equally well have reversed the roles of $\bar{\lambda}$ and $\nu$, obtaining a different parabolic subalgebra $\mathfrak{p}(\nu, \bar{\lambda})$. The Levi subalgebra $\mathfrak{r}(\bar{\lambda}, \nu)$, corresponding to the coroots vanishing on $\bar{\lambda}, \nu, \rho_{i \mathbb{R}}$, and $\rho_{\mathbb{R}}$, would be the same. We will see that these two parabolic subalgebras correspond to two constructions of the standard representation for the extended group.

Proposition 13.12. Suppose $\Gamma=\left(H, \gamma, R_{i \mathbb{R}}^{+}\right)$is a Langlands parameter $\{$ prop: extLP $\}$ (Theorem 6.1). Assume that the conjugacy class of $\Gamma$ is fixed by twisting by $\theta$ (equivalently, by $x \in{ }^{\delta} G$ ); equivalently, that the corresponding irreducible representation $J(\Gamma)$ extends to ${ }^{\delta} G$. Write $H=T A$ and $\Gamma=(\Lambda, \nu)$ as in Definition 6.5; put $\bar{\lambda}=d \Lambda \in \mathfrak{t}^{*}$, so that

$$
(\bar{\lambda}, \nu) \in \mathfrak{t}^{*}+\mathfrak{a}^{*}=\mathfrak{h}^{*}
$$

represents the infinitesimal character of $J(\Gamma)$. Fix a system of positive real roots $R_{\mathbb{R}}^{+}$making $\nu$ weakly dominant.

1. The Levi subgroups $L(\nu)$ and $L(\bar{\lambda})$ corresponding to $R^{0}(\nu)$ and $R^{0}(\bar{\lambda})$ are $\theta$-stable and defined over $\mathbb{R}$.

2. The parabolic subalgebra $\mathfrak{p}(\nu)$ is defined over $\mathbb{R}$, and so corresponds to a real parabolic subgroup $P(\nu)=L(\nu) N(\nu)$ of $G$.

3. The parabolic subalgebra $\mathfrak{p}(\bar{\lambda})$ is $\theta$-stable.

4. The group $L(\nu)$ has no real roots for $H$, so $H$ is a fundamental Cartan in $L(\nu)$.

5. The group $L(\bar{\lambda})$ has no imaginary roots for $H$, so $H$ is a maximally split torus in the quasisplit group $L(\bar{\lambda})$.

6. The Levi subgroup $L(\bar{\lambda}, \nu)=L(\bar{\lambda}) \cap L(\nu)$ is real and $\theta$-stable. 
7. There are neither real nor imaginary roots of $H$ in $L(\bar{\lambda}, \nu)$, so $L(\bar{\lambda}, \nu)$ is locally isomorphic to a complex reductive group.

8. The real Weyl group $W(\bar{\lambda}, \nu)$ for $H$ in $L(\bar{\lambda}, \nu)$ is contained in the stabilizer of $\Gamma$.

Choose a real Borel subgroup $B=H N \subset L(\bar{\lambda})$ contained in $P(\nu) \cap L(\bar{\lambda})$, and write

$$
w_{0}(\bar{\lambda})=\text { long element of } W(L(\bar{\lambda}), H) .
$$

Because $L(\lambda, \nu)$ is locally isomorphic to a complex reductive group, we can choose a sum of simple subsystems

$$
R^{L}(\bar{\lambda}, \nu) \subset R(\bar{\lambda}, \nu)
$$

in such a way that

$$
R(\bar{\lambda}, \nu)=R^{L}(\bar{\lambda}, \nu) \amalg \theta R^{L}(\bar{\lambda}, \nu), \quad R^{R}(\bar{\lambda}, \nu)={ }_{\operatorname{def}} \theta R^{L}(\bar{\lambda}, \nu) .
$$

Write

$$
R^{x,+}(\bar{\lambda}, \nu)=\text { roots in } N \quad(x=L, R) .
$$

Because the roots in $N$ are permuted by $-\theta$, we have

$$
\theta R^{L,+}(\bar{\lambda}, \nu)=-R^{R,+}(\bar{\lambda}, \nu) \quad \theta R^{R,+}(\bar{\lambda}, \nu)=-R^{L,+}(\bar{\lambda}, \nu) .
$$

We consider now two different positive root systems for $\mathfrak{h}$ in $\mathfrak{g}$ :

$$
\begin{gathered}
R^{+, 1}=R^{+}(\bar{\lambda}) \cup\left[R^{0}(\bar{\lambda}) \cap R^{+}(\nu)\right] \cup R^{L,+}(\bar{\lambda}, \nu) \cup-R^{R,+}(\bar{\lambda}, \nu), \\
R^{+, 2}=R^{+}(\bar{\lambda}) \cup\left[R^{0}(\bar{\lambda}) \cap R^{+}(\nu)\right] \cup R^{L,+}(\bar{\lambda}, \nu) \cup R^{R,+}(\bar{\lambda}, \nu),
\end{gathered}
$$

9. The element $w_{0}(\bar{\lambda})$ fixes $\Gamma$.

10. The stabilizer of $\left(\Gamma, R_{\mathbb{R}}^{+}\right)$in the coset $W(G, H) \theta_{H}$ is $W(\bar{\lambda}, \nu) w_{0}(\bar{\lambda}) \theta_{H}$.

11. The element $t_{2}={ }_{\text {def }} w_{0}(\bar{\lambda}) \theta_{H}$ preserves $R^{+, 2}$, and so generates an extended torus to which $\Gamma$ extends.

12. The element $t_{1}={ }_{\text {def }} w_{0}(\bar{\lambda}, \nu) w_{0}(\bar{\lambda}) \theta_{H}$ preserves $R^{+, 1}$, and so generates an extended torus to which $\Gamma$ extends.

13. The action of $w_{0}(\bar{\lambda}) \theta_{H}$ preserves $R(\bar{\lambda}, \nu)$ and the system of positive roots $R^{L,+} \cup R^{R+}$ defined by $N$; so it defines an involutive automorphism of the corresponding Coxeter system $(W(\bar{\lambda}, \nu), S(\bar{\lambda}, \nu))$. The extended tori to which $\Gamma$ extends correspond to certain conjugacy classes of twisted involutions in this Coxeter system, the correspondence sending a twisted involution $x$ to $\left\langle x w_{0}(\bar{\lambda}) \theta_{H}, H\right\rangle$. 
Example 13.13. Suppose $G=S L(6, \mathbb{C})$. Choose for $H$ the subgroup of diagonal matrices, so that

$$
\mathfrak{a}^{*}=\left\{\left(\nu_{1}, \ldots, \nu_{6}\right) \in \mathbb{C}^{6} \mid \sum_{i} \nu_{i}=0\right\} .
$$

The real Weyl group is $S_{6}$, acting on $\mathfrak{a}^{*}$ by permuting the coordinates. The restrictions to $\mathfrak{a}$ of the roots are the thirty elements

$$
\left\{e_{i}-e_{j} \mid 1 \leq i \neq j \leq 6\right\} .
$$

Each of these corresponds to a single pair of roots $(\alpha,-\theta \alpha)$. We will abuse notation and call this set $R$. The upper triangular matrices constitute a Borel subgroup, corresponding to the positive system

$$
R^{+}=\left\{e_{i}-e_{j} \mid 1 \leq i<j \leq 6\right\} .
$$

Consider the Langlands parameter

$$
\Gamma=(1, \nu), \quad \nu=(1,1,0,0,-1,-1),
$$

corresponding to a spherical principal series representation. Because $\Lambda$ is trivial, $L(\bar{\lambda})=G$. We have

$$
\begin{aligned}
R^{0}(\nu) & =\left\{ \pm\left(e_{1}-e_{2}\right), \pm\left(e_{3}-e_{4}\right), \pm\left(e_{5}-e_{6}\right)\right\} \\
R^{+}(\nu) & =R^{+}-\left\{e_{1}-e_{2}, e_{3}-e_{4}, e_{5}-e_{6}\right\}
\end{aligned}
$$

So

$$
W(\bar{\lambda}, \nu)=\left\{1, s_{12}\right\} \times\left\{1, s_{34}\right\} \times\left\{1, s_{56}\right\} .
$$

The element $w_{0}(\bar{\lambda})$ is the long element of $S_{6}$, which reverses the order of the six coordinates:

$$
w_{0}(\bar{\lambda})\left(\nu_{1}, \ldots, \nu_{6}\right)=\left(\nu_{6}, \ldots, \nu_{1}\right) .
$$

Conjugation by $-w_{0}(\lambda)$ (since we are looking only at $\mathfrak{a}$, we can think of $\theta_{H}$ as -1 ) interchanges the two generators $s_{12}$ and $s_{56}$, and fixes $s_{34}$. It is easy to check that there are exactly two conjugacy classes of twisted involutions, namely

$$
\left\{1, s_{12} s_{56}\right\} \quad \text { and } \quad\left\{s_{34} s_{12}, s_{34} s_{56}\right\} .
$$

The first class corresponds to $t_{2}$ in the Proposition, and the second to $t_{1}$. 
Proof. Recall from Proposition 4.2 that complex conjugation acts on the roots by $-\theta$. Therefore a Levi subalgebra containing $\mathfrak{h}$ is real if and only if it is $\theta$-stable. That the sets $R^{0}(\nu)$ and $R^{0}(\bar{\lambda})$ are $\theta$-stable is immediate from the fact that $\theta \nu=-\nu$ and $\theta \bar{\lambda}=\bar{\lambda}$. This proves (1).

For $(2)$, the fact that $-\theta \nu=\nu$ implies that $R^{+}(\nu)$ is preserved by complex conjugation. Similarly (3) follows from $\theta \bar{\lambda}=\bar{\lambda}$.

For (4), no real coroot can vanish on $\rho_{\mathbb{R}}$; and (5) is similar. Part (6) is immediate from (1).

The first assertion in (7) follows from (4) and (5). That this forces every simple (real) factor to be a complex group follows either from the classification of real forms, or from any of the ideas leading to the proof of that classification.

Part (8) is a consequence of the analysis of the Weyl group action on Langlands parameters made in [42].

The hypothesis on $\Gamma$ is that there is an element $w \in W(G, H)$ such that $w \theta_{H} \Gamma=\Gamma ;$ so

$$
w \bar{\lambda}=\bar{\lambda}, \quad w R_{i \mathbb{R}}^{+}=R_{i \mathbb{R}}^{+}, \quad w \nu=-\nu .
$$

The first two conditions imply that $w \in W(L(\bar{\lambda}), H)$, and the last that $w \in W(\bar{\lambda}, \nu) w_{0}(\bar{\lambda})$. Now (10) follows, and the rest of (9)-(13) are elementary consequences.

Section 9 offered two constructions of standard modules from Langlands parameters, using appropriately chosen systems of positive roots. In the same way we will find two constructions of standard modules for ${ }^{\delta} G$ from extended parameters. For a type two parameters, the standard representation is simply induced from a standard representation for $G$; we will say nothing more about that case. We continue to assume for simplicity that the infinitesimal character is real, and therefore place ourselves in the situation of (13.11). Here is the first construction, based on Langlands' construction of the standard representations of $G$.

Definition 13.14. In the setting (13.11), write

$$
{ }^{1} H=\left\langle H, \delta_{1}\right\rangle, \quad t_{1}=w_{1} \theta_{H} \in{ }^{\delta} W(G, H)
$$

for the (first) extended maximal torus constructed in Proposition 13.12, and $\Gamma_{1}=\left(\Lambda_{1}, \nu\right)$ for an extended Langlands parameter extending $\Gamma$, decomposed as in Definition 6.5. Write $R_{i \mathbb{R}}^{+}$for the system of positive imaginary roots that is part of the discrete parameter $\Lambda$, and $R_{\mathbb{R}}^{+}$for a system of positive real roots making $\nu$ weakly dominant and preserved 
by $t_{1}$ (such as the one used in the proof of Proposition 13.12). Then (Lemma 13.10)

$$
t_{1} \Lambda=\Lambda, \quad t_{1} \nu=\nu, \quad t_{1} R_{i \mathbb{R}}^{+}=R_{i \mathbb{R}}^{+}, \quad t_{1} R_{\mathbb{R}}^{+}=R_{\mathbb{R}}^{+} .
$$

\{eq:extpreserve\}

Define a real parabolic subgroup

$$
P(\nu)=L(\nu) N(\nu), \quad t_{1}(P(\nu))=P(\nu)
$$

as in Proposition 13.12(2). (This parabolic subgroup is slightly smaller than the one in Langlands' original construction of standard representations, which used only $\nu$ and not also the choice of positive real roots.) We may therefore define

$$
{ }^{1} L(\nu)=\left\langle L(\nu), t_{1}\right\rangle,
$$

the group generated by $L(\nu)$ and (any representative of) $t_{1}$; we get

$$
{ }^{1} P(\nu)={ }^{1} L(\nu) N(\nu),
$$

a parabolic subgroup of ${ }^{\delta} G$. Because the imaginary roots are all in $L(\nu), \Gamma_{1}$ is still an extended parameter for ${ }^{1} L(\nu)$. We will define

$$
I_{\mathrm{quo}, G}\left(\Gamma_{1}\right)=\operatorname{Ind}_{1}^{\delta} G(\nu)\left(I_{\mathrm{quo}, L(\nu)}\left(\Gamma_{1}\right) \otimes 1\right) .
$$

(Here induction is normalized as usual.) So we are reduced to the problem of defining the extended standard representation on ${ }^{1} L(\nu)$.

The $\theta$-stable parabolic $\mathfrak{p}(\bar{\lambda})$ of Proposition $13.12(3)$ clearly meets $\mathfrak{l}(\lambda)$ in a $\theta$-stable parabolic subalgebra

$$
\mathfrak{p}(\lambda) \cap \mathfrak{l}(\nu)={ }_{\operatorname{def}} \mathfrak{p}_{\mathfrak{l}(\nu)}(\lambda)=\mathfrak{l}(\lambda, \nu)+\mathfrak{n}_{\mathfrak{l}(\nu)}(\lambda)
$$

with Levi factor $L(\lambda, \nu)$ and preserved by $t_{1}$. By the particular choice of $t_{1}$ (among all elements preserving $\Gamma$ ) there is a $\theta$-stable Borel subalgebra $\mathfrak{b}(\lambda, \nu)$ in $\mathfrak{l}(\lambda, \nu)$, containing $\mathfrak{h}$, that is also preserved by $t_{1}$. We therefore have a $t_{1}$-stable, $\theta$-stable Borel subalgebra

$$
\mathfrak{b}(\lambda, \nu)+\mathfrak{n}_{\mathfrak{l}(\nu)}(\lambda)={ }_{\text {def }} \mathfrak{b}_{\mathfrak{l}(\nu)}=\mathfrak{h}+\mathfrak{n}_{\mathfrak{l}(\nu)}
$$

with Levi subgroup $H$. Recall that $\Lambda_{1}$ involves a genuine character $\lambda_{1}$ of the $\rho_{i \mathbb{R}}$ cover of ${ }^{1} T$. Following $(9.3 \mathrm{~g})$, we define a character

$$
\lambda_{1, \mathfrak{b}_{\mathfrak{l}(\nu)}}=\left[\lambda_{1}-\rho_{i \mathbb{R}}\right]-2 \rho\left(\mathfrak{n}_{\mathfrak{l}(\nu)} \cap \mathfrak{k}\right)+2 \rho_{c, i \mathbb{R}}
$$


of ${ }^{1} T$. Here the first term in brackets is a character of ${ }^{1} T$ by Definition 5.1 ; the second, thought of as the "sum of the compact positive roots," means the character of ${ }^{1} T$ on the top exterior power of the indicated nilpotent subalgebra; and the third is the character on the top exterior power of the span of the compact imaginary roots. (We cannot just say "sum of roots," because the extended torus ${ }^{1} T$ may permute the root spaces.)

Following Theorem 9.4, we use cohomological induction to define

$$
I_{\text {quo }, L(\nu)}\left(\Gamma_{1}\right)=\left(\mathcal{L}_{\mathfrak{b}_{\mathfrak{l}(\nu)}^{\mathrm{l}(\nu),},{ }^{1}(L(\nu) \cap K)}\right)^{s}\left(\Gamma_{1, \mathfrak{b}_{\mathrm{l}(\nu)}}\right)
$$

Here $s=\operatorname{dim}\left(\mathfrak{n}_{\mathfrak{l}(\nu)} \cap \mathfrak{k}\right)$. A little more explicitly, what this definition means is this.

1. The representation $I_{\text {quo }, L(\nu)}\left(\Gamma_{1}\right)$ contains the ${ }^{1}(L(\nu) \cap K)$ representation of highest ${ }^{1} T$-weight (with respect to $\mathfrak{n}_{\mathfrak{l}(\nu)} \cap \mathfrak{k}$ )

$$
\mu_{1}\left(\Gamma_{1}\right)=\left[\lambda_{1}+\rho_{i \mathbb{R}}\right]-2 \rho_{c, i \mathbb{R}} .
$$

2. Equivalently, the weight

$$
\mu_{1}\left(\Gamma_{1}\right)-2 \rho\left(\mathfrak{n}_{\mathfrak{l}(\nu)} \cap \mathfrak{k}\right)=\lambda_{1, \mathfrak{b}_{\mathfrak{l}(\nu)}}+2 \rho\left(\mathfrak{n}_{\mathfrak{l}(\nu)}\right)
$$

appears in $H_{s}\left(\mathfrak{n}_{\mathfrak{l}(\nu)}^{\text {op }} \cap \mathfrak{k}, I_{\text {quo }, L(\nu)}\left(\Gamma_{1}\right)\right)$.

3. The restriction map

$$
H_{s}\left(\mathfrak{n}_{\mathfrak{l}(\nu)}^{\mathrm{op}}, I_{\mathrm{quo}, L(\nu)}\left(\Gamma_{1}\right)\right) \rightarrow H_{s}\left(\mathfrak{n}_{\mathfrak{l}(\nu)}^{\mathrm{op}} \cap \mathfrak{k}, I_{\mathrm{quo}, L(\nu)}\left(\Gamma_{1}\right)\right)
$$

is an isomorphism on the ${ }^{1} T$-weight space in question; and

4. The extended torus ${ }^{1} H$ acts on this ${ }^{1} T$-weight space by the character $\Gamma_{1, \mathfrak{b}_{\mathfrak{l}(\nu)}}+2 \rho\left(\mathfrak{n}_{\mathfrak{l}(\nu)}\right)$.

Here is the corresponding construction emphasizing instead cohomological induction.

Definition 13.15. In the setting (13.11), write

$$
{ }^{2} H=\left\langle H, \delta_{2}\right\rangle, \quad t_{2}=w_{2} \theta_{H} \in{ }^{\delta} W(G, H)
$$

for the (second) extended maximal torus constructed in Proposition 13.12, and $\Gamma_{2}=\left(\Lambda_{2}, \nu\right)$ for an extended Langlands parameter extending 
$\Gamma$, decomposed as in Definition 6.5. Write $R_{i \mathbb{R}}^{+}$for the system of positive imaginary roots that is part of the discrete parameter $\Lambda$, and $R_{\mathbb{R}}^{+}$for a system of positive real roots making $\nu$ weakly dominant and preserved by $t_{2}$ (such as the one used in the proof of Proposition 13.12). Then (Lemma 13.10)

$$
t_{1} \Lambda=\Lambda, \quad t_{1} \nu=\nu, \quad t_{1} R_{i \mathbb{R}}^{+}=R_{i \mathbb{R}}^{+}, \quad t_{1} R_{\mathbb{R}}^{+}=R_{\mathbb{R}}^{+} .
$$

Define a $\theta$-stable parabolic subalgebra

$$
\mathfrak{p}(\bar{\lambda})=\mathfrak{l}(\bar{\lambda})+\mathfrak{n}(\bar{\lambda}), \quad t_{2}(\mathfrak{p}(\bar{\lambda}))=\mathfrak{p}(\bar{\lambda})
$$

as in Proposition 13.12(3). (This parabolic subalgebra is slightly smaller than the one used in [38] to construct standard representations; that one used only $\bar{\lambda}$ and not also the system of positive imaginary roots.) We may therefore define

$$
{ }^{2} L(\bar{\lambda})=\left\langle L(\bar{\lambda}), t_{2}\right\rangle
$$

the group generated by $L(\bar{\lambda})$ and (any representative of) $t_{2}$.

Just as in $(9.3 \mathrm{~g})$, the extended parameter $\Gamma_{2}$ for $G$ defines an extended parameter $\Gamma_{2, \mathfrak{p}(\bar{\lambda})}$ for ${ }^{1} L(\bar{\lambda})$. The cohomological induction construction of the extended standard module is

$$
I_{\mathrm{quo}, G}\left(\Gamma_{2}\right)=\left(\mathcal{L}_{\mathfrak{p}^{\mathrm{op}}(\bar{\lambda}),{ }^{2} L(\bar{\lambda}) \cap^{\delta} K}^{\mathfrak{g}^{\delta} K}\right)^{s}\left(I_{\mathrm{quo}, L(\bar{\lambda})}\left(\Gamma_{2, \mathfrak{p}(\bar{\lambda})}\right)\right) .
$$

$\{$ eq:VZextind $\}$

Here $s=\operatorname{dim}(\mathfrak{n}(\bar{\lambda}) \cap \mathfrak{k})$, which is different from (generally larger than) the $s$ appearing in Definition 13.14.

What remains is to construct the standard module $I_{\text {quo }, L(\bar{\lambda})}\left(\Gamma_{2, \mathfrak{p}(\bar{\lambda})}\right)$ for the group ${ }^{2} L(\lambda)$. But this is easy: we saw in Proposition 13.12(5) that $L(\bar{\lambda})$ is quasisplit, with a Borel subgroup $B=H N$ making $\nu$ weakly dominant. The element $t_{2}$ was constructed to satisfy

$$
t_{2}(N)=N,
$$

so we simply define ${ }^{2} B={ }^{2} H N$, and

$$
I_{\text {quo }, L(\bar{\lambda})}\left(\Gamma_{2, \mathfrak{p}(\bar{\lambda})}\right)=\operatorname{Ind}_{2}^{2} L(\bar{\lambda})\left(\Gamma_{2, \mathfrak{p}(\bar{\lambda})}\right) .
$$

That this representation extends

$$
I_{\text {quo }, L(\bar{\lambda})}\left(\Gamma_{\mathfrak{p}(\bar{\lambda})}=\operatorname{Ind}_{B}^{L(\bar{\lambda})}\left(\Gamma_{\mathfrak{p}(\bar{\lambda})}\right)\right.
$$

is immediate. 
$\{$ thm : extLC $\}$

Theorem 13.16 (Langlands classification for extended groups). Suppose that $G$ is the group of real points of a connected complex reductive algebraic group (cf. (3.1)), and that ${ }^{\delta} G$ is a corresponding extended group (cf. (12.1)). Then there is a one-to-one correspondence between infinitesimal equivalence classes of irreducible quasisimple representations of ${ }^{\delta} G$ (Definition 2.14) and equivalence classes of extended Langlands parameters for ${ }^{\delta} G$ (Definition 13.7). In this correspondence, type one representations (those restricting irreducibly to $G$ ) correspond to one-dimensional parameters; and type two representations (those induced irreducibly from $G$ ) correspond to twodimensional parameters.

This is a consequence of the Langlands classification for the connected group (Theorem 6.1), of Clifford theory (Proposition 8.13), and of the analysis of extended parameters made in Proposition 13.12 above. Of course one wants to know that the two constructions offered above lead to exactly the same standard representations (the alternative being that the two extensions to ${ }^{\delta} G$ are sometimes interchanged). This can be proven in the same way as the equivalence of the constructions in Theorems 9.2 and 9.4 above ([21], Theorem 11.129). We omit the details.

\section{Jantzen filtrations and Hermitian forms}

For the rest of this paper we will need to work with extended groups and $\{\sec : j$ antzen $\}$ their representations, in order to make use ultimately of the description of invariant Hermitian forms in Theorem 12.9. In some cases, as in the present section, the extension from $G$ to ${ }^{\delta} G$ is entirely routine and obvious. In such cases we may formulate results just for $G$, in order to keep the notation a little simpler.

Character theory for Harish-Chandra modules is based on expressing the (complicated) characters of irreducible modules as (complicated) integer combinations of the (relatively simple) characters of standard modules. We want to do something parallel for signatures of invariant forms: to express the invariant forms on irreducible modules as integer combinations of forms on standard modules. A fundamental obstruction to this plan is part (3) of Propositions 10.4 and 10.7: if the Langlands quotient is proper, then the standard module $I(\Gamma)$ cannot admit a nondegenerate invariant Hermitian form.

Jantzen (in [16], 5.1) developed tools to deal with this obstruction; what we need is contained in Definition 14.9 below. The idea is that each Lang- 
lands parameter $\Gamma=(\Lambda, \nu)$ (Definition 6.5) is part of a natural family of parameters

$$
\Gamma_{t}={ }_{\text {def }}(\Lambda, t \nu) \quad(0 \leq t<\infty) .
$$

$\{$ eq: Gammat $\}$

For $t>0$, it is evident that $\Gamma_{t}$ inherits from $\Gamma$ the formal defining properties of a Langlands parameter. At $t=0$, all the real roots satisfy $\left\langle d \gamma, \alpha^{\vee}\right\rangle=0$, and condition 5 of Theorem 6.1 can fail; $\Gamma_{0}$ may be only a weak Langlands parameter (Definition 6.3). It is a classical idea (originating in the definition of complementary series representations for $S L(2)$ by Bargmann and Gelfand-Naimark, and enormously extended especially by Knapp) that one can study questions of unitarity by deformation arguments in the parameter $t$. In order to do that, we need to know that the family of representations indexed by $\Gamma_{t}$ is "nice." (The parameter $t$ may be allowed to vary over all of $\mathbb{R}$ or $\mathbb{C}$ for many of the statements to follow; this causes difficulties only with references to the "quotient" realization of a standard module, or to invariant Hermitian forms. In a similar way, we could allow the continuous parameter to vary over all of $\mathfrak{a}^{*}$, rather than just over the line $\mathbb{R} \nu$, with similar caveats.)

Proposition 14.2 (Knapp-Stein [23], Theorem 6.6). If $\Gamma=(\Lambda, \nu)$ is a \{prop:meromorphic\} Langlands parameter, then all of the (weak) standard modules (for $t \geq 0$ ) $I_{\text {quo }}\left(\Gamma_{t}\right)$ and $I_{\mathrm{sub}}\left(\Gamma_{t}\right)$ (cf. (14.1)) may be realized on a common space

$$
V(\Lambda)={ }_{\operatorname{def}} \operatorname{Ind}_{M \cap K}^{K}\left(\left.D(\Lambda)\right|_{M \cap K}\right)
$$

(notation as in (6.4)). In this realization

1. the action of the group $K$ (and therefore of the Lie algebra $\mathfrak{k}$ ) is independent of $t$.

2. The action of the Lie algebra $\mathfrak{g}$ in $I_{\mathrm{quo}}\left(\Gamma_{t}\right)$ depends in an affine way on $t$. That is, for each $X$ in $\mathfrak{g}$ there are linear operators $Q_{0}(X)$ and $Q_{1}(X)$ on $V(\Lambda)$ so that the action of $X$ in $I_{\text {quo }}\left(\Gamma_{t}\right)$ is $Q_{0}(X)+t Q_{1}(X)$. When $V(\Lambda)$ is interpreted as a space of functions on $K$, the operators $Q_{0}(X)$ are first-order differential operators, and the operators $Q_{1}(X)$ are multiplication operators.

3. The action of the Lie algebra $\mathfrak{g}$ in $I_{\text {sub }}\left(\Gamma_{t}\right)$ depends in an affine way on t. That is, for each $X$ in $\mathfrak{g}$ there are linear operators $S_{0}(X)$ and $S_{1}(X)$ on $V(\Lambda)$ so that the action of $X$ in $I_{\text {quo }}\left(\Gamma_{t}\right)$ is $S_{0}(X)+t S_{1}(X)$. When $V(\Lambda)$ is interpreted as a space of functions on $K$, the operators $S_{0}(X)$ are first-order differential operators, and the operators $S_{1}(X)$ are multiplication operators. 
For $t>0$, there is a nonzero interwining operator

$$
L_{t}=L_{t}(\Gamma): I_{\mathrm{quo}}\left(\Gamma_{t}\right) \rightarrow I_{\mathrm{sub}}\left(\Gamma_{t}\right),
$$

unique up to a nonzero scalar multiple. Fix a lowest $K$-type $\mu$ of $J(\Gamma)$ (equivalently, a lowest $K$-type of $V(\lambda)$ ) and normalize $L_{t}$ by the requirement

$$
\left.L_{t}\right|_{\mu}=\mathrm{Id} .
$$

4. The intertwining operator $L_{t}$ is a rational function of $t$ (that is, each matrix entry is a quotient of polynomials) having no poles on $[0, \infty)$.

5. The limit operator $L_{0}$ is a unitary intertwining operator between two unitarily induced representations.

6. Suppose that $J(\Gamma)$ admits a nonzero invariant or c-invariant Hermitian form $\langle,\rangle_{1}$. Regard this as a form on $I_{\text {quo }}(\Gamma)$ with radical equal to the maximal submodule, and therefore as a form on $V(\Lambda)$. Then this form has a canonical extension to a rational family of forms $\langle,\rangle_{t}$ on $V(\Lambda)$, characterized by the two requirements

(a) for $t>0$, the form $\langle,\rangle_{t}$ is invariant for $I_{\mathrm{quo}}\left(\Gamma_{t}\right)$; and

(b) the form $\langle,\rangle_{t}$ is constant on the fixed lowest $K$-type $\mu$.

The family of forms also satisfies

(c) the form $\langle,\rangle_{t}$ has no poles on $[0, \infty)$; and

(d) for $t>0$, the radical of $\langle,\rangle_{t}$ is equal to the maximal proper submodule, so that the form descends to a nondegenerate form on the Langlands quotient $J\left(\Gamma_{t}\right)$.

Outline of proof. We will not give a detailed argument; but since the results about rational dependence on $t$ are so fundamental to our unitarity algorithm, we will recall briefly the reasoning. Of course the statement about a common space is part of Theorem 6.1; and the description of that space is a consequence of the decomposition $G=K M A N$. (This is closely related to the Iwasawa decomposition of $G$, but not the same, since $M A N$ is not a minimal parabolic subgroup of $G$. If one begins with a minimal parabolic subgroup $M_{1} A_{1} N_{1}$ of $M$, then $M_{1}\left(A_{1} A\right)\left(N_{1} N\right)$ is a minimal parabolic in $G$, so $G=K\left(A_{1} A\right)\left(N_{1} N\right)$ is an Iwasawa decomposition. Combining this with the Iwasawa decomposition $M=(M \cap K) A_{1} N_{1}$ of $M$ leads to the formulas

$$
G=K M A N, \quad G / M A N \simeq K / M \cap K
$$


and then immediately to the description of $V(\Lambda)$.) Part (1) of the theorem is now obvious.

For part (2), write $\lambda$ and $\rho$ for the actions of $\mathfrak{g}$ on functions on $G$ by differentiation on the left or the right respectively. To make an element $X \in \mathfrak{g}$ act on a function $f_{0} \in V(\Lambda)$ - that is, a function on $K$ with values in the space of $\Lambda$, transforming on the right under $M \cap K$-we must first extend $f_{0}$ to a function $f_{\nu}$ on all of $G$, with an appropriate transformation on the right under $M A N$. The action of $X$ on $f_{\nu}$ is on the left, by $\lambda(X)$. To calculate it, we use the formula

$$
\lambda(X)(k)=\rho\left(\operatorname{Ad}\left(k^{-1}\right) X\right)(k) .
$$

To compute the action of $\rho\left(\operatorname{Ad}\left(k^{-1}\right) X\right)$ on $f_{\nu}$, we compute the (unique) decomposition

$$
\operatorname{Ad}\left(k^{-1}\right) X=\kappa(k, X)+\mu(k, X)+\alpha(k, X)+\beta(k, X) \in \mathfrak{k}+\mathfrak{m} \cap \mathfrak{s}+\mathfrak{a}+\mathfrak{n} .
$$

Here $\mathfrak{s}$ is the -1 eigenspace of the Cartan involution. The action of $\beta(k, X)$ is zero by definition, and that of $\kappa(k, X)$ and $\mu(k, X)$ is (almost obviously) independent of $\nu$. The action of $\alpha(k, X)$ is multiplication by the smooth function

$$
(\nu+\rho)(\alpha(k, X))
$$

which depends in an affine way on $\nu$. This proves (2). Part (3) is identical, with $N$ replaced by $N^{\text {op }}$.

The existence and uniqueness of the intertwining operator $L_{t}$ is part of the Langlands classification (Theorem 6.1). Once the normalization is made, we can calculate $L_{t}$ in the following way. Fix any representation $\left(\mu_{1}, E_{1}\right)$ of $K$, having multiplicity $n$ in $V(\Lambda)$. Pick any one-dimensional subspace $S_{1}$ in $E_{1}$, and choose a basis $\left\{v_{1}, \ldots, v_{n}\right\}$ for the corresponding subspace of $V(\Lambda)$. Pick a one-dimensional subspace $S_{0}$ of the lowest $K$-type $\mu$, and a basis vector $v_{0}$ for the corresponding line in $V(\Lambda)$. Because $I(\Gamma)$ has a unique irreducible quotient representation $J(\Gamma)$ that contains $\mu$, the vector $v_{0}$ necessarily generates $I(\Gamma)$ as a module over the Hecke algebra $R\left(\mathfrak{g}_{0}, K\right)$ (see [21], Chapter 1). So we can find elements $r_{i} \in R\left(\mathfrak{g}_{0}, K\right)$ with

$$
r_{i} \cdot v_{0}=v_{i} \quad(\text { in } I(\Gamma)) .
$$

$\{$ eq: cyclic $\}$

Now write $\delta_{0} \in R(K)$ for a projection operator on $E_{0}$ and $\delta_{1}$ for a projection on $E_{1}$. This means

$$
\tau\left(\delta_{0}\right)= \begin{cases}\text { projection on } E_{0} & \left(\tau=\mu_{0}\right) \\ 0 & \left(\tau \neq \mu_{0}\right),\end{cases}
$$


(with $\tau$ some irreducible representation of $K$ ); and similarly for $\delta_{1}$. (Such elements can be found because the Hecke algebra of $K$ may be identified with the sum of the endomorphism algebras of all the irreducible representations of $K$.) Then

$$
\begin{gathered}
\delta_{0} \cdot v_{0}=v_{0}, \quad \delta_{0} \cdot V(\Lambda)=\left\langle v_{0}\right\rangle, \\
\delta_{1} \cdot v_{i}=v_{i}, \quad \delta_{i} \cdot V(\Lambda)=\left\langle v_{1}, \ldots, v_{n}\right\rangle .
\end{gathered}
$$

For any value of $t$, the element

$$
\widetilde{r}_{j}=\delta_{1} r_{j} \delta_{0}
$$

of $R\left(\mathfrak{g}_{0}, K\right)$ carries $v_{0}$ to the span of the $v_{i}$; so we can define an $n \times n$ matrix-valued function $Q$ of $t$ by

$$
\tilde{r}_{j} \cdot v_{0}=\sum_{i=1}^{n} Q_{i j}(t) v_{i} \quad\left(\text { in } I_{\mathrm{quo}}\left(\Gamma_{t}\right)\right.
$$

(The letter $Q$ refers to the "quotient" standard module.) According to part (2) of the theorem, the entries $Q_{i j}$ are polynomial functions of $t$, of degree bounded by the degree of the enveloping algebra elements appearing in $r_{j}$. According to (14.4), $Q(1)$ is the identity matrix.

In exactly the same way, we can define an $n \times n$ matrix $S(t)$ by

$$
\widetilde{r}_{j} \cdot v_{0}=\sum_{i=1}^{n} S_{i j}(t) v_{i} \quad\left(\text { in } I_{\mathrm{sub}}\left(\Gamma_{t}\right) .\right.
$$

Again we find that the entries of $S$ are polynomial functions of $t$.

The intertwining operator $L(t)$ respects the action of $K$, so preserves the span of the $v_{i}$; so we can define an $n \times n$ matrix $M(t)$ by

$$
L(t) \cdot v_{i}=\sum_{k=1}^{n} M_{k i}(t) v_{k} .
$$

The intertwining property of $L(t)$ means that

$$
L(t) \cdot \widetilde{r}_{j} \cdot v_{0}=\widetilde{r}_{j} \cdot L(t) \cdot v_{0} .
$$

By the normalization of $L(t), L(t) \cdot v_{0}=v_{0}$; so this last equation is equivalent to the matrix equation

$$
M(t) Q(t)=S(t)
$$


Because $Q(1)$ is the identity, $Q(t)^{-1}$ is a rational matrix-valued function of $t$, with no poles near $t=1$; so

$$
M(t)=S(t) Q(t)^{-1}
$$

has the same property. This argument proves (4), except for the assertion that there is no pole at zero.

To deal with the possible pole at zero, and to prove (5), it is helpful to vary the continuous parameter not just along the line $\mathbb{R} \nu$ but in all of $\mathfrak{a}^{*}$. The (normalized) intertwining operator $L\left(\nu^{\prime}\right)$ is a rational function of $\nu^{\prime}$, with no poles in some positive open cone: the matrix entries are quotients of polynomial functions of $\nu^{\prime}$. We fix a real-valued and regular linear functional $\rho$, and we let $\nu^{\prime}$ approach not zero but the small purely imaginary character $i \epsilon \rho$. In this case the limit representation $I(\Lambda, i \epsilon \rho)$ is unitary and irreducible, so the limiting intertwining operator $L(i \epsilon \rho)$ must act by a positive real scalar times a unitary operator. Since $L$ was normalized to be one on a fixed lowest $K$-type, this scalar must be equal to one: so $L(i \epsilon \rho)$ is unitary. Taking the limit as $\epsilon \rightarrow 0$ gives (5). The absence of the pole at zero follows as well.

Part (6) is a formal consequence of these facts about $L(t)$ and the description of invariant forms in Propositions 10.4 and 10.7.

We are going to use the rational family of intertwining operators $L(t)$ $\{$ se: jantzenfilt $\}$ to analyze the submodule structure of $I_{\mathrm{quo}}(\Gamma)$. We have already seen that defining

$$
I_{\text {quo }}(\Gamma)^{0}==_{\text {def }} I_{\text {quo }}(\Gamma), \quad I_{\text {quo }}(\Gamma)^{1}={ }_{\text {def }} \operatorname{ker}(L(1))
$$

has the consequence

$$
I_{\mathrm{quo}}(\Gamma)^{0} / I_{\mathrm{quo}}(\Gamma)^{1} \simeq J(\Gamma) .
$$

Once we have decided that the family of operators $L(t)$ is interesting, it is natural to try to define

$$
I_{\text {quo }}(\Gamma)^{2} \stackrel{?}{=}\{v \in V(\Lambda) \mid L(t) v \text { vanishes to order two at } t=1\} \subset I_{\text {quo }}(\Gamma)^{1} .
$$

The difficulty is that this space need not be a submodule. Here is why. Suppose that $L(t) v$ vanishes to second order, and $x$ is in the Hecke algebra $R\left(\mathfrak{g}_{0}, K\right)$. Write $\cdot t$ for the action in $I_{\text {quo }}\left(\Gamma_{t}\right)$. We would like to show that $L(t)[x \cdot 1 v]$ vanishes to second order at $t=1$. The natural way to do that would be to interchange $x$ and $L(t)$. But this we are not permitted to do: the correct intertwining relation is rather

$$
L(t)\left[x \cdot{ }_{t} v\right]=x \cdot{ }_{t}[L(t) v] .
$$


The correct definition of the Jantzen filtration is

$$
I_{\text {quo }}(\Gamma)^{r}=\left\{\begin{array}{ll}
v \in V(\Lambda) \mid \begin{array}{l}
\text { for some rational function } f_{v}(t) \text { with } \\
f_{v}(1)=v, L(t) f_{v}(t) \text { vanishes to order } \\
r \text { at } t=1 .
\end{array}
\end{array}\right\}
$$

$\{$ eq:jantzenr $\}$

It is easy to check that $f_{v}$ can be taken to be a polynomial of degree at most $r-1$ without changing the space defined. Another way to write the definition is

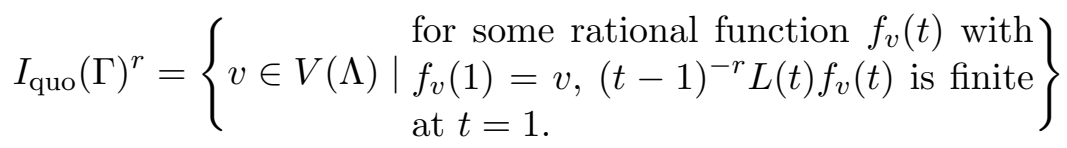

Now the invariance of $I_{\text {quo }}(\Gamma)^{r}$ under the Hecke algebra is easy: if $f_{v}$ is the rational function of $t$ certifying the membership of $v$ in $I_{\text {quo }}(\Gamma)^{r}$, and $x$ belongs to the Hecke algebra, then we can define

$$
f_{x \cdot 1 v}(t)={ }_{\text {def }} x \cdot{ }_{t} f_{v}(t),
$$

which is a rational function of $t$ taking the value $x \cdot{ }_{1} v$ at $t=1$. Furthermore

$$
L(t)\left[f_{x \cdot 1 v}(t)\right]=L(t)\left[x \cdot{ }_{t} f_{v}(t)\right]=x \cdot{ }_{t}\left[L(t) f_{v}(t)\right] .
$$

The function $\left[L(t) f_{v}(t)\right]$ is assumed to vanish to order $r$ at $t=1$, so this is true after we apply the operator-valued polynomial function $x \cdot{ }_{t}$. That is, the function $f_{x \cdot 1}$ shows $x \cdot 1 v$ also belongs to $I_{\text {quo }}(\Gamma)^{k}$.

The Jantzen filtration is the decreasing filtration by submodules

$$
I_{\text {quo }}(\Gamma)=I_{\text {quo }}(\Gamma)^{0} \supset I_{\text {quo }}(\Gamma)^{1} \supset I_{\text {quo }}(\Gamma)^{2} \supset \cdots
$$

Because the image of the intertwining operator is the Langlands quotient, we get

$$
I_{\text {quo }}(\Gamma)^{1}=\operatorname{ker} L(1), \quad I_{\text {quo }}(\Gamma)^{0} / I_{\text {quo }}(\Gamma)^{1} \simeq J(\Gamma) .
$$

Since we are talking about rational functions, (14.5d) could formally be extended to negative values of $k$, using order of pole rather than order of vanishing. Since $L(t)$ has no poles for $t>0$, such a definition adds nothing for $I_{\text {quo }}(\Gamma)$. But it is perfectly reasonable to consider the inverse intertwining operator

$$
L(t)^{-1}: I_{\mathrm{sub}}\left(\Gamma_{t}\right) \rightarrow I_{\mathrm{quo}}\left(\Gamma_{t}\right)
$$


(always on our fixed space $V(\Lambda)$.) Again the matrix coefficients are rational functions of $t$, but now there can be lots of poles in $(0, \infty)$. For $r \geq 0$, we define

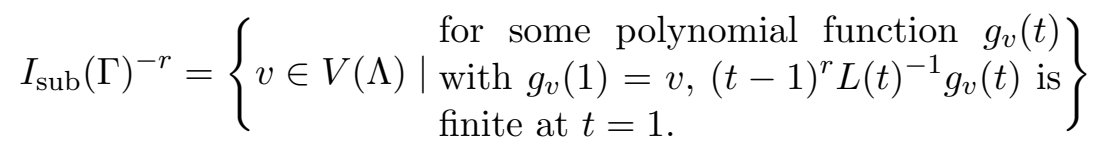

$\{$ eq: jantzenminus $\}$

On this side the Jantzen filtration is

$$
0=I_{\mathrm{sub}}(\Gamma)^{1} \subset I_{\mathrm{sub}}(\Gamma)^{0} \subset I_{\mathrm{sub}}(\Gamma)^{-1} \subset \cdots \subset I_{\mathrm{sub}}(\Gamma) .
$$

The Langlands subrepresentation is

$$
I_{\text {sub }}(\Gamma)^{0}=\operatorname{im} L(1), \quad I_{\text {sub }}(\Gamma)^{0} / I_{\text {sub }}(\Gamma)^{1} \simeq J(\Gamma) .
$$

Lemma 14.6 (Jantzen [16], Lemma 5.1). Suppose $E$ and $F$ are finitedimensional vector spaces over a field $k$, and

$$
L \in k(t) \otimes_{k} \operatorname{Hom}_{k}(E, F) \simeq \operatorname{Hom}_{k(t)}\left(k(t) \otimes_{k} E, k(t) \otimes_{k} F\right) .
$$

is a rational family of linear maps from $E$ to $F$. Assume that $L$ is invertible over $k(t)$; equivalently, that $\operatorname{dim}_{k} E=\operatorname{dim}_{k} F$, and that in any bases for $E$ and $F$, the representation of $L$ as a matrix of rational functions has determinant a nonzero rational function. Define

$$
\begin{gathered}
E^{r}(1)=E^{r}=\left\{\begin{array}{l}
\text { for some rational function } f_{v}(t) \\
v \in E \mid \begin{array}{l}
\text { with } f_{v}(1)=v,(t-1)^{-r} L(t) f_{v}(t) \\
\text { is finite at } t=1 .
\end{array}
\end{array}\right\} \\
F^{q}(1)=F^{q}=\left\{\begin{array}{l}
\text { for some rational function } g_{w}(t) \text { with } \\
w \in F \mid \begin{array}{l}
g_{w}(1)=w,(t-1)^{-q} L(t)^{-1} g_{w}(t) \text { is } \\
\text { finite at } t=1 .
\end{array}
\end{array}\right\}
\end{gathered}
$$

1. These are finite decreasing filtrations of $E$ and $F$, with

$$
E^{R}=0, \quad E^{-R}=E, \quad \text { all } R \text { sufficiently large, }
$$

and similarly for $F$.

2. Taking residues defines natural isomorphisms

$$
L^{[r]}(1)=L^{[r]}: E^{r} / E^{r+1} \stackrel{\sim}{\rightarrow} F^{-r} / F^{-r+1}, \quad L^{[r]} v=(t-1)^{-r} L(t) f_{v}(t)
$$

with $f_{v}$ as in the definition of $E^{r}$. The inverse is $\left(L^{-1}\right)^{[-r]}$. 
3. Define an integer $D$ by

$$
\operatorname{det}(L)=(t-1)^{D}(\text { rational function finite and nonzero at } t=1) .
$$

Then

$$
D=\sum_{r=-\infty}^{\infty} r \operatorname{dim} E^{r} / E^{r+1} .
$$

4. Suppose that $L$ is finite at $t=1$. Then $E^{-r}=E$ for all $r \geq 0$, and

$$
D=\sum_{r=1}^{\infty} \operatorname{dim} E^{r} .
$$

We will have no occasion to make use of (3), but Jantzen made it a very powerful tool, so we include the statement. In fact Jantzen looked only at the case when $L$ is finite at $t=1$, and wrote the result in the form (4); so we include that version as well.

Sketch of proof. We can multiply $L$ by a power of $(t-1)^{c}$ to ensure that $L$ is finite at $t=1$. (This shifts all the filtrations by $c$, and multiplies $\operatorname{det} L$ by $(t-1)^{c \cdot \operatorname{dim} E}$, and so shifts $D$ by $c \cdot \operatorname{dim} E$. All the conclusions of the theorem are essentially unchanged.) Now consider the local ring $A$ of rational functions finite at $t=1$. The ring $A$ is a unique factorization PID, and $t-1$ is the unique prime element. Look at the free $A$ modules

$$
M=A \otimes_{k} E, \quad M^{\prime}=A \otimes_{k} F .
$$

The operator $L$ amounts to an injective module map

$$
L: M \hookrightarrow M^{\prime} .
$$

The module $M^{\prime} / L M$ is finitely generated, so the elementary divisor theorem provides a basis $\left\{m_{1}^{\prime}, \ldots, m_{n}^{\prime}\right\}$ of $M^{\prime}$ and nonnegative integers

$$
d_{1} \leq d_{2} \leq \cdots \leq d_{n}
$$

so that

$$
\left\{(t-1)^{d_{1}} m_{1}^{\prime}, \ldots,(t-1)^{d_{n}} m_{n}^{\prime}\right\}
$$

is a basis of $L M$. Since $L$ is injective, the elements

$$
m_{i}={ }_{\text {def }} L^{-1}\left((t-1)^{d_{i}} m_{i}^{\prime}\right)
$$


are automatically a basis of $M$. Working in these bases, it is easy to show that

$$
E^{r}=\text { span of values at } 1 \text { of }\left\{m_{i} \mid d_{i} \geq r\right\},
$$

and

$$
\operatorname{det} L=(t-1)^{\sum_{i=1}^{n} d_{i}}(\text { unit in } A) .
$$

The assertions of the lemma are now easy to check. (For example, in the bases $\left\{m_{i}\right\}$ and $\left\{m_{i}^{\prime}\right\}, L$ is diagonal with the entry $(t-1)^{d}$ appearing $\operatorname{dim}\left(E^{d} / E^{d+1}\right)$ times.)

Proposition 14.7 ([43], Theorem 3.8). Suppose $\Gamma=(\Lambda, \nu)$ is a Langlands parameter, and $\Gamma_{t}=(\Lambda, t \nu)(0<t<\infty)$. Write

$$
L_{t}: I_{\mathrm{quo}}\left(\Gamma_{t}\right) \rightarrow I_{\mathrm{sub}}\left(\Gamma_{t}\right)
$$

for the rational family of Knapp-Stein intertwining operators constructed in Theorem 14.2. Define Jantzen filtrations (of submodules)

$$
I_{\text {quo }}(\Gamma)=I_{\text {quo }}(\Gamma)^{0} \supset I_{\text {quo }}(\Gamma)^{1} \supset I_{\text {quo }}(\Gamma)^{2} \supset \cdots,
$$

and

$$
0=I_{\mathrm{sub}}(\Gamma)^{1} \subset I_{\mathrm{sub}}(\Gamma)^{0} \subset I_{\mathrm{sub}}(\Gamma)^{-1} \subset \cdots
$$

as in (14.5).

1. Each Jantzen filtration is finite: for $R$ sufficiently large, $I_{\text {quo }}(\Gamma)^{R}=0$, and $I_{\mathrm{sub}}(\Gamma)^{-R}=I_{\mathrm{sub}}(\Gamma)$.

2. The Langlands quotient is

$$
\begin{aligned}
I_{\mathrm{quo}}(\Gamma) / \operatorname{ker}\left(L_{1}\right) & =I_{\mathrm{quo}}(\Gamma)^{0} / I_{\mathrm{quo}}(\Gamma)^{1} \simeq J(\Gamma) \\
& \simeq I_{\mathrm{sub}}(\Gamma)^{0} / I_{\mathrm{sub}}(\Gamma)^{1}=\operatorname{im} L_{1} /\{0\} .
\end{aligned}
$$

3. The residual operators (Lemma 14.6(2)) define $\left(\mathfrak{g}_{0}, K\right)$-module isomorphisms

$$
L^{[r]}: I_{\text {quo }}(\Gamma)^{r} / I_{\text {quo }}(\Gamma)^{r+1} \stackrel{\sim}{\longrightarrow} I_{\text {sub }}(\Gamma)^{-r} / I_{\text {sub }}(\Gamma)^{-r+1} .
$$

4. Suppose that $J(\Gamma)$ admits a nonzero invariant or c-invariant Hermitian form $\langle,\rangle_{1}$. Extend this to a rational family of invariant forms $\langle,\rangle_{t}$ as in Proposition 14.2. Then there are nondegenerate invariant forms

$$
\langle,\rangle^{[r]} \text { on } I_{\text {quo }}(\Gamma)^{r} / I_{\text {quo }}(\Gamma)^{r+1},
$$


defined by

$$
\langle v, w\rangle^{[r]}=\lim _{s \rightarrow 1}(s-1)^{-r}\left\langle f_{v}(s), f_{w}(s)\right\rangle_{s} \quad\left(\begin{array}{l}
v, w \in I_{\mathrm{quo}}(\Gamma)^{r}, f_{v} \\
f_{w} \text { as in }(14.5 \mathrm{~d}) .
\end{array}\right)
$$

Sketch of proof. For the finiteness of the filtrations, recall that $I(\Gamma)$ has a finite number $N$ of irreducible composition factors. Choose some irreducible representation $\mu_{i}$ of $K(1 \leq i \leq N)$ appearing in each of these composition factors, and let $E \subset V(\Lambda)$ be the sum of the $\mu_{i}$-isotypic subspaces, a finitedimensional space. By the choice of the $\mu_{i}$,

$$
\begin{aligned}
& \text { each nonzero }\left(\mathfrak{g}_{0}, K\right) \text {-submodule of } I_{\text {quo }}(\Gamma) \\
& \text { has nonzero intersection with } E \text {. }
\end{aligned}
$$

Because they respect the action of $K$, the intertwining operators $L_{t}$ must preserve $E$. If we apply Lemma 14.6 to the restrictions of $L_{t}$ to $E$, we conclude that (for large $R$ ), $I_{\text {quo }}(\Gamma)^{R} \cap E=0$. By (14.8), it follows that $I_{\text {quo }}(\Gamma)^{R}=0$. The rest of (1) is similar. Part (2) is part of the Langlands classification Theorem 6.1. Part (3) is immediate from Lemma 14.6, applied separately to each (finite-dimensional) $K$-isotypic subspace.

For part (4), invariant forms are described in Definition 8.6 in terms of intertwining operators with Hermitian dual representations. Propositions 10.4 and 10.7 compute the Hermitian duals of standard quotient-type modules as standard sub-type modules. In the presence of (for example) a $c$-invariant form on $J(\Gamma)$, the conclusion is that

$$
I_{\mathrm{quo}}\left(\Gamma_{t}\right)^{h, \sigma_{c}} \simeq I_{\mathrm{sub}}\left(\Gamma_{t}\right) .
$$

The invariant pairings defining this isomorphism were constructed from a fixed isomorphism for the underlying spaces

$$
J: V(\Lambda)^{h} \simeq V(\Lambda) .
$$

Now it follows from the definitions that

$$
\left(\left[I_{\mathrm{quo}}(\Gamma)^{r}\right]^{h, \sigma_{c}}\right)^{\perp} \simeq I_{\mathrm{sub}}(\Gamma)^{-r+1},
$$

so that we get isomorphisms

$$
\left[I_{\text {quo }}(\Gamma)^{r} / I_{\text {quo }}(\Gamma)^{r+1}\right]^{h, \sigma_{c}} \simeq I_{\text {sub }}(\Gamma)^{-r} / I_{\text {sub }}(\Gamma)^{-r+1} .
$$

The isomorphism $J \circ L^{[r]}$ from $I_{\text {quo }}(\Gamma)^{r} / I_{\text {quo }}(\Gamma)^{r+1}$ to its Hermitian dual is the invariant Hermitian form in (4). 
Definition 14.9. Suppose $\Gamma$ is a Langlands parameter, and that the $\{$ def : jantzenform $\}$ Langlands quotient $J(\Gamma)$ admits a nonzero invariant or $c$-invariant Hermitian form $\langle$,$\rangle . Define nondegenerate invariant forms$

$$
\langle,\rangle^{[r]} \text { on } I_{\text {quo }}(\Gamma)^{r} / I_{\text {quo }}(\Gamma)^{r+1}
$$

as in Proposition 14.7. Write

$$
\left(\operatorname{pos}^{[r]}, \operatorname{neg}^{[r]}\right): \widehat{K} \rightarrow \mathbb{N} \times \mathbb{N}
$$

for the signatures of these forms (Proposition 8.9). The Jantzen form for $I_{\text {quo }}(\Gamma)$ is the nondegenerate form

$$
\sum_{r=0}^{\infty}\langle,\rangle^{[r]} \text { on gr } I_{\text {quo }}(\Gamma)==_{\text {def }} \sum_{r=0}^{\infty} I_{\text {quo }}(\Gamma)^{r} / I_{\text {quo }}(\Gamma)^{r+1} .
$$

Write

$$
\left(\operatorname{pos}_{I(\Gamma)}, \operatorname{neg}_{I(\Gamma)}\right)=\sum_{r=0}^{\infty}\left(\operatorname{pos}^{[r]}, \operatorname{neg}^{[r]}\right)
$$

for the signature of this form.

This is all that we need for the next few sections. Eventually, however, we will be studying these forms by deformation in $t$, and we will want to know how the signatures change with $t$.

Corollary 14.10 ([43], Theorem 3.8). Suppose $\Gamma$ is a Langlands parameter, and that the Langlands quotient $J(\Gamma)$ admits a nonzero invariant or c-invariant Hermitian form $\langle,\rangle_{1}$. Consider the family of standard representations $I_{\text {quo }}\left(\Gamma_{t}\right)$ (for $t \geq 0$ ) and the family of invariant forms $\langle,\rangle_{t}$ extending $\langle,\rangle_{1}$ as in Proposition 14.7. For every $t \geq 0$, define forms

$$
\langle,\rangle_{t}^{[r]} \text { on } I_{\mathrm{quo}}\left(\Gamma_{t}\right)^{r} / I_{\mathrm{quo}}\left(\Gamma_{t}\right)^{r+1}
$$

with signatures

$$
\left(\operatorname{pos}_{t}^{[r]}, \operatorname{neg}_{t}^{[r]}\right): \widehat{K} \rightarrow \mathbb{N} \times \mathbb{N}
$$

as in Definition 14.9. Write

$$
\left(\operatorname{pos}_{t}, \operatorname{neg}_{t}\right)=\sum_{r=0}^{\infty}\left(\operatorname{pos}_{t}^{[r]}, \operatorname{neg}_{t}^{[r]}\right)
$$

for the signature of the (nondegenerate) Jantzen form on gr $I_{\mathrm{quo}}\left(\Gamma_{t}\right)$. 
1. The subspace $I_{\mathrm{quo}}\left(\Gamma_{t}\right)^{1}=\operatorname{ker} L_{t}$ is zero unless $I_{\mathrm{quo}}\left(\Gamma_{t}\right)$ is reducible. This can happen only if either

a) there is a real root $\alpha$ such that

$$
\left\langle t \nu, \alpha^{\vee}\right\rangle \in \mathbb{Z} \backslash\{0\}, \quad \Lambda_{\mathfrak{q}}\left(m_{\alpha}\right)=(-1)^{\left\langle t \nu, \alpha^{\vee}\right\rangle+1}
$$

(notation as in $(9.3 \mathrm{~d}))$; or

b) there is a complex root $\delta$ such that $\left\langle(d \lambda, t \nu), \delta^{\vee}\right\rangle \in \mathbb{Z}$, and

$$
\left\langle t \nu, \delta^{\vee}\right\rangle>\left|\left\langle d \lambda, \delta^{\vee}\right\rangle\right|
$$

These conditions define a discrete set of values of $t$-in fact a subset of a lattice in $\mathbb{R}$. On the complement of this discrete set, the form $\langle,\rangle_{t}$ is nondegenerate and of locally constant signature

$$
\left(\operatorname{pos}_{t}, \operatorname{neg}_{t}\right)=\left(\operatorname{pos}_{t}^{[0]}, \operatorname{neg}_{t}^{[0]}\right) .
$$

2. Choose $\epsilon$ so small that $I\left(\Gamma_{t}\right)$ is irreducible for $t \in[1-\epsilon, 1)$ and for $t \in(1,1+\epsilon]$. Then

$$
\begin{gathered}
\left(\operatorname{pos}_{1+\epsilon}, \operatorname{neg}_{1+\epsilon}\right)=\left(\operatorname{pos}_{1}, \operatorname{neg}_{1}\right)=\sum_{r=0}^{\infty}\left(\operatorname{pos}_{1}^{[r]}, \operatorname{neg}_{1}^{[r]}\right) \\
\left(\operatorname{pos}_{1-\epsilon}, \operatorname{neg}_{1-\epsilon}\right)=\sum_{r \text { even }}\left(\operatorname{pos}_{1}^{[r]}, \operatorname{neg}_{1}^{[r]}\right)+\sum_{r^{\prime} \text { odd }}\left(\operatorname{neg}_{1}^{\left[r^{\prime}\right]}, \operatorname{pos}_{1}^{\left[r^{\prime}\right]}\right) .
\end{gathered}
$$

Equivalently,

$$
\begin{aligned}
& \operatorname{pos}_{1+\epsilon}=\operatorname{pos}_{1-\epsilon}+\sum_{r \text { odd }}\left(\operatorname{pos}_{1}^{[r]}-\operatorname{neg}_{1}^{[r]}\right), \\
& \operatorname{neg}_{1+\epsilon}=\operatorname{neg}_{1-\epsilon}+\sum_{r \text { odd }}\left(\operatorname{neg}_{1}^{[r]}-\operatorname{pos}_{1}^{[r]}\right) .
\end{aligned}
$$

That is, the signature of the form changes at reducible points according to the signature on the odd levels of the Jantzen filtration.

We will not reproduce the (elementary) proof from [43]. The main point is the limit formula at the end of Proposition 14.7; the factor $(s-1)^{-r}$ is positive unless $r$ is odd and $s<1$. 


\section{Signature characters for $c$-invariant forms}

In this section we will begin to explain what it means to "calculate" the signature of a $c$-invariant Hermitian form on an irreducible representation. We have already seen in Theorem 12.9 how to relate this to calculating the signature of a classical invariant Hermitian form. Just as in Section 14, the extension to ${ }^{\delta} G$ is relatively easy; so to keep the notation simple we discuss only $G$.

The idea is that the (Jantzen) Hermitian forms on standard modules (Definition 14.9) are taken as building blocks, and we seek to express the forms on irreducible modules in terms of these building blocks. In order to set the stage, we begin with the classical and simpler case of character theory.

Recall from (2.1c) the center of the enveloping algebra $\mathfrak{Z}(\mathfrak{g})$. Fix an \{se: charformulas\} algebra homomorphism ("infinitesimal character")

$$
\chi: \mathfrak{Z}(\mathfrak{g}) \rightarrow \mathbb{C}
$$

By Harish-Chandra's theorem, $\chi$ corresponds to a single Weyl group orbit $\mathcal{O}_{\mathfrak{h}}(\chi)$ in $\mathfrak{h}^{*}$, for every Cartan subalgebra $\mathfrak{h}$ in $\mathfrak{g}$. We will write

$$
\xi_{\lambda}: \mathfrak{Z}(\mathfrak{g}) \rightarrow \mathbb{C} \quad\left(\lambda \in \mathfrak{h}^{*}\right)
$$

for the homomorphism defined by $W \cdot \lambda$ (Theorem 5.4). Define

$$
\begin{gathered}
B(\chi)=\text { equivalence classes of Langlands parameters } \\
\text { of infinitesimal character } \chi
\end{gathered}
$$

(cf. Theorem 6.1). Sometimes it will be convenient to index the infinitesimal character by some particular weight $\lambda \in \mathfrak{h}^{*}$, and to write

$$
\begin{aligned}
B(\lambda) & =\text { classes of parameters of infinitesimal character } \xi_{\lambda} \\
& =B\left(\xi_{\lambda}\right) .
\end{aligned}
$$

We will occasionally need

$$
\begin{aligned}
B_{\text {weak }}(\lambda) & =\text { weak parameters of infinitesimal character } \xi_{\lambda} \\
& =B_{\text {weak }}\left(\xi_{\lambda}\right)
\end{aligned}
$$

(Definition 6.3).

In the notation of Theorem $6.1, \Gamma \in B(\chi)$ if and only if $d \gamma \in \mathcal{O}_{\mathfrak{h}}(\chi)$; equivalently, if and only if $\chi=\xi_{d \gamma}$. The set $B(\chi)$ is finite, of cardinality bounded by the sum over conjugacy classes of real Cartan subgroups $H$ of

$$
\left[H: H_{0}\right][W(\mathfrak{g}, \mathfrak{h}): W(G, H)] .
$$


Define

$$
\begin{aligned}
m_{\Xi, \Gamma} & =(\text { multiplicity of } J(\Xi) \text { as composition factor of } I(\Gamma)) \\
& ={ }_{\text {def }} \quad m_{I(\Gamma)}(J(\Xi)) \in \mathbb{N} .
\end{aligned}
$$

$\{$ eq: multformstd $\}$

In the Grothendieck group of virtual $\left(\mathfrak{g}_{0}, K\right)$-modules, this is the same as

$$
[I(\Gamma)]=\sum_{\Xi} m_{\Xi, \Gamma}[J(\Xi)]
$$

(The brackets denote the image in the Grothendieck group, and serve as a reminder that this equality need not be true on the level of modules.) Because the center of the enveloping algebra acts by scalars on the standard module $I(\Gamma)$, the multiplicity $m_{\Xi, \Gamma}$ can be nonzero only if $\Xi$ and $\Gamma$ belong to the same set $B(\chi)$. So we can regard $m$ as a finite matrix of nonnegative integers, with entries indexed by the finite set $B(\chi)$. Theorem 6.1 says that the diagonal entries are equal to one:

$$
m_{\Gamma, \Gamma}=1
$$

We can impose a preorder $\leq_{\mathrm{RP}}$ on $B(\chi)$ by the length of the real part of the continuous parameter. (In Definition 18.5 below, we will introduce the Bruhat order, which is a partial order on $B(\chi)$ with fewer relations than this one, but still satisfying the analogue of (15.1i). The point of using $\leq_{R P}$ is simply to get quickly a weak version of upper triangularity, so that we know certain matrices are invertible.) Langlands' proof of Theorem 6.1 shows that

$$
m_{\Xi, \Gamma} \neq 0 \Longrightarrow \Xi \leq_{\mathrm{RP}} \Gamma
$$

\{eq:uppertri\}

and that if "equality holds" (that is, if the real parts of the continuous parameters have the same length) then $\Xi=\Gamma$. Therefore the matrix $m$ is upper triangular with integer entries and ones on the diagonal. Accordingly it has an inverse with these same properties: we can define $M_{\Gamma, \Psi} \in \mathbb{Z}$ by the requirement

$$
\sum_{\Gamma} m_{\Xi, \Gamma} M_{\Gamma, \Psi}=\delta_{\Xi, \Psi} \quad(\Xi, \Psi, \Gamma \in B(\chi))
$$

This is equivalent to an equation in the Grothendieck group of virtual $\left(\mathfrak{g}_{0}, K\right)$-modules,

$$
[J(\Psi])=\sum_{\Gamma} M_{\Gamma, \Psi}[I(\Gamma)] .
$$

$\{$ eq:repformirr $\}$ 
We can also write this as an equation for distribution characters (Theorem $5.12)$ :

$$
\Theta_{J(\Psi)}=\sum_{\Gamma} M_{\Gamma, \Psi} \Theta_{I(\Gamma)}
$$

Since the characters of the standard modules $I(\Gamma)$ were (in principle) computed by Harish-Chandra, this formula expresses the character of an irreducible representation $J(\Xi)$ in terms of known distributions $\Theta_{I(\Gamma)}$, and a matrix of integers $M_{\Gamma, \Psi}$. The integers $M_{\Gamma, \Psi}$ are computed by the KazhdanLusztig conjectures (to which we will return in Sections 18 and 19).

All of this discussion takes place in the Grothendieck group of finite length $\left(\mathfrak{g}_{0}, K\right)$-modules. To make a parallel discussion of invariant forms, we need something like a "Grothendieck group of modules with a nondegenerate invariant form." The difficulty with this idea is that the restriction of a nondegenerate form to a submodule may be degenerate; so the category of modules with a nondegenerate form is not abelian, and it is not so clear how to define a Grothendieck group. This issue is addressed by Lemma 3.9 in [43]; we repeat the argument (phrasing it a little more formally and generally) in the next few results, culminating in Proposition 15.11.

Lemma 15.2. In the setting of Proposition 8.9, suppose $M$ is an admissible $(\mathfrak{h}, L)$ module of finite length, admitting a nondegenerate $\sigma$-invariant form $\langle,\rangle_{M}$.

1. The form $\langle,\rangle_{M}$ defines an isomorphism $M \simeq M^{h, \sigma}$ (Definition 8.6).

2. Forming orthogonal complement

$$
N^{\perp}={ }_{\operatorname{def}}\{m \in M \mid\langle m, n\rangle=0 \text {, all } n \in N\}
$$

is inclusion-reversing on submodules of $M$, and $\left(N^{\perp}\right)^{\perp}=N$.

3. The isomorphism of (1) restricts to

$$
N^{h, \sigma} \simeq M / N^{\perp} .
$$

4. The radical of the invariant form

$$
\langle,\rangle_{N}={ }_{\text {def }} \text { restriction of }\langle,\rangle_{M} \text { to } N
$$

is equal to $N \cap N^{\perp}$.

5. If $N_{1}$ and $N_{2}$ are submodules, then

$$
\left(N_{1} \cap N_{2}\right)^{\perp}=N_{1}^{\perp}+N_{2}^{\perp}, \quad\left(N_{1}+N_{2}\right)^{\perp}=N_{1}^{\perp} \cap N_{2}^{\perp} .
$$


This is entirely elementary; the admissibility hypothesis allows us to focus on the finite-dimensional multiplicity spaces $M^{\delta}$ defined in (8.8).

Proposition 15.3. In the setting of Proposition 8.9, suppose $M$ is an admissible $(\mathfrak{h}, L)$ module of finite length, with a nondegenerate $\sigma$-invariant form $\langle,\rangle_{M}$. Suppose $S$ is an $(\mathfrak{h}, L)$-submodule of $M$. Use the notation of Lemma 15.2. Define

$$
R=S \cap S^{\perp}=\text { radical of form restricted to } S .
$$

1. The form $\langle,\rangle_{S+S^{\perp}}$ has radical equal to $R$, and so descends to a nondegenerate form on $\left(S+S^{\perp}\right) / R$.

2. There is an orthogonal direct sum decomposition

$$
\left(S+S^{\perp}\right) / R=(S / R) \oplus\left(S^{\perp} / R\right) .
$$

3. The form $\langle,\rangle_{M}$ provides a natural identification

$$
R^{h, \sigma} \simeq M / R^{\perp}=M /\left(S+S^{\perp}\right)
$$

4. The signature

$$
\left(\operatorname{pos}_{M}, \operatorname{neg}_{M}\right): \widehat{K} \rightarrow \mathbb{N} \times \mathbb{N}
$$

is given by

$$
\begin{aligned}
\operatorname{pos}_{M} & =\operatorname{pos}_{S / R}+\operatorname{pos}_{S^{\perp} / R}+\operatorname{mult}_{R}, \\
\operatorname{neg}_{M} & =\operatorname{neg}_{S / R}+\operatorname{neg}_{S^{\perp} / R}+\text { mult }_{R} .
\end{aligned}
$$

Here mult $_{R}$ is the multiplicity function for the module $R$.

The proof is immediate from the lemma.

If $A$ is any admissible $(\mathfrak{h}, L)$-module, then the direct sum $A \oplus A^{h, \sigma}$ carries a natural nondegenerate form vanishing on both summands; the formula is

$$
\left\langle(a, \xi),\left(a^{\prime}, \xi^{\prime}\right)\right\rangle_{\mathrm{hyp}}=\xi\left(a^{\prime}\right)+\overline{\xi^{\prime}(a)} .
$$

This is called the hyperbolic form. Its signature is easily computed to be

$$
\operatorname{pos}_{A \oplus A^{h, \sigma}}=\operatorname{mult}_{A}, \quad \operatorname{neg}_{A \oplus A^{h, \sigma}}=\operatorname{mult}_{A} .
$$

The proposition says that, in terms of signatures of invariant forms, the module $M$ looks like

$$
\left(R \oplus R^{h, \sigma}\right) \oplus(S / R) \oplus\left(S^{\perp} / R\right) .
$$


It need not be the case that $M$ has this decomposition as a module. For us such modules will arise from "wall-crossing" translation functors. For example, there is a representation of $S L(2, \mathbb{R})$ carrying a nondegenerate invariant Hermitian form and three irreducible composition factors: a discrete series representation with $S O(2)$-types indexed by $\{2,4,6, \ldots\}$, appearing twice, and the trivial representation (having only the $S O(2)$-type indexed by zero). The form has signature $(1,1)$ on each of the nontrivial $S O(2)$-types. There is a submodule $S$ containing the trivial representation and one copy of the discrete series. On this submodule the radical $R$ is the discrete series representation, $S^{\perp}$ is equal to $R$, and $S+S^{\perp}=S$.

Definition 15.6. Suppose (as in Proposition 8.9) that $(\mathfrak{h}, L(\mathbb{C})$ ) is a pair with $L(\mathbb{C})$ reductive, and that $\sigma$ is a real structure defining a compact form $L$ (Definition 8.1). The Grothendieck group of finite length admissible $(\mathfrak{h}, L(\mathbb{C}))$-modules with nondegenerate $\sigma$-invariant Hermitian forms (briefly, the Hermitian Grothendieck group) is the abelian group $\mathcal{G}(\mathfrak{h}, L(\mathbb{C}))^{\sigma}$ generated by such $\left(M,\langle,\rangle_{M}\right)$, subject to the following relations. Write $\left[M,\langle,\rangle_{M}\right]$ for the class in the Grothendieck group. Whenever $S$ is a submodule of $M$, and $R$ is the radical of the restricted form $\langle,\rangle_{S}$, then we impose the relation

$$
\left[M,\langle,\rangle_{M}\right]=\left[R \oplus R^{h, \sigma},\langle,\rangle_{\mathrm{hyp}}\right]+\left[S / R,\langle,\rangle_{S / R}\right]+\left[S^{\perp} / R,\langle,\rangle_{S^{\perp} / R}\right] .
$$

The motivation for the definition is Proposition 15.3. That proposition provides exact sequences

$$
\begin{gathered}
0 \rightarrow R^{\perp} \rightarrow M \rightarrow R^{h, \sigma} \rightarrow 0 \\
0 \rightarrow R \rightarrow R^{\perp} \rightarrow S / R \oplus S^{\perp} / R \rightarrow 0,
\end{gathered}
$$

and therefore an equality

$$
[M]=\left[R \oplus R^{h, \sigma}\right]+[S / R]+\left[S^{\perp} / R\right]
$$

in the ordinary Grothendieck group $\mathcal{G}(\mathfrak{h}, L(\mathbb{C})$ ) of admissible finite-length $(\mathfrak{h}, L(\mathbb{C}))$-modules. From this we conclude that there is a natural homomorphism of abelian groups

$$
\mathcal{G}(\mathfrak{h}, L(\mathbb{C}))^{\sigma} \rightarrow \mathcal{G}(\mathfrak{h}, L(\mathbb{C})), \quad\left[M,\langle,\rangle_{M}\right] \mapsto[M]
$$

defined by forgetting the form. Proposition 15.3 also implies that there is a well-defined signature homomorphism

$$
\begin{aligned}
\left(\operatorname{pos}_{\bullet}, \operatorname{neg}_{\bullet}\right): \mathcal{G}(\mathfrak{h}, L(\mathbb{C}))^{\sigma} & \rightarrow \operatorname{Map}(\widehat{L}, \mathbb{Z} \times \mathbb{Z}), \\
{\left[M,\langle,\rangle_{M}\right] } & \mapsto\left(\operatorname{pos}_{M}, \operatorname{neg}_{M}\right) .
\end{aligned}
$$

The fundamental fact about the ordinary Grothendieck group is 
Proposition 15.8. Suppose that $(\mathfrak{h}, L(\mathbb{C}))$ is a pair with $L(\mathbb{C})$ reductive \{prop:grothgens\} (Definition 8.1). The Grothendieck group of admissible $(\mathfrak{h}, L(\mathbb{C})$ )-modules of finite length is a free abelian group (that is, a free $\mathbb{Z}$-module) with generators the (equivalence classes of) irreducible admissible $(\mathfrak{h}, L(\mathbb{C}))$-modules. There is a well-defined multiplicity homomorphism

$$
\text { mult. }_{\bullet} \mathcal{G}(\mathfrak{h}, L(\mathbb{C})) \rightarrow \operatorname{Map}(\widehat{L}, \mathbb{Z}), \quad[M] \mapsto\left(\operatorname{mult}_{M}\right) .
$$

We want a corresponding statement about the Hermitian Grothendieck group. In the preceding proposition, the ring of ordinary integers appears as the Grothendieck group of complex vector spaces. Roughly speaking, the integers appearing are dimensions of "multiplicity spaces" $\operatorname{Hom}_{\mathfrak{h}, L(\mathbb{C})}(J, M)$, with $J$ an irreducible module. (Of course this statement is not precisely correct: the indicated Hom is too small to capture the full multiplicity of $J$ in $M$.) For the Hermitian case, the role of $\mathbb{Z}$ is played by the Grothendieck group of vector spaces with nondegenerate forms.

Definition 15.9. The signature ring is the Hermitian Grothendieck group $\mathbb{W}$ of finite-dimensional vector spaces with nondegenerate Hermitian forms. (This is the Hermitian Grothendieck group

$$
\mathbb{W}=\mathcal{G}(0,\{1\})^{\sigma}
$$

of Definition 15.6 in the case when the Lie algebra $\mathfrak{h}$ is zero and the group $L(\mathbb{C})$ is trivial.) The ring structure is defined by tensor product of Hermitian forms. The ordinary Grothendieck group of this category is $\mathbb{Z}$, so we get as in (15.7) an algebra homomorphism

$$
\text { for }: \mathbb{W} \rightarrow \mathbb{Z}
$$

by forgetting the form.

The identity element of $\mathbb{W}$ is the class of the one-dimensional space $\mathbb{C}$ with its standard (positive) Hermitian form $\langle z, w\rangle=z \bar{w}$ :

$$
1=[\mathbb{C},\langle,\rangle]
$$

We also use the element

$$
s=[\mathbb{C},-\langle,\rangle] .
$$

Taking the tensor square of this form eliminates the minus sign, so

$$
s^{2}=1
$$


Sylvester's law of inertia for Hermitian forms implies that any finitedimensional vector space $V$ with a nondegenerate form $\langle,\rangle_{V}$ is isomorphic to a sum of copies of these two cases:

$$
\left[V,\langle,\rangle_{V}\right]=p \cdot 1+q \cdot s, \quad(p, q \in \mathbb{N})
$$

Furthermore

$$
\begin{aligned}
\mathbb{W} & =\{p \cdot 1+q \cdot s \mid p, q \in \mathbb{Z}\} \\
& \simeq \mathbb{Z}[s] /\left\langle s^{2}-1\right\rangle .
\end{aligned}
$$

The reduction map (forgetting the form) is

$$
\text { for }: \mathbb{W} \rightarrow \mathbb{Z}, \quad \text { for }(p+q s)=p+q .
$$

The ring $\mathbb{W}$ allows us to make a small consolidation of notation. In the setting of Proposition 8.9, suppose $\left(V,\langle,\rangle_{V}\right)$ is an admissible $(\mathfrak{h}, L(\mathbb{C}))$ module with a $\sigma$-invariant Hermitian form. We can define the signature character of $V$ to be the function

$$
\begin{gathered}
\operatorname{sig}_{V}: \widehat{K} \rightarrow \mathbb{W}, \\
\operatorname{sig}_{V}(\delta)=\left[V^{\delta} /(\text { radical of form }),\langle,\rangle_{V}^{\delta}\right]=\operatorname{pos}_{V}(\delta)+\operatorname{neg}_{V}(\delta) s
\end{gathered}
$$

(notation as in Proposition 8.9(4).)

Proposition 15.11. In the setting of Definition 15.6 and Definition 15.9, forming the tensor product of vector spaces with $(\mathfrak{h}, L(\mathbb{C})$ )-modules (each endowed with a nondegenerate Hermitian form) defines the structure of a $\mathbb{W}$-module on the Hermitian Grothendieck group $\mathcal{G}(\mathfrak{h}, L(\mathbb{C}))^{\sigma}$.

1. This Grothendieck group has the following set of generators as a $\mathbb{W}$ module:

(a) For each irreducible admissible $(\mathfrak{h}, L(\mathbb{C}))$-module $J$ such that $J \simeq$ $J^{h, \sigma}$, fix a "base" choice of nondegenerate $\sigma$-invariant form $\langle,\rangle_{J, b}$. Then the generator is $\left[J,\langle,\rangle_{J, b}\right]$; the subscript b stands for "base."

(b) For each inequivalent (unordered) pair of irreducible admissible $(\mathfrak{h}, L(\mathbb{C}))$-modules $J^{\prime}$ and $J^{\prime h, \sigma}$, the generator is $\left[J^{\prime} \oplus J^{\prime h, \sigma},\langle,\rangle_{\text {hyp }}\right]$ (cf. (15.4)). This generator satisfies the relation

$$
s \cdot\left[J^{\prime} \oplus J^{\prime h, \sigma},\langle,\rangle_{h y p}\right]=\left[J^{\prime} \oplus J^{\prime h, \sigma},\langle,\rangle_{h y p}\right] .
$$

2. The Hermitian Grothendieck group is a free $\mathbb{W}$-module over these generators, except for the relations indicated in 1(b). 
3. Any admissible $(\mathfrak{h}, L(\mathbb{C}))$-module $M$ with a nondegenerate $\sigma$-invariant form $\langle,\rangle_{M}$ may be written uniquely as

$$
\begin{aligned}
{\left[M,\langle,\rangle_{M}\right]=} & \sum_{J \simeq J^{h, \sigma}}\left(p_{J}(M)+q_{J}(M) s\right)\left[J,\langle,\rangle_{J, b}\right] \\
& +\sum_{J^{\prime} \nsucceq J^{\prime h, \sigma}} m_{J^{\prime}}(M)\left[J^{\prime} \oplus J^{\prime h, \sigma},\langle,\rangle_{h y p}\right] \\
= & \sum_{J \simeq J^{h, \sigma}} w_{J}(M)\left[J,\langle,\rangle_{J, b}\right] \\
& +\sum_{J^{\prime} \nsucceq J^{\prime h, \sigma}} m_{J^{\prime}}(M)\left[J^{\prime} \oplus J^{\prime h, \sigma},\langle,\rangle_{h y p}\right] .
\end{aligned}
$$

Here all the $p_{J}(M), q_{J}(M)$, and $m_{J^{\prime}}(M)$ are nonnegative integers; $m_{J^{\prime}}(M)$ is the multiplicity of $J^{\prime}$ as a composition factor of $M$; and

$$
p_{J}(M)+q_{J}(M)=m_{J}(M)
$$

the multiplicity of $J$ as a composition factor of $M$. The elements

$$
w_{J}(M)={ }_{\operatorname{def}} p_{J}(M)+q_{J}(M) s
$$

are in $\mathbb{W}$; in the formula $m_{J^{\prime}}(M)$ could be replaced by any element $w^{\prime}$ of $\mathbb{W}$ with for $\left(w^{\prime}\right)=m_{J^{\prime}}(M)$.

4. The signature character of $M$ may be computed from those of the irreducible composition factors and the coefficients in (3):

$$
\operatorname{sig}_{M}=\sum_{J \simeq J^{h, \sigma}} w_{J}(M) \operatorname{sig}_{J, b}+\sum_{J^{\prime} \nsucceq J^{\prime h, \sigma}} m_{J^{\prime}}(M) \operatorname{mult}_{J^{\prime}}(1+s) .
$$

Proof. We begin by proving the existence of the indicated decomposition of $M$, by induction on the length (the number of irreducible composition factors) of $M$. If the length is zero, then $M=0$, and we can take all the coefficients $p_{J}(M), q_{J}(M)$, and $m_{J^{\prime}}(M)$ to be zero. So suppose that the length is $\ell>0$, and that the decomposition is available for modules of length at most $\ell-1$. Since $M$ has positive (finite) length, it admits an irreducible submodule $S$. Now there are two cases.

Case 1: $\langle,\rangle_{S}=0$. In the notation of Proposition 15.3, this means that $S=R \subset S^{\perp}$, and that $M / S^{\perp} \simeq R^{h, \sigma}$. The module

$$
M_{0}=S^{\perp} / R
$$


inherits a nondegenerate form $\langle,\rangle_{M_{0}}$, and has length $\ell-2$ (since the two irreducible composition factors $R$ and $R^{h, \sigma}$ have been removed). By Definition 15.6, we have

$$
\left[M,\langle,\rangle_{M}\right]=\left[R \oplus R^{h, \sigma},\langle,\rangle_{\mathrm{hyp}}\right]+\left[M_{0},\langle,\rangle_{M_{0}}\right]
$$

By inductive hypothesis, $M_{0}$ has an expression of the desired form.

If $R \not R^{h, \sigma}$, then $\left(R, R^{h, \sigma}\right)$ is one of the pairs in the second sum. So we get the expression for $M$ just by adding 1 to the coefficient $m_{R}$ in that sum.

If $R \simeq R^{h, \sigma}$, then $R$ is one of the $J$ in the first sum. It is an elementary exercise that

$$
\left(R \oplus R^{h, \sigma},\langle,\rangle_{\text {hyp }}\right) \simeq\left(R,\langle,\rangle_{R}\right) \oplus\left(R,-\langle,\rangle_{R}\right)
$$

So we get the expression for $M$ by adding $1+s$ to the coefficient of $R$ in the first sum.

Case 2: $\langle,\rangle_{S} \neq 0$. Because $S$ was chosen irreducible, this means that

$$
\left(S,\langle,\rangle_{S}\right) \simeq\left(S, \pm\langle,\rangle_{S, b}\right)
$$

In particular, this form is nondegenerate. In the notation of Proposition 15.3 , this means that $R=0$, and

$$
\left(M,\langle,\rangle_{M}\right)=\left(S,\langle,\rangle_{S}\right) \oplus\left(S^{\perp},\langle,\rangle_{S^{\perp}}\right)
$$

By Definition 15.6, the corresponding relation is true also in the Hermitian Grothendieck group. Now the module $M_{0}=S^{\perp}$ has length $\ell-1$ (since the irreducible composition factor $S$ has been removed). By inductive hypothesis, $M_{0}$ has an expression of the desired form. We get the expression for $M$ by adding 1 to $p_{S}\left(M_{0}\right)$ (in the case of a plus sign) or to $q_{S}\left(M_{0}\right)$ (in the case of a minus sign). This completes the inductive proof of the existence of the expression for $M$; the proof also shows that the coefficients are nonnegative integers, with the indicated relationship to composition series multiplicity.

The existence of these expressions proves that the indicated elements generate the Hermitian Grothendieck group.

The statement about signature characters in (4) is a consequence of Proposition 15.3(4) and the definition of $\mathbb{W}$. We will make no use of the statement about (the absence of) relations in the Hermitian Grothendieck group, so we omit the proof.

Using the description of the Hermitian Grothendieck group in Proposi\{se: cinvtsigformulas\} tion 15.11, we can extend the discussion in (15.1) to invariant forms. Partly 
to avoid small technical difficulties (like the possible nonexistence of invariant forms), and partly because it is what we need first, we will consider here a real infinitesimal character ([38], Definition 5.4.11)

$$
\chi: \mathfrak{Z}(\mathfrak{g}) \rightarrow \mathbb{C} .
$$

This means that the parameter set

$$
B(\chi)=\text { Langlands parameters of infinitesimal character } \chi
$$

consists of parameters with real continuous part (see the discussion after Theorem 7.1). We take the real structure $\sigma_{c}$ as in (10.1), and consider the Hermitian Grothendieck group

$$
\mathcal{G}(\mathfrak{g}, K)^{c}
$$

of finite-length $(\mathfrak{g}, K)$ modules with nondegenerate $c$-invariant Hermitian forms. According to Proposition 10.7, for each $\Gamma \in B(\chi)$ there is a canonical nonzero $c$-invariant form

$$
\langle,\rangle_{J(\Gamma), b}^{c}
$$

characterized (up to a positive multiple) by being positive on every lowest $K$-type. According to Proposition 15.11, the elements

$$
\left[J(\Gamma),\langle,\rangle_{J(\Gamma), b}^{c}\right]
$$

constitute a basis for (the infinitesimal character $\chi$ part of $) \mathcal{G}(\mathfrak{g}, K)^{c}$.

Definition 14.9 explains how to pass from $\langle,\rangle_{J(\Gamma), b}^{c}$ to a nondegenerate (Jantzen) form

$$
\langle,\rangle_{I(\Gamma)}^{c} \text { on } \operatorname{gr} I_{\text {quo }}(\Gamma) .
$$

Then Proposition 15.11 allows us to write this Jantzen form in the Hermitian Grothendieck group as a $\mathbb{W}$-linear combination of the basis of irreducibles:

$$
\left[I(\Gamma),\langle,\rangle_{I(\Gamma)}^{c}\right]=\sum_{\Xi} w_{\Xi, \Gamma}^{c}\left[J(\Xi),\langle,\rangle_{J(\Xi), b}^{c}\right] .
$$

$\{$ eq: formf ormstd $\}$

Each coefficient

$$
w_{\Xi, \Gamma}^{c}=p_{\Xi, \Gamma}^{c}+q_{\Xi, \Gamma}^{c} s \in \mathbb{W}
$$

has nonnegative $p^{c}$ and $q^{c}$, and

$$
\operatorname{for}\left(w_{\Xi, \Gamma}^{c}\right)=_{\operatorname{def}} p_{\Xi, \Gamma}^{c}+q_{\Xi, \Gamma}^{c}=m_{\Xi, \Gamma} .
$$


These facts imply that

$$
w_{\Xi, \Gamma}^{c} \neq 0 \text { if and only if } m_{\Xi, \Gamma} \neq 0 .
$$

\{eq:multboundssig\}

It follows that, just as for the multiplicity matrix, the coefficient $w_{\Xi, \Gamma}^{c}$ can be nonzero only if $\Xi$ and $\Gamma$ belong to the same set $B(\chi)$. So we can regard $w^{c}$ as a finite matrix of elements of the commutative ring $\mathbb{W}$, with entries indexed by the finite set $B(\chi)$. Proposition $14.7(2)$ says that the diagonal entries are equal to one:

$$
w_{\Gamma, \Gamma}^{c}=1 .
$$

The fact that the multiplicity matrix is upper triangular ((15.1i), together with (15.12j), imply

$$
w_{\Xi, \Gamma}^{c} \neq 0 \Longrightarrow \Xi \leq_{\mathrm{RP}} \Gamma
$$

and that if "equality holds" (that is, if the real parts of the continuous parameters have the same length) then $\Xi=\Gamma$. Therefore the matrix $w^{c}$ is upper triangular with entries in $\mathbb{W}$ and ones on the diagonal. Accordingly it has an inverse with these same properties: we can define $W_{\Gamma, \Psi}^{c} \in \mathbb{W}$ by the requirement

$$
\sum_{\Gamma} w_{\Xi, \Gamma}^{c} W_{\Gamma, \Psi}^{c}=\delta_{\Xi, \Psi} \quad(\Xi, \Psi, \Gamma \in B(\chi)) .
$$

This is equivalent to an equation in the Hermitian Grothendieck group of virtual $\left(\mathfrak{g}_{0}, K\right)$-modules,

$$
\left[J(\Psi),\langle,\rangle_{J(\Psi), b}^{c}\right]=\sum_{\Gamma} W_{\Gamma, \Psi}^{c}\left[I(\Gamma),\langle,\rangle_{I(\Gamma)}^{c}\right] .
$$

\{eq:formformirr\}

We can also write this as an equation for signature functions (see (15.10))

$$
\operatorname{sig}_{J(\Psi)}^{c}=\sum_{\Gamma} W_{\Gamma, \Psi}^{c} \operatorname{sig}_{I(\Gamma)}^{c}
$$

\{eq:sigformirr $\}$

Formally this equation appears to calculate signatures (for $c$-invariant forms) for the irreducible modules $J(\Psi)$ in terms of the finite matrix $W^{c}$, each entry of which is a pair of integers. This sounds very good for the problem of computing signatures explicitly. Nevertheless this formula is less satisfactory than (15.1l) for two reasons. First, there is no Kazhdan-Lusztig conjecture to compute the coefficient matrix $W_{\Gamma, \Psi}^{c}$. We will address this in Section 20. Second, we do not understand so well the signature functions $\operatorname{sig}_{I(\Gamma)}^{c}$ for the standard modules $I(\Gamma)$. We will address this question in Section 21.

We conclude this section by rewriting Corollary 14.10 in our attractive new notation. 
Corollary 15.13 ([43], Theorem 3.8). Suppose $\Gamma$ is a Langlands parameter, and that the Langlands quotient $J(\Gamma)$ admits a nonzero invariant or c-invariant Hermitian form $\langle,\rangle_{1}$. Consider the family of standard representations $I_{\mathrm{quo}}\left(\Gamma_{t}\right)$ (for $t \geq 0$ ) defined in (14.1), and the family of invariant or c-invariant forms $\langle,\rangle_{t}$ extending $\langle,\rangle_{1}$ as in Proposition 14.7. For every $t \geq 0$, define forms

$$
\langle,\rangle_{t}^{[r]} \text { on } I_{\mathrm{quo}}\left(\Gamma_{t}\right)^{r} / I_{\mathrm{quo}}\left(\Gamma_{t}\right)^{r+1}
$$

with signatures

$$
\left.\operatorname{sig}_{I\left(\Gamma_{t}\right)}^{[r]}=\operatorname{pos}_{t}^{[r]}+s \operatorname{neg}_{t}^{[r]}\right): \widehat{K} \rightarrow \mathbb{W}
$$

as in Definition 15.9. Write

$$
\operatorname{sig}_{t}=\operatorname{sig}_{I\left(\Gamma_{t}\right)}=\sum_{r=0}^{\infty} \operatorname{sig}_{I\left(\Gamma_{t}\right)}^{[r]}
$$

for the signature of the (nondegenerate) Jantzen form on gr $I_{\mathrm{quo}}\left(\Gamma_{t}\right)$. Consider a finite subset

$$
0<t_{r}<t_{r-1}<\cdots<t_{1} \leq 1 \subset(0,1] .
$$

so that $I\left(\Gamma_{t}\right)$ is irreducible for $t \in(0,1] \backslash\left\{t_{i}\right\}$.

1. On the complement of $\left\{t_{i}\right\}$, the form $\langle,\rangle_{t}$ is nondegenerate and of locally constant signature

$$
\operatorname{sig}_{t}=\operatorname{sig}_{t}^{[0]}
$$

2. In terms of the "signature matrix" defined in (15.12g),

$$
\operatorname{sig}_{1}=\sum_{\Xi \in B(\chi)} w_{\Xi, \Gamma}^{c} \operatorname{sig}_{J(\Xi)}
$$

3. Choose $\epsilon$ so small that $I\left(\Gamma_{t}\right)$ is irreducible for $t \in[1-\epsilon, 1)$ and for $t \in(1,1+\epsilon]$. Then

$$
\begin{gathered}
\operatorname{sig}_{1+\epsilon}=\operatorname{sig}_{1}=\sum_{r=0}^{\infty} \operatorname{sig}_{1}^{[r]}, \\
\operatorname{sig}_{1-\epsilon}=\sum_{r \text { even }} \operatorname{sig}_{1}^{[r]}+\sum_{r^{\prime} \text { odd }} s \cdot \operatorname{sig}_{1}^{\left[r^{\prime}\right]} .
\end{gathered}
$$


Equivalently,

$$
\operatorname{sig}_{1+\epsilon}=\operatorname{sig}_{1-\epsilon}+(s-1) \sum_{r \text { odd }} \operatorname{sig}_{1}^{[r]} .
$$

That is, the signature of the form changes at reducible points according to the signature on the odd levels of the Jantzen filtration.

\section{Translation functors: first facts}

Our next serious goal is to introduce the Kazhdan-Lusztig " $q$-analogues" of the multiplicities and signatures defined in Section 15. The powers of $q$ appearing will come from a grading on the set $B(\chi)$ of Langlands parameters with a fixed infinitesimal character (see (15.1)). This grading is combinatorially understandable only in the case of regular infinitesimal character $\chi$. We will define it for singular $\chi$ by some kind of "collapsing" from the regular case, which makes sense because of the Jantzen-Zuckerman translation principle. Our purpose in this section is to say enough about the translation functors to explain these definitions.

Translation functors are defined using finite-dimensional representations of $G$, and in particular by thoroughly understanding their weights. These matters extend to ${ }^{\delta} G$ only with care and effort. We will therefore present the theory for $G$ in this section, and discuss the extension to ${ }^{\delta} G$ in Section 17.

Recall from (3.1) that $G$ is assumed to be the group of real points of a connected complex reductive algebraic group $G(\mathbb{C})$. So far we have made serious use of only two aspects of this hypothesis. The first is that $G$ is in the Harish-Chandra class, meaning that the automorphism $\operatorname{Ad}(g)$ of the complexified Lie algebra $\mathfrak{g}$ is inner. This requirement means that $\operatorname{Ad}(G)$ acts trivially on the center $\mathfrak{Z}(\mathfrak{g})$ of $U(\mathfrak{g})$, which in turn implies that $\mathfrak{Z}(\mathfrak{g})$ must act by scalars on any irreducible $(\mathfrak{g}, K)$-module.

The second aspect is that the Cartan subgroups of $G$ (the centralizers in $G$ of Cartan subalgebras of $\mathfrak{g}$ that are defined over $\mathbb{R}$ ) are all abelian. One effect of this is to simplify slightly the statement of the Langlands classification, because the character (of a double cover of a Cartan subgroup) appearing in a Langlands parameter is necessarily one-dimensional. A more subtle consequence is that the lowest $K$-types have multiplicity one (Proposition 6.6).

A third aspect of our category of groups is that $G$ is isomorphic to a group of matrices. So far we have not made serious use of this hypothesis. In the theory of translation functors it is necessary for the simple formulation below 
of Theorem 16.6(2) (the behavior of Langlands parameters under translation functors).

Even to formulate a theory of translation functors we will make use of the first two properties again, through the following two propositions.

Proposition 16.1. Suppose $G$ is a real reductive group as in (3.1). Fix \{prop:findimlwts\} a real Cartan subgroup $H$ of $G$, and a set $R^{+} \subset R(G, H)$ of positive roots (Definition 5.2).

1. Suppose that $F$ is a finite-dimensional irreducible representation of $G$. Then the $R^{+}$-highest weight space of $F$ is a one-dimensional irreducible representation $\phi=\phi(F) \in \widehat{H}$.

2. Write $d \psi \in \mathfrak{h}^{*}$ for the differential of any (one-dimensional) character $\psi$ of $H_{s}$. The set

$$
\Delta(F, H) \subset \widehat{H}
$$

of weights of the finite-dimensional irreducible representation $F$ is (if we write $\langle S\rangle$ for the convex hull of a subset $S$ of a vector space)

$$
\Delta(F, H)=\{\psi \in \widehat{H} \mid \phi-\psi \in \mathbb{Z} R(G, H), \quad d \psi \in\langle W(\mathfrak{g}, \mathfrak{h}) \cdot d \phi\rangle\} .
$$

Define $\Lambda_{\text {fin }}(G, H) \subset \widehat{H}$ to be the group of weights of finite-dimensional representations of $G$.

3. The dominant weights in $\Lambda_{f i n}(G, H)$ are the highest weights of finitedimensional representations of $G$.

4. A weight $\psi \in \widehat{H}$ belongs to $\Lambda_{f i n}(G, H)$ if and only we have both

a) $d \psi\left(\beta^{\vee}\right) \in \mathbb{Z}$ for every coroot $\beta^{\vee} \in R^{\vee}(\mathfrak{g}, \mathfrak{h})$, and

b) $\psi\left(m_{\alpha}\right)=(-1)^{\psi\left(\alpha^{\vee}\right)}$ for every real coroot $\alpha^{\vee}$ (Definition 5.7).

Proposition 16.2. Suppose $G$ is a real reductive group as in (3.1). Fix a \{prop:findimlHswts maximally split real Cartan subgroup $H_{s}$ of $G$, preserved by the Cartan involution $\theta$. Write $H_{s}=T_{s} A_{s}$ for the Cartan decomposition of $H_{s}$ (Proposition 4.3). That $H_{s}$ is maximally split is equivalent to either of the conditions

a) $\mathfrak{a}_{s, 0}$ is a maximal abelian subalgebra of the -1 eigenspace $\mathfrak{s}_{0}$ of $\theta$; or

b) every imaginary root of $\mathfrak{h}_{s}$ in $\mathfrak{g}$ is compact (Definition 5.7).

Fix a set of positive roots $R_{s}^{+}$for $R\left(\mathfrak{g}, \mathfrak{h}_{s}\right)$. 
1. The group $H_{s}$ meets every component of $G$ : i.e., $G=G_{0} \cdot H_{s}$.

2. Suppose $F$ is a finite-dimensional irreducible representation of $G$, and $\phi_{s}=\phi_{s}(F) \in \widehat{H_{s}}$ is the $R_{s}^{+}$-highest weight of $F$. Then $\phi_{s}(F)$ determines $F$ up to isomorphism.

3. Suppose $H$ is any other real Cartan subgroup of $G$, and $R^{+}$is any set of positive roots for $R(\mathfrak{g}, \mathfrak{h})$. Write

$$
i\left(R_{s}^{+}, R^{+}\right): \mathfrak{h}_{s}^{*} \rightarrow \mathfrak{h}^{*}
$$

for the unique isomorphism implemented by an element of $G(\mathbb{C})$ and carrying $R_{s}^{+}$to $R^{+}$. Then there is surjective group homomorphism

$$
I\left(R_{s}^{+}, R^{+}\right): \Lambda_{f i n}\left(H_{s}\right) \rightarrow \Lambda_{f i n}(H)
$$

characterized by

a) for every finite-dimensional representation $F$ of $G, I\left(R_{s}^{+}, R^{+}\right)$ carries $\Delta\left(F, H_{s}\right)$ bijectively to $\Delta(F, H)$, and

b) the differential of $I\left(R_{s}^{+}, R^{+}\right)$is $i\left(R_{s}^{+}, R^{+}\right)$.

4. The kernel of the homomorphism $I\left(R_{s}^{+}, R^{+}\right)$consists of the characters of $H_{s}$ trivial on $H_{s} \cap\left[G_{0} \cdot H\right]$.

For the proofs, we refer to [38], Section 0.4. (The description in Proposition 16.1(4) of $\Lambda_{\text {fin }}\left(G, H_{s}\right)$ is not proved in [38], but we will make no use of it.)

Definition 16.3 ([38], Definition 7.2.5). Suppose $H$ is a Cartan sub$\{$ def $: \operatorname{cohf} a m\}$ group of $G, \Lambda_{\text {fin }}(G, H) \subset \widehat{H}$ is the group of weights of finite-dimensional representations, and $\lambda \in \mathfrak{h}^{*}$ is any weight. Write

$$
\lambda+\Lambda_{\mathrm{fin}}(G, H)=\left\{\lambda+\psi \mid \psi \in \Lambda_{\mathrm{fin}}(G, H)\right\},
$$

(a set of formal symbols), called the translate of $\Lambda_{f i n}(G, H)$ by $\lambda$. A coherent family of virtual $(\mathfrak{g}, K)$-modules based on $\lambda+\Lambda_{f i n}(G, H)$ is a map

$$
\Phi: \lambda+\Lambda_{\text {fin }}(G, H) \rightarrow \mathcal{G}(\mathfrak{g}, K)
$$

(notation as in Proposition 15.8) satisfying

a) $\Phi(\lambda+\psi)$ has infinitesimal character $\lambda+d \psi \in \mathfrak{h}^{*}$; and 
b) for every finite-dimensional representation $F$ of $G$,

$$
F \otimes \Phi(\lambda+\psi)=\sum_{\mu \in \Delta(F, H)} \Phi(\lambda+(\psi+\mu)) .
$$

In this last formula we regard $\Delta(F, H)$ as a multiset, in which $\mu$ occurs with multiplicity equal to the dimension of the $\mu$ weight space of $F$.

Definition 16.4. Suppose $\mathfrak{h} \subset \mathfrak{g}$ is a Cartan subalgebra of a complex $\{$ def : introots $\}$ reductive Lie algebra, and $\xi \in \mathfrak{h}^{*}$. The set of integral roots for $\xi$ is

$$
R(\xi)=\left\{\alpha \in R(\mathfrak{g}, \mathfrak{h}) \mid \xi\left(\alpha^{\vee}\right) \in \mathbb{Z}\right\} .
$$

Fix a positive root system $R^{+}(\xi) \subset R(\xi)$. We say that $\xi$ is integrally dominant if

$$
\xi\left(\alpha^{\vee}\right) \geq 0, \quad\left(\alpha \in R^{+}(\xi)\right) .
$$

If $R^{+}(\xi)$ is extended to a positive system $R^{+}$for $R(\mathfrak{g}, \mathfrak{h})$, then integral dominance is equivalent to

$$
\xi\left(\beta^{\vee}\right) \text { is not a strictly negative integer }\left(\beta \in R^{+}\right) .
$$

Occasionally we will need to make use of a stronger condition. We say that $\xi$ is real dominant for $R^{+}$if

$$
\xi\left(\beta^{\vee}\right) \in \mathbb{R}^{\geq 0} \quad\left(\beta \in R^{+}\right) .
$$

$\{$ eq:realdom $\}$

The integral Weyl group for $\xi$ is

$$
W(\xi)={ }_{\operatorname{def}} W(R(\xi)) \subset W(\mathfrak{g}, \mathfrak{h}) .
$$

An equivalent definition (by Chevalley's theorem for the affine Weyl group) is

$$
W(\xi)=\{w \in W(\mathfrak{g}, \mathfrak{h}) \mid w \xi-\xi \in \mathbb{Z} R(\mathfrak{g}, \mathfrak{h})\} .
$$

([38], Lemma 7.2.17).

It is useful also to define the set of singular roots for $\xi$

$$
R^{\xi}=\left\{\alpha \in R(\mathfrak{g}, \mathfrak{h}) \mid \xi\left(\alpha^{\vee}\right)=0\right\} \subset R(\xi) .
$$

Notice that the choice of a system of positive integral roots making $\xi$ integrally dominant is precisely the same as the choice of an (arbitrary) system of positive singular roots $R^{\xi}{ }^{+}$. The correspondence is

$$
\begin{aligned}
R^{+}(\xi) & =\left\{\alpha \in R(\xi) \mid \xi\left(\alpha^{\vee}\right)>0\right\} \cup R^{\xi,+}, \\
R^{\xi,+} & =R^{+}(\xi) \cap R^{\xi} .
\end{aligned}
$$


In the same way, if $\xi$ is real

The singular Weyl group for $\xi$ is

$$
W^{\xi}=_{\text {def }} W\left(R^{\xi}\right) \subset W(R(\xi)) \subset W(\mathfrak{g}, \mathfrak{h}) .
$$

An equivalent definition (by Chevalley's theorem for $W$ ) is

$$
W^{\xi}=\{w \in W(\mathfrak{g}, \mathfrak{h}) \mid w \xi=\xi\}
$$

In terms of the notion of integral roots, we can recast Proposition 16.2 in a way that is often useful. So fix two Cartan subalgebras

$$
\mathfrak{h}_{1}, \mathfrak{h}_{2} \subset \mathfrak{g},
$$

and weights

$$
\lambda_{i} \in \mathfrak{h}_{i}^{*} \quad(i=1,2) .
$$

Assume now that

$$
\xi_{\lambda_{1}}=\xi_{\lambda_{2}}
$$

equivalently, that there is an isomorphism $\mathfrak{h}_{1}^{*} \simeq \mathfrak{h}_{2}^{*}$ implemented by an element of $G(\mathbb{C})$ and carrying $\lambda_{1}$ to $\lambda_{2}$ :

$$
i: \mathfrak{h}_{1}^{*} \rightarrow \mathfrak{h}_{2}^{*}, \quad i\left(\lambda_{1}\right)=\lambda_{2} .
$$

This isomorphism is unique only up to (multiplication on the right by) the stabilizer $W^{\lambda_{1}}$ of $\lambda_{1}$. But if we fix also systems of positive singular roots

$$
R^{\lambda_{1},+}, \quad R^{\lambda_{2},+}
$$

then we can specify a unique isomorphism by requiring

$$
i\left(\lambda_{1}, R^{\lambda_{1},+}, \lambda_{2}, R^{\lambda_{2},+}\right): \mathfrak{h}_{1}^{*} \rightarrow \mathfrak{h}_{2}^{*}, \quad \lambda_{1} \mapsto \lambda_{2}, \quad R^{\lambda_{1},+} \mapsto R^{\lambda_{2},+} .
$$

Equivalently, if we require that $\lambda_{i}$ is integrally dominant for $R^{+}\left(\lambda_{i}\right)$, then we can specify a unique isomorphism

$$
\begin{aligned}
i\left(\lambda_{1}, R^{+}\left(\lambda_{1}\right), \lambda_{2}, R^{+}\left(\lambda_{2}\right)\right) & : \mathfrak{h}_{1}^{*} \rightarrow \mathfrak{h}_{2}{ }^{*}, \\
\lambda_{1} & \mapsto \lambda_{2}, \quad R^{+}\left(\lambda_{1}\right) \mapsto R^{+}\left(\lambda_{2}\right) .
\end{aligned}
$$

These isomorphisms can take the place of $i\left(R_{s}^{+}, R^{+}\right)$in Proposition 16.2. 
Theorem 16.6 ([38], Corollary 7.3.23). Suppose $\Gamma=\left(H, \gamma, R_{i \mathbb{R}}^{+}\right)$is a Lang$\{$ thm: cohfam $\}$ lands parameter for $G$, and $J(\Gamma)$ the corresponding irreducible representation (Theorem 6.1). Write $d \gamma \in \mathfrak{h}^{*}$ for the differential of $\gamma$, which is the infinitesimal character of $J(\Gamma)$. Write $R(d \gamma)$ for the set of integral roots (Definition 16.4). Then $R(d \gamma)$ is automatically preserved by the action of the Cartan involution $\theta$.

Fix a set $R^{+}(d \gamma)$ of positive integral roots, subject to the following requirements:

a) if $d \gamma\left(\alpha^{\vee}\right)$ is a positive integer, then $\alpha \in R^{+}(d \gamma)$;

b) if $d \gamma\left(\alpha^{\vee}\right)=0$, but $d \gamma\left(\theta \alpha^{\vee}\right)>0$, then $\alpha \in R^{+}(d \gamma)$; and

c) $R^{+}(d \gamma) \supset R_{i \mathbb{R}}^{+}$.

Fix a set $R^{+}$of positive roots for $H$ in $G$ containing $R^{+}(d \gamma)$. Suppose finally that $H_{s}$ is a maximally split Cartan, with any positive root system $R_{s}^{+}$, as in Proposition 16.2; we will use the maps $i\left(R_{s}^{+}, R^{+}\right)$and $I\left(R_{s}^{+}, R^{+}\right)$defined in that proposition. Set

$$
d \gamma_{s}=i\left(R_{s}^{+}, R^{+}\right)^{-1}(d \gamma) \in \mathfrak{h}_{s}^{*}, \quad R^{+}\left(d \gamma_{s}\right)=i\left(R_{s}^{+}, R^{+}\right)^{-1}\left(R^{+}(d \gamma)\right) .
$$

Then there is a unique coherent family $\Phi$ of virtual $(\mathfrak{g}, K)$-modules based on $d \gamma_{s}+\Lambda_{f i n}\left(G, H_{s}\right)$, with the following characteristic properties:

a) $\Phi\left(d \gamma_{s}\right)=[J(\Gamma)]$; and

b) whenever $d \gamma_{s}+d \psi_{s}$ is integrally dominant (with respect to $R^{+}\left(d \gamma_{s}\right)$ ), $\Phi(d \gamma+\psi)$ is (the class in the Grothendieck group of) an irreducible representation or zero.

Write $\Pi(d \gamma)$ for the set of simple roots of $R^{+}(d \gamma)$, and define a subset $\tau(\Gamma) \subset \Pi(d \gamma)$ by

a) if $\alpha \in \Pi(d \gamma)$ is real, (Definition 5.7), then $\alpha \in \tau(\Gamma)$ if and only if $\gamma_{\mathfrak{q}}\left(m_{\alpha}\right)=(-1)^{d \gamma\left(\alpha^{\vee}\right)+1}$ (notation $\left.(9.3 \mathrm{~d})\right)$;

b) if $\beta \in \Pi(d \gamma)$ is imaginary, (Definition 5.7), then $\alpha \in \tau(\Gamma)$ if and only if $\beta$ is compact; and

c) if $\delta \in \Pi(d \gamma)$ is complex, (Definition 5.7), then $\delta \in \tau(\Gamma)$ if and only if $\theta \delta$ is a negative root. 
Write

$$
\tau_{s}(\Gamma)=i\left(R_{s}^{+}, R^{+}\right)^{-1}\left(\tau(\Gamma) \subset \Pi\left(d \gamma_{s}\right)\right.
$$

for the corresponding integral simple roots for $H_{s}$. The coherent family $\Phi$ has the following additional properties.

1. If $d \gamma_{s}+d \psi_{s}$ is integrally dominant, then $\Phi\left(d \gamma_{s}+\psi_{s}\right)=0$ if and only if there is a simple root $\alpha_{s} \in \tau_{s}(\Gamma)$ with $(d \gamma+d \psi)\left(\alpha_{s}^{\vee}\right)=0$.

2. Suppose that $d \gamma_{s}+d \psi_{s}$ is integrally dominant, and does not vanish on any root in $\tau_{s}(\Gamma)$. Write

$$
\psi=I\left(R_{s}^{+}, R^{+}\right)\left(\psi_{s}\right) \in \Lambda_{f i n}(H) .
$$

Then

$$
\Gamma+\psi={ }_{\operatorname{def}}\left(H, \gamma+\psi, R_{i \mathbb{R}}^{+}\right)
$$

is a Langlands parameter; and

$$
\Phi\left(d \gamma_{s}+\psi_{s}\right)=[J(\Gamma+\psi)]
$$

3. The set of irreducible constituents of the various virtual representations $\Psi\left(d \gamma_{s}+\psi_{s}\right)$ is equal to the set of irreducible constituents of all the tensor products $F \otimes J(\Gamma)$, with $F$ a finite-dimensional representation of $G$.

Definition 16.7. In the setting of Theorem 16.6, the subset

$$
\tau_{s}(\Gamma) \subset \Pi\left(d \gamma_{s}\right)
$$

of the simple integral roots is called the $\tau$-invariant of the irreducible representation $J(\Gamma)$, or the $\tau$-invariant of $\Gamma$. (Because of the canonical isomorphism $i\left(R_{s}^{+}, R^{+}\right)$, we may also refer to $\tau(\Gamma) \subset \Pi(d \gamma)$ as the $\tau$ invariant.)

Return for a moment to the abstract language of (15.1). So we fix a $\{$ se: transfunc\} maximally split Cartan $H_{s}$ of $G$, and a weight $\lambda_{0} \in \mathfrak{h}_{s}^{*}$ corresponding to a (possibly singular) infinitesimal character

$$
\xi_{\lambda_{0}}: \mathfrak{Z}(\mathfrak{g}) \rightarrow \mathbb{C}
$$

Write $R\left(\lambda_{0}\right)$ for the integral roots (Definition 16.4), and choose a set of positive roots

$$
R^{+}\left(\lambda_{0}\right) \subset R\left(\lambda_{0}\right) \subset R\left(\mathfrak{g}, \mathfrak{h}_{s}\right)
$$


making $\lambda_{0}$ integrally dominant. When we study signatures of invariant forms, we be assuming that the infinitesimal character is real (Definition $5.5)$, and in that case we can extend $R^{+}\left(\lambda_{0}\right)$ uniquely to a positive system

$$
R_{s}^{+} \supset R^{+}\left(\lambda_{0}\right)
$$

\{eq: lambda0realdom $\}$

making $\lambda_{0}$ real dominant $(16.4 \mathrm{~d})$.

It will be useful to have notation for the simple roots

$$
\Pi\left(\lambda_{0}\right) \subset R^{+}\left(\lambda_{0}\right)
$$

and for the subset

$$
\left\{\alpha \in \Pi\left(\lambda_{0}\right) \mid \lambda_{0}\left(\alpha^{\vee}\right)=0\right\}={ }_{\operatorname{def}} \Pi^{\lambda_{0}}
$$

of simple roots orthogonal to $\lambda_{0}$. Finally, we fix a weight

$$
\phi \in \Lambda_{\mathrm{fin}}\left(G, H_{s}\right)
$$

of a finite-dimensional representation of $G$, subject to the additional condition

$$
\lambda_{0}+d \phi \text { is dominant and regular for } R^{+}\left(\lambda_{0}\right) .
$$

In connection with signatures of invariant forms, we will want sometimes to assume also that

$$
\lambda_{0}+d \phi \text { is dominant and regular for } R_{s}^{+} .
$$

One way to achieve this is to take $\phi$ to be the sum of the roots in $R_{s}^{+}$. We need also

$$
F_{\phi}=\text { finite-dimensional irreducible of extremal weight } \phi .
$$

In order to define the Jantzen-Zuckerman translation functors, we need the functor (on $\mathfrak{g}$-modules)

$$
P_{\lambda_{0}}(M)=\begin{aligned}
& \text { largest submodule of } M \text { on which } z-\xi_{\lambda_{0}}(z) \\
& \text { acts nilpotently, all } z \in \mathfrak{Z}(\mathfrak{g})
\end{aligned}
$$

If $M$ is a $(\mathfrak{g}, K)$ module, so is $P_{\lambda_{0}}(M)$. On the category of $\mathfrak{Z}(\mathfrak{g})$-finite modules (for example, on $(\mathfrak{g}, K)$-modules of finite length) the functor $P_{\lambda_{0}}$ is exact. The translation functors we want are

$$
T_{\lambda_{0}}^{\lambda_{0}+\phi}(M)=_{\text {def }} P_{\lambda_{0}+d \phi}\left(F_{\phi} \otimes P_{\lambda_{0}}(M)\right),
$$


("translation away from the wall") and

$$
T_{\lambda_{0}+\phi}^{\lambda_{0}}(N)={ }_{\text {def }} P_{\lambda_{0}}\left(F_{\phi}^{*} \otimes P_{\lambda_{0}+d \phi}(N)\right)
$$

("translation to the wall.") These are clearly exact functors on the category of finite-length $(\mathfrak{g}, K)$-modules, so they also define group endomorphisms of the Grothendieck group $\mathcal{G}(\mathfrak{g}, K)$.

Corollary 16.9 (Jantzen [16], Zuckerman [47]; see [38], Proposition 7.2.22). $\{$ cor:transfunc $\}$ Suppose we are in the setting of (16.8).

1. The functors $T_{\lambda_{0}+\phi}^{\lambda_{0}}$ and $T_{\lambda_{0}}^{\lambda_{0}+\phi}$ are left and right adjoint to each other:

$$
\operatorname{Hom}_{\mathfrak{g}, K}\left(N, T_{\lambda_{0}}^{\lambda_{0}+\phi}(M)\right) \simeq \operatorname{Hom}_{\mathfrak{g}, K}\left(T_{\lambda_{0}+\phi}^{\lambda_{0}}(N), M\right),
$$

and similarly with the two functors interchanged.

2. For a coherent family $\Phi$ of virtual $(\mathfrak{g}, K)$-modules on $\lambda_{0}+\Lambda_{f i n}\left(G, H_{s}\right)$,

$$
T_{\lambda_{0}+\phi}^{\lambda_{0}}\left(\Phi\left(\lambda_{0}+\phi\right)\right)=\Phi\left(\lambda_{0}\right) .
$$

3. The translation functor $T_{\lambda_{0}+\phi}^{\lambda_{0}}$ (translation to the wall) carries every irreducible $(\mathfrak{g}, K)$-module of infinitesimal character $\lambda_{0}+d \phi$ to an irreducible of infinitesimal character $\lambda_{0}$, or to zero. The irreducible representations mapped to zero are exactly those containing some root of $\Pi^{\lambda_{0}}$ in the $\tau$-invariant (Definition 16.7).

4. The (inverse of the) translation functor of (3) defines an injective correspondence of Langlands parameters (and therefore of irreducible representations)

$$
t_{\lambda_{0}}^{\lambda_{0}+\phi}: B\left(\lambda_{0}\right) \hookrightarrow B\left(\lambda_{0}+d \phi\right) .
$$

The image consists of all irreducible representations (of infinitesimal character $\lambda_{0}+d \phi$ ) having no root of $\Pi^{\lambda_{0}}$ in the $\tau$-invariant.

5. Suppose $\Gamma_{0}=\left(H, \gamma_{0}, R_{i \mathbb{R}}^{+}\right) \in B\left(\lambda_{0}\right)$ is a Langlands parameter (at the possibly singular infinitesimal character $\left.\lambda_{0}\right)$. Choose positive integral roots $R^{+}\left(d \gamma_{0}\right)$ as in Theorem 16.6, so that we have an isomorphism

$$
i\left(\lambda_{0}, R^{+}\left(\lambda_{0}\right), d \gamma_{0}, R^{+}\left(d \gamma_{0}\right)\right): \mathfrak{h}_{s}^{*} \rightarrow \mathfrak{h}^{*}
$$

as in (16.5). Define

$$
\phi\left(\Gamma_{0}\right)=I\left(\lambda_{0}, R^{+}\left(\lambda_{0}\right), d \gamma_{0}, R^{+}\left(d \gamma_{0}\right)\right)(\phi),
$$


an $\mathrm{H}$-weight of a finite-dimensional representation of $G$ (Proposition 16.2). Then the correspondence of Langlands parameters in (4) is

$$
t_{\lambda_{0}}^{\lambda_{0}+\phi}\left(\Gamma_{0}\right)=\Gamma_{0}+\phi\left(\Gamma_{0}\right)
$$

(notation as in Theorem 16.6(4)).

6. Suppose $\Gamma_{1}=\left(H, \gamma_{1}, R_{i \mathbb{R}}^{+}\right) \in B\left(\lambda_{0}+d \phi\right)$ is a Langlands parameter (at the regular infinitesimal character $\left.\lambda_{0}+d \phi\right)$. Write $R^{+}\left(d \gamma_{1}\right)$ for the unique set of positive integral roots making $d \gamma_{1}$ integrally dominant, so that we have an isomorphism

$$
i\left(\lambda_{0}+d \phi, R^{+}\left(\lambda_{0}\right), d \gamma_{1}, R^{+}\left(d \gamma_{1}\right)\right): \mathfrak{h}_{s}^{*} \rightarrow \mathfrak{h}^{*}
$$

as in (16.5). Define

$$
\phi\left(\Gamma_{1}\right)=I\left(\lambda_{0}, R^{+}\left(\lambda_{0}\right), d \gamma_{0}, R^{+}\left(d \gamma_{0}\right)\right)(\phi),
$$

an $\mathrm{H}$-weight of a finite-dimensional representation of $G$ (Proposition 16.2). Then

$$
t_{\lambda_{0}+\phi}^{\lambda_{0}}\left(\Gamma_{1}\right)=_{\text {def }}\left(H, \gamma_{1}-\phi\left(\Gamma_{1}\right), R_{i \mathbb{R}}^{+}\right)=_{\text {def }} \Gamma_{1}-\phi\left(\Gamma_{1}\right)
$$

is a weak Langlands parameter (Definition 6.3). This correspondence is a left inverse to the injection of (4):

$$
t_{\lambda_{0}+\phi}^{\lambda_{0}} \circ t_{\lambda_{0}}^{\lambda_{0}+\phi}\left(\Gamma_{0}\right)=\Gamma_{0}
$$

7. Translation to the wall carries standard representations to weak standard representations:

$$
T_{\lambda_{0}+\phi}^{\lambda_{0}}\left(I_{\text {quo }}\left(\Gamma_{1}\right)=I_{\text {quo }}\left(\Gamma_{1}-\phi\left(\Gamma_{1}\right)\right) .\right.
$$

In particular,

$$
T_{\lambda_{0}+\phi}^{\lambda_{0}}\left(I_{\mathrm{quo}}\left(\Gamma_{0}+\phi\left(\Gamma_{0}\right)\right)=I_{\mathrm{quo}}\left(\Gamma_{0}\right) .\right.
$$

8. Translation to the wall carries irreducibles to irreducibles or to zero:

$$
T_{\lambda_{0}+\phi}^{\lambda_{0}}\left(J\left(\Gamma_{1}\right)= \begin{cases}J\left(\Gamma_{1}-\phi\left(\Gamma_{1}\right)\right) & \left(\tau\left(J\left(\Gamma_{1}\right)\right) \cap \Pi^{\lambda_{0}}=\emptyset\right), \\ 0 & \left(\tau\left(J\left(\Gamma_{1}\right)\right) \cap \Pi^{\lambda_{0}} \neq \emptyset\right) .\end{cases}\right.
$$

In particular,

$$
T_{\lambda_{0}+\phi}^{\lambda_{0}}\left(J\left(\Gamma_{0}+\phi\left(\Gamma_{0}\right)\right)=J\left(\Gamma_{0}\right) .\right.
$$


Corollary 16.9 allows us to "reduce" the computation of the multiplicity \{se:reducetoreg\} matrix $m_{\Xi, \Gamma}$ defined in (15.1) to the case of regular infinitesimal character. Given a possibly singular infinitesimal character $\lambda_{0}$, we choose a weight of a finite-dimensional representation $\phi$ as in (16.8), so that $\lambda_{0}+d \phi$ is regular. The (possibly singular) Langlands parameters $\Xi$ and $\Gamma$ in $B\left(\lambda_{0}\right)$ correspond to regular Langlands parameters $\Xi+\phi(\Xi)$ and $\Gamma+\phi(\Gamma)$ in $B\left(\lambda_{0}+d \phi\right)$, and

$$
m_{\Xi, \Gamma}=m_{\Xi+\phi(\Xi), \Gamma+\phi(\Gamma)} .
$$

A little more precisely, we can start with the decomposition into irreducibles

$$
[I(\Gamma+\phi(\Gamma))]=\sum_{\Xi_{1} \in B\left(\lambda_{0}+d \phi\right)} m_{\Xi_{1}, \Gamma+\phi(\Gamma)}\left[J\left(\Xi_{1}\right)\right]
$$

and apply the exact translation functor $T_{\lambda_{0}+\phi}^{\lambda_{0}}$. On the left we get $[I(\Gamma)]$, by Corollary 16.9(7). On the right we get

$$
\sum_{\substack{\Xi_{1} \in B\left(\lambda_{0}+d \phi\right) \\ \tau\left(\Xi_{1}\right) \cap \Pi^{\lambda_{0}}=\emptyset}} m_{\Xi_{1}, \Gamma+\phi(\Gamma)}\left[J\left(\Xi_{1}-\phi\left(\Xi_{1}\right)\right)\right] .
$$

The irreducibles appearing on the right are all distinct (this is part of the uniqueness assertion for the coherent family in Theorem 16.6), so the coefficients are the multiplicities of irreducibles in $I(\Gamma)$.

To say this another way: the index set of Langlands parameters at the possibly singular infinitesimal character $\lambda_{0}$ is naturally a subset of the index set at the regular infinitesimal character $\lambda_{0}+d \phi$. The multiplicity matrix at $\lambda_{0}$ is the corresponding submatrix of the one at $\lambda_{0}+d \phi$.

We call this fact a "reduction" in quotes, because the multiplicity problem at regular infinitesimal character is much more complicated than at singular infinitesimal character: we have "reduced" a less complicated problem to a more complicated one. But the gain is real. At regular infinitesimal character we have the Kazhdan-Lusztig algorithm (see Section 18) to solve the problem, and this algorithm cannot (as far as we now understand) be applied directly to the case of singular infinitesimal character.

Finally, let us consider the problem of relating the character matrix $M_{\Xi, \Gamma}$ to regular infinitesimal character. Since this problem concerns the inverse of the multiplicity matrix, we might suspect that there are difficulties: there is not usually a nice way to find the inverse of a submatrix from the inverse of the larger matrix. Since our matrices are upper triangular, one could hope that the submatrix we are considering is a corner; but that turns 
out not to be the case. In order to see what happens, we begin with a careful statement of what happens to standard modules under translation to singular infinitesimal character.

Theorem 16.11. Suppose we are in the setting of (16.8). Write

$$
t_{\lambda_{0}+\phi}^{\lambda_{0}}: B\left(\lambda_{0}+d \phi\right) \rightarrow B_{\text {weak }}\left(\lambda_{0}\right)
$$

(notation (15.1)) for the map of Corollary 16.9(7). Fix a Langlands parameter $\Xi_{1} \in B\left(\lambda_{0}+d \phi\right)$. Write

$$
\Xi_{0}=t_{\lambda_{0}+\phi}^{\lambda_{0}}\left(\Xi_{1}\right) \in B_{\text {weak }}\left(\lambda_{0}\right)
$$

so that

$$
T_{\lambda_{0}+\phi}^{\lambda_{0}}\left(I_{\mathrm{quo}}\left(\Xi_{1}\right)\right)=I_{\mathrm{quo}}\left(\Xi_{0}\right) .
$$

1. There is a unique subset $C_{\text {sing }}\left(\Xi_{0}\right) \subset B\left(\lambda_{0}\right)$ with the property that

$$
I_{\text {quo }}\left(\Xi_{0}\right)=\sum_{\Xi^{\prime} \in C_{\text {sing }}\left(\Xi_{0}\right)} I_{\text {quo }}\left(\Xi^{\prime}\right)
$$

2. The set $C_{\text {sing }}\left(\Xi_{0}\right)$ has cardinality either equal to zero (in case some simple compact imaginary root for $\Xi_{0}$ vanishes on $\lambda_{0}$, so that $I\left(\Xi_{0}\right)=$ 0) or a power of two.

For each Langlands parameter $\Xi \in B\left(\lambda_{0}\right)$, define

$$
C_{\text {reg }}(\Xi)=\left\{\Xi_{1} \in B\left(\lambda_{0}+d \phi\right) \mid \Xi \in C_{\text {sing }}\left(\Xi_{0}\right)\right\} \subset B\left(\lambda_{0}+d \phi\right) ;
$$

here $\Xi_{0}=t_{\lambda_{0}+d \phi}^{\lambda_{0}}\left(\Xi_{1}\right)$ as above.

3. Suppose $\Gamma \in B\left(\lambda_{0}\right)$ is any Langlands parameter, corresponding to $\Gamma+$ $\phi(\Gamma) \in B\left(\lambda_{0}\right)$. Then the character formula (15.11) is

$$
J(\Gamma)=\sum_{\Xi \in B\left(\lambda_{0}\right)} \sum_{\Xi_{1} \in C_{r e g}(\Xi)} M_{\Xi_{1}, \Gamma+\phi(\Gamma)}[I(\Xi)] .
$$

Equivalently,

$$
M_{\Xi, \Gamma}=\sum_{\Xi_{1} \in C_{r e g}(\Xi)} M_{\Xi_{1}, \Gamma+\phi(\Gamma)} .
$$


Sketch of proof. That $\Xi_{0}$ must be a weak Langlands parameter is Corol\{se:reducechartoreg\} lary $16.9(6)$, and the statement about translation of standard modules is Corollary $16.9(7)$. As is explained in (6.4), the weak standard module $I\left(\Xi_{0}\right)$ of infinitesimal character $\lambda_{0}$ is either equal to zero (in case the parameter vanishes on some simple compact imaginary root) or can be written (by a simple algorithm) as a direct sum

$$
I\left(\Xi_{0}\right)=\sum_{\Xi^{\prime} \in C_{\text {sing }}\left(\Xi_{0}\right) \subset B\left(\lambda_{0}\right)} I\left(\Xi^{\prime}\right) .
$$

The letter $C$ stands for "Cayley." The fundamental example is writing a nonspherical principal series for $S L(2, \mathbb{R})$ (with continuous parameter zero) as a direct sum of two limits of discrete series; the general case is just a repeated application of this one.) The construction of this decomposition takes place inside the subgroup of $G$ generated by the Cartan subgroup $H_{1}$ for the parameter $\Xi_{0}$, and the real roots in $\Pi^{\xi_{0}}$. It turns out that the parameters appearing in $C_{\text {sing }}\left(\Xi_{0}\right)$ are all attached to a single Cartan subgroup, and that the number of them is a power of 2 . Since we will not use these last two facts, we omit the proof. This is (2).

For the character formula in (3), we begin with the character formula at infinitesimal character $\lambda_{0}+d \phi$ :

$$
[J(\Gamma+\phi(\Gamma))]=\sum_{\Xi_{1} \in B\left(\lambda_{0}+d \phi\right)} M_{\Xi_{1}, \Gamma+\phi(\Gamma)}\left[I\left(\Xi_{1}\right)\right]
$$

Now apply the exact translation functor $T_{\lambda_{0}+\phi}^{\lambda_{0}}$. On the left we get $[J(\Gamma)]$, by Corollary 16.9(8). On the right we get a sum of weak standard modules

$$
\sum_{\Xi_{1} \in B\left(\lambda_{0}+d \phi\right)} M_{\Xi_{1}, \Gamma+\phi(\Gamma)}\left[I\left(\Xi_{1}-\phi\left(\Xi_{1}\right)\right)\right]=\sum_{\Xi_{1} \in B\left(\lambda_{0}+d \phi\right)} M_{\Xi_{1}, \Gamma+\phi(\Gamma)}\left[I\left(\Xi_{0}\right)\right] .
$$

Using (2), we may therefore rewrite our character formula as

$$
\begin{aligned}
{[J(\Gamma)] } & =\sum_{\Xi \in B\left(\lambda_{0}\right)} \sum_{\substack{\Xi_{1} \in B\left(\lambda_{0}+d \phi\right) \\
\Xi \in C_{\mathrm{sing}}\left(\Xi_{0}\right)}} M_{\Xi_{1}, \Gamma+\phi(\Gamma)}[I(\Xi)] \\
& =\sum_{\Xi \in B\left(\lambda_{0}\right)} \sum_{\Xi_{1} \in C_{\mathrm{reg}}(\Xi)} M_{\Xi_{1}, \Gamma+\phi(\Gamma)}[I(\Xi)] .
\end{aligned}
$$

Equivalently,

$$
M_{\Xi, \Gamma}=\sum_{\Xi_{1} \in C_{\mathrm{reg}}(\Xi)} M_{\Xi_{1}, \Gamma+\phi(\Gamma)} .
$$


It is natural to ask about a corresponding use of the translation functors to relate the formulas (15.12) at two different infinitesimal characters. More or less this is exactly parallel to the discussion above, but there is a subtlety about the choice of invariant form: the translation functor will carry one nondegenerate invariant form to another, but the new form may not share the characteristic positivity property of the old one. We therefore postpone a careful analysis to Section 20.

\section{Translation functors for extended groups}

In this section we fix an extended group

$$
{ }^{\delta} G=G \rtimes\left\{1, \delta_{f}\right\}
$$

as in Definition 12.3. Suppose

$$
{ }^{1} H=\left\langle H, \delta_{1}\right\rangle, \quad t_{1}\left(R_{1}^{+}\right)=R_{1}^{+}
$$

is an extended maximal torus preserving a positive root system $R_{1}^{+}$, with $\delta_{1} \in K$ a representative for the element $t_{1}$ of the extended Weyl group. According to Clifford theory (Proposition 8.13),

- each $\gamma=\gamma^{t_{1}} \in \widehat{H}$ has two one-dimensional extensions $\gamma_{ \pm}$to

${ }^{1} H$, on which the scalar actions of $\delta_{1}$ differ by sign;

- each $\gamma \neq \gamma^{t_{1}} \in \widehat{H}$ induces a two-dimensional irreducible $\gamma_{\text {ind }}=\gamma_{\text {ind }}^{t_{1}} \in \widehat{{ }^{1} H}$; and

\{eq:exttorusreps\}

- these types exhaust $\widehat{{ }^{1} H}$.

Of course it also makes sense to induce a $t_{1}$-fixed character $\gamma$; in that case we get

$$
\gamma_{\text {ind }}=\gamma_{+}+\gamma_{-} \quad\left(\gamma=\gamma^{t_{1}}\right) .
$$

Despite the notation, there is no way to prefer one of the two extensions $\gamma+$ and $\gamma_{-}$without making a further choice: that of a square root of the scalar by which $\delta_{1}^{2} \in H$ acts in $\gamma$. 
There is an exactly parallel description of the finite-dimensional representations of ${ }^{\delta} G$ :

- each $t_{1}$-fixed (equivalently, $\theta$-fixed) finite-dimensional irreducible representation $F$ of $G$ has two extensions $F_{ \pm}$to ${ }^{\delta} G$, distinguished by the action of $\delta_{1}$ on the $R_{1}^{+}$-highest weight space;

- each member of a two-element orbit $\left\{F, F^{t_{1}}\right\}$ of irreducible $\{$ eq:extGfindiml\} finite-dimensional representations of $G$ induces to an irreducible representation $F_{\text {ind }}=F_{\text {ind }}^{t_{1}}$ of ${ }^{\delta} G$; and

- these two types exhaust the irreducible finite-dimensional representations of ${ }^{\delta} G$.

Inducing a $\theta$-fixed finite-dimensional irreducible to ${ }^{\delta} G$ gives

$$
F_{\text {ind }}=F_{+}+F_{-} \quad\left(F=F^{t_{1}}\right) .
$$

Again the notation obscures that fact that there is no way to prefer $F_{+}$ over $F_{-}$without choosing a square root of the action of $\delta_{1}^{2}$ on the highest weight space.

There is a possibly unexpected subtlety about highest weight theory for ${ }^{\delta} G$ : the highest weight of an irreducible finite-dimensional representation need not be an irreducible representation of ${ }^{1} \mathrm{H}$, and need not determine the representation of ${ }^{\delta} G$. For us, the most important aspect of this problem is that the highest weight of $F$ may be $t_{1}$-fixed even if $F$ is not $\theta$-fixed. Examples are provided by Example 17.3 below.

Following Proposition 16.1, for each finite-dimensional representation $F_{1}$ of ${ }^{\delta} G$, define

$$
\Delta\left(F_{1},{ }^{1} H\right) \subset \widehat{{ }^{1} H}
$$

to be the collection of irreducible representations of ${ }^{1} H$ appearing in the restriction of $F_{1}$. Sometimes it will be convenient to regard $\Delta\left(F_{1},{ }^{1} H\right)$ as a multiset. We define

$$
\Lambda_{\text {fin }}\left({ }^{\delta} G,{ }^{1} H\right) \subset \widehat{{ }^{1} H}
$$

to be the set of irreducible representations of ${ }^{1} H$ appearing in the restrictions of finite-dimensional representations of ${ }^{\delta} G$. Using finite-dimensional representations induced from $G$ to ${ }^{\delta} G$, it is very easy to see that

$$
\Lambda_{\text {fin }}\left({ }^{\delta} G,{ }^{1} H\right)=\left\{\phi_{1} \in \widehat{{ }^{1} H}\left|\phi_{1}\right|_{H} \subset \Lambda_{\text {fin }}(G, H)\right\} ;
$$


here $\subset$ indicates that the restriction is a sum of characters which are weights of finite-dimensional representations of $G$. We write

$$
\mathcal{G}_{\text {fin }}\left({ }^{1} H\right)=\text { subgroup of } \mathcal{G}\left({ }^{1} H\right) \text { generated by } \Lambda_{\text {fin }}\left({ }^{\delta} G,{ }^{1} H\right) .
$$

Finally, for any finite-dimensional representation of $F_{1}$ of ${ }^{\delta} G$, define

$$
\phi_{1}\left(F_{1}\right)=\text { representation of }{ }^{1} H \text { on } R_{1}^{+} \text {-highest weight vectors. }
$$

Even if $F_{1}$ is irreducible, it turns out that $\phi_{1}\left(F_{1}\right)$ need not be irreducible; this is related again to the phenomenon in Example 17.3.

Proposition 17.2. In the setting of Proposition 16.2, suppose that $R_{s}^{+}$is \{prop:findimlextHswts\} chosen compatible with an extended maximal torus ${ }^{1} H_{s}$, and that $t_{s}$ is the corresponding automorphism of $H_{s}$.

1. The surjection

$$
I\left(R_{s}^{+}, R_{1}^{+}\right): \Lambda_{f i n}\left(H_{s}\right) \rightarrow \Lambda_{f i n}(H)
$$

carries the action of $t_{s}$ to that of $t_{1}$.

2. The surjection induces a homomorphism of Grothendieck groups

$$
{ }^{\delta} I\left(R_{s}^{+}, R_{1}^{+}\right): \mathcal{G}_{\text {fin }}\left({ }^{1} H_{s}\right) \rightarrow \mathcal{G}_{\text {fin }}\left({ }^{1} H\right)
$$

preserving dimensions and satisfying

(a) For every finite-dimensional representation $F_{1}$ of ${ }^{\delta} G,{ }^{\delta} I\left(R_{s}^{+}, R_{1}^{+}\right)$ carries the restriction of $F_{1}$ to ${ }^{1} H_{s}$ to the restriction of $F_{1}$ to ${ }^{1} \mathrm{H}$.

(b) For every weight $\lambda_{s} \in \Lambda_{\text {fin }}\left({ }^{\delta} G,{ }^{1} H_{s}\right)$, write $\lambda=I\left(R_{s}^{+}, R_{1}^{+}\right)\left(\mu_{s}\right)$. Then

$$
{ }^{\delta} I\left(R_{s}^{+}, R_{1}^{+}\right)\left(\lambda_{s, i n d}\right)=\lambda_{\text {ind }} .
$$

(c) If in addition $\lambda_{s} \in \Lambda_{\text {fin }}^{t_{s}}\left({ }^{\delta} G,{ }^{1} H_{s}\right)$ is fixed by $t_{s}$, then automatically $\lambda$ is fixed by $t_{1}$; and

$$
{ }^{\delta} I\left(R_{s}^{+}, R_{1}^{+}\right)\left(\lambda_{s, \pm}\right)=\lambda_{ \pm}
$$

3. $A t_{s}$-fixed dominant weight $\lambda_{s} \in \Lambda_{\text {fin }}^{t_{s}}\left(G, H_{s}\right)$ is automatically the highest weight of unique a finite-dimensional irreducible representation $F\left(\lambda_{s}\right) \simeq F\left(\lambda_{s}\right)^{\theta}$. 
4. A $t_{1}$-fixed dominant weight $\lambda \in \Lambda_{\text {fin }}^{t_{1}}(G, H)$ is the highest weight of a $\theta$-fixed finite-dimensional representation of $G$ if and only if

$$
\lambda=I\left(R_{s}^{+}, R_{1}^{+}\right)\left(\lambda_{s}\right) \quad\left(\lambda_{s} \in \Lambda_{\text {fin }}^{t_{s}}\left(G, H_{s}\right)\right) .
$$

It is important to note in (4) that there can exist $t_{1}$-fixed weights of finite-dimensional representations that are not highest weights of $\theta$-fixed finite-dimensional representations of $G$.

Example 17.3. Suppose $G=G L(2, \mathbb{R})$. We take for $H$ the Cartan subgroup

$$
H \simeq \mathbb{C}^{\times}=\left\{r e^{i \phi} \mid r>0, \phi \in \mathbb{R}\right\},
$$

coming from the multiplicative action on $\mathbb{C} \simeq \mathbb{R}^{2}$. The Cartan involution (inverse transpose) acts by $\theta(z)=\bar{z}^{-1}$, so this is also the action of $t_{1}$ :

$$
t_{1}\left(r e^{i \phi}\right)=r^{-1} e^{i \phi}
$$

The characters of $H$ appearing in finite-dimensional representations are

$$
\lambda_{a, m}\left(r e^{i \phi}\right)=r^{2 \nu} e^{i m \phi} \quad(m \in \mathbb{Z}, \nu \in \mathbb{C}) .
$$

That is,

$$
\Lambda_{\text {fin }}(G, H) \simeq \mathbb{C} \times \mathbb{Z}, \quad t_{1}(\nu, m)=(-\nu, m) .
$$

The $t_{1}$-fixed weights are

$$
\Lambda_{\text {fin }}^{t_{1}}(G, H)=\{(0, m) \mid m \in \mathbb{Z}\} .
$$

For a maximally split Cartan we choose the diagonal subgroup

$$
H_{s} \simeq \mathbb{R}^{\times} \times \mathbb{R}^{\times}=\left\{\left(d_{1}, d_{2}\right) \mid d_{i} \in \mathbb{R}^{\times} .\right.
$$

The action of $t_{s}$ is

$$
t_{s}\left(d_{1}, d_{2}\right)=\left(d_{2}^{-1}, d_{1}^{-1}\right) .
$$

The characters appearing in finite-dimensional representations are

$$
\begin{aligned}
\lambda_{\nu, m, \delta}\left(d_{1}, d_{2}\right)= & \left|d_{1}\right|^{\nu+m / 2}\left|d_{2}\right|^{\nu-m / 2}\left(\operatorname{sgn} d_{1}\right)^{\delta}\left(\operatorname{sgn} d_{2}\right)^{m+\delta} \\
& (\nu \in \mathbb{C}, m \in \mathbb{Z}, \delta \in \mathbb{Z} / 2 \mathbb{Z}) .
\end{aligned}
$$

Therefore

$$
\Lambda_{\text {fin }}\left(G, H_{s}\right) \simeq \mathbb{C} \times \mathbb{Z} \times \mathbb{Z} / 2 \mathbb{Z}, \quad t_{s}(\nu, m, \delta)=(-\nu, m, m+\delta) .
$$


The $t_{s}$-fixed weights are

$$
\Lambda_{\text {fin }}^{t_{s}}\left(G, H_{s}\right)=\{(0, m, \delta) \mid m \in 2 \mathbb{Z}\} .
$$

The map of the proposition is

$$
I\left(R_{s}^{+}, R_{1}^{+}\right)(\nu, m, \delta)=(\nu, m) .
$$

This is indeed surjective (it is exactly two to one), and it intertwines the actions of $t_{s}$ and $t_{1}$; but it does not carry $t_{s}$-fixed weights onto $t_{1}$-fixed weights.

If for example we take $m=1$, we see that the character $r e^{i \phi} \mapsto e^{i \phi}$ of $H$, which is fixed by $t_{1}$, is not the highest weight of any $\theta$-fixed finitedimensional representation of $G$. It is actually the highest weight of two different representations of $G$, namely

$$
\mathbb{C}^{2} \otimes|\operatorname{det}|^{-1 / 2}, \quad \mathbb{C}^{2} \otimes|\operatorname{det}|^{-1 / 2} \otimes(\operatorname{sgn}(\operatorname{det})) .
$$

These two representations are interchanged by $\theta$ (since they are Hermitian dual to each other).

In order to avoid difficulties like those appearing in this example, we will use for extended groups only coherent families based on a maximally split Cartan. The definition makes sense more generally, however.

Definition 17.4 ([38], Definition 7.2.5). Suppose we are in the setting of (17.1), and $\lambda \in \mathfrak{h}^{*, t_{1}}$ is any $t_{1}$-fixed weight. Write

$$
\lambda+\Lambda_{\text {fin }}\left({ }^{\delta} G,{ }^{1} H\right)=\left\{\lambda+\psi \mid \psi \in \Lambda_{\text {fin }}\left({ }^{\delta} G,{ }^{1} H\right)\right\},
$$

(a set of formal symbols), called the translate of $\Lambda_{f i n}\left({ }^{\delta} G,{ }^{1} H\right)$ by $\lambda$. Similarly, we write

$$
\lambda+\mathcal{G}_{\text {fin }}\left({ }^{1} H\right)=\left\{\lambda+\psi \mid \psi \in \mathcal{G}_{\text {fin }}\left({ }^{1} H\right)\right\},
$$

(notation as in (17.1j) - another set of formal symbols, but on which we keep the abelian group structure from the Grothendieck group) called the translate of $\mathcal{G}_{\text {fin }}\left({ }^{1} H\right)$ by $\lambda$. An coherent family of virtual $\left(\mathfrak{g},{ }^{\delta} K\right)$ modules based on $\lambda+\Lambda_{\text {fin }}\left({ }^{\delta} G,{ }^{1} H\right)$ is a map

$$
\Phi_{1}: \lambda+\Lambda_{\text {fin }}\left({ }^{\delta} G,{ }^{1} H\right) \rightarrow \mathcal{G}\left(\mathfrak{g},{ }^{\delta} K\right)
$$

(notation as in Proposition 15.8) satisfying two conditions we will write below. Since the irreducible modules are a $\mathbb{Z}$-basis of the Grothendieck group, it is equivalent to give a $\mathbb{Z}$-linear map

$$
\Phi_{1}: \lambda+\mathcal{G}_{\text {fin }}\left({ }^{1} H\right) \rightarrow \mathcal{G}\left(\mathfrak{g},{ }^{\delta} K\right) .
$$

Here are the conditions. 
a) $\Phi_{1}(\lambda+\psi)$ has infinitesimal character $\lambda+d \psi \in \mathfrak{h}^{*}$; and

b) for every finite-dimensional algebraic representation $F_{1}$ of ${ }^{\delta} G$,

$$
F_{1} \otimes \Phi_{1}\left(\lambda+\psi_{1}\right)=\sum_{\mu_{1} \in \Delta\left(F_{1},{ }^{1} H\right)} \Phi_{1}\left(\lambda+\left[F \otimes \psi_{1}\right]\right)
$$

In (a), if $\psi$ is two-dimensional, then the statement means that each irreducible constituent of the restriction to $G$ of this virtual representation has infinitesimal character given by one of the two summands of the Lie algebra representation $\lambda+d \psi$. The brackets in (b) denote the class in $\mathcal{G}_{\text {fin }}\left({ }^{1} H\right)$, and we are using there the second formulation (17.4d) of the notion of coherent family.

The reader should verify that this formulation in the case of $G$ is precisely equivalent to the classical formulation in Definition 16.3. The language here avoids a tiresome enumeration of the various possible decompositions of tensor products of irreducible representations of ${ }^{1} H$.

There is an easy construction of some coherent families for ${ }^{\delta} G$.

Example 17.5. In the setting of Definition 17.4, suppose $\Phi$ is a coherent $\{$ ex: indcoh $\}$ family based on $\lambda+\Lambda_{\text {fin }}(G, H)$. Extend $\Phi$ to a $\mathbb{Z}$-linear map

$$
\Phi: \lambda+\mathcal{G}_{\text {fin }}(H) \rightarrow \mathcal{G}(\mathfrak{g}, K)
$$

as in $(17.4 \mathrm{~d})$. Define

$$
\Phi_{\text {ind }}\left(\lambda+\psi_{1}\right)=\operatorname{Ind}_{(\mathfrak{g}, K)}^{(\mathfrak{g}, \delta)}\left(\Phi\left(\lambda+\left.\psi_{1}\right|_{H}\right)\right) .
$$

That is, we restrict $\psi_{1}$ to $H$, getting one or two characters of $H$; we add the values of the coherent family for $G$ at those characters; then we induce from $G$ to ${ }^{\delta} G$.

That this construction gives a coherent family for ${ }^{\delta} G$ is elementary. The reason it is not so interesting is this. We saw in Theorem 16.6 that interesting information is encoded by a coherent family many of whose values are irreducible. The values of the coherent family $\Phi_{\text {ind }}$ are all induced; so the values can never include the type one irreducible modules for ${ }^{\delta} G$ (Definition 8.14), arising by extension of $\theta$-fixed irreducible modules for $G$.

Here is a description of more interesting coherent families. 
Theorem 17.6. Suppose $\Gamma_{1}=\left({ }^{1} H, \gamma_{1}, R_{i \mathbb{R}}^{+}\right)$is a Langlands parameter for $\{$ thm: extcohfam an irreducible representation $J\left(\Gamma_{1}\right)$ of ${ }^{\delta} G$, with $t_{1}$-fixed differential

$$
d \gamma_{1}=\left(d \gamma_{1}\right)^{t_{1}} \in \mathfrak{h}^{*}
$$

representing the infinitesimal character of $J\left(\Gamma_{1}\right)$. Just as in Theorem 16.6, we write $R\left(d \gamma_{1}\right)$ for the set of integral roots (Definition 16.4), and fix a set $R^{+}\left(d \gamma_{1}\right)$ of positive integral roots, subject to the requirements:

a) if $d \gamma_{1}\left(\alpha^{\vee}\right)$ is a positive integer, then $\alpha \in R^{+}\left(d \gamma_{1}\right)$;

b) if $d \gamma_{1}\left(\alpha^{\vee}\right)=0$, but $d \gamma_{1}\left(\theta \alpha^{\vee}\right)>0$, then $\alpha \in R^{+}\left(d \gamma_{1}\right)$;

c) $R^{+}\left(d \gamma_{1}\right) \supset R_{i \mathbb{R}}^{+}$; and

d) $t_{1}\left(R^{+}\left(d \gamma_{1}\right)\right)=R^{+}\left(d \gamma_{1}\right)$.

(Such a system always exists.) Fix a set $R^{+}=t_{1}\left(R^{+}\right)$of positive roots for $H$ in $G$ containing $R^{+}\left(d \gamma_{1}\right)$. Suppose finally that $H_{s}$ is a maximally split Cartan, with any positive root system $R_{s}^{+}$, as in Proposition 16.2; we will use the maps $i\left(R_{s}^{+}, R^{+}\right)$and $I\left(R_{s}^{+}, R^{+}\right)$defined in that proposition. Set

$$
d \gamma_{1, s}=i\left(R_{s}^{+}, R^{+}\right)^{-1}\left(d \gamma_{1}\right) \in \mathfrak{h}_{s}^{*}, \quad R^{+}\left(d \gamma_{1, s}\right)=i\left(R_{s}^{+}, R^{+}\right)^{-1}\left(R^{+}\left(d \gamma_{1}\right)\right) .
$$

Then there is a unique coherent family $\Phi_{1}$ of virtual $(\mathfrak{g}, K)$-modules based on $d \gamma_{1, s}+\Lambda_{f i n}\left(G,{ }^{1} H_{s}\right)$, with the following characteristic properties:

a) $\Phi_{1}\left(d \gamma_{1, s}\right)=\left[J\left(\Gamma_{1}\right)\right]$; and

b) whenever

i) $d \gamma_{1, s}+d \psi_{s}$ is integrally dominant (with respect to $R^{+}\left(d \gamma_{1, s}\right)$ ), and

ii) $\Gamma_{1}$ is type one, and

iii) $\psi_{s}$ is type one,

then $\Phi_{1}\left(d \gamma_{1, s}+\psi_{s}\right)$ is (the class in the Grothendieck group of) an irreducible representation or zero.

Let $\Gamma$ be an irreducible constituent of $\left.\Gamma_{1}\right|_{H}$ (of which there are one or two, depending on the type of $\left.\Gamma_{1}\right)$. Write $\Phi$ for the coherent family for $G$ based on $d \gamma_{1, s}+\Lambda_{f i n}\left(G, H_{s}\right)$ constructed in Theorem 16.6. 
1. If $\Gamma_{1}=\Gamma_{\text {ind }}$ is type two (equivalently, if $\Gamma$ is not fixed by $t_{1}$ ) then $\Psi_{1}=\Psi_{\text {ind }}$ (Example 17.5). In particular,

$$
\left.\Phi_{1}\left(d \gamma_{1, s}+\psi_{1}\right)\right|_{G}=\sum_{\psi \subset \psi_{1} \mid H} \Phi\left(d \gamma_{1, s}+\psi\right)+\Phi\left(d \gamma_{1, s}+\psi\right)^{t_{1}}
$$

Henceforth assume that $\Gamma_{1}$ is type one; equivalently, that $\Gamma$ is fixed by $t_{1}$. Write $\Pi\left(d \gamma_{1}\right)$ for the set of simple roots of $R^{+}\left(d \gamma_{1}\right)$, and define the $\tau$-invariant of $\Gamma_{1} \tau_{s}\left(\Gamma_{1}\right)={ }_{\text {def }} \tau_{s}(\Gamma) \subset \Pi_{s}\left(d \gamma_{1}\right)$ as in Theorem 16.6.

2.

$$
\left.\Phi_{1}\left(d \gamma_{1, s}+\psi_{1}\right)\right|_{G}=\sum_{\psi \subset \psi_{1} \mid H} \Phi\left(d \gamma_{1, s}+\psi\right)
$$

3. The $\tau$-invariant is preserved by $t_{s}$.

4. If $d \gamma_{s}+d \psi_{s}$ is integrally dominant, then $\Phi\left(d \gamma_{s}+\psi_{s}\right)=0$ if and only if there is a simple root $\alpha_{s} \in \tau_{s}(\Gamma)$ with $(d \gamma+d \psi)\left(\alpha_{s}^{\vee}\right)=0$.

5. Suppose that $d \gamma_{s}+d \psi_{s}$ is integrally dominant, and does not vanish on any root in $\tau_{s}\left(\Gamma_{1}\right)$. Write

$$
\psi=I\left(R_{s}^{+}, R^{+}\right)\left(\psi_{s}\right) \in \Lambda_{f i n}\left({ }^{1} H\right) .
$$

Then

$$
\Gamma_{1}+\psi={ }_{\operatorname{def}}\left({ }^{1} H, \gamma+\psi, R_{i \mathbb{R}}^{+}\right)
$$

is a Langlands parameter; and

$$
\Phi\left(d \gamma_{s}+\psi_{s}\right)=[J(\Gamma+\psi)]
$$

6. The set of irreducible constituents of the various virtual representations $\Psi_{1}\left(d \gamma_{s}+\psi_{s}\right)$ is equal to the set of irreducible constituents of all the tensor products $F_{1} \otimes J\left(\Gamma_{1}\right)$, with $F_{1}$ a finite-dimensional representation of ${ }^{\delta} G$.

Proof. The first issue is the existence of a $t_{1}$-stable set of positive integral roots. Recall from $(17.1)$ the $t_{1}$-stable set of positive roots $R_{1}^{+}$containing $R_{i \mathbb{R}}^{+}$. Using these, we can construct the desired set of positive integral roots by

$$
\begin{aligned}
& R^{+}\left(d \gamma_{1}\right)=\left\{\alpha \in R(G, H) \mid d \gamma_{1}\left(\alpha^{\vee}\right) \in \mathbb{Z}^{>0},\right. \text { or } \\
& \qquad \gamma_{1}\left(\alpha^{\vee}\right)=0, \text { and } d \gamma_{1}\left(\theta \alpha^{\vee}\right)>0, \text { or } \\
& \left.d \gamma_{1}\left(\alpha^{\vee}\right)=d \gamma_{1}\left(\theta \alpha^{\vee}\right)=0, \text { and } \alpha \in R_{1}^{+}\right\} .
\end{aligned}
$$


Conditions (a) and (b) from the theorem are obviously satisfied. Condition (c) follows from the requirement that $d \gamma_{1}$ is weakly dominant for $R_{i \mathbb{R}}^{+}$(Theorem 6.1), and the hypothesis that $R_{i \mathbb{R}}^{+} \subset R_{1}^{+}$(Definition 13.7. For (d), since $t_{1}$ fixes both $d \gamma_{1}$ and $R_{1}^{+}$, and $t_{1}$ commutes with the action of $\theta$ on $H$, it follows that $t_{1}$ preserves $R^{+}\left(d \gamma_{1}\right)$.

For the coherent family, suppose first that $\Gamma_{1}=\Gamma_{\text {ind }}$ is of type two, so that

$$
J\left(\Gamma_{1}\right)=J\left(\Gamma_{\text {ind }}\right)=J(\Gamma)_{\text {ind }} .
$$

Let $\Phi$ be the coherent family for $J(\Gamma)$ based on $d \gamma_{s}+\Lambda_{\text {fin }}\left(G, H_{s}\right)$ (Theorem 16.6), and form the induced coherent family $\Phi_{1}=\Phi_{\text {ind }}$ as in Example 17.5. Then the characteristic properties (a) and (b) are immediate, assertion (1) is true by definition, and the rest of the theorem does not apply.

Next suppose that $\Gamma_{1}$ is type one, so that it is one-dimensional and extends the Langlands parameter $\Gamma$ for $G$. There are two of these extensions $\Gamma_{+}$and $\Gamma_{-}$, to each of which we would like to attach a coherent family $\Phi_{ \pm}$. What is easy is that

$$
\Phi_{+}+\Phi_{-}=\Phi_{\text {ind }},
$$

in the sense that the induced coherent family behaves as the theorem says that the sum ought to; what is harder is to find the two summands individually. For this we return to the proof of Theorem 16.6 in [38]. Because parabolic and cohomological induction behave well with respect to translation functors, we can use either Definition 13.14 or Definition 13.15 to construct a coherent family $\Phi_{1}^{\text {std }}$ for ${ }^{\delta} G$ (based on ${ }^{1} H$ ) satisfying

$$
\Phi_{1}^{\mathrm{std}}\left(d \gamma_{1}\right)=I_{\mathrm{quo}}\left(\Gamma_{1}\right) .
$$

Using these building blocks (and taking $\mathbb{Z}$-linear combinations) we can follow the argument for Corollary 7.3.23 in [38] to construct the coherent family $\Phi_{1}$ that we seek.

The construction makes clear the description in (2) of the restriction to $G$. The statement in (3) means that the corresponding set of simple roots for $R^{+}\left(d \gamma_{1}\right)$ is preserved by $t_{1}$. This is obvious from the description of $\tau$ in Theorem 16.6. The statement in (4) about vanishing reduces at once to $G$, where it is part of Theorem 16.6. The calculation in (5) of Langlands parameters follows from the construction sketched above for $\Phi_{1}$. Part (6) is a formal consequence of the definition of coherent family.

In order to discuss translation functors for extended groups, we need $\{$ se: exttransfunc\} the language of (15.1) and (16.8) explicitly extended to ${ }^{\delta} G$. So we fix a 
maximally split Cartan and a minimal parabolic subgroup

$$
H_{s}=T_{s} A_{s}, \quad M_{s}=K^{A_{s}}, \quad P_{s}=M_{s} A_{s} N_{s}
$$

of $G$, and a system of positive imaginary roots $R_{s, i \mathbb{R}}^{+}$for $M$. These define a positive system

$$
R_{s}^{+}=R_{s, i \mathbb{R}}^{+} \cup\left(\text { roots of } H_{s} \text { in } N_{s}\right)=R_{s, i \mathbb{R}}^{+} \cup R\left(N_{s}, H_{s}\right)
$$

for $G$. Define a Weyl group element $w_{s}$ by the requirement

$$
\theta\left(R_{s}^{+}\right)=R_{s, i \mathbb{R}}^{+} \cup-R\left(N_{s}, H_{s}\right)=w_{s}^{-1}\left(R_{s}^{+}\right) .
$$

This is the long element of the "little Weyl group" of $A_{s}$ in $G$, so in particular it belongs to $W\left(G, H_{s}\right)$. Setting

$$
t_{s}=w_{s} \theta_{H_{s}}, \quad t_{s}\left(R_{s}^{+}\right)=R_{s}^{+}
$$

we find that a representative $\delta_{s}$ for $t_{s}$ generates with $H_{s}$ an extended torus ${ }^{1} H_{s}$.

Fix also a weight

$$
\lambda_{0} \in \mathfrak{h}_{s}^{*}
$$

corresponding to a (possibly singular) infinitesimal character

$$
\xi_{\lambda_{0}}: \mathfrak{Z}(\mathfrak{g}) \rightarrow \mathbb{C}
$$

To simplify the discussion slightly (although it is not necessary) we assume that $\lambda_{0}$ is real ([38], Definition 5.4.11). We need (for the abstract theory of translation functors) to assume that $\lambda_{0}$ is integrally dominant for $R_{s}^{+}$; and we assume also that $\lambda_{0}$ is real dominant $((16.4 \mathrm{~d}))$ :

$$
\lambda_{0}\left(\alpha^{\vee}\right) \geq 0, \quad\left(\alpha \in R_{s}^{+}\right) .
$$

$\{$ eq: sdominant $\}$

This requirement determines $\lambda_{0}$ uniquely in its Weyl group orbit. Because $t_{s}$ preserves $R_{s}^{+}$, the weight $t_{s}\left(\lambda_{0}\right)$ shares the dominance property $(17.8 \mathrm{~g})$. As a consequence,

$$
\begin{aligned}
t_{s}\left(\lambda_{0}\right)=\lambda_{0} & \Longleftrightarrow t_{s}\left(\lambda_{0}\right) \in W \cdot \lambda_{0} \\
& \Longleftrightarrow \text { infinitesimal character } \xi_{\lambda_{0}} \text { fixed by } \theta
\end{aligned}
$$

Define

$$
\begin{gathered}
{ }^{\delta} B\left(\lambda_{0}\right)=\text { equivalence classes of extended Langlands parameters } \\
\text { of infinitesimal character } \lambda_{0} ;
\end{gathered}
$$


as usual for the extended group, "infinitesimal character $\lambda_{0}$ " means "annihilated by $\left[\operatorname{ker} \xi_{\lambda_{0}}\right]\left[\operatorname{ker} \xi_{t_{s} \lambda_{0}}\right] . "$

If $\lambda_{0} \neq t_{s}\left(\lambda_{0}\right)$, then by (17.8h) the infinitesimal character $\xi_{\lambda_{0}}$ is not fixed by $\theta$, so no representation of $G$ of infinitesimal character $\xi_{\lambda_{0}}$ is fixed by $\theta$. By Clifford theory (Proposition 8.13), in this case all representations of infinitesimal character $\lambda_{0}$ are induced from $G$. Once all the representations are induced, then any question about character theory can be reduced at once to $G$. (We will recall in detail how this works in Section 19.)

We therefore only need to analyze the case

$$
t_{s}\left(\lambda_{0}\right)=\lambda_{0}
$$

Write $R\left(\lambda_{0}\right)$ for the integral roots (Definition 16.4), and write

$$
R^{+}\left(\lambda_{0}\right)=_{\text {def }} R\left(\lambda_{0}\right) \cap R_{s}^{+},
$$

which we have assumed makes $\lambda_{0}$ integrally dominant. Write

$$
\Pi\left(\lambda_{0}\right) \subset R^{+}\left(\lambda_{0}\right)
$$

and

$$
\left\{\alpha \in \Pi\left(\lambda_{0}\right) \mid \lambda_{0}\left(\alpha^{\vee}\right)=0\right\}={ }_{\text {def }} \Pi^{\lambda_{0}}
$$

for the subset of simple roots orthogonal to $\lambda_{0}$. Since $t_{s}\left(\lambda_{0}\right)=\lambda_{0}$, the sets $\Pi^{\lambda_{0}} \subset \Pi\left(\lambda_{0}\right)$ are preserved by $t_{s}$. If $X_{1} \in{ }^{\delta} B\left(\lambda_{0}\right)$ is a type one irreducible, extending a type one irreducible $X$ for $G$, we have defined the $\tau$-invariant

$$
\tau\left(X_{1}\right)=\tau(X) \subset \Pi\left(\lambda_{0}\right)
$$

$\{$ eq: type1tau $\}$

in Theorem 17.6. If $Y_{1}=Y_{\text {ind }}$ is type two, then we define

$$
\tau\left(Y_{\text {ind }}\right)=_{\text {def }}\left(\tau(Y), \tau\left(Y^{t_{s}}\right)\right)=\left(\tau(Y), t_{s}(\tau(Y)),\right.
$$

$\{$ eq: type2tau

an unordered pair of subsets of $\Pi\left(\lambda_{0}\right)$. (In order to unify the notation, we may sometimes regard $\tau\left(X_{1}\right)$ as the unordered pair $(\tau(X), \tau(X))$.)

Finally, we fix a type one weight

$$
\phi \in \Lambda_{\mathrm{fin}}\left(G, H_{s}\right)^{t_{s}}
$$

of a finite-dimensional representation of $G$, dominant for $R_{s}^{+}$, and such that

$$
\lambda_{0}+d \phi \text { is dominant and regular for } R^{+}\left(\lambda_{0}\right) .
$$


Just as in (16.8h), for the purpose of studying signatures we will want to assume in addition

$$
\lambda_{0}+d \phi \text { is dominant and regular for } R_{s}^{+} .
$$

$\{$ eq:extrealdom $\}$

(One way to achieve this is to take $\phi$ to be the sum of the roots in $R_{s}^{+}$.) Write

$$
\phi_{ \pm} \in \Lambda_{\mathrm{fin}}\left({ }^{\delta} G,{ }^{1} H_{s}\right)
$$

for the two extensions of $\phi$ to ${ }^{1} H_{s}$. Now we can write

$$
F_{\phi_{ \pm}}=\text {finite-dimensional irreducible for }{ }^{\delta} G \text { of highest weight } \phi_{ \pm} .
$$

In order to define the Jantzen-Zuckerman translation functors, we need the functor (on $\mathfrak{g}$-modules)

$$
P_{\lambda_{0}}(M)=\begin{aligned}
& \text { largest submodule of } M \text { on which } z-\xi_{\lambda_{0}}(z) \\
& \text { acts nilpotently, all } z \in \mathfrak{Z}(\mathfrak{g})
\end{aligned}
$$

If $M$ is a $\left(\mathfrak{g},{ }^{\delta} K\right)$ module, so is $P_{\lambda_{0}}(M)$. On the category of $\mathfrak{Z}(\mathfrak{g})$-finite modules (for example, on $\left(\mathfrak{g},{ }^{\delta} K\right)$-modules of finite length) the functor $P_{\lambda_{0}}$ is exact. The translation functors we want are

$$
T_{\lambda_{0}}^{\lambda_{0}+\phi_{+}}(M)={ }_{\text {def }} P_{\lambda_{0}+d \phi}\left(F_{\phi_{+}} \otimes P_{\lambda_{0}}(M)\right),
$$

$\{$ eq:exttransfunc $\}$

("translation away from the wall") and

$$
T_{\lambda_{0}+\phi_{+}}^{\lambda_{0}}(N)={ }_{\text {def }} P_{\lambda_{0}}\left(F_{\phi_{+}}^{*} \otimes P_{\lambda_{0}+d \phi}(N)\right)
$$

("translation to the wall.") These are clearly exact functors on the category of finite-length $\left(\mathfrak{g},{ }^{\delta} K\right)$-modules, so they also define group endomorphisms of the Grothendieck group $\mathcal{G}\left(\mathfrak{g},{ }^{\delta} K\right)$.

These translation functors are quite special, in the sense that both the domain and the range infinitesimal characters are assumed to be represented by a $t_{s}$-fixed weight. There are some reasonable statements to be made in more generality; but most of those statements concern type two representations, which are induced from $G$; so they can be deduced easily from (for example) Corollary 16.9. We are concerned here only with the more delicate situation for type one representations.

Corollary 17.9 (Jantzen [16], Zuckerman [47]; see [38], Proposition 7.2.22). \{cor: exttransfunc\} Suppose we are in the setting of (17.8), so that in particular $\xi_{\lambda_{0}}$ (possibly singular) and $\xi_{\lambda_{0}+\phi}$ (regular) are infinitesimal characters fixed by twisting by $\theta$. 
1. The functors $T_{\lambda_{0}+\phi_{+}}^{\lambda_{0}}$ and $T_{\lambda_{0}}^{\lambda_{0}+\phi_{+}}$are left and right adjoint to each other:

$$
\operatorname{Hom}_{\mathfrak{g}, \delta}\left(N, T_{\lambda_{0}}^{\lambda_{0}+\phi_{+}}(M)\right) \simeq \operatorname{Hom}_{\mathfrak{g},{ }^{\prime} K}\left(T_{\lambda_{0}+\phi_{+}}^{\lambda_{0}}(N), M\right),
$$

and similarly with the two functors interchanged.

2. If $\Phi_{1}$ is a coherent family (Definition 17.4) on $\lambda_{0}+\Lambda_{\text {fin }}\left(G,{ }^{1} H_{s}\right)$,

$$
T_{\lambda_{0}+\phi_{+}}^{\lambda_{0}}\left(\Phi_{1}\left(\lambda_{0}+\phi_{+}\right)\right)=\Phi_{1}\left(\lambda_{0}\right) \text {. }
$$

3. The translation functor $T_{\lambda_{0}+\phi_{+}}^{\lambda_{0}}$ (translation to the wall) carries every irreducible $\left(\mathfrak{g},{ }^{\delta} K\right)$-module of infinitesimal character $\lambda_{0}+d \phi$ to an irreducible of infinitesimal character $\lambda_{0}$, or to zero. The irreducible representations mapped to zero are exactly those containing some root of $\Pi^{\lambda_{0}}$ in the $\tau$-invariant (Definition 16.7, (17.80)).

4. The (inverse of the) translation functor of (3) defines an injective correspondence of Langlands parameters (and therefore of irreducible representations)

$$
t_{\lambda_{0}}^{\lambda_{0}+\phi_{+}}:{ }^{\delta} B\left(\lambda_{0}\right) \hookrightarrow{ }^{\delta} B\left(\lambda_{0}+d \phi\right) .
$$

The image consists of all irreducible representations (of infinitesimal character $\lambda_{0}+d \phi$ ) having no root of $\Pi^{\lambda_{0}}$ in the $\tau$-invariant.

5. Suppose $\Gamma_{2}=\left({ }^{2} H, \gamma_{2}, R_{i \mathbb{R}}^{+}\right) \in{ }^{\delta} B\left(\lambda_{0}\right)$ is a Langlands parameter (at the possibly singular infinitesimal character $\lambda_{0}$ ) for an irreducible representation $J\left(\Gamma_{2}\right)$ of ${ }^{\delta} G$. Choose positive integral roots $R^{+}\left(d \gamma_{2}\right)$ as in Theorem 17.6, so that we have an isomorphism

$$
i\left(\lambda_{0}, R^{+}\left(\lambda_{0}\right), d \gamma_{2}, R^{+}\left(d \gamma_{2}\right)\right): \mathfrak{h}_{s}^{*} \rightarrow \mathfrak{h}^{*}
$$

as in (16.5), and a map of virtual representations

$$
{ }^{\delta} I\left(\lambda_{0}, R^{+}\left(\lambda_{0}\right), d \gamma_{2}, R^{+}\left(d \gamma_{2}\right)\right): \mathcal{G}_{f i n}\left({ }^{1} H_{s}\right) \rightarrow \mathcal{G}_{f i n}\left({ }^{1} H\right)
$$

as in Proposition 17.2(2). Define

$$
\phi\left(\Gamma_{2}\right)_{+}={ }^{\delta} I\left(\lambda_{0}, R^{+}\left(\lambda_{0}\right), d \gamma_{2}, R^{+}\left(d \gamma_{2}\right)\right)\left(\phi_{+}\right),
$$

a (type one) ${ }^{2} H$-weight of a finite-dimensional representation of ${ }^{\delta} G$. (Proposition 17.2). Then the correspondence of Langlands parameters in (4) is

$$
t_{\lambda_{0}}^{\lambda_{0}+\phi_{+}}\left(\Gamma_{2}\right)=\Gamma_{2}+\phi\left(\Gamma_{2}\right)_{+}
$$

(notation as in Theorem 17.6(4)). 
6. Suppose $\Gamma_{1}=\left(H, \gamma_{1}, R_{i \mathbb{R}}^{+}\right) \in{ }^{\delta} B\left(\lambda_{0}+d \phi\right)$ is a Langlands parameter (at the regular infinitesimal character $\left.\lambda_{0}+d \phi\right)$. Write $R^{+}\left(d \gamma_{1}\right)$ for the unique set of positive integral roots making $d \gamma_{1}$ integrally dominant, so that we have an isomorphism

$$
i\left(\lambda_{0}+d \phi, R^{+}\left(\lambda_{0}\right), d \gamma_{1}, R^{+}\left(d \gamma_{1}\right)\right): \mathfrak{h}_{s}^{*} \rightarrow \mathfrak{h}^{*}
$$

as in (16.5). Define

$$
\phi\left(\Gamma_{1}\right)_{+}={ }^{\delta} I\left(\lambda_{0}, R^{+}\left(\lambda_{0}\right), d \gamma_{0}, R^{+}\left(d \gamma_{0}\right)\right)\left(\phi_{+}\right),
$$

a (type one) ${ }^{2} \mathrm{H}$-weight of a finite-dimensional representation of $G$ (Proposition 16.2). Then

$$
t_{\lambda_{0}+\phi_{+}}^{\lambda_{0}}\left(\Gamma_{1}\right)=_{\text {def }}\left(H, \gamma_{1}-\phi\left(\Gamma_{1}\right)_{+}, R_{i \mathbb{R}}^{+}\right)=_{\text {def }} \Gamma_{1}-\phi\left(\Gamma_{1}\right)_{+}
$$

is a weak extended Langlands parameter (Definition 13.7). This correspondence is a left inverse to the injection of (4):

$$
t_{\lambda_{0}+\phi_{+}}^{\lambda_{0}} \circ t_{\lambda_{0}}^{\lambda_{0}+\phi_{+}}\left(\Gamma_{0}\right)=\Gamma_{0}
$$

7. Translation to the wall carries standard representations to weak standard representations:

$$
T_{\lambda_{0}+\phi_{+}}^{\lambda_{0}}\left(I_{\text {quo }}\left(\Gamma_{1}\right)=I_{\text {quo }}\left(\Gamma_{1}-\phi\left(\Gamma_{1}\right)_{+}\right) .\right.
$$

In particular,

$$
T_{\lambda_{0}+\phi_{+}}^{\lambda_{0}}\left(I_{\mathrm{quo}}\left(\Gamma_{0}+\phi\left(\Gamma_{0}\right)_{+}\right)=I_{\mathrm{quo}}\left(\Gamma_{0}\right)\right.
$$

8. Translation to the wall carries irreducibles to irreducibles or to zero:

$$
T_{\lambda_{0}+\phi_{+}}^{\lambda_{0}}\left(J\left(\Gamma_{1}\right)= \begin{cases}J\left(\Gamma_{1}-\phi\left(\Gamma_{1}\right)_{+}\right) & \left(\tau\left(J\left(\Gamma_{1}\right)\right) \cap \Pi^{\lambda_{0}}=\emptyset\right), \\ 0 & \left(\tau\left(J\left(\Gamma_{1}\right)\right) \cap \Pi^{\lambda_{0}} \neq \emptyset\right) .\end{cases}\right.
$$

In particular,

$$
T_{\lambda_{0}+\phi_{+}}^{\lambda_{0}}\left(J\left(\Gamma_{0}+\phi\left(\Gamma_{0}\right)_{+}\right)=J\left(\Gamma_{0}\right) .\right.
$$

Corollary 17.9 allows us to "reduce" the computation of the multiplicity \{se: extreducetoreg\} matrix $m_{\Xi, \Gamma}$ defined in (15.1) to the case of regular infinitesimal character. Given a possibly singular infinitesimal character $\lambda_{0}$, we choose a type one weight of a finite-dimensional representation $\phi_{+}$as in (17.8), so that $\lambda_{0}+d \phi$ is regular. The (possibly singular) Langlands parameters $\Xi$ and $\Gamma$ in ${ }^{\delta} B\left(\lambda_{0}\right)$ 
correspond to regular Langlands parameters $\Xi+\phi(\Xi)_{+}$and $\Gamma+\phi(\Gamma)_{+}$in ${ }^{\delta} B\left(\lambda_{0}+d \phi\right)$, and

$$
m_{\Xi, \Gamma}=m_{\Xi+\phi(\Xi)_{+}, \Gamma+\phi(\Gamma)_{+}} \cdot
$$

A little more precisely, we can start with the decomposition into irreducibles

$$
\left[I\left(\Gamma+\phi(\Gamma)_{+}\right)\right]=\sum_{\Xi_{1} \in \delta} m_{\left.\Xi_{1}, \Gamma+\phi(\Gamma)_{+}+d \phi\right)}\left[J\left(\Xi_{1}\right)\right]
$$

and apply the exact translation functor $T_{\lambda_{0}+\phi}^{\lambda_{0}}$. On the left we get $[I(\Gamma)]$, by Corollary 17.9(7). On the right we get

$$
\sum_{\substack{\Xi_{1} \in B\left(\lambda_{0}+d \phi\right) \\ \tau\left(\Xi_{1}\right) \cap \Pi^{\lambda_{0}}=\emptyset}} m_{\Xi_{1}, \Gamma+\phi(\Gamma)_{+}}\left[J\left(\Xi_{1}-\phi\left(\Xi_{1}\right)_{+}\right)\right] .
$$

The irreducibles appearing on the right are all distinct (this is part of the uniqueness assertion for the coherent family in Theorem 16.6), so the coefficients are the multiplicities of irreducibles in $I(\Gamma)$.

To say this another way: the index set of extended Langlands parameters at the possibly singular infinitesimal character $\lambda_{0}$ is naturally a subset of the index set at the regular infinitesimal character $\lambda_{0}+d \phi$. The multiplicity matrix at $\lambda_{0}$ is the corresponding submatrix of the one at $\lambda_{0}+d \phi$.

Finally, we record the reduction to regular infinitesimal character of the character matrix $M_{\Xi, \Gamma}$.

Theorem 17.11. Suppose we are in the setting of (17.8); in particular, we assume that the infinitesimal characters $\lambda_{0}$ and $\lambda_{0}+d \phi$ are both fixed by $\theta$. Write

$$
t_{\lambda_{0}+\phi_{+}}^{\lambda_{0}}:{ }^{\delta} B\left(\lambda_{0}+d \phi\right) \rightarrow{ }^{\delta} B_{\text {weak }}\left(\lambda_{0}\right)
$$

(notation (15.1)) for the map of Corollary 17.9(6). Fix an extended Langlands parameter $\Xi_{1} \in{ }^{\delta} B\left(\lambda_{0}+d \phi\right)$. Write

$$
\Xi_{0}=t_{\lambda_{0}+\phi_{+}}^{\lambda_{0}}\left(\Xi_{1}\right) \in{ }^{\delta} B_{\text {weak }}\left(\lambda_{0}\right)
$$

so that

$$
T_{\lambda_{0}+\phi_{+}}^{\lambda_{0}}\left(I_{\text {quo }}\left(\Xi_{1}\right)\right)=I_{\text {quo }}\left(\Xi_{0}\right) .
$$

1. There is a unique subset $C_{\text {sing }}\left(\Xi_{0}\right) \subset{ }^{\delta} B\left(\lambda_{0}\right)$ with the property that

$$
I_{\mathrm{quo}}\left(\Xi_{0}\right)=\sum_{\Xi^{\prime} \in C_{\text {sing }}\left(\Xi_{0}\right)} I_{\mathrm{quo}}\left(\Xi^{\prime}\right)
$$


2. The set $C_{\text {sing }}\left(\Xi_{0}\right)$ has cardinality either equal to zero (in case some simple compact imaginary root for $\Xi_{0}$ vanishes on $\lambda_{0}$, so that $I\left(\Xi_{0}\right)=$ 0) or a power of two.

For each Langlands parameter $\Xi \in{ }^{\delta} B\left(\lambda_{0}\right)$, define

$$
C_{r e g}(\Xi)=\left\{\Xi_{1} \in{ }^{\delta} B\left(\lambda_{0}+d \phi\right) \mid \Xi \in C_{\text {sing }}\left(\Xi_{0}\right)\right\} \subset{ }^{\delta} B\left(\lambda_{0}+d \phi\right) ;
$$

here $\Xi_{0}=t_{\lambda_{0}+\phi_{+}}^{\lambda_{0}}\left(\Xi_{1}\right)$ as above.

3. Suppose $\Gamma \in{ }^{\delta} B\left(\lambda_{0}\right)$ is any Langlands parameter, corresponding to $\Gamma+\phi(\Gamma) \in{ }^{\delta} B\left(\lambda_{0}\right)$. Then the character formula (15.11) is

$$
J(\Gamma)=\sum_{\Xi \in \in^{\delta} B\left(\lambda_{0}\right)} \sum_{\Xi_{1} \in C_{r e g}(\Xi)} M_{\Xi_{1}, \Gamma+\phi(\Gamma)}[I(\Xi)] .
$$

Equivalently,

$$
M_{\Xi, \Gamma}=\sum_{\Xi_{1} \in C_{r e g}(\Xi)} M_{\Xi_{1}, \Gamma+\phi(\Gamma)} .
$$

The proof is identical to that of Theorem 16.11.

\section{KLV theory}

We have seen in (15.1) how to write down finite matrices over $\mathbb{Z}$

$$
\left(M_{\Gamma, \Psi}\right), \quad\left(m_{\Xi, \Gamma}\right) \quad(\Gamma, \Psi \in B(\chi))
$$

describing the characters of irreducible representations. In the present section we will recall how Kazhdan and Lusztig computed the character matrices. Carrying this out for the extended group ${ }^{\delta} G$ requires significant effort (most of which is carried out in [27]); we will therefore discuss that case separately in Section 19. However, a number of the preliminary definitions given here extend easily to the extended group case; we will try to note that explicitly as they arise.

A $q$-analogue of an integer $n$ is a polynomial $N \in \mathbb{Z}[q]$ with the property that $N(1)=n$. To be an interesting $q$-analogue, the coefficients of $N$ should carry refined information about some question to which $n$ is an answer.

The first step in the Kazhdan-Lusztig idea is to form $q$-analogues of the multiplicities and signatures. The matrices over $\mathbb{Z}$ are then replaced by matrices over $\mathbb{Z}[q]$. The polynomial entries satisfy recursion relations that are not visible at $q=1$, so they can be computed. 
Kazhdan and Lusztig in [17] introduced the $q$-analogues in a way that is quite subtle and complicated (transforming the problem first into algebraic geometry, then into finite characteristic, and finally studying eigenvalues of a Frobenius operator). The advantage of their approach was that Deligne and others ([4]) had proven deep facts about these eigenvalues. We use an approach that is much simpler to explain, but apparently much harder to prove anything about. Fortunately Beilinson and Bernstein in [3] have already proven the equivalence of the two approaches.

In [17], the definitions of $q$-analogues are mostly geometric, but with modifications by powers of $q$ given by lengths of Weyl group elements (which played the role of Langlands parameters). We need to make such modifications in our setting as well, so we need a notion of length for Langlands parameters.

Definition 18.2 ([38], Definition 8.1.4). Suppose $\Gamma=\left(H, \gamma, R_{i \mathbb{R}}^{+}\right)$is a $\{$ def $:$ length $\}$ Langlands parameter for $G$ (Theorem 6.1 ). Choose a system of positive integral roots $R^{+}(d \gamma)$ subject to the requirements in Theorem 16.6. Let $L_{\text {real }}$ be a split semisimple real group with root system the set $R_{\mathbb{R}}(d \gamma)$ of integral real roots, and $K_{\text {real }}$ a maximal compact subgroup of $L_{\text {real }}$. Define

$$
\begin{aligned}
c_{\text {real }} & ={ }_{\text {def }} \#\left\{\text { positive roots for } L_{\text {real }}\right\}-\#\left\{\text { positive roots for } K_{\text {real }}\right\} . \\
& =\text { dimension of a Borel subgroup of } K_{\text {real }}(\mathbb{C}) .
\end{aligned}
$$

(The reason for the equality is that the Iwasawa decomposition for the split group $L_{\text {real }}$ implies that

$$
\left.\operatorname{dim} K_{\text {real }}=\#\left\{\text { positive roots for } L_{\text {real }}\right\} .\right)
$$

The (integral) length of $\Gamma$ is

$$
\ell(\Gamma)=\#\left\{\text { pairs }(\alpha,-\theta(\alpha)) \text { of complex roots in } R^{+}(d \gamma)\right\}+c_{\text {real }} .
$$

Because this definition of length is natural, it is unchanged by twisting $\Gamma$ by any automorphism commuting with $\theta$. In particular, it extends immediately to parameters for the extended group: the length of a parameter for ${ }^{\delta} G$ is just the length of any constituent of its restriction to $G$.

Proposition 18.3 ([38], Proposition 8.6.19). Suppose that $\Xi$ and $\Gamma$ are \{prop:length\} Langlands parameters, and that $J(\Xi)$ occurs as a composition factor in the standard module $I(\Gamma)$. Then

$$
\ell(\Xi) \leq \ell(\Gamma)
$$


Equality holds if and only if $\Xi$ is equivalent to $\Gamma$ (so that $J(\Xi)$ is equal to the Langlands subquotient $J(\Gamma))$.

Exactly the same statement holds for the extended group ${ }^{\delta} G$.

This Proposition provides another proof of the upper triangularity of the multiplicity matrix proved in (15.1i):

Corollary 18.4. Suppose that $m_{\Xi, \Gamma} \neq 0$. Then

$$
\ell(\Xi) \leq \ell(\Gamma)
$$

Equality holds if and only if $\Xi$ is equivalent to $\Gamma$. Consequently the multiplicity matrix $m_{\Xi, \Gamma}$ is upper triangular with respect to any ordering of the Langlands parameters consistent with length.

This Corollary is all that we really need about the upper triangular nature of the multiplicity matrix; but having come so far, we will pause to include a more precise statement.

Definition 18.5. Suppose $H$ is a Cartan subgroup of $G$, and $\lambda_{1} \in \mathfrak{h}^{*}$ is a regular weight. Recall from (15.1) the finite set $B\left(\lambda_{1}\right)$ of (equivalence classes of) Langlands parameters of infinitesimal character $\lambda_{1}$. The Bruhat order on $B\left(\lambda_{1}\right)$ is the transitive closure of the relation

$$
\Xi<\Gamma \text { if } J(\Xi) \text { is a composition factor of } I(\Gamma) .
$$

This definition makes sense for ${ }^{\delta} G$.

(2) This definition makes perfectly good sense for singular infinitesimal II character, but we choose not to use it in that case. The reason is that the order with this definition is easy to compute (for $G$ ) in the case of regular infinitesimal character (see the remarks after Proposition 18.8 below), but would not be easy to compute for singular infinitesimal character. Instead we use

Definition 18.6. Suppose $H_{s}$ is a maximally split Cartan subgroup \{def:singbruhat $\}$ of $G$, and $\lambda_{0} \in \mathfrak{h}_{s}^{*}$ is a a possibly singular weight. Choose a weight $\phi \in \Lambda_{\mathrm{fin}}\left(G, H_{s}\right)$ as in (16.8), so that (among other things)

$$
\lambda_{1}=\lambda_{0}+d \phi
$$

is a regular weight, and there is an inclusion of Langlands parameters

$$
t_{\lambda_{0}}^{\lambda_{0}+\phi}: B\left(\lambda_{0}\right) \hookrightarrow B\left(\lambda_{1}\right)
$$

(Corollary 16.9). The Bruhat order on $B\left(\lambda_{0}\right)$ is the Bruhat order on $B\left(\lambda_{1}\right)$, pulled back by $t_{\lambda_{0}}^{\lambda_{0}+\phi}$. 
The preservation of multiplicities explained in (16.10) shows first of all that if $\lambda_{0}$ is regular, then this definition agrees with Definition 18.5 (even if we use a nonzero $\phi$ ). It also follows that

$$
J\left(\Xi_{0}\right) \text { a composition factor of } I\left(\Gamma_{0}\right) \Longrightarrow \Xi_{0} \leq \Gamma_{0} .
$$

What is different in the singular case is that there may be additional relations in the Bruhat order not generated by these "composition factor" relations.

Proposition 18.8. Fix a regular weight $\lambda_{1} \in \mathfrak{h}^{*}$. The Bruhat order on $B\left(\lambda_{1}\right)$ (Definition 18.5) is graded by the length function of Definition 18.2: if $\ell(\Gamma)=p$, then every immediate predecessor $\Xi$ of $\Gamma$ has $\ell(\Xi)=p-1$.

The statement and proof of this proposition carry over to the extended group ${ }^{\delta} G$ with little change. In the case of $G$, the proof also provides a recursion (on $\ell(\Gamma)$ ) for computing the Bruhat order; but this recursion does not carry over in a simple fashion to ${ }^{\delta} G$. A short version of the difficulty is that "complex cross actions for the extended group may be double-valued.") We do not need this computation, so we have not pursued the matter.

The recursive computation of the Bruhat order is implemented by the command blockorder in the software atlas written by Fokko du Cloux and Marc van Leeuwen ([7]).

Proof. This is nearly a consequence of the proof of Prop 8.6.19 of [38]. The argument given there for "Case I" (or in fact also for "Case II") serves perfectly as an inductive step in the proof of the stronger statement given in this proposition. The difficulty arises only in "Case III" of that proof. So we may assume that Case I does not arise for any simple root: that is, that

$$
\theta \alpha \in R^{+}(d \gamma), \quad(\alpha \in \Pi(d \gamma) \text { complex }) .
$$

According to [38], Lemma 8.6.1, this is equivalent to

$$
\left.\theta\left(R^{+}(d \gamma)\right)-R_{\mathbb{R}}^{+}(d \gamma)\right)=R^{+}(d \gamma)-R_{\mathbb{R}}^{+}(d \gamma):
$$

the Cartan involution $\theta$ preserves the set of non-real integral roots. Just as in (9.3), we define

$$
L=\text { centralizer in } G \text { of } T_{0} \text {, }
$$

the reductive subgroup generated by $H$ and its real roots. Define a Langlands parameter $\Gamma_{\mathfrak{q}}$ for $L$ as in (9.3); the corresponding standard representation $I\left(\Gamma_{\mathfrak{q}}\right)$ is an ordinary principal series representation of $L$. What is important here is that if $\Xi_{\mathfrak{q}}$ is any Langlands parameter for $L$ of infinitesimal 
character $d \gamma_{\mathfrak{q}}$, then Zuckerman's cohomological induction functor carries the irreducible for $L$ to the irreducible for $G$

$$
\left(\mathcal{L}_{\overline{\mathfrak{q}}, L \cap K}^{\mathfrak{g}, K}\right)^{s}\left(J\left(\Xi_{\mathfrak{q}}\right)\right)=J(\Xi)
$$

and correspondingly for standard modules

$$
\left(\mathcal{L}_{\overline{\mathfrak{q}}, L \cap K}^{\mathfrak{g}, K}\right)^{s}\left(I\left(\Xi_{\mathfrak{q}}\right)\right)=I(\Xi) .
$$

These facts show that the inclusion of Langlands parameters

$$
B_{L}\left(d \gamma_{\mathfrak{q}}\right) \hookrightarrow B_{G}(d \gamma), \quad \Xi_{\mathfrak{q}} \mapsto \Xi
$$

respects Bruhat orders, and in fact carries the Bruhat interval below $\Gamma_{\mathfrak{q}}$ isomorphically onto the Bruhat interval below $\Gamma$. It is easy to see that the inclusion preserves lengths.

So the inductive step at $\Gamma$ in the proof of our proposition may be reduced to the case $G=L$ : that is, to the case that $I(\Gamma)$ is an ordinary principal series representation for the split group $L$. We assume this for the rest of the proof. To simplify the notation, we will assume also that all the odd roots are "type II". (If this assumption fails and there is a "type I" root, then as remarked at the beginning of the proof, we can just apply the argument in [38].) In this setting there is a $\mathbb{Z} / 2 \mathbb{Z}$ grading

$$
R(d \gamma)^{\vee}=R(d \gamma)_{\text {even }}^{\vee} \cup R(d \gamma)_{\text {odd }}^{\vee}
$$

on the integral coroots $R(d \gamma)^{\vee}$ ([38], Lemma 8.6.3); the odd coroots in this grading (and also the corresponding roots) are said to satisfy the parity condition. Attached to every odd positive root $\beta$ there is a "Cayley transformed parameter"

$$
\Gamma_{\beta}=\left(H_{\beta}, \Gamma_{\beta},\{\tilde{\beta}\}\right) \quad\left(\beta \in R^{+}(d \gamma)_{\text {odd }}\right) .
$$

Here $H_{\beta} \subset \phi_{\beta}(S L(2, \mathbb{R})) \cdot H$ (Definition 5.2) corresponds to the Cartan subgroup $S O(2)$ of $S L(2, \mathbb{R})$; its only imaginary roots are the two $\pm \tilde{\beta}$ in $\phi_{\beta}(\mathfrak{s l}(2, \mathbb{C}))$. This is essentially the Langlands parameter of the discrete series representation of $S L(2, \mathbb{R})$ appearing inside a reducible principal series representation. (The "type II" assumption, which concerns the disconnectedness of $H$, means that this discrete series representation is unique, rather than being one of a pair; that is the "simplified notation" of which we are taking advantage.)

Because $H$ is assumed to be split, the Cartan involution $\theta$ of $\mathfrak{h}$ is -1 (at least on the span of the roots). The Cartan involution $\theta_{\beta}$ on $\mathfrak{h}_{\beta}$ is therefore $-s_{\tilde{\beta}}$ (again on the span of the roots). 
The long intertwining operator for $I(\Gamma)$ has a factorization in which each factor corresponds to a real root. The factors for the nonintegral roots, and for the integral roots of even grading, are all isomorphisms, and so do not contribute to the kernel of the long intertwining operator. The conclusion is that the composition factors of the kernel - that is, the composition factors of $I(\Gamma)$ other than $J(\Gamma)$-all appear in $I\left(\Gamma_{\beta}\right)$ for some (odd positive integral real) $\beta$.

In terms of the Bruhat order, this means

$$
\Xi<\Gamma \Longleftrightarrow \Xi \leq \Gamma_{\beta} \quad\left(\text { some } \beta \in R^{+}(d \gamma)_{\text {odd }}\right)
$$

It is easy to compute

$$
\ell\left(\Gamma_{\beta}\right)=\ell(\Gamma)-\left[\ell\left(s_{\beta}\right)+1\right] / 2,
$$

where the length function in brackets is the one for the integral Weyl group $W(R(d \gamma))$. The only elements of a Weyl group of length one are the simple reflections; so

$$
\ell\left(\Gamma_{\beta}\right) \leq \ell(\Gamma)-1,
$$

with equality if and only if $\beta$ is simple in $R^{+}(d \gamma)$.

The conclusion is that the only possible immediate predecessors of $\Gamma$ are the various $\Gamma_{\beta}$, with $\beta$ odd positive integral. To complete the proof of the proposition, we must show that if $\beta$ is not simple, then $\Gamma_{\beta}$ is not an immediate predecessor. To prove this, we will show

if $\beta$ is odd positive, there is a simple odd $\alpha$ with $\Gamma_{\beta} \leq \Gamma_{\alpha}$.

\{eq: bruhatheight $\}$

This we prove by induction on the height of $\beta$. If $\beta$ is simple, then we can take $\alpha=\beta$; so suppose $\beta$ is not simple. What we need to prove is

if $\beta$ is odd positive not simple, there is

an odd positive $\beta^{\prime}$ with $\Gamma_{\beta}<\Gamma_{\beta^{\prime}}$.

It is convenient to prove the stronger statement

if $\beta$ is odd positive not simple, there is an odd positive $\beta^{\prime}$ with $\Gamma_{\beta} \not \Gamma_{\beta^{\prime}}, J\left(\Gamma_{\beta}\right) \subset I\left(\Gamma_{\beta^{\prime}}\right)$.

Here $\subset$ is abused to mean "is a composition factor of." Choose a simple root $\delta$ so that

$$
\left\langle\beta, \delta^{\vee}\right\rangle=m>0
$$


Since $\beta$ is not simple, $\delta \neq \beta$; so

$$
s_{\delta}(\beta)=\beta-m \delta=\beta^{\prime \prime}
$$

is a positive root of lower height than $\beta$. By a similar calculation, the root $\tilde{\delta}$ for $H_{\beta}$ corresponding to $\delta$ is complex, and $\theta_{\beta}(\tilde{\delta})$ is negative. Consequently $\delta \notin \tau\left(\Gamma_{\beta}\right)$ (see Theorem 16.6). The Kazhdan-Lusztig algorithm (in fact the more elementary results proved in [39]) shows that $J\left(\Gamma_{\beta}\right)$ is a composition factor of $I\left(s_{\delta} \times \Gamma_{\beta}\right)$, so that

$$
J\left(\Gamma_{\beta}\right) \subset I\left(s_{\delta} \times \Gamma_{\beta}\right), \quad \ell\left(\Gamma_{\beta}\right)=\ell\left(s_{\delta} \times \Gamma_{\beta}\right)-1 .
$$

On the other hand, an elementary calculation shows that

$$
s_{\delta} \times \Gamma_{\beta} \simeq\left(s_{\delta} \times \Gamma\right)_{\beta^{\prime \prime}} .
$$

(The notation is slightly imprecise. As a representative for the equivalence class $s_{\delta} \times \Gamma$, we choose the one having the same differential as $\Gamma$; that is, we apply some shift by a multiple of $\delta$, then conjugate by the real Weyl group reflection in $\delta$.)

We now consider two cases. First, if $\delta$ is even, then $s_{\delta} \times \Gamma=\Gamma$, and so we have shown that

$$
J\left(\Gamma_{\beta}\right) \subset I\left(s_{\delta} \times \Gamma_{\beta}\right)=I\left(\Gamma_{\beta^{\prime \prime}}\right) .
$$

This is $(18.11 \mathrm{~h})$, with $\beta^{\prime}=\beta^{\prime \prime}$.

Next suppose $\delta$ is odd. In order to prove (18.11h), we can consider the (semisimple) rank two reductive subgroup $L$ of $G$ generated by the rational span of the roots $\delta$ and $\beta$ and the split Cartan $H$. This is a Levi subgroup of a real parabolic (not necessarily containing our fixed Borel). By induction by stages, one sees that it suffices to prove $(18.11 \mathrm{~h})$ for $G=L$. The setting is therefore that $G$ is split and simple of rank two, $\Gamma$ is attached to the split Cartan, the simple root $\delta$ is odd, and the non-simple root $\beta$ is also odd.

In types $A_{2}$ and $G_{2}$, and in the block for $B_{2}$ with no finite-dimensional representations, every non-discrete series block element of length at most $\ell(\Gamma)-2\left(\right.$ like $\left.J\left(\Gamma_{\beta}\right)\right)$ is a composition factor of every $I(\Xi)$ with $\ell(\Xi)=\ell(\Gamma)-1$ (like $\left.I\left(\Gamma_{\delta}\right)\right)$. So $(18.11 \mathrm{~h})$ is true with $\beta^{\prime}=\delta$.

We may therefore assume that $L$ is of type $B_{2}$, and that the block contains finite-dimensional representations. Since $\delta$ is assumed to be odd, there are just two possibilities.

a) The simple root $\delta$ is long, the other simple root $\delta^{\prime}$ is short, $\beta=\delta+\delta^{\prime}$ is short, and $\beta^{\vee}=2 \delta^{\vee}+\left(\delta^{\prime}\right)^{\vee}$. 
b) The simple root $\delta$ is short, $\beta=2 \delta+\delta^{\prime}$ is long, and $\beta^{\vee}=\delta^{\vee}+\left(\delta^{\prime}\right)^{\vee}$.

In case (a), since $\beta$ is assumed to be odd, $\delta^{\prime}$ must also be odd; so both simple roots are odd for $\Gamma$. In this case it turns out that in fact $s_{\delta} \times \Gamma_{\beta}$ may not precede $\Gamma$ in the Bruhat order; so $(18.11 \mathrm{k})$ is of no help to us. Instead one can compute that $J\left(\Gamma_{\beta}\right)$ is a composition factor of $I\left(\Gamma_{\delta}\right)$; so we get (18.11h) with $\beta^{\prime}=\delta$.

In case (b), since $\beta$ and $\delta$ are assumed to be odd, $\delta^{\prime}$ must be even. Again it turns out that $s_{\delta} \times \Gamma_{\beta}$ may not precede $\Gamma$ in the Bruhat order, but that $J\left(\Gamma_{\beta}\right)$ is a composition factor of $I\left(\Gamma_{\delta}\right)$.

From (18.11f) it is clear that the only candidates for immediate predecessors of $\Gamma$ are the $\Gamma_{\alpha}$, with $\alpha$ odd and simple. These have length one less, as the Proposition requires.

What we are looking for is a " $q$-analogue" of the multiplicity of $J(\Xi)$ in $I(\Gamma)$; that is, a collection of (meaningful!) integers that sum to the multiplicity. The Jantzen filtration of $I(\Gamma)$ is perfectly designed for this.

Definition 18.12. Suppose $\Gamma$ and $\Xi$ are Langlands parameters. Recall $\{$ def : multpoly $\}$ from $(14.5 \mathrm{~d})$ the finite decreasing filtration

$$
I_{\text {quo }}(\Gamma)=I_{\text {quo }}(\Gamma)^{0} \supset I_{\text {quo }}(\Gamma)^{1} \supset I_{\text {quo }}(\Gamma)^{2} \cdots
$$

We define the multiplicity polynomial so that the coefficients record the multiplicities in the subquotients of this filtration: by

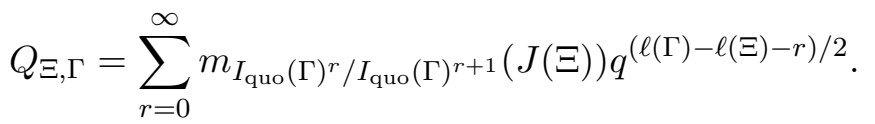

This is a finite Laurent polynomial in the formal variable $q^{1 / 2}$. The value at $q^{1 / 2}=1$ of this Laurent polynomial is the multiplicity of $J(\Xi)$ in $I(\Gamma)$ :

$$
Q_{\Xi, \Gamma}(1)=m_{I_{\mathrm{quo}}(\Gamma)}(J(\Xi))=m_{\Xi, \Gamma}
$$

(see (15.1f))

This definition is normalized to make $Q_{\Xi, \Gamma}$ a polynomial in $q$. It would be more natural to consider

$$
\left(q^{-1 / 2}\right)^{(\ell(\Gamma)-\ell(\Xi))} Q_{\Xi, \Gamma}=\sum_{r=0}^{\infty} m_{I_{\text {quo }}(\Gamma)^{r} / I_{\text {quo }}(\Gamma)^{r+1}}(J(\Xi)) q^{-r / 2} .
$$

This definition carries over to the extended group ${ }^{\delta} G$ without change. 
Although the multiplicity polynomial is the one we are most interested in, it seems aesthetically satisfactory to include the dual definition.

Definition 18.13. Suppose $\Gamma$ and $\Psi$ are Langlands parameters of regular infinitesimal character. Write $H=T A$ for the Cartan subgroup attached to $\Gamma$. Choose as in (9.3) a $\theta$-stable parabolic subalgebra

$$
\mathfrak{q}=\mathfrak{l}+\mathfrak{u}
$$

of $\mathfrak{g}$, with $L$ the centralizer in $G$ of $T_{0}$, of type $\mathrm{VZ}$ with respect to $\Gamma$ (see $(9.3 \mathrm{~b})$ ). Finally recall (still from (9.3)) the Langlands parameter $\Gamma_{\mathfrak{q}}$ for $L$. We define the character polynomial by

$$
P_{\Gamma, \Psi}=\sum_{r=0}^{\infty}\left[\text { mult. of } J\left(\Gamma_{\mathfrak{q}}\right) \text { in } H^{s+r}(\overline{\mathfrak{u}},(J(\Psi))]\left(-q^{1 / 2}\right)^{(\ell(\Psi)-\ell(\Gamma)-r)},\right.
$$

a finite Laurent polynomial in the formal variable $q^{1 / 2}$.

The preceding definition is normalized to make $P_{\Gamma, \Psi}$ a polynomial with nonnegative coefficients. It would be more natural to consider

$$
\left(-q^{-1 / 2}\right)^{\ell(\Psi)-\ell(\Gamma)} P_{\Gamma, \Psi}=\sum_{r=0}^{\infty}(-1)^{r}\left[\text { mult. } J\left(\Gamma_{\mathfrak{q}}\right) \text { in } H^{s+r}(\overline{\mathfrak{u}},(J(\Psi))] q^{-r / 2} .\right.
$$

This definition carries over unchanged to the extended group ${ }^{\delta} G$.

We turn now to the theorems about computing the polynomials $P$ and $Q$. These do not extend painlessly to ${ }^{\delta} G$.

Theorem 18.14 (Lusztig-Vogan [41]). Suppose $\Gamma$ and $\Psi$ are Langlands pa$\{\mathrm{thm}: \mathrm{KLpol}\}$ rameters of regular infinitesimal character. Then the character polynomial $P_{\Gamma, \Psi}$ of Definition 18.13 is equal to the Kazhdan-Lusztig character polynomial (see [41]). In particular, it is a polynomial in $q$. Therefore

1. $J\left(\Gamma_{\mathfrak{q}}\right)$ can occur as a composition factor in $H^{s+r}(\overline{\mathfrak{u}}, J(\Psi))$ only if

(a) $\Gamma \leq \Psi$ in the Bruhat order (Definition 18.5); and

(b) $\ell(\Psi)-\ell(\Gamma)$ is congruent to $r$ modulo 2 ; and

(c) $0 \leq[(\ell(\Psi)-\ell(\Gamma))-r] / 2 \leq[\ell(\Psi)-\ell(\Gamma)] / 2$.

2. Equality can hold in the second inequality of 1(c) only if $\Psi=\Gamma$. Equivalently,

$$
\operatorname{deg} P_{\Gamma, \Psi} \leq[\ell(\Psi)-\ell(\Gamma)-1] / 2, \quad \text { all } \Gamma<\Psi .
$$


Here is the corresponding result about the cohomology of standard modules. Write a subscript $\Gamma$ to denote projection on the summand of infinitesimal character equal to that of $J\left(\Gamma_{\mathfrak{q}}\right)$.

3.

$$
H^{s+r}\left(\overline{\mathfrak{u}}, I_{\mathrm{sub}}(\Psi)\right)_{\Gamma} \simeq \begin{cases}I_{\mathrm{sub}}\left(\Gamma_{\mathfrak{q}}\right) & \Gamma=\Psi, \quad r=0 \\ 0 & \text { otherwise }\end{cases}
$$

4. In the Grothendieck group of virtual $\left(\mathfrak{g}_{0}, K\right)$-modules (cf. (15.1)),

$$
[J(\Psi)]=\sum_{\Gamma}(-1)^{\ell(\Psi)-\ell(\Gamma)} P_{\Gamma, \Psi}(1)[I(\Gamma)] .
$$

Theorem 18.15 (Beilinson-Bernstein [3]). Suppose $\Gamma$ and $\Xi$ are Langlands parameters of regular infinitesimal character. Then the multiplicity polynomial $Q_{\Xi, \Gamma}$ of Definition 18.12 is equal to the Kazhdan-Lusztig "multiplicity polynomial" obtained by inverting the Kazhdan-Lusztig matrix $\left(P_{\Gamma, \Psi}\right)$ (see [42], Sections 1 and 12). In particular, it is a polynomial in $q$. Therefore

1. $J(\Xi)$ can occur as a composition factor in $I_{\text {quo }}(\Gamma)^{r} / I_{\text {quo }}(\Gamma)^{r+1}$ only if

(a) $\Xi \leq \Gamma$ in the Bruhat order (Definition 18.5); and

(b) $\ell(\Gamma)-\ell(\Xi)$ is congruent to $r$ modulo 2 ; and

(c) $0 \leq[(\ell(\Gamma)-\ell(\Xi))-r] / 2 \leq[\ell(\Gamma)-\ell(\Xi)] / 2$.

2. Equality can hold in the second inequality of 1(c) only if $\Gamma=\Xi$. Equivalently,

$$
\operatorname{deg} Q_{\Xi, \Gamma} \leq[\ell(\Gamma)-\ell(\Xi)-1] / 2, \quad \text { all } \Xi<\Gamma .
$$

3. In the Grothendieck group of virtual $\left(\mathfrak{g}_{0}, K\right)$-modules (cf. (15.1)),

$$
[I(\Gamma)]=\sum_{\Xi} Q_{\Xi, \Gamma}(1) J(\Xi)
$$

We conclude this section with the corresponding results for singular infinitesimal character.

Corollary 18.16. Suppose $\lambda_{0}$ is a possibly singular infinitesimal character, and $\phi$ is a weight of a finite-dimensional representation of $G$ chosen as in (16.8), so that in particular the infinitesimal character $\lambda_{1}=\lambda_{0}+\phi$ is regular. Write

$$
t_{\lambda_{0}}^{\lambda_{0}+\phi}: B\left(\lambda_{0}\right) \hookrightarrow B\left(\lambda_{0}+d \phi\right)
$$


as in Corollary 16.9 for the corresponding inclusion of Langlands parameters. Fix Langlands parameters

$$
\Gamma_{0}, \Xi_{0} \in B\left(\lambda_{0}\right)
$$

and write

$$
\Gamma=t_{\lambda_{0}}^{\lambda_{0}+\phi}\left(\Gamma_{0}\right), \quad \Xi=t_{\lambda_{0}}^{\lambda_{0}+\phi}\left(\Xi_{0}\right)
$$

for the corresponding regular parameters in $B\left(\lambda_{0}+\phi\right)$. Then the multiplicity polynomials of Definition 18.12 satisfy

$$
Q_{\Xi_{0}, \Gamma_{0}}=Q_{\Xi, \Gamma}
$$

That is, the multiplicity polynomials at singular infinitesimal character are a subset of the (Kazhdan-Lusztig) multiplicity polynomials at regular infinitesimal character; the subset is where both indices have no root of $\Pi^{\lambda_{0}}$ (notation (16.8); that is, no root singular on $\lambda_{0}$ ) in the $\tau$-invariant (Definition 16.7).

\section{KLV theory for extended groups}

In this section we fix again an extended group

$$
{ }^{\delta} G=G \rtimes\left\{1, \delta_{f}\right\}
$$

as in Definition 12.3. Fix a maximally split extended torus ${ }^{1} H_{s}$ as in (17.8), and a weight $\lambda_{0} \in \mathfrak{h}_{s}^{*}$ satisfying the dominance condition (17.8g). We want information about representations of ${ }^{\delta} G$-precisely, about irreducible $\left(\mathfrak{g},{ }^{\delta} K\right)$-modules-annihilated by $\left[\operatorname{ker} \xi_{\lambda_{0}}\right]\left[\operatorname{ker} \xi_{t_{s} \lambda_{0}}\right]$; these are parametrized by the extended Langlands parameters from (17.8i)

$$
{ }^{\delta} B\left(\lambda_{0}\right)={ }^{\delta} B\left(\lambda_{0}\right)^{1} \amalg{ }^{\delta} B\left(\lambda_{0}\right)^{2},
$$

where the (coproduct) notation indicates the disjoint union into type one and type two parameters (Definition 13.7).

A word about notation: we prefer to write a type two parameter for ${ }^{\delta} G$ as $\Gamma_{\text {ind }}$ (with $\Gamma$ a type two parameter for $G$, and a type one parameter as $\Gamma_{+}$ or $\Gamma_{-}$. When we are discussing "generic" parameters for ${ }^{\delta} G$ (not specified to be type one or type two) we will use Roman letters $x, y, z \ldots$.

We will use the corresponding sets of Langlands parameters for $G$ corresponding to ${ }^{\delta} B\left(\lambda_{0}\right)$, namely

$$
B\left(\lambda_{0}\right)=B\left(\lambda_{0}\right)^{1} \amalg B\left(\lambda_{0}\right)^{2} ;
$$


recall that a type one representation of $G$ is one fixed by twisting by $\theta$, and a type one Langlands parameter is one equivalent (that is, conjugate by a real Weyl group element) to its twist by $\theta$.

Definition 18.12 for multiplicity polynomials makes it clear how tensoring with the character $\xi$ (Definition 8.12) (or by any other one-dimensional character of $\left.\left(\mathfrak{g},{ }^{\delta} K\right)\right)$ affects them:

$$
Q_{\xi \otimes x, \xi \otimes y}=Q_{x, y} \quad\left(x, y \in{ }^{\delta} B\left(\lambda_{0}\right)\right) .
$$

$\{$ eq:Qxi\}

For the character polynomials of Definition 18.13 the result is

$$
P_{\xi \otimes y, \xi \otimes z}=P_{y, z} \quad\left(y, z \in{ }^{\delta} B\left(\lambda_{0}\right), \lambda_{0} \text { regular }\right) .
$$

There are corresponding assertions about twisting by $\theta$ (or by any other automorphism of $(\mathfrak{g}, K))$ on $G$ :

$$
\begin{gathered}
Q_{\Xi^{\theta}, \Gamma^{\theta}}=Q_{\Xi, \Gamma} \quad\left(\Xi, \Gamma \in B\left(\lambda_{0}\right)\right), \\
P_{\Gamma^{\theta}, \Psi^{\theta}}=P_{\Gamma, \Psi} \quad\left(\Gamma, \Psi \in B\left(\lambda_{0}\right), \lambda_{0} \text { regular }\right) .
\end{gathered}
$$

\{eq:Qtheta\}

$\{$ eq: Ptheta $\}$

We first say a few words about the (essentially trivial) case

$$
t_{s}\left(\lambda_{0}\right) \neq \lambda_{0},
$$

or equivalently $\xi_{\lambda_{0}} \neq \xi_{\lambda_{0}}^{\theta}$. In this case twisting by $\theta$ defines a bijection

$$
\theta: B\left(\lambda_{0}\right) \stackrel{\sim}{\longrightarrow} B\left(t_{s} \lambda_{0}\right)
$$

between the disjoint sets $B\left(\lambda_{0}\right)$ and $B\left(t_{s} \lambda_{0}\right)$. Therefore induction of parameters is a bijection

$$
\text { ind: } B\left(\lambda_{0}\right) \stackrel{\sim}{\longrightarrow}{ }^{\delta} B\left(\lambda_{0}\right)^{2}={ }^{\delta} B\left(\lambda_{0}\right), \quad \Gamma \mapsto \Gamma_{\text {ind }} .
$$

In this case (by Clifford theory) all standard representations and all irreducible representations are induced from $G$ to ${ }^{\delta} G$. It is very easy to check from the definition of the Jantzen filtration that the filtration is also induced:

$$
I_{\text {quo }}\left(\Gamma_{\text {ind }}\right)^{r}=\operatorname{Ind}_{(\mathfrak{g}, K)}^{(\mathfrak{g}, \delta)}\left(I_{\text {quo }}(\Gamma)^{r}\right) .
$$

From this it follows immediately that

$$
Q_{\Xi_{\text {ind }}, \Gamma_{\text {ind }}}=Q_{\Xi, \Gamma}+Q_{\Xi^{\theta}, \Gamma} \quad\left(\Xi, \Gamma \in B\left(\lambda_{0}\right)\right)
$$


Since $\Xi^{\theta}$ has the wrong infinitesimal character to appear in $I(\Gamma)$, the second term on the right is zero, and we get

$$
\left.Q_{\Xi_{\text {ind }}, \Gamma_{\text {ind }}}=Q_{\Xi, \Gamma} \quad\left(\Xi, \Gamma \in B\left(\lambda_{0}\right), \quad \lambda_{0} \neq t_{s} \lambda_{0}\right) . \quad \text { (19.1m) } \quad \text { eq:Qall2 }\right\}
$$

By a slightly more technical but equally easy computation with Lie algebra cohomology, we find

$$
P_{\Gamma_{\text {ind }}, \Psi_{\text {ind }}}=P_{\Gamma, \Psi} \quad\left(\Gamma, \Psi \in B\left(\lambda_{0}\right), \quad \lambda_{0} \neq t_{s} \lambda_{0} \text { regular }\right) .
$$

Having disposed of this easy case, we will assume for the rest of this section that

$$
t_{s}\left(\lambda_{0}\right)=\lambda_{0} .
$$

What Clifford theory says is that

$$
\text { ind: } B\left(\lambda_{0}\right)^{2} \rightarrow{ }^{\delta} B\left(\lambda_{0}\right)^{2} \text { is a two-to-one surjection, }
$$

and

$$
\text { res: }{ }^{\delta} B\left(\lambda_{0}\right)^{1} \rightarrow B\left(\lambda_{0}\right)^{1} \text { is a two-to-one surjection. }
$$

The fibers of the first surjection are the orbits of the twisting action by $\theta$, and the fibers of the second surjection are the orbits of tensoring with the nontrivial character $\xi$ of ${ }^{\delta} G / G$ (Definition 8.12).

We will also make use of a type one weight as in (17.8p):

$$
\phi \in \Lambda_{\mathrm{fin}}\left(G, H_{s}\right)^{t_{s}}
$$

of a finite-dimensional representation of $G$, dominant for $R_{s}^{+}$, and such that

$$
\lambda_{0}+d \phi \text { is dominant and regular for } R^{+}\left(\lambda_{0}\right) .
$$

Parallel to Corollary 16.9(4), we get from Corollary 17.9(4) an inclusion

$$
t_{\lambda_{0}}^{\lambda_{0}+\phi_{+}}:{ }^{\delta} B\left(\lambda_{0}\right) \hookrightarrow{ }^{\delta} B\left(\lambda_{0}+d \phi\right),
$$

allowing us (parallel to Theorem 18.15) to compute the Kazhdan-Lusztig multiplicity polynomials $Q$ at the (possibly singular) infinitesimal character $\lambda_{0}$ as a subset of those at the (regular) infinitesimal character $\lambda_{0}+d \phi$.

With notation established, we turn to computation of the KazhdanLusztig character polynomials $P_{x, y}$ for ${ }^{\delta} G$ at regular infinitesimal character. Recall (Definition 18.13) that such a polynomial describes certain constituents of the Lie algebra cohomology of the irreducible representation $J(y)$; the constituents are related to the standard representation $I(x)$. We 
consider four cases separately, according to the types of $x$ and $y$. First, assume that $y=\Psi_{\text {ind }}$ is type two, so that the irreducible representation $J(y)$ is induced from $J(\Psi)$ on $G$. The Lie algebra cohomology of this induced representation is easy to compute, and we get just as in $(19.1 \mathrm{~m})$ above (always assuming $\lambda_{0}$ regular)

$$
\begin{aligned}
& P_{\Gamma_{\text {ind }}, \Psi_{\text {ind }}}=P_{\Gamma, \Psi}+P_{\Gamma, \Psi^{\theta}} \\
& =P_{\Gamma^{\theta}, \Psi}+P_{\Gamma^{\theta}, \Psi^{\theta}} \quad\left(\Gamma \in B\left(\lambda_{0}\right)^{2}, \Psi \in B\left(\lambda_{0}\right)^{2}\right) \\
& P_{\Gamma_{ \pm}, \Psi_{\text {ind }}}=P_{\Gamma, \Psi} \\
& =P_{\Gamma, \Psi^{\theta}} \quad\left(\Gamma \in B\left(\lambda_{0}\right)^{1}, \Psi \in B\left(\lambda_{0}\right)^{2}\right)
\end{aligned}
$$

$\{$ eq:PGamma2Psi2\}

\{eq:PGamma1Psi2\}

the last equality is a consequence of $(19.1 \mathrm{~g})$, and the hypothesis $\Gamma^{\theta} \simeq \Gamma$. Next, assume that $y=\Psi_{ \pm}$is type one, so that $J\left(\Psi_{ \pm}\right)$is an extension to ${ }^{\delta} G$ of the irreducible representation $J(\Psi)$. If $x=\Gamma_{\text {ind }}$ is type two, then $P_{\Psi_{ \pm}, \Gamma_{\text {ind }}}$ counts multiplicities of a certain induced representation in the Lie algebra cohomology of $J\left(\Psi_{ \pm}\right)$. By Frobenius reciprocity, such a multiplicity can be computed after restriction to $G$ (still assuming $\lambda_{0}$ regular):

$$
\begin{aligned}
P_{\Gamma_{\text {ind }}, \Psi_{ \pm}} & =P_{\Gamma, \Psi} \\
& =P_{\Gamma^{\theta}, \Psi} \quad\left(\Gamma \in B\left(\lambda_{0}\right)^{2}, \Psi \in B\left(\lambda_{0}\right)^{1}\right) .
\end{aligned}
$$

\{eq:PPsi1Gamma2\}

We record also the more elementary analogues for the multiplicity polynomials $Q$, which do not require the hypothesis of regular infinitesimal character:

$$
\begin{aligned}
Q_{\Xi_{\text {ind }}, \Gamma_{\text {ind }}} & =Q_{\Xi, \Gamma}+Q_{\Xi, \Gamma^{\theta}} & & \\
& =Q_{\Xi^{\theta}, \Gamma}+Q_{\Xi^{\theta}, \Gamma^{\theta}} & & \left(\Xi \in B\left(\lambda_{0}\right)^{2}, \Gamma \in B\left(\lambda_{0}\right)^{2}\right) ; \\
Q_{\Xi_{ \pm}, \Gamma_{\text {ind }}} & =Q_{\Xi, \Gamma}=Q_{\Xi, \Gamma^{\theta}} & & \left(\Xi \in B\left(\lambda_{0}\right)^{1}, \Gamma \in B\left(\lambda_{0}\right)^{2}\right) ; \\
Q_{\Xi_{\text {ind }}, \Gamma_{ \pm}} & =Q_{\Xi, \Gamma}=Q_{\Xi^{\theta}, \Gamma} & & \left(\Xi \in B\left(\lambda_{0}\right)^{2}, \Gamma \in B\left(\lambda_{0}\right)^{1}\right) .
\end{aligned}
$$

\{eq:QXi2Gamma2\}

\{eq:QXi1Gamma2\}

\{eq:QXi2Gamma1\}

In all of these cases - that is, whenever one of the indices is type two - the Kazhdan-Lusztig polynomials for ${ }^{\delta} G$ are given by extremely simple formulas in terms of the Kazhdan-Lusztig polynomials for $G$. The remaining case is when both indices are type one. We will therefore be considering parameters

$$
\Xi, \Gamma, \Psi \in B\left(\lambda_{0}\right)^{1}
$$

and their extensions

$$
\Xi_{ \pm}, \Gamma_{ \pm}, \Psi_{ \pm} \in{ }^{\delta} B\left(\lambda_{0}\right)^{1}
$$


These pairs (and the corresponding representations) are interchanged by tensoring with $\xi$ (Definition 8.12):

$$
\xi \otimes J\left(\Xi_{+}\right)=J\left(\Xi_{-}\right), \quad \xi \otimes J\left(\Xi_{-}\right)=J\left(\Xi_{+}\right) .
$$

Using (19.1e), we deduce

$$
P_{\Gamma_{ \pm}, \Psi_{-}}=P_{\Gamma_{\mp}, \Psi_{+}} \quad\left(\Gamma, \Psi \in B\left(\lambda_{0}\right)^{1}, \lambda_{0} \text { regular }\right),
$$

and similarly

$$
Q_{\Xi_{ \pm}, \Gamma_{-}}=Q_{\Xi_{\mp}, \Gamma_{+}} \quad\left(\Xi, \Gamma \in B\left(\lambda_{0}\right)^{1}\right) .
$$

So it is enough to compute all of the polynomials $P$ and $Q$ with the second index labeled + . (The same statement would be true with all four variations on "first" and "second," but this is the one we prefer.)

The polynomials $P_{\Gamma_{ \pm}, \Psi_{+}}$describe the occurrence of certain representations of an extended group ${ }^{\delta} L$ (depending on $\Gamma$ ) in the Lie algebra cohomology of $J\left(\Psi_{+}\right)$. By restriction to $G$, we see that

$$
P_{\Gamma_{+}, \Psi_{+}}+P_{\Gamma_{-}, \Psi_{+}}=P_{\Gamma, \Psi} \quad\left(\Gamma, \Psi \in B\left(\lambda_{0}\right)^{1}, \lambda_{0} \text { regular }\right),
$$

That is, the sum of these two character polynomials for ${ }^{\delta} G$ that we wish to compute is a (known) character polynomial for $G$. It is therefore natural to consider the difference

$$
P_{\Gamma, \Psi}^{\delta}={ }_{\text {def }} P_{\Gamma_{+}, \Psi_{+}}-P_{\Gamma_{-}, \Psi_{+}} \quad\left(\Gamma, \Psi \in B\left(\lambda_{0}\right)^{1}, \lambda_{0} \text { regular }\right) .
$$

$\{$ eq: twcharpoly $\}$

The polynomial $P_{\Gamma, \Psi}^{\delta}$ is called a twisted character polynomial. What the preceding formulas say is that if we can compute all the twisted character polynomials $P_{\Gamma, \Psi}^{\delta}$, we will know all the character polynomials for ${ }^{\delta} G$.

In exactly the same way, the polynomials $Q_{\Xi_{ \pm}, \Gamma_{+}}$describe the occurrence of the the irreducible representations $J\left(\Xi_{ \pm}\right)$in the Jantzen filtration of of $I\left(\Gamma_{+}\right)$. By restriction to $G$, we see that

$$
Q_{\Xi_{+}, \Gamma+}+Q_{\Xi_{-} \Gamma_{+}}=Q_{\Xi, \Gamma} \quad\left(\Xi, \Gamma \in B\left(\lambda_{0}\right)^{1}\right),
$$

That is, the sum of these two multiplicity polynomials for ${ }^{\delta} G$ that we wish to compute is a (known) multiplicity polynomial for $G$. It is therefore natural to consider the difference

$$
Q_{\Xi, \Gamma}^{\delta}={ }_{\operatorname{def}} Q_{\Xi_{+} \Gamma_{+}}-Q_{\Xi_{-} \Gamma_{+}} \quad\left(\Gamma, \Psi \in B\left(\lambda_{0}\right)^{1}\right),
$$

$\{$ eq: twmultpoly $\}$

a twisted multiplicity polynomial. Again what is true is if we can compute all the twisted multiplicity polynomials $Q_{\Gamma, \Psi}^{\delta}$, we will know all the multiplicity polynomials for ${ }^{\delta} G$.

Here is the main theorem of this section. 
$\{$ thm:twKLpol $\}$

Theorem 19.4 (Lusztig-Vogan [27]). Suppose $\Gamma, \Psi \in B\left(\lambda_{0}\right)^{1}$ are type one Langlands parameters of regular $\theta$-stable infinitesimal character $\lambda_{0}$. Then the twisted character polynomial $P_{\Gamma, \Psi}^{\delta}$ of $(19.3 \mathrm{~g})$ is equal to the polynomial defined in [27]. In particular, it is a polynomial in $q$ with integer coefficients. Each coefficient is bounded in absolute value by the corresponding coefficient of $P_{\Gamma, \Psi}$, and congruent to that coefficient modulo two.

What is actually written in [27] is a statement about equivariant perverse sheaves on a flag variety in finite characteristic. By standard base change results, this is equivalent to a statement about ${ }^{\delta} K(\mathbb{C})$-equivariant perverse sheaves on the flag variety of Borel subalgebras of $\mathfrak{g}$. By the Beilinson-Bernstein localization theory, with appropriate minor modifications as in [41], this becomes a statement about twisted character polynomials for $\left(\mathfrak{g},{ }^{\delta} K(\mathbb{C})\right)$-modules, which is precisely the theorem above in case $\lambda_{0}=\rho$, the half sum of a full set of positive roots.

The case of non-integral infinitesimal character can be treated just as for connected groups, using the ideas of Beilinson, Bernstein, Brylinski, Kashiwara, and Lusztig as sketched in Chapter 17 of [1]. (As far as we know, the ideas have been written down by their originators only in the first chapter of [25], which explains the case of Verma modules.)

\section{KLV theory for $c$-invariant forms}

In this section we fix a weight $\lambda_{0} \in \mathfrak{h}_{s}^{*}$ representing a real infinitesimal character (that is, belonging to the real span of the algebraic weight lattice $\left.X^{*}\right)$ as in Definition 16.7. In (15.12), we wrote finite matrices over $\mathbb{W}$ (Definition 15.9)

$$
\left(W_{\Gamma, \Psi}^{c}\right), \quad\left(w_{\Xi, \Gamma}\right) \quad\left(\Gamma, \Psi \in B\left(\lambda_{0}\right)\right)
$$

describing the signatures of $c$-invariant forms on irreducible representations in terms of those on standard representations. The ring $\mathbb{W}=\mathbb{Z}+s \mathbb{Z}$ comes equipped with a homomorphism (Definition 15.9

$$
\text { for }: \mathbb{W} \rightarrow \mathbb{Z}, \quad p+q s \mapsto p+q,
$$

and these signature matrices are related to the multiplicity matrices by

$$
\text { for }\left(w_{\Xi, \Gamma}\right)=m_{\Xi, \Gamma}, \quad \text { for }\left(W_{\Gamma, \Psi}^{c}\right)=M_{\Gamma, \Psi}
$$


In sections 18 and 19 we recalled the ideas of Kazhdan and Lusztig that led to computations of the multiplicity matrices $m$ and $M$ : introducing appropriate $q$-analogues $Q$ and $P$, and then computing those $q$-analogues using recursion relations that do not make sense at $q=1$. In the present section we will extend this to a computation of the signature matrices. We will need all this for extended groups. We will write only about $G$ to keep the notation under control, making comments as we go about the case of ${ }^{\delta} G$.

The first issue is to find $q$-analogues of $w$ and $W$. The first is easy.

Definition 20.2. In the setting of (20.1), suppose $\Gamma$ and $\Xi$ are Lang$\{$ def : wpoly $\}$ lands parameters in $B\left(\lambda_{0}\right)$. Recall from $(14.5 \mathrm{~d})$ the finite decreasing filtration

$$
I_{\text {quo }}(\Gamma)=I_{\text {quo }}(\Gamma)^{0} \supset I_{\text {quo }}(\Gamma)^{1} \supset I_{\text {quo }}(\Gamma)^{2} \cdots,
$$

and the nondegenerate Jantzen forms $\langle,\rangle^{[r]}$ on the subquotients of the filtration. Using Proposition 15.11, write in the Hermitian Grothendieck group

$$
\left[I_{\text {quo }}(\Gamma)^{r} / I_{\text {quo }}(\Gamma)^{r+1},\langle,\rangle^{[r]}\right]=\sum_{\Xi \in B\left(\lambda_{0}\right)} w_{\Xi, \Gamma}^{c, r}\left[J(\Xi),\langle,\rangle_{J(\Xi), b}^{c}\right]
$$

with $w_{\Xi, \Gamma}^{c, r} \in \mathbb{W}$. We define the signature multiplicity polynomial

$$
Q_{\Xi, \Gamma}^{c}=q^{(\ell(\Gamma)-\ell(\Xi) / 2} \sum_{r=0}^{\infty} w_{\Xi, \Gamma}^{c, r} q^{-r / 2} \in \mathbb{W}\left[q^{1 / 2}, q^{-1 / 2}\right] .
$$

The value at $q^{1 / 2}=1$ of this Laurent polynomial is the signature multiplicity of $J(\Xi)$ in $I(\Gamma)$ defined in (15.12):

$$
Q_{\Xi, \Gamma}^{c}(1)=w_{\Xi, \Gamma}^{c} \cdot
$$

Applying the natural forgetful homomorphism

$$
\text { for }: \mathbb{W}\left[q^{1 / 2}, q^{-1 / 2}\right] \rightarrow \mathbb{Z}\left[q^{1 / 2}, q^{-1 / 2}\right]
$$

clearly (by Definition 18.12) gives

$$
\operatorname{for}\left(Q_{\Xi, \Gamma}^{c}\right)=Q_{\Xi, \Gamma}
$$

This definition applies to the extended group ${ }^{\delta} G$ without change. 
Proposition 20.3. Suppose we are in the setting of Definition 20.2.

1. The signature multiplicity polynomial $Q_{\Xi, \Gamma}^{c}$ is a polynomial in $\mathbb{W}[q]$ of degree less than or equal to $\ell(\Gamma)-\ell(\Xi)] / 2$. Its coefficients are nonnegative, in the sense that if $p+q s$ is a coefficient, then $p$ and $q$ belong to $\mathbb{N}$.

2. The diagonal entries of the matrix $Q^{c}$ are equal to one: $Q_{\Gamma, \Gamma}^{c}=1$.

3. The polynomial $Q_{\Xi, \Gamma}^{c}$ can be nonzero only if $\Xi \leq \Gamma$ in the Bruhat order of Definition 18.6.

4. Equality can hold in (1) only if $\Gamma=\Xi$. Equivalently,

$$
\operatorname{deg} Q_{\Xi, \Gamma}^{c} \leq[\ell(\Gamma)-\ell(\Xi)-1] / 2, \quad \text { all } \Xi<\Gamma .
$$

5. The matrix $Q^{c}$ is upper triangular with diagonal entries 1 , and consequently is invertible.

Parallel results hold for ${ }^{\delta} G$.

Proof. The nonnegativity of the coefficients is clear from the definition. Because of the nonnegativity and the last formula of Definition 20.2, $Q_{\Xi, \Gamma}^{c}$ and $Q_{\Xi, \Gamma}$ have exactly the same set of nonvanishing coefficients. Because $Q_{\Xi, \Gamma}$ is known (by the Beilinson-Bernstein theorem 18.15) to be a polynomial with a degree bound, the same conclusion follows for $Q_{\Xi, \Gamma}^{c}$.

Definition 20.4. In the setting of (20.1) and Definition 20.2, consider $\{$ def :Wpoly $\}$ the matrix $Q^{c}$ (with entries indexed by $B\left(\lambda_{0}\right)$ ) of polynomials in $\mathbb{W}[q]$. The signature character polynomial is

$$
P_{\Gamma, \Psi}^{c}=(-1)^{\ell(\Psi)-\ell(\Gamma)}\left[(\Gamma, \Psi) \text { entry of }\left(Q^{c}\right)^{-1}\right] .
$$

By Proposition 20.3, $P_{\Gamma, \Psi}^{c}$ is a polynomial in $q$ (with coefficients in $\mathbb{W}$ ) of degree at most $[\ell(\Psi)-\ell(\Gamma)] / 2$, with equality only if $\Psi=\Gamma$. The value at $q=1$ of this polynomial is the signature coefficient of $I(\Gamma)$ in the irreducible $J(\Psi)$ defined in $(15.12 \mathrm{n})$ :

$$
P_{\Gamma, \Psi}^{c}(1)=(-1)^{\ell(\Psi)-\ell(\Gamma)} W_{\Gamma, \Psi}^{c} .
$$

Applying the natural forgetful homomorphism

$$
\text { for }: \mathbb{W}[q] \rightarrow \mathbb{Z}[q]
$$


gives

$$
\text { for }\left(P_{\Gamma, \Psi}^{c}\right)=P_{\Gamma, \Psi}
$$

This definition applies to the extended group ${ }^{\delta} G$ without change.

It would certainly be good to find a direct definition of $P_{\Gamma, \Psi}^{c}$ parallel to the definition of $P_{\Gamma, \Psi}$ in Definition 18.13, perhaps in terms of some natural invariant form on Lie algebra cohomology. But we do not know how to do that.

With $q$-analogues of the signature matrices in hand, we can ask how the ideas of Kazhdan and Lusztig help in computing them. Kazhdan-Lusztig theory involves in a fundamental way the notion of length recalled in Definition 18.2, which involves only the integral roots. The behavior of invariant forms is also affected by roots which "were" integral at some deformed value of a continuous parameter. The following definition is a version of length which looks at those "previously integral" nonintegral roots.

Definition 20.5. Suppose $\Gamma=(\Lambda, \nu)$ is a Langlands parameter (6.5). Write $\gamma$ for the differential of $\Gamma$, a weight defining the infinitesimal character. The orientation number $\ell_{o}(\Gamma)$ is equal to the sum of

a) the number of pairs $(\alpha,-\theta \alpha)$ of complex nonintegral roots such that

$$
\left\langle\gamma, \alpha^{\vee}\right\rangle>0, \quad\left\langle\gamma,-\theta \alpha^{\vee}\right\rangle>0
$$

and

b) the number of real nonintegral roots $\beta$ such that $\left\langle\gamma, \beta^{\vee}\right\rangle>0$, and the integral part $\left[\left\langle\gamma, \beta^{\vee}\right\rangle\right]$ is even if $\Lambda_{\mathfrak{q}}\left(m_{\alpha}\right)=+1$, or odd if $\Lambda_{\mathfrak{q}}\left(m_{\alpha}\right)=-1$ (notation $(9.3 \mathrm{~d}))$.

Theorem 20.6. Suppose $\Gamma$ and $\Xi$ are Langlands parameters of (real) in$\{$ thm:KLsigpolQ $\}$ finitesimal character $\chi$. Then the signature multiplicity polynomial (Definition 20.2) is

$$
Q_{\Xi, \Gamma}^{c}=s^{\left(\ell_{o}(\Xi)-\ell_{o}(\Gamma)\right) / 2} Q_{\Xi, \Gamma}(s q) .
$$

In particular

1. if $\Xi$ and $\Gamma$ belong to a common block, then $\ell_{o}(\Xi) \equiv \ell_{o}(\Gamma)(\bmod 2)$;

2. if $J(\Xi)$ appears in level $r$ of the Jantzen filtration of $I_{\text {quo }}(\Gamma)$, then

$$
\text { (a) } r \equiv \ell(\Gamma)-\ell(\Xi)(\bmod 2) ; \text { and }
$$


(b) the rth Jantzen form restricted to $J(\Xi)$ is

$$
(-1)^{\left[(\ell(\Gamma)-\ell(\Xi))+\left(\ell_{o}(\Gamma)-\ell_{o}(\Xi)\right)\right] / 2}\langle,\rangle_{J(\Xi), b} ;
$$

3. if $\ell_{o}(\Gamma)-\ell_{o}(\Xi) \equiv 0(\bmod 4)$, then the signature multiplicity coefficient of $(15.12 \mathrm{~g})$ is

$$
w_{\Xi, \Gamma}^{c}=\left(\text { sum of even coeffs of } Q_{\Xi, \Gamma}\right)+s\left(\text { sum of odd coeffs of } Q_{\Xi, \Gamma}\right) ;
$$

4. if $\ell_{o}(\Gamma)-\ell_{o}(\Xi) \equiv 2(\bmod 4)$, then the signature multiplicity coefficient is

$$
w_{\Xi, \Gamma}^{c}=\left(\text { sum of odd coeffs of } Q_{\Xi, \Gamma}\right)+s\left(\text { sum of even coeffs of } Q_{\Xi, \Gamma}\right) .
$$

Exactly the same results hold for the extended group ${ }^{\delta} G$.

Sketch of proof. Of course the proof is modeled on the proof of the KazhdanLusztig conjectures in [41] and [26]. Part (1) is a fairly easy combinatorial statement. Part 2(a)) is a restatement of Theorem $18.151(\mathrm{~b})$, recorded here only as a reminder that the powers of -1 appearing below are integer powers. Parts 3 and 4 are just restatements of 2(b); so what requires proof is $2(\mathrm{~b})$.

The first step is to reduce to the case of regular infinitesimal character. For this, fix a weight $\lambda_{0} \in \mathfrak{h}_{s}^{*}$ representing $\xi$ as in (17.8). Choose $\phi_{ \pm}$as in (17.8).

Lemma 20.7. In the setting of (17.8), the finite-dimensional representation $F_{\phi_{+}}$admits a positive-definite c-invariant Hermitian forms that is unique up to positive scalar multiple. Consequently the translation functors

$$
T_{\lambda_{0}}^{\lambda_{0}+\phi_{+}}, \quad T_{\lambda_{0}+\phi_{+}}^{\lambda_{0}}
$$

of (17.8v) carry (nondegenerate) c-invariant Hermitian forms to (nondegenerate) c-invariant Hermitian forms.

Suppose in addition that the real dominance hypotheses of (17.8g) and (17.8r) are satisfied. Suppose $\Gamma_{0} \in{ }^{\delta} B\left(\lambda_{0}\right)$ is any (singular) Langlands parameter, and

$$
\Gamma_{1}=t_{\lambda_{0}}^{\lambda_{0}+d \phi}\left(\Gamma_{0}\right)^{\delta} B\left(\lambda_{0}+d \phi\right)
$$

is the corresponding (regular) parameter (Theorem 17.9(4)). Then

1. $T_{\lambda_{0}+\phi_{+}}^{\lambda_{0}}$ carries the canonical c-invariant Hermitian form on $I_{\mathrm{quo}}\left(\Gamma_{1}\right)$ to a positive multiple of the canonical c-invariant Hermitian form on $I_{\text {quo }}\left(\Gamma_{0}\right)$; 
2. $T_{\lambda_{0}+\phi_{+}}^{\lambda_{0}}$ carries the canonical (nondegenerate) c-invariant Hermitian form on $J\left(\Gamma_{1}\right)$ to a positive multiple of the canonical c-invariant Hermitian form on $J\left(\Gamma_{0}\right)$;

3. $T_{\lambda_{0}+\phi_{+}}^{\lambda_{0}}$ carries the Jantzen filtration on $I_{\mathrm{quo}}\left(\Gamma_{1}\right)$ to the Jantzen filtration on $I_{\text {quo }}\left(\Gamma_{0}\right)$; and

4. $T_{\lambda_{0}+\phi_{+}}^{\lambda_{0}}$ carries the Jantzen form on each level of $I_{\mathrm{quo}}\left(\Gamma_{1}\right)$ (Definition 14.9 to a positive multiple of the Jantzen form on the corresponding level of $I_{\text {quo }}\left(\Gamma_{0}\right)$.

Sketch of proof. The first assertion of the lemma is just Weyl's "unitary trick:" the $c$-invariant form on $F_{\phi_{+}}$is by definition preserved by a compact form of $G$, and therefore must be definite. The computation of the sign in (1) trivially reduces to the case of $G$. There (in light of Proposition 10.7 ) it depends on looking carefully at how lowest $K$-types are affected by translation functors. (This is where the "real dominance" hypotheses are needed.) Part (2) follows immediately.

For (3), recall how the Jantzen filtration is defined in Proposition 14.7. When the regular parameter $\Gamma_{1}=\left(\Lambda_{1}, \nu_{1}\right)$ is modified by a small dilation of the continuous parameter, all the real dominance hypotheses are preserved. Write $\nu_{1, s} \in \mathfrak{h}_{s}^{*}$ for the weight corresponding to $\nu_{1}$ identification $i\left(R_{s}^{+}, R^{+}\right)$ of Proposition 16.2. The one-parameter family of translation functors

$$
T_{\left(\lambda_{0}+\epsilon \nu_{1, s}\right)+\phi_{+}}^{\left(\lambda_{0}+\epsilon \nu_{1, s}\right)}
$$

(all of which use the same finite-dimensional representation; only the infinitesimal character projection changes) all carry irreducibles to irreducibles or zero for small $\epsilon$. These functors carry $I_{\text {quo }}\left(\Lambda_{1},(1+\epsilon) \nu_{1}\right)$ - the oneparameter family defining the Jantzen filtration of $I_{\text {quo }}\left(\Gamma_{1}\right)$ - to $I_{\text {quo }}\left(\Lambda_{0}, \nu_{0}+\right.$ $\left.\epsilon \nu_{1}\right)$. Although this second family is not precisely the one used to define the Jantzen filtration for $I_{\text {quo }}\left(\Gamma_{0}\right)$, it is close enough that one can show that the filtration is the same. This proves (3).

Parts (1) and (3) immediately imply (4).

The lemma allows us to deduce all of the assertions of the theorem $\{$ se:proofKLsigpolQ\} at possibly singular infinitesimal character $\lambda_{0}$ by applying the translation functor $T_{\lambda_{0}+\phi_{+}}^{\lambda_{0}}$ to the assertions at the regular infinitesimal character $\lambda_{0}+d \phi$. Just as in the proof of (1) of the lemma, all the assertions about signature reduce immediately from the extended group to the case of $G$; so we will make no further mention of ${ }^{\delta} G$. 
So we may assume henceforth that

$$
\text { the infinitesimal character } \lambda_{0} \text { is regular. }
$$

Writing $H$ for the maximal torus underlying the Langlands parameter $\Gamma=$ $(\Lambda, \nu)$, we get a unique positive system

$$
R_{\text {full }}^{+}(\Gamma)=R^{+}(\mathfrak{g}, \mathfrak{h})_{\text {full }}(\Gamma)
$$

making $(d \Lambda, \nu)$ real dominant, and therefore a corresponding Borel subalgebra $\mathfrak{b}(\Gamma)$ (Definition 5.3). We proceed by increasing induction on the dimension of the $K(\mathbb{C})$ orbit of $\mathfrak{b}(\Gamma)$. (The original Kazhdan-Lusztig conjecture Theorem 18.14, in the case of integral infinitesimal character, concerns the intersection cohomology complex on the closure of $K(\mathbb{C}) \cdot \mathfrak{b}(\Gamma)$, coming from a local system corresponding to $\Gamma$. The proof in that case proceeds by induction on the dimension of this orbit, along exactly the lines we are now following.)

Suppose first that there is a complex simple root $\alpha_{\Gamma}$ for $R_{\text {full }}^{+}(\Gamma)$, such that

$$
\theta \alpha_{\Gamma} \notin R_{\text {full }}^{+}(\Gamma), \quad \alpha_{\Gamma} \notin R(\Gamma) .
$$

$\{$ eq: cplxnonint $\}$

That is, $\alpha_{\Gamma}$ is assumed to be nonintegral. Write $\alpha$ for the corresponding simple root in $R_{s}^{+}$(Proposition 16.2). In the setting of (16.8), we choose the weight $\phi$ not to make $\lambda_{0}+d \phi$ real dominant as in (16.8h), but instead to put it in the adjacent Weyl chamber:

$$
\lambda_{0}+d \phi \text { is regular and real dominant for } s_{\alpha}\left(R_{s}^{+}\right) .
$$

Translation across this nonintegral wall is an equivalence of categories by Corollary 16.9. The translated Langlands parameter $\Gamma^{\prime}=t_{\lambda_{0}}^{\lambda_{0}+\phi}(\Gamma)$ is real dominant for $s_{\alpha_{\Gamma}}\left(R_{\text {full }}^{+}(\Gamma)\right.$, and therefore (by (20.9c)) the corresponding orbit of Borel subalgebras has dimension one less; so the statements of 2(b) are available by inductive hypothesis for $\Gamma^{\prime}$. In order to use them, we need to know how the translation functor $T_{\lambda_{0}}^{\lambda_{0}+\phi}$ affects orientation numbers and canonical Hermitian forms.

The effect on the orientation number of $\Xi$ (Definition 20.5) is fairly easy. If the simple root $\alpha_{\Xi}$ is complex, then $\ell_{o}(\Xi)$ increases by one if $\theta \alpha_{\Xi}>0$, and decreases by one if $\theta \alpha_{\Xi}<0$. If $\alpha_{\Xi}$ is real, then $\ell_{0}(\Xi)$ increases by one if

$$
(-1)^{\left[\left\langle\lambda_{0}, \alpha_{s}^{\vee}\right\rangle\right]}=\Xi_{\mathfrak{q}}\left(m_{\alpha_{\Xi}}\right),
$$

and decreases by one otherwise. (The point for this second fact is that if $t \in \mathbb{R}-\mathbb{Z}$, then the integer parts $[t]$ and $[-t]$ (meaning "greatest integer not 
exceeding") have opposite parity. In order to use the inductive hypothesis, we need to verify (still assuming that we are crossing a single nonintegral wall as in $(20.9 \mathrm{~d})$ that the translation functor $T_{\lambda_{0}}^{\lambda_{0}+\phi}$ does not change the sign of the canonical $c$-invariant form in case the orientation number increases by one, and does change the sign in case the orientation number decreases by one. This is another version of the lowest $K$-type calculation used for Lemma 20.7(1). The most difficult case is that of real $\alpha_{\Xi}$, but that case can be reduced fairly easily to $S L(2, \mathbb{R})$.

Suppose next that there is a complex simple root $\alpha_{\Gamma}$ for $R_{\text {full }}^{+}(\Gamma)$, such that

$$
\theta \alpha \notin R_{\text {full }}^{+}(\Gamma), \quad \alpha \in R(\Gamma) .
$$

$\{$ eq: cplxint $\}$

That is, $\alpha_{\Gamma}$ is assumed to be integral. Write $\alpha$ for the corresponding simple root in $R_{s}^{+}$. (This case is the most serious one.) Write

$$
m=\left\langle d \gamma, \alpha_{\Gamma}^{\vee}\right\rangle
$$

a strictly positive integer. There is a Langlands parameter

$$
\Gamma^{\prime}=\Gamma-m \alpha_{\Gamma}
$$

corresponding to the positive root system

$$
s_{\alpha_{\Gamma}}\left(R_{\text {full }}^{+}(\Gamma)\right)=R_{\text {full }}^{+}(\Gamma)-\left\{\alpha_{\Gamma}\right\} \cup\left\{-\alpha_{\Gamma}\right\} .
$$

The $K(\mathbb{C})$-orbit of the corresponding Borel subalgebra has dimension one less than that for $\Gamma$, so the inductive hypothesis applies to $\Gamma^{\prime}$.

We will not recall all of the machinery of wall-crossing translation functors developed in $[39,40]$. The central idea is to use these functors to construct from $J\left(\Gamma^{\prime}\right)$ a new module

$$
U_{\alpha}\left(J\left(\Gamma^{\prime}\right)\right)
$$

The composition factors of $U_{\alpha}\left(J\left(\Gamma^{\prime}\right)\right)$ consist of $J(\Gamma)$ (with multiplicity one) and certain composition factors of $I_{\text {quo }}\left(\Gamma^{\prime}\right)$. The construction provides a nondegenerate $c$-invariant form on $U_{\alpha}\left(J\left(\Gamma^{\prime}\right)\right)$, and what we need to do is understand that form. The key calculation is that when we apply translation across the wall to the standard module $I_{\text {quo }}\left(\Gamma^{\prime}\right)$, obtaining a module $M$ with a short exact sequence

$$
0 \rightarrow I_{\text {quo }}(\Gamma) \rightarrow M \rightarrow I_{\text {quo }}\left(\Gamma^{\prime}\right) \rightarrow 0,
$$

then the induced form on $M$ restricts to a positive multiple of the canonical form on $I_{\text {quo }}(\Gamma)$. (Just as for Lemma 20.7(1), this amounts to a careful 
examination of lowest $K$-types.) From this it follows that the nondegenerate form on $U_{\alpha}\left(J\left(\Gamma^{\prime}\right)\right)$ restricts to a positive multiple of the canonical form on $J(\Gamma)$.

Essentially [26] uses the decomposition theorem to deduce that $U_{\alpha}\left(J\left(\Gamma^{\prime}\right)\right)$ is completely reducible. Using this complete reducibility, everything else about the form on $U_{\alpha}\left(J\left(\Gamma^{\prime}\right)\right)$ follows by relating $U_{\alpha}$ to the first level of the Jantzen filtration of $I_{\text {quo }}\left(\Gamma^{\prime}\right)$, and applying the inductive hypothesis.

The remaining possibility is that for every complex simple root $\alpha_{\Gamma}, \theta \alpha_{\Gamma}$ is also positive. From this it follows that

$$
\text { the set of nonreal roots in } R_{\text {full }}^{+}(\Gamma) \text { is } \theta \text {-stable. }
$$

$\{$ eq: thetastable $\}$

Writing $\mathfrak{u}=\mathfrak{u}(\Gamma)$ for the span of the root spaces of these nonreal positive roots, we get as in (9.3) a $\theta$-stable parabolic subalgebra

$$
\mathfrak{q}=\mathfrak{l}+\mathfrak{u}=\mathfrak{l}(\Gamma)+\mathfrak{u}(\Gamma)
$$

satisfying the (very strong) positivity condition

$$
\left\langle d \gamma, \beta^{\vee}\right\rangle>0 \quad(\beta \in \Delta(\mathfrak{u}, \mathfrak{h})) .
$$

This condition guarantees that cohomological induction from $L$ by $\mathfrak{q}$ is an exact functor carrying irreducibles to irreducibles, and respecting $c$-invariant Hermitian forms (Theorem 10.9). Because Langlands parameters behave in a simple way under this induction, we see that length and orientation number are preserved; so the assertions in 2(b) of the theorem are reduced to the subgroup $L$. That is, we may assume that

$$
\text { the Cartan subgroup } H \text { for } \Gamma \text { is split; }
$$

that is, that $I_{\text {quo }}(\Gamma)$ is a minimal principal series representation for a split group. If there are no real roots satisfying the parity condition (18.11) then $I(\Gamma)$ is irreducible, and the assertions are easy. So suppose that there are real roots satisfying the parity condition. We proceed by induction on the smallest height of such a root. If the height is equal to one, there is a simple root (necessarily integral) satisfying the parity condition. In this case we argue essentially as in the case (20.9e) above. If the height is greater than one, we can choose a nonintegral simple root $\alpha_{\Gamma}$ such that the simple reflection $s_{\alpha_{\Gamma}}$ decreases the height. In this case we can translate across the nonintegral wall for $\alpha_{\Gamma}$ as in the case (20.9c) above. 
Corollary 20.10. Suppose $\Gamma$ and $\Psi$ are Langlands parameters of (real) infinitesimal character $\chi$. Then the signature character polynomial (Definition 20.4) is

$$
P_{\Gamma, \Psi}^{c}=s^{\left(\ell_{o}(\Psi)-\ell_{o}(\Gamma)\right) / 2} P_{\Gamma, \Psi}(s q) .
$$

In particular

1. if $\ell_{o}(\Psi)-\ell_{o}(\Gamma) \equiv 0(\bmod 4)$, then the signature character coefficient of $(15.12 \mathrm{n})$ is

$$
W_{\Gamma, \Psi}^{c}=\left(\text { sum of even coeffs of } P_{\Gamma, \Psi}\right)+s\left(\text { sum of odd coeffs of } P_{\Gamma, \Psi}\right) ;
$$

2. if $\ell_{o}(\Psi)-\ell_{o}(\Gamma) \equiv 2(\bmod 4)$, then the signature character coefficient is

$$
W_{\Gamma, \Psi}^{c}=\left(\text { sum of odd coeffs of } P_{\Gamma, \Psi}\right)+s\left(\text { sum of even coeffs of } P_{\Gamma, \Psi}\right) \text {. }
$$

The same result holds for the extended group ${ }^{\delta} G$.

Here are representation-theoretic translations of these results.

Corollary 20.11. Suppose $\Gamma$ and $\Psi$ are Langlands parameters of (real) infinitesimal character $\chi$.

1. The signature function for the c-invariant form on the irreducible representation $J(\Psi)$ (see (15.10)) is

$$
\operatorname{sig}_{J(\Psi)}^{c}=\sum_{\Gamma \in B(\chi)} s^{\left(\ell_{o}(\Psi)-\ell_{o}(\Gamma)\right) / 2} P_{\Gamma, \Psi}(s) \operatorname{sig}_{I(\Gamma)}^{c} .
$$

2. The signature function for the Jantzen c-invariant form (Proposition 14.7 on the standard representation $I(\Gamma)$ is

$$
\operatorname{sig}_{I(\Gamma)}^{c}=\sum_{\Xi \in B(\chi)} s^{\ell_{o}(\Xi)-\ell_{o}(\Gamma)} Q_{\Xi, \Gamma}(s) \operatorname{sig}_{J(\Xi)}^{c} \cdot
$$

The same results hold for the extended group ${ }^{\delta} G$.

Proof. Part (1) is (15.12o), together with the formula in Corollary 20.10 for the signature character matrix. Part (2) is Corollary 15.13(2), together with the formula in Theorem 20.6. 


\section{Deformation to $\nu=0$}

Fix a (possibly singular) real infinitesimal character $\chi$, and the finite set $B(\chi)$ of Langlands parameters of infinitesimal character $\chi$ as in (15.1). What we have achieved in Corollary 20.11 is the computability of the formulas (15.120) expressing the signature character of each irreducible representation $J(\Psi)$ as a $\mathbb{W}$-combination of signature characters of standard representations:

$$
\operatorname{sig}_{J(\Psi)}^{c}=\sum_{\Gamma \in B(\chi)} W_{\Gamma, \Psi}^{c} \operatorname{sig}_{I(\Gamma)}^{c} .
$$

That is, we have computed the coefficients $W_{\Gamma, \Psi}^{c}$ in terms of the (known) Kazhdan-Lusztig polynomials for $G$.

In this section we will describe how to compute the signature of each standard representation $I(\Gamma)$ appearing on the right side. As in Section 6, we write

$$
H=T A, \quad G^{A}=M A
$$

for the Langlands decomposition of the Cartan subgroup and for the centralizer of its vector part, and

$$
\Gamma=(\Lambda, \nu)
$$

for the corresponding decomposition of the parameter; $\Lambda$ determines a limit of discrete series $I^{M}(\Lambda)$ for $M$, and $\nu$ may be identified with a character $e^{\nu}$ of $A$. We also write

$$
d \gamma=(d \Lambda, \nu)=(\lambda, \nu) \in \mathfrak{t}^{*}+\mathfrak{a}^{*}=\mathfrak{h}^{*}
$$

as in Theorem 16.6; the weight $d \gamma$ is a representative of the infinitesimal character $\chi$. We will also need the parameters $\Gamma_{t}$ introduced in (14.1).

What makes the signature of $I(\Gamma)$ complicated is the fact that $\nu$ is not zero. Here is how to fix that.

$\{$ thm:deftozero $\}$

Theorem 21.3. For each Langlands parameter $\Gamma=(\Lambda, \nu)$ as above, there is a computable and unique finite formula

$$
\operatorname{sig}_{I(\Gamma)}^{c}=\operatorname{sig}_{I(\Lambda, 0)}^{c}+(s-1) \sum_{\Lambda^{\prime} \in \Pi_{f i n, d i s c}(G)} M_{\Lambda^{\prime}, \Gamma} \operatorname{sig}_{I\left(\Lambda^{\prime}, 0\right)}^{c} \quad\left(M_{\Lambda^{\prime}, \Gamma} \in \mathbb{Z}\right) .
$$

Here final discrete parameters are defined in Definition 6.5. Every $\Lambda^{\prime}$ appearing in the sum satisfies the bound

$$
\left|d \Lambda^{\prime}\right|^{2}<|(d \Lambda, \nu)|^{2}
$$


This bound determines a finite subset of $\Pi_{\text {fin,disc }}$.

An exactly parallel result holds for the extended group ${ }^{\delta} G$.

The term $I((\Lambda, 0)$ on the right need not be final, but one can always use Hecht-Schmid character identities to write it in terms of final parameters. If the set of lowest $K$-types is

$$
\mathcal{L}(\Lambda)=\left\{\mu_{1}, \ldots, \mu_{r}\right\} \subset \widehat{K},
$$

and

$$
\mu_{i}=\mu\left(\Lambda_{i}\right) \quad\left(\Lambda_{i} \in \Pi_{\text {fin }, \operatorname{disc}}(G)\right)
$$

(Theorem 6.6), then

$$
I(\Lambda, 0)=\sum_{i=1}^{r} I\left(\Lambda_{i}, 0\right) .
$$

This identity is true also for the natural $c$-invariant Hermitian forms (which were all normalized to be positive on the lowest $K$-types). In this way we get a signature formula entirely in terms of final discrete parameters.

Before proving Theorem 21.3, we record what it tells us about signature formulas.

Corollary 21.4. Suppose $\Psi=(\Lambda, \nu)$ is a Langlands parameter of real infinitesimal character as in (21.2). Then there is a computable and unique finite formula

$$
\operatorname{sig}_{J(\Psi)}^{c}=\operatorname{sig}_{I(\Lambda, 0)}^{c}+\sum_{\Lambda^{\prime} \in \Pi_{f i n, d i s c}(G)} A_{\Lambda^{\prime}, \Psi} \operatorname{sig}_{I\left(\Lambda^{\prime}, 0\right)}^{c}
$$

The coefficients $A_{\Lambda^{\prime}, \Psi}$ belong to the signature ring $\mathbb{W}$ of Definition 15.9. Final discrete parameters are defined in Definition 6.5. Every $\Lambda^{\prime}$ appearing in the sum satisfies the bound

$$
\left|d \Lambda^{\prime}\right|^{2}<|(d \Lambda, \nu)|^{2} .
$$

This bound determines a finite subset of $\Pi_{\text {fin,disc }}$.

An exactly parallel result holds for the extended group ${ }^{\delta} G$.

Proof. We begin with the formula of Corollary 20.11(1) (one large KazhdanLusztig computation), and then apply Theorem 21.3 to each term on the right (a long chain of nested Kazhdan-Lusztig computations).

Now we can apply Theorem 12.9 and get a statement about signatures of invariant Hermitian forms. 
Corollary 21.5. Suppose $G$ is a real reductive group with extended group ${ }^{\delta} G$ (Definition 12.3). Fix a strong involution $x$ for $G$, an eigenvalue $\zeta$ for the central element $z=x^{2}$, and a square root $\zeta^{1 / 2}$ for $\zeta$ as in Definition 12.8.

Suppose $\Psi=(\Lambda, \nu)$ is a Langlands parameter for ${ }^{\delta} G$ of real infinitesimal character, in which the central element $z$ acts by the scalar $\zeta$.

1. There is a computable and unique finite formula

$$
\operatorname{sig}_{J(\Psi)}^{c}=\sum_{\Lambda^{\prime} \in \Pi_{f i n, d i s c}(\delta G)} B_{\Lambda^{\prime}, \Psi} \operatorname{sig}_{I\left(\Lambda^{\prime}, 0\right)}^{c}
$$

with coefficients $B_{\Lambda^{\prime}, \Psi} \in \mathbb{W}$ (Definition 15.9).

2. The representation $J(\Psi)$ admits an invariant Hermitian form

$$
\langle v, w\rangle_{J(\Psi)}^{0}=\zeta^{-1 / 2}\langle x \cdot v, w\rangle_{J(\Psi)}^{c}=\zeta^{1 / 2}\langle v, x \cdot w\rangle_{J(\Psi)}^{c} .
$$

3. For each $\Lambda^{\prime}$ appearing in the formula of (1), the central element $z$ must act by $\zeta$. Consequently the central element $x \in{ }^{\delta} K$ must act by a scalar

$$
(-1)^{\epsilon\left(\Lambda^{\prime}\right)} \zeta^{1 / 2}
$$

on the unique lowest ${ }^{\delta} K$-type $\mu\left(\Lambda^{\prime}\right)$ of $I\left(\Lambda^{\prime}, 0\right)$; here the parity

$$
\epsilon\left(\Lambda^{\prime}\right) \in \mathbb{Z} / 2 \mathbb{Z}
$$

changes if we change our (fixed global) choice of square root $\zeta^{1 / 2}$.

4. There is a computable and unique finite formula

$$
\operatorname{sig}_{J(\Psi)}^{0}=\sum_{\Lambda^{\prime} \in \Pi_{f i n, d i s c}\left({ }^{\delta} G\right)} s^{\epsilon\left(\Lambda^{\prime}\right)} B_{\Lambda^{\prime}, \Psi} \operatorname{sig}_{I\left(\Lambda^{\prime}, 0\right)}^{0} .
$$

Here the signatures on the right are the positive-definite invariant Hermitian forms on the irreducible tempered representations $I\left(\Lambda^{\prime}, 0\right)$.

As a consequence, $J(\Psi)$ is unitary if and only if one of the following conditions is satisfied:

a) For every $\Lambda^{\prime}$, the coefficient $B_{\Lambda^{\prime}, \Psi}$ is an integer multiple of $s^{\epsilon\left(\Lambda^{\prime}\right)}$; or

b) For every $\Lambda^{\prime}$, the coefficient $B_{\Lambda^{\prime}, \Psi}$ is an integer multiple of $s^{\epsilon\left(\Lambda^{\prime}\right)+1}$. 
The corollary says that to test whether $J(\Psi)$ is unitary, we must first write down a formula as in Corollary 21.4 for the $c$-invariant form on the extended group ${ }^{\delta} G$. (The first term $\operatorname{sig}_{I(\Lambda, 0)}^{c}$ must be rewritten as a sum over the lowest ${ }^{\delta} K$-types as explained after Theorem 21.3.) If any coefficient in that expression is of the form $a+b s$ with $a$ and $b$ both nonzero, then $J(\Psi)$ cannot be unitary. If every coefficient is either an integer or an integer multiple of $s$, then we must compare this behavior with the action of the element $x$ on the lowest ${ }^{\delta} K$-type of the corresponding standard representation. If they either agree everywhere or disagree everywhere, then $J(\Psi)$ is unitary; otherwise it is not.

The proof of Theorem 21.3 will occupy the rest of this section. The idea is to deform the continuous parameter $\nu$ to zero, and to compute the changes in the signature along the way. Mathematically the simplest way to perform this deformation is in a straight line. We will first describe how to do that, and then return to a more circuitous but (perhaps) computationally preferable path. The main step is Corollary 15.13. Here is a reformulation of that result using the detailed information about Jantzen filtrations and forms contained in Theorem 20.6.

\{thm: sigchange $\}$

Theorem 21.6. Suppose $\Gamma$ is a Langlands parameter of real infinitesimal character, so that the Langlands quotient $J(\Gamma)$ admits a nonzero cinvariant Hermitian form $\langle,\rangle_{1}^{c}$. Consider the family of standard representations $I_{\mathrm{quo}}\left(\Gamma_{t}\right)$ (for $t \geq 0$ ) defined in (14.1), and the family of c-invariant forms $\langle,\rangle_{t}^{c}$ extending $\langle,\rangle_{1}^{c}$ as in Proposition 14.7. For every $t \geq 0$, define forms

$$
\langle,\rangle_{t}^{c,[r]} \text { on } I_{\mathrm{quo}}\left(\Gamma_{t}\right)^{r} / I_{\mathrm{quo}}\left(\Gamma_{t}\right)^{r+1}
$$

with signatures

$$
\operatorname{sig}_{I\left(\Gamma_{t}\right)}^{c,[r]}=\operatorname{pos}_{t}^{[r]}+s \operatorname{neg}_{t}^{[r]}: \widehat{K} \rightarrow \mathbb{W}
$$

as in Definition 15.9. Write

$$
\operatorname{sig}_{t}^{c}=\operatorname{sig}_{I\left(\Gamma_{t}\right)}^{c}=\sum_{r=0}^{\infty} \operatorname{sig}_{I\left(\Gamma_{t}\right)}^{c,[r]}
$$

for the signature of the (nondegenerate) Jantzen form on gr $I_{\mathrm{quo}}\left(\Gamma_{t}\right)$. Consider a finite subset

$$
0<t_{r}<t_{r-1}<\cdots<t_{1} \leq 1 \subset(0,1] .
$$

so that $I\left(\Gamma_{t}\right)$ is irreducible for $t \in(0,1] \backslash\left\{t_{i}\right\}$. 
1. On the complement of $\left\{t_{i}\right\}$, the form $\langle,\rangle_{t}^{c}$ is nondegenerate and of locally constant signature

$$
\operatorname{sig}_{t}^{c}=\operatorname{sig}_{t}^{c,[0]}
$$

2. Choose $\epsilon$ so small that $I\left(\Gamma_{t}\right)$ is irreducible for $t \in[1-\epsilon, 1)$ and for $t \in(1,1+\epsilon]$. Then

$$
\begin{aligned}
\operatorname{sig}_{1+\epsilon}^{c} & =\operatorname{sig}_{1}^{c}=\operatorname{sig}_{1-\epsilon}^{c} \\
& +(s-1) \sum_{\substack{\Xi \in B(\chi), \Xi<\Gamma \\
\ell(\Xi)-\ell(\Gamma) \text { odd }}} s^{\left(\ell_{o}(\Xi)-\ell_{o}(\Gamma)\right) / 2} Q_{\Xi, \Gamma}(s) \operatorname{sig}_{J(\Xi)}^{c} .
\end{aligned}
$$

3. Under the same hypotheses as in (2),

$$
\begin{aligned}
\operatorname{sig}_{I\left(\Gamma_{1+\epsilon}\right)}^{c} & =\operatorname{sig}_{I\left(\Gamma_{1}\right)}^{c}=\operatorname{sig}_{I\left(\Gamma_{(1-\epsilon)}\right)}^{c} s^{\left(\ell_{o}(\Phi)-\ell_{o}(\Gamma)\right) / 2} P_{\Phi, \Xi}(s) Q_{\Xi, \Gamma}(s) \operatorname{sig}_{I(\Phi)}^{c} \\
& +(s-1) \sum_{\substack{\Phi, \Xi \in B(\chi) \\
\Phi \leq \Xi<\Gamma \\
\ell(\Xi)-\ell(\Gamma) o d d}} \\
& =\operatorname{sig}_{I\left(\Gamma_{1}\right)}^{c}=\operatorname{sig}_{I\left(\Gamma_{(1-\epsilon)}\right)}^{c} \\
& +(s-1) \sum(-1)^{\left(\ell_{o}(\Phi)-\ell_{o}(\Gamma)\right) / 2} P_{\Phi, \Xi}(-1) Q_{\Xi, \Gamma}(-1) \operatorname{sig}_{I(\Phi)}^{c}
\end{aligned}
$$

Proof. Part (1) is exactly Corollary 15.13(1). Part (2) is Corollary 15.13(3), with the explicit formulas from Theorem 20.6. The first formula in (3) plugs Corollary 20.11 into (2). The second is a consequence of the first because of the identity in $\mathbb{W}$

$$
(s-1) s=(s-1)(-1)
$$

Proof of Theorem 21.3. We fix an invariant symmetric bilinear form on $\mathfrak{g}$ that is negative definite on the compact form $\mathfrak{g}\left(\mathbb{R}, \sigma_{c}\right)$ (Theorem 3.4). This form gives rise to a $W(\mathfrak{g}, \mathfrak{h})$-invariant form $\langle$,$\rangle on the dual \mathfrak{h}^{*}$ of any Cartan subalgebra, which is positive definite on the canonical real weights (the real span of the differentials of algebraic characters). We can arrange for this form to take rational values on the lattice $X^{*}(H(\mathbb{C}))$; the denominators appearing are then necessarily bounded by some positive integer $N$. In this case the form also takes rational values on the differentials of discrete Langlands parameters, this time with denominators bounded by $4 N$. (We 
may gain one 2 from the $\rho$ shift in the parameter, and one from the operation $(1+\theta) / 2$ of restricting to the compact part.)

To prove the theorem, we fix a bound $M$ on the size of infinitesimal characters, and prove the theorem for all Langlands parameters

$$
\Gamma^{\prime}=\left(\Lambda^{\prime}, \nu^{\prime}\right), \quad\left\langle d \Lambda^{\prime}, \nu^{\prime}\right\rangle \leq M .
$$

The proof proceeds by downward induction on $\left\langle d \Lambda^{\prime}, d \Lambda^{\prime}\right\rangle=k$. For $k>M$ there are no such Langlands parameters, and the theorem is vacuously true; so we assume that the theorem is known for $\Gamma^{\prime}$ with

$$
\left\langle d \Lambda^{\prime}, d \Lambda^{\prime}\right\rangle>\langle d \Lambda, d \Lambda\rangle
$$

We apply the deformation formulas of Theorem $21.6 r$ times, at the points $t_{1}, t_{2}, \ldots, t_{r}$. We get in the end a (computable) formula

$$
\operatorname{sig}_{I(\Gamma)}^{c}=\operatorname{sig}_{I(\Lambda, 0)}^{c}+(s-1) \sum_{\Phi \in B\left(\chi_{t_{j}}\right)} M_{\Phi, \Gamma} \operatorname{sig}_{I(\Phi)}^{c} .
$$

$\{$ eq:rdefform $\}$

Here every Langlands parameter $\Phi$ appearing has infinitesimal character of the form

$$
\chi_{t_{j}} \leftrightarrow\left(d \Lambda, t_{j} \nu\right),
$$

which has size less than or equal to the size of $\chi$, and so is bounded by $M$. The condition in Theorem 21.6(5) $\Phi<\Gamma_{t_{j}}$ implies in particular that the discrete part $\Lambda(\Phi)$ satisfies

$$
\langle d \Lambda(\Phi), d \Lambda(\Phi)\rangle>\langle d \Lambda, d \Lambda\rangle
$$

By the inductive hypothesis, each $\operatorname{sig}_{I(\Phi)}^{c}$ has a formula

$$
\operatorname{sig}_{I(\Phi)}^{c}=\operatorname{sig}_{I(\Lambda(\Phi), 0)}^{c}+(s-1) \sum_{\Lambda^{\prime} \in \Pi_{\text {fin }, \text { disc }}(G)} M_{\Lambda^{\prime}, \Phi} \operatorname{sig}_{I\left(\Lambda^{\prime}, 0\right)}^{c} \quad\left(M_{\Lambda^{\prime}, \Phi} \in \mathbb{Z}\right) .
$$

By the remarks after the statement of Theorem 21.3, the first term on the \{eq:indhypdefform $\}$ right can be rewritten as a sum (over the lowest $K$-types of $I(\Phi)$ ) of standard final discrete representations. Now if we insert all these inductively known formulas (21.7f) in (21.7c), we get the conclusion of the theorem.

To implement this computation, one needs to compute (among other things) all the Kazhdan-Lusztig polynomials at various infinitesimal characters like that of any $\Gamma_{t}=(\Lambda, t \nu)$. When such an infinitesimal character 
is (nearly) integral, the computation is long and difficult. We would like therefore to outline another way to proceed, involving only more restricted families of Kazhdan-Lusztig polynomials.

We begin by setting up the bookkeeping.

The decompositions

$$
\mathfrak{h}=\mathfrak{a}+\mathfrak{t}, \quad \mathfrak{h}^{*}=\mathfrak{a}^{*}+\mathfrak{t}^{*}
$$

are into the -1 and +1 eigenspaces of the Cartan involution $\theta$. Applied to any $\xi \in \mathfrak{h}$, they are therefore given by

$$
\xi=\xi_{\mathfrak{a}}+\xi_{\mathfrak{t}}, \quad \xi_{\mathfrak{a}}=(\xi-\theta \xi) / 2, \quad \xi_{\mathfrak{t}}=(\xi+\theta \xi) / 2,
$$

and similarly for $\mathfrak{h}^{*}$. For any coroot $\alpha^{\vee} \in \mathfrak{h}$, this means in particular that the value on the infinitesimal character $\gamma$ is

$$
\left\langle\gamma, \alpha^{\vee}\right\rangle=\left\langle(\lambda, \nu), \alpha^{\vee}\right\rangle=\left\langle\nu, \alpha_{\mathfrak{a}}^{\vee}\right\rangle+\left\langle\lambda, \alpha_{\mathfrak{t}}^{\vee}\right\rangle
$$

Here we will think of

$$
\left\langle\lambda, \alpha_{\mathfrak{t}}^{\vee}\right\rangle=\left(\left\langle\lambda, \alpha^{\vee}\right\rangle+\left\langle\lambda, \theta \alpha^{\vee}\right\rangle\right) / 2={ }_{\operatorname{def}} \ell_{\alpha} \in \frac{1}{2} \mathbb{Z}
$$

as a constant, and

$$
\left\langle\nu, \alpha_{\mathfrak{a}}^{\vee}\right\rangle=\left(\left\langle\nu, \alpha^{\vee}\right\rangle+\left\langle\nu,-\theta \alpha^{\vee}\right\rangle\right) / 2={ }_{\operatorname{def}} y_{\alpha} \in \mathbb{R}
$$

as variable. For example, if $\alpha$ is not imaginary and $n$ is an integer, then the collection of $\nu \in \mathfrak{a}_{0}^{*}$ for which $\left\langle\gamma, \alpha^{\vee}\right\rangle=n$ is

$$
\left\{\nu \in \mathfrak{a}_{0}^{*} \mid\left\langle\nu, \alpha_{\mathfrak{a}}^{\vee}\right\rangle=n-\ell_{\alpha}\right\},
$$

an affine hyperplane in $\mathfrak{a}_{0}^{*}$. (The point is that $\alpha_{\mathfrak{a}}^{\vee}$ is a nonzero linear functional on $\mathfrak{a}_{0}^{*}$ since $\alpha$ is not imaginary; so the set where it assumes the specified value $n-\ell_{\alpha}$ is an affine hyperplane.)

Definition 21.9. In the setting of Proposition 6.6, and with the notation of (21.8), the restricted roots of $A$ in $G$ are

$$
R(G, A)=\left\{\phi=\alpha_{\mathfrak{a}} \mid \alpha \in R(G, H)-R_{i \mathbb{R}}(G, H)\right\} \subset \mathfrak{a}^{*}-\{0\} .
$$

(Definition 5.7, and notation (21.8)). Considering only nonimaginary roots guarantees that these restrictions to $\mathfrak{a}$ are all nonzero. The set of restricted roots is often not a root system. In [18] one can find a 
description of a large subset which is a root system, but we will have no need for this.

Even though the restricted roots $R(G, A)$ do not constitute a root system, it is convenient to make formal use of "coroots." We define the restricted coroots of $A$ in $G$ to be

$$
R^{\vee}(G, A)=\left\{\phi^{\vee}=\alpha_{\mathfrak{a}}^{\vee} \mid \alpha^{\vee} \in R^{\vee}(G, H)-R_{i \mathbb{R}}^{\vee}(G, H)\right\} \subset \mathfrak{a}-\{0\} .
$$

We are particularly interested in the lines

$$
\begin{gathered}
{[\phi]=\mathbb{Q} \phi \subset \mathfrak{a}^{*},} \\
R(G, A)^{[\phi]}=R(G, A) \cap[\phi] ;
\end{gathered}
$$

the set of restricted roots is the disjoint union of these finitely many lines. To each line of roots correspond the hyperplane and line

$$
\mathfrak{a}^{[\phi]}=\operatorname{ker} \phi \subset \mathfrak{a}, \quad \mathfrak{a}_{[\phi]}=\mathbb{C} \phi^{\vee}, \quad \mathfrak{a}=\mathfrak{a}^{[\phi]} \oplus \mathfrak{a}_{[\phi]},
$$

with $\phi^{\vee}$ a restricted coroot for some root restricting in $[\phi]$. (We will show in Lemma 21.10 below that the line through $\phi^{\vee}$ is independent of the choice of this root.) Dually, we have

$$
\left(\mathfrak{a}^{*}\right)^{\left[\phi^{\vee}\right]}=\operatorname{ker} \phi^{\vee} \subset \mathfrak{a}^{*}, \quad \mathfrak{a}_{\left[\phi^{\vee}\right]}^{*}=\mathbb{C} \phi, \quad \mathfrak{a}^{*}=\left(\mathfrak{a}^{*}\right)^{\left[\phi^{\vee}\right]} \oplus \mathfrak{a}_{\left[\phi^{\vee}\right]}^{*} .
$$

Attached to each restricted root line $[\phi]$ there is a Levi subgroup (for a real parabolic)

$$
L^{[\phi]} A^{[\phi]}=G^{\mathfrak{a}[\phi]} \supset M A .
$$

This Levi subgroup is characterized by

$$
R\left(L^{[\phi]} A^{[\phi]}, H\right)=\left\{\alpha \in R(G, H) \mid \alpha_{\mathfrak{a}} \in[\phi]\right\}==_{\operatorname{def}} R(G, H)^{[\phi]} .
$$

Each $R(G, H)^{[\phi]}$ contains all the imaginary roots. We have

$$
H \cap L^{[\phi]}=T A_{[\phi]} ;
$$

the restriction of $\nu \in \mathfrak{a}^{*}$ to the line $\mathfrak{a}_{[\phi]}^{*}$ is given by $\left\langle\nu, \phi^{\vee}\right\rangle$.

The Weyl group of $A$ in $G$ is

$$
W(G, A)=N_{G}(A) / Z_{G}(A) \simeq W(G, H) / W_{i \mathbb{R}}(G, H)
$$

The last quotient is by the subgroup of the real Weyl group acting trivially on $A$; that is the same as

$$
W_{i \mathbb{R}}(G, H)=W(G, H) \cap W\left(R_{i \mathbb{R}}, H\right),
$$


the subgroup contained in the Weyl group of the imaginary roots. We will need the subgroup stabilizing the discrete parameter $\Lambda$ :

$$
W(G, A)^{\Lambda} \simeq W(G, H)^{\Lambda} / W_{i \mathbb{R}}(G, H)^{\Lambda} .
$$

Lemma 21.10. In the setting of Definition 21.9, suppose that $\alpha$ and $\beta$ are nonimaginary roots such that $\alpha_{\mathfrak{a}} \in \mathbb{Q} \beta_{\mathfrak{a}}$; that is, that the lines $\left[\alpha_{\mathfrak{a}}\right]$ and $\left[\beta_{\mathfrak{a}}\right]$ of restricted roots coincide. Then $\alpha_{\mathfrak{a}}^{\vee} \in \mathbb{Q} \beta_{\mathfrak{a}}^{\vee}$; that is, the lines $\left[\alpha_{\mathfrak{a}}^{\vee}\right]$ and $\left[\beta_{\mathfrak{a}}^{\vee}\right]$ of restricted coroots also coincide.

Proof. Choose an $\operatorname{Ad}(G)$-invariant symmetric bilinear form $\langle$,$\rangle on \mathfrak{g}$ that is negative definite on $\mathfrak{k}_{0}$, positive definite on $\mathfrak{s}_{0}$, and making these spaces orthogonal (notation as in Theorem 3.4). Then the induced forms on $\mathfrak{h}$ and $\mathfrak{h}^{*}$ are positive definite on the spans of the coroots and roots, and

$$
\lambda\left(\alpha^{\vee}\right)=2\langle\lambda, \alpha\rangle /\langle\alpha, \alpha\rangle .
$$

That is, the identification of $\mathfrak{h}$ with $\mathfrak{h}^{*}$ defined by the bilinear form identifies the coroot $\alpha^{\vee}$ with $2 \alpha /\langle\alpha, \alpha\rangle$. Orthogonal projection on $\mathfrak{a} \simeq \mathfrak{a}^{*}$ gives

$$
\alpha_{\mathfrak{a}}^{\vee}=2 \alpha_{\mathfrak{a}} /\langle\alpha, \alpha\rangle .
$$

Now the conclusion of the lemma is clear.

We are interested in certain affine hyperplanes in $\mathfrak{a}_{0}^{*}$ based on (that is, translates of) the codimension one real subspaces $\left(\mathfrak{a}_{0}^{*}\right)^{\left[\phi^{\vee}\right]}$ introduced in Definition 21.9.

Definition 21.11. An affine hyperplane

$$
\left\{\nu \in \mathfrak{a}_{0}^{*} \mid\left\langle\nu, \phi^{\vee}\right\rangle=q\right\}
$$

is called a potential reducibility hyperplane for $\Lambda$ if it is one of those described in Proposition 6.6: that is, if there is a nonimaginary root $\alpha \in R(G, H)$ such that $\phi^{\vee}=\alpha_{\mathfrak{a}}^{\vee}$, and (for every $\nu$ in the hyperplane, so that $\gamma=(\lambda, \nu)$ as in (21.2)) either

a) $\alpha$ is complex, $\left\langle\gamma, \alpha^{\vee}\right\rangle$ and $\left\langle\gamma,-\theta \alpha^{\vee}\right\rangle$ are both strictly positive integers (equivalently, in the notation of (21.8), $q=n-\ell_{\alpha}$ with $n>\left|\ell_{\alpha}\right|$ an integer); or

b) $\alpha$ is real, $\left\langle\gamma, \alpha^{\vee}\right\rangle$ is a strictly positive integer, and

$$
\Lambda_{\mathfrak{q}}\left(m_{\alpha}\right)=(-1)^{\left\langle\nu, \alpha^{\vee}\right\rangle+1}
$$

(notation (9.3d)); equivalently, $q=n$ is a strictly positive integer of parity opposite to that of $\Lambda_{\mathfrak{q}}\left(m_{\alpha}\right)$. 
The hyperplane is called reorienting for $\Lambda$ if the orientation number of Definition 20.5 changes on crossing the hyperplane: that is, if there is a nonimaginary root $\alpha \in R(G, H)$ such that $\phi^{\vee}=\alpha_{\mathfrak{a}}^{\vee}$, and (for every $\nu$ in the hyperplane, so that $\gamma=(\lambda, \nu)$ as in (21.2)) either

a) $\alpha$ is complex, $\left\langle\gamma, \alpha^{\vee}\right\rangle=0$ and $\left\langle\gamma,-\theta \alpha^{\vee}\right\rangle>0$ (equivalently, in the notation of $\left.(21.8), q=-\ell_{\alpha}<0\right)$; or

b) $\alpha$ is real, $\left\langle\gamma, \alpha^{\vee}\right\rangle$ is a strictly positive integer, and

$$
\Lambda_{\mathfrak{q}}\left(m_{\alpha}\right)=(-1)^{\left\langle\nu, \alpha^{\vee}\right\rangle}
$$

(equivalently, $q=n$ is a strictly positive integer of the same parity as $\left.\Lambda_{\mathfrak{q}}\left(m_{\alpha}\right)\right) ;$ or

c) $\alpha$ is real, $\left\langle\gamma, \alpha^{\vee}\right\rangle$ is a strictly positive integer, and

$$
\Lambda_{\mathfrak{q}}\left(m_{\alpha}\right)=(-1)^{\left\langle\nu, \alpha^{\vee}\right\rangle+1}
$$

(equivalently, $q=n$ is a strictly positive integer of parity opposite to that of $\left.\Lambda_{\mathfrak{q}}\left(m_{\alpha}\right)\right)$.

The third case is just the real root reducibility hyperplane. Crossing a hyperplane of one of the first two kinds in the direction of increasing $\phi^{\vee}$ increases the orientation number by one, so we call them positively reorienting. Crossing a hyperplane of the third kind in the direction of increasing $\phi^{\vee}$ decreases the orientation number by one, so we call these negatively reorienting.

Here is the picture. For our fixed discrete parameter $\Lambda$, the real vector space $\mathfrak{a}_{0}^{*}$ has a hyperplane arrangement given by the potential reducibility and reorienting hyperplanes of Definition 21.11. For every complex root $\alpha$ of $H$, we get the family of equally spaced parallel hyperplanes

$$
\left\langle\nu, \alpha_{\mathfrak{a}}^{\vee}\right\rangle=n-\ell_{\alpha}, \quad\left(n \in \mathbb{Z}, n \geq\left|\ell_{\alpha}\right|\right)
$$

(notation (21.8)); if $\ell_{\alpha}=0$, then the case $n=0$ is excluded. For every real root $\alpha$, we have the family of equally spaced parallel hyperplanes

$$
\left\langle\nu, \alpha_{\mathfrak{a}}^{\vee}\right\rangle=n, \quad(n \in \mathbb{Z}, n>0) .
$$

Within each open region defined by this arrangement, the $c$-invariant Hermitian form has a constant signature. Because none of the hyperplanes 
passes through the origin, the signature is constant near $\nu=0$. More explicitly,

$$
\operatorname{sig}_{I(\Gamma)}^{c}=\operatorname{sig}_{I(\Lambda, 0)}^{c}, \quad\left(\nu \in \mathfrak{a}_{0}^{*} \text { small }\right) .
$$

More explicitly, the conditions on $\nu$ are

$$
\left\langle\nu, \alpha_{\mathfrak{a}}^{\vee}\right\rangle<\left|\ell_{\alpha}\right| \quad\left(\alpha \text { complex }, \ell_{\alpha} \neq 0\right)
$$

and

$$
\left\langle\nu, \alpha_{\mathfrak{a}}^{\vee}\right\rangle<1 \quad\left(\alpha \text { complex or real, } \ell_{\alpha}=0\right) .
$$

In order to prove Theorem 21.3, we need to see what happens to the signature when we cross a single hyperplane in the arrangement.

Theorem 21.13. Suppose $\Gamma=(\Lambda, \nu)$ is a Langlands parameter as in (21.2). Fix a potential reducibility hyperplane

$$
\mathcal{H}\left(\phi^{\vee}, q\right)=\left\{\nu \in \mathfrak{a}_{0}^{*} \mid\left\langle\nu, \phi^{\vee}\right\rangle=q\right\}
$$

as in Definition 21.11. This means in particular that $\phi^{\vee}$ is a restricted coroot: there is a nonimaginary root $\alpha$ with $\phi^{\vee}=\alpha_{\mathfrak{a}}^{\vee}$ (Definition 21.9). Let $\phi=\alpha_{\mathfrak{a}}$ be the corresponding restricted root (which according to Lemma 21.10) is well defined up to a positive rational multiple. Therefore the Levi subgroup

$$
L^{[\phi]} A^{[\phi]} \supset M A
$$

(corresponding to all roots restricting to multiples of $\phi$; cf. Definition 21.9) is well defined. Fix a parabolic subgroup

$$
Q^{[\phi]}=L^{[\phi]} A^{[\phi]} N^{[\phi]} .
$$

1. Suppose $H_{1} \subset L^{[\phi]} A^{[\phi]}$ is any $\theta$-stable Cartan subgroup. Then weak Langlands parameters for $G$ based on $H_{1}$ (Definition 6.3) may be identified with weak Langlands parameters for $L^{[\phi]} A^{[\phi]}$ based on $H_{1}$.

2. The correspondence of (1) is implemented on the level of standard representations by parabolic induction:

$$
\left[I^{G}\left(\Gamma_{1}\right)\right]=\left[\operatorname{Ind}_{Q^{[\phi]}}^{G}\left(I^{L A^{[\phi]}}\left(\Gamma_{1}\right) \otimes 1^{N^{[\phi]}}\right)\right] .
$$

Here the brackets indicate equality in the Grothendieck group.

Assume now that $\nu$ belongs to the potential reducibility hyperplane $\mathcal{H}\left(\phi^{\vee}, q\right)$ and to no other potential reducibility hyperplane. 
3. The equality in (2) is true on the level of standard representations endowed with Jantzen filtrations (Proposition 14.7):

$$
\left(I^{G}\right)_{\mathrm{quo}}^{r}(\Gamma)=\operatorname{Ind}_{Q^{[\phi]}}^{G}\left(\left(I^{L^{[\phi]}} A^{[\phi]}\right)_{\mathrm{quo}}^{r}(\Gamma) \otimes 1^{N^{[\phi]}}\right),
$$

and similarly for the submodule filtrations.

4. Suppose $\Xi$ is a Langlands parameter for $L^{[\phi]} A^{[\phi]}$. Then the multiplicity of $J^{G}(\Xi)$ in the rth level of the Jantzen filtration of $I(\Gamma)$ is equal to the coefficient of $q^{(\ell(\Gamma)-\ell(\Xi)-r) / 2}$ in the Kazhdan-Lusztig multiplicity polynomial $Q_{\Xi, \Gamma}$, which may be computed inside the subgroup $L^{[\phi]}$.

5. The signature of the invariant form on the induced representation changes across the hyperplane $\mathcal{H}\left(\phi^{\vee}, q\right)$ as follows: for $\epsilon>0$ sufficiently small,

$$
\begin{aligned}
\operatorname{sig}_{I(\Lambda,(1+\epsilon) \nu)}^{c} & =\operatorname{sig}_{I(\Gamma)}^{c}=\operatorname{sig}_{I(\Lambda, \nu)}^{c}=\operatorname{sig}_{I(\Lambda,(1-\epsilon) \nu)}^{c} \\
& +(s-1) \sum_{\substack{\Xi \in B(\xi) \\
\ell(\Xi)-\ell(\Gamma) o d d}}^{\left(\ell_{o}(\Xi)-\ell_{o}(\Gamma)\right) / 2} Q_{\Xi, \Gamma}(s) \operatorname{sig}_{J(\Xi)}^{c}
\end{aligned}
$$

Recall from Definition 6.3 that Langlands parameters are weak parameters subject to two additional conditions (5) and (6). The condition (6) is the same for $G$ and for the Levi subgroup, since it involves only imaginary roots. But condition (5), called "final," is stronger for $G$, which may have additional real roots not present in the Levi subgroup.

The calculation in (5) of the theorem cannot quite be done in the Levi subgroup, because the orientation numbers appearing can involve roots outside the Levi.

The change of signature formula in (5) can be rewritten in various ways, exactly as in Theorem 21.6. Indeed this entire theorem is a special case of that one. The point of this formulation is that if one moves the continuous parameter $\nu$ in such a way as to cross only one hyperplane at a time, then the Kazhdan-Lusztig calculations required can be done inside the Levi subgroups $L^{[\phi]}$.

The question of how much one gains in this fashion is a complicated one. Consider for example the split real group of type $E_{8}$. In order to deform invariant Hermitian forms related to the trivial representation using Theorem 21.6, one has to calculate the Kazhdan-Lusztig polynomials for the largest block, in $E_{8}$, which has 453,060 elements. To use Theorem 21.13, the only calculation which must be done in $E_{8}$ is for that portion of the block which 
lies below some parameter $\Gamma$ attached to the (unique) Cartan subgroup with one-dimensional split part. We have not calculated exactly how large that portion of the block is, but there is an upper bound of 191,411. The atlas software can calculate these polynomials (more precisely, the upper left corner of the Kazhdan-Lusztig matrix of size 191,411 by 191,411) in about one-fiftieth the time and one-fifteenth the memory needed to compute the entire matrix.

On the other hand, a complete analysis of invariant forms on irreducible representations requires not only this deformation analysis but also the explicit expression for an irreducible in terms of standard modules in (15.12o); and this is calculated in Corollary 20.11 using all the Kazhdan-Lusztig polynomials.

It may be that the idea in Theorem 21.13 still provides a useful improvement in the deformation part of the calculation. The only way that we can see to make a convincing test is to implement both algorithms and compare performance.

\section{Hyperplane calculations}

The formulas of Theorem 21.13 require explicit calculations in the groups $\{$ sec:hypcalc $\}$

$L^{[\phi]}$ corresponding to a single line of restricted roots $((21.8))$. In this section we will enumerate the corresponding cases. This was done in the (unpublished) work announced in [2].

What is shown in the table is the Dynkin diagram of $L^{[\phi]}$. The roots of the Levi subgroup $M A$ are black. 


\begin{tabular}{|c|c|c|c|}
\hline type & diagram & $G$ & $M A$ \\
\hline$A_{1}$ & ० & $G L(2, \mathbb{R})$ & $G L(1, \mathbb{R}) \times G L(1, \mathbb{R})$ \\
\hline$A_{1} \times A_{1}$ & $\circ \circ$ & $S L(2, \mathbb{C})$ & $G L(1, \mathbb{C})$ \\
\hline$A_{2}$ & $\bullet-0$ & $G L(3, \mathbb{R})$ & $G L(2, \mathbb{R}) \times G L(1, \mathbb{R})$ \\
\hline$A_{2}$ & ○一• & $G L(3, \mathbb{R})$ & $G L(1, \mathbb{R}) \times G L(2, \mathbb{R})$ \\
\hline$A_{3}$ & $\bullet-\circ-\bullet$ & $G L(4, \mathbb{R})$ & $G L(2, \mathbb{R}) \times G L(2, \mathbb{R})$ \\
\hline$A_{3}$ & $\bullet-0-\bullet$ & $G L(2, \mathbb{H})$ & $G L(1, \mathbb{H}) \times G L(1, \mathbb{H})$ \\
\hline$A_{n}$ & O- $\bullet \cdot \cdots \bullet-$ & $\begin{array}{l}U(p, q), p, q \geq 1 \\
p+q=n-1\end{array}$ & $G L(1, \mathbb{C}) \times U(p-1, q-1)$ \\
\hline$B_{n}$ & $\bullet-0-\bullet \cdot$ & $\begin{array}{l}S O(2 p, 2 q+1) \\
p, q \geq 1, p+q=n\end{array}$ & $G L(2, \mathbb{R}) \times S O(2 p-2,2 q-1)$ \\
\hline$B_{n}$ & $\circ-\bullet-\bullet \cdots \bullet \Longrightarrow \bullet$ & $\begin{array}{l}S O(2 p, 2 q+1) \\
p, q \geq 1, p+q=n\end{array}$ & $G L(1, \mathbb{R}) \times S O(2 p-1,2 q)$ \\
\hline$C_{n}$ & $\bullet-0-\bullet \cdot \cdot \bullet$ & $S p(2 n, \mathbb{R})$ & $G L(2, \mathbb{R}) \times S p(2 n-4, \mathbb{R})$ \\
\hline$C_{n}$ & $\bullet-0-\bullet \cdots$ & $\begin{array}{l}S p(p, q), \quad p, q \geq \\
1, p+q=n\end{array}$ & $G L(2, \mathbb{R}) \times S p(p-1, q-1)$ \\
\hline$C_{n}$ & $0-\bullet-\bullet \cdots \bullet<\bullet$ & $S p(2 n, \mathbb{R})$ & $G L(1, \mathbb{R}) \times S p(2 n-2, \mathbb{R})$ \\
\hline$D_{n}$ & & $\begin{array}{l}S O(2 p, 2 q), p, q \geq \\
1, p+q=n\end{array}$ & $G L(2, \mathbb{R}) \times S O(2 p-2,2 q-2)$ \\
\hline$D_{n}$ & & $S O^{*}(2 n)$ & $U(2) \times S O^{*}(2 n-4)$ \\
\hline$D_{n}$ & & $\begin{array}{l}S O(2 p+1,2 q-1) \\
p, q \geq 1, p+q=n\end{array}$ & $S O(2 p, 2 q-2)$ \\
\hline
\end{tabular}

Rank one $M$ for classical $G$ 


\begin{tabular}{|c|c|c|c|}
\hline type & diagram & $G$ & $M A$ \\
\hline$G_{2}$ & $\mathrm{O} \rightleftharpoons \bullet$ & $G_{2}$ & $G L(2, \mathbb{R})_{\text {short }}$ \\
\hline$G_{2}$ & $\bullet \equiv 0$ & $G_{2}$ & $G L(2, \mathbb{R})_{\text {long }}$ \\
\hline$F_{4}$ & $\circ-\bullet \rightleftharpoons \bullet-\bullet$ & $F_{4}$ & $C_{3} \times \mathbb{R}^{\times}$ \\
\hline$F_{4}$ & $\bullet-\bullet \Rightarrow \bullet-0$ & $F_{4}$ & $B_{3} \times \mathbb{R}^{\times}$ \\
\hline$E_{6}$ & & $E_{6}$ & $A_{5} \times \mathbb{R}^{\times}$ \\
\hline$E_{7}$ & $\bullet \ldots, \bullet \bullet-\ldots$ & $E_{7}$ & $D_{6} \times \mathbb{R}^{\times}$ \\
\hline$E_{8}$ & -1 & $E_{8}$ & $E_{7} \times \mathbb{R}^{\times}$ \\
\hline
\end{tabular}

Rank one $M$ for exceptional $G$

\section{References}

[1] Jeffrey D. Adams, Dan M. Barbasch, and David A. Vogan Jr., The Langlands Classification and Irreducible Characters for Real Reductive Groups, Birkhäuser, Boston-Basel-Berlin, 1992.

[2] Dan Barbasch and David A. Vogan Jr., Reducibility of standard representations, Bull. Amer. Math. Soc. (N.S.) 11 (1984), no. 2, 383-385.

[3] A. Beilinson and J. Bernstein, A proof of Jantzen conjectures, I. M. Gel'fand Seminar, Adv. Soviet Math., vol. 16, Amer. Math. Soc., Providence, RI, 1993, pp. 1-50.

[4] A. Beilinson, J. Bernstein, and P. Deligne, Faisceaux pervers 5-171, Analyse et Topologie sur les Espaces Singuliers, volume 1 Astérisque, 1982.

[5] A. Borel, Linear Algebraic Groups, Graduate Texts in Mathematics, Springer-Verlag, New York, 1991.

[6] A. H. Clifford, Representations induced in an invariant subgroup, Ann. of Math. (2) 38 (1937), no. 3, 533-550.

[7] F. du Cloux and M. van Leeuwen, Software for structure and representations of real reductive groups, version 0.4.6, available from http://www . liegroups.org. 
[8] A. I. Fomin and N. N. Šapovalov, A certain property of the characters of irreducible representations of real semisimple Lie groups, Funkcional. Anal. i Priložen. 8 (1974), no. 3, 87-88 (Russian); English transl., Functional Anal. Appl. 8 (1974), 270-271.

[9] Harish-Chandra, Representations of a semisimple Lie group on a Banach space I, Trans. Amer. Math. Soc. 75 (1953), 185-243.

[10] _ Representations of semisimple Lie groups. II, Trans. Amer. Math. Soc. 76 (1954), 26-65.

[11] _ Representations of semisimple Lie groups. III, Trans. Amer. Math. Soc. 76 (1954), 234-253.

[12] _ The characters of semisimple Lie groups, Trans. Amer. Math. Soc. 83 (1956), 98-163.

[13] _ Invariant eigendistributions on a semisimple Lie group, Trans. Amer. Math. Soc. 119 (1965), 457-508.

[14] Henryk Hecht and Wilfried Schmid, Characters, asymptotics and $\mathfrak{n}$ homology of Harish-Chandra modules, Acta Math. 151 (1983), no. 1-2, 49-151.

[15] James E. Humphreys, Introduction to Lie algebras and representation theory, Graduate Texts in Mathematics, vol. 9, Springer-Verlag, New York, 1978. Second printing, revised.

[16] J. C. Jantzen, Moduln mit einem Höchsten Gewicht, Lecture Notes in Mathematics, vol. 750, Springer-Verlag, Berlin-Heidelberg-New York, 1979.

[17] David Kazhdan and George Lusztig, Representations of Coxeter groups and Hecke algebras, Invent. Math. 53 (1979), no. 2, 165-184.

[18] Anthony W. Knapp, Weyl group of a cuspidal parabolic, Ann. Sci. Ecole Norm. Sup. 8 (1975), 275-294.

[19] _ Representation Theory of Semisimple Groups: An Overview Based on Examples, Princeton University Press, Princeton, New Jersey, 1986.

[20] Lie Groups Beyond an Introduction, Second Edition, Progress in Mathematics 140, Birkhäuser, Boston-Basel-Berlin, 2002.

[21] Anthony W. Knapp and David A. Vogan Jr., Cohomological Induction and Unitary Representations, Princeton University Press, Princeton, New Jersey, 1995. 
[22] Anthony W. Knapp and Gregg Zuckerman, Classification of irreducible tempered representations of semisimple Lie groups, Ann. of Math. 116 (1982), 389-501. See also Ann. of Math. 119 (1984), 639.

[23] Anthony W. Knapp and Elias M. Stein, Intertwining operators for semisimple groups II, Invent. Math. 60 (1980), 9-84.

[24] Robert P. Langlands, On the classification of representations of real algebraic groups, Representation Theory and Harmonic Analysis on Semisimple Lie Groups, 1989, pp. 101-170.

[25] G. Lusztig, Characters of Reductive Groups over a Finite Field, Annals of Mathematics Studies, vol. 107, Princeton University Press, Princeton, New Jersey, 1984.

[26] George Lusztig and David A. Vogan Jr., Singularities of closures of K-orbits on flag manifolds, Invent. Math. 71 (1983), no. 2, 365-379.

[27], Quasisplit Hecke algebras and symmetric spaces, arXiv: 1206.0634.

[28] A. L. Onishchik and È. B. Vinberg, Lie groups and algebraic groups, Springer Series in Soviet Mathematics, Springer-Verlag, Berlin, 1990. Translated from the Russian and with a preface by D. A. Leites.

[29] David A. Renard and Peter E. Trapa, Kazhdan-Lusztig algorithms for nonlinear groups and applications to Kazhdan-Patterson lifting, Amer. J. Math. 127 (2005), no. 5, 911-971.

[30] W. Rossmann, Tempered representations and orbits, Duke Math. J. 49 (1982), no. 1, 231-247.

[31] Gérard Schiffmann, Intégrales d'entrelacement et fonctions de Whittaker, Bull. Soc. Math. France 99 (1971), 3-72.

[32] Wilfried Schmid, Some properties of square-integrable representations of semisimple Lie groups, Ann. of Math. 102 (1975), 535-564.

[33] _ Two character identities for semisimple Lie groups, Noncommutative Harmonic Analysis and Lie groups, 1977, pp. 196-225.

[34] I. E. Segal, Hypermaximality of certain operators on Lie groups, Proc. Amer. Math. Soc. 3 (1952), 13-15.

[35] W. Soergel, An irreducible not admissible Banach representation of SL(2, R), Proc. Amer. Math. Soc. 104 (1988), 1322-1324.

[36] B. Speh and David A. Vogan Jr., Reducibility of generalized principal series representations, Acta Math. 145 (1980), 227-299. 
[37] T. A. Springer, Linear algebraic groups, 2nd ed., Progress in Mathematics, vol. 9, Birkhäuser Boston Inc., Boston, MA, 1998.

[38] David A. Vogan Jr., Representations of Real Reductive Lie Groups, Birkhäuser, Boston-Basel-Stuttgart, 1981.

[39] _ Irreducible characters of semisimple Lie groups. I, Duke Math. J. 46 (1979), 61-108.

[40] _ Irreducible characters of semisimple Lie groups. II. The Kazhdan-Lusztig conjectures, Duke Math. J. 46 (1979), no. 4, 805-859.

[41] _ Irreducible characters of semisimple Lie groups III. Proof of Kazhdan-Lusztig conjecture in the integral case, Invent. Math. 71 (1983), no. 2, 381-417.

[42] _ Irreducible characters of semisimple Lie groups. IV. Charactermultiplicity duality, Duke Math. J. 49 (1982), no. 4, 943-1073.

[43] - Unitarizability of certain series of representations, Ann. of Math. 120 (1984), 141-187.

[44] _ Branching to a maximal compact subgroup, Harmonic analysis, group representations, automorphic forms, and invariant theory: in honour of Roger E. Howe, Lect. Notes Ser. Inst. Math. Sci. Nat. Univ. Singap., vol. 12, World Sci. Publ., Hackensack, NJ, 2007, pp. 321-401.

[45] Wai Ling Yee, Signatures of invariant Hermitian forms on irreducible highest-weight modules, Duke Math. J. 142 (2008), no. 1, 165-196.

[46] _ Relating signed Kazhdan-Lusztig polynomials and classical Kazhdan-Lusztig polynomials, arXiv:1205.5607.

[47] G. Zuckerman, Tensor products of finite and infinite dimensional representations of semisimple Lie groups, Ann. of Math. (2) 106 (1977), 295-308. 